
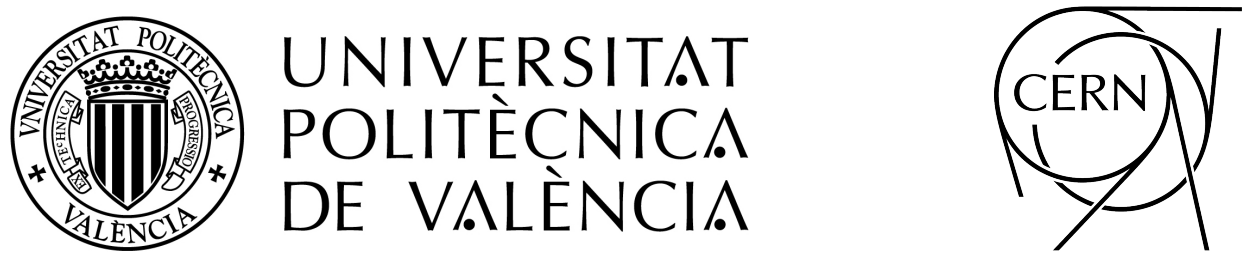

\title{
Comprehensive Study for an Optimized Redesign of the CERN's Antiproton Decelerator Target
}

\author{
Dissertation submitted to obtain the academic degree of \\ $\mathrm{PhD}$ in Industrial Engineering and Production \\ By
}

Claudio Torregrosa Martín

January 2018

Directors:

Dr. José-Luis Muñoz-Cobo

Dr. Marco Calviani 


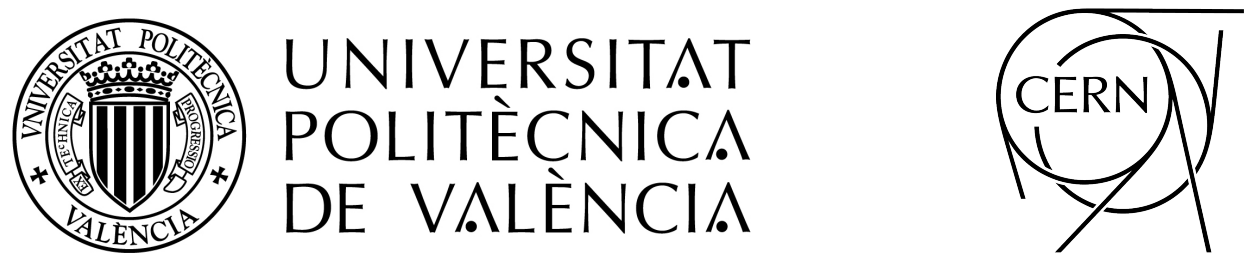

\section{Comprehensive Study for an \\ Optimized Redesign of the CERN's Antiproton Decelerator Target}

Dissertation submitted to obtain the academic degree of

$\mathrm{PhD}$ in Industrial Engineering and Production

By

Claudio Torregrosa Martín

January 2018

CERN's Supervisor:

Dr. Antonio Perillo-Marcone 



\section{Abstract}

The Antiproton Decelerator Target (AD-Target) is a unique device responsible for the production of antiprotons at the European Organization for Nuclear Research (CERN). During operation, intense $26 \mathrm{GeV}$ energy proton beams are impacted into its core, made of a $3 \mathrm{~mm}$ diameter rod of a high density material such as iridium, creating secondary particles -including antiprotons- from the nuclear reactions induced in its interior. This thesis delves into the characteristics of antiproton production and in particular in the mechanical response of the target core material, which is exposed to a rise of temperature of $\sim 2000{ }^{\circ} \mathrm{C}$ in less than $0.5 \mu \mathrm{s}$ each time is impacted by the primary proton beam. A coupled numericalexperimental approach has been applied for this purpose.

Specific computational tools, called hydrocodes, have been used for simulating the extreme dynamic response taking place in the target core and its containing graphite matrix, indicating their potential damage and fragmentation as a result of a high frequency radial compressive-to-tensile pressure wave generated after each proton beam impact.

A challenging first-of-its-kind experiment called HRMT27 was carried out. Several rods of high density materials, candidate for a future optimized target design, such as Ir, W, W-La, Mo, TZM and Ta were brought to equivalent dynamic conditions as reached in the AD-Target core by impacting them with $440 \mathrm{GeV}$ proton beams using the HiRadMat facility. Online instrumentation was used to measure the predicted radial wave, confirming the accuracy of the hydrocode simulations. All of the irradiated target materials except Ta showed internal cracking from conditions 5-7 times below the present in the AD-Target while the latter apparently survived.

Lessons learned are applied for proposing a new optimized target design, including a pressurized-air cooling system, Ta core configuration, and a containing matrix made of expanded graphite (EG). Computational Fluid Dynamic and Structural Finite Element analyses have been carried out to validate the new cooling system and fatigue life of the target assembly. A first prototype of the target core and its containing EG matrix has been built. These activities lead the way into manufacturing a new set of antiproton targets to guarantee antiproton physics at CERN during next decades of operation. 



\section{Resumen}

El Antiproton Decelerator Target (AD-Target) es un dispositivo único responsable de la generación de Antiprotones en la Organización Europea para la Investigación Nuclear (CERN). En operación, intensos haces de protones con una energía de $26 \mathrm{GeV}$ son impactados en su núcleo, un cilindro de $3 \mathrm{~mm}$ de diámetro constituido por un material de alta densidad como el iridio, creando partículas secundarias -entre ellas, antiprotones- como consecuencia de las reacciones nucleares inducidas en el interior de éste. La tesis profundiza en las características del target de producción de antiprotones, y en particular, en la respuesta mecánica de su núcleo, el cual está sometido a un incremento de temperatura de $\sim 2000{ }^{\circ} \mathrm{C}$ en menos de $0.5 \mu s$ cada vez que es impactado por el haz de protones primario. Para ello, una metodología combinando técnicas numéricas y experimentales ha sido llevada a cabo.

Se han aplicado herramientas computacionales específicas, llamadas hydrocodes, para simular la respuesta dinámica originada en el núcleo del target y su matriz contenedora, hecha de grafito, indicando su potencial fragmentación como resultado de una onda radial de alta frecuencia de presión compresión-tracción generada después de cada impacto del haz de protones.

Asimismo, se ha llevado a cabo un experimento llamado HiRadMat27. En éste, varios cilindros de materiales de alta densidad, candidatos para un futuro diseño del target, tales como Ir, W, W-La, Mo, TZM y Ta, han sido expuestos a condiciones dinámicas equivalentes a las alcanzadas en el AD-Target mediante impactos de haces de protones de $440 \mathrm{GeV}$ en la instalación HiRadMat. Se ha usado instrumentación en línea para medir la onda radial pronosticada, confirmando la precisión de las simulaciones de hydrocodes. Todos los materiales irradiados excepto Ta sufrieron agrietamientos internos desde condiciones 5-7 veces menores a las presentes en el $\mathrm{AD}$-Target, mientras que este último aparentemente sobrevivió.

La información obtenida ha sido aplicada para proponer un nuevo diseño optimizado del target, el cual incluye un sistema de refrigeración de aire a presión, una nueva configuración en Ta de su núcleo, y una matriz contenedora hecha de grafito expandido (GE). Se han llevado a cabo cálculos de dinámica de fluidos computacional y elementos finitos para validar el sistema de refrigeración y la vida a fatiga del ensamblaje del target. Además, se ha construido un primer prototipo del núcleo del target y su matrix contenedora. Estas actividades marcan la senda para la fabricación de un nuevo lote de targets que garanticen la física de antiprotones en el CERN durante las siguientes décadas de operación. 



\section{Resum}

L'Antiproton Decelerator Target (AD-Target) és un dispositiu únic responsable de la generació d'Antiprotons a la Organització Europea per la Recerca Nuclear (CERN). En operació, intensos feixos de protons amb una energia de $26 \mathrm{GeV}$ impacten contra el seu nucli, un cilindre de $3 \mathrm{~mm}$ de diámetre constituit per un material de densitat alta com l'iridi, creant partícules secundáries - entre elles, antiprotons - com a conseqüència de les reaccions nuclears induïdes a l'interior d'aquest. La tesis profunditza en les característiques del target de producció d'antiprotons i, en particular, a la resposta mecánica del seu nucli, el qual és sotmès a un increment de temperatura de $\sim 2000{ }^{\circ} \mathrm{C}$ en menys de $0.5 \mu s$ cada vegada que és impactat pel feix de protons primari. Per aixó, s'ha portat a terme una metodologia que combina tècniques numèriques i experimentals.

S'han utilitzat eines computacionals específiques, anomenades hydrocodes, per simular la resposta dinàmica originada al nucli del target $\mathrm{i}$ a la seva matriu contenidora, feta de grafit. La dita resposta, indica la seva potencial fragmentació com a resultat d'una ona radial d'alta freqüència de pressió compressió-tracció generada després de cada impact del feix de protons.

Així mateix, s'ha portat a terme un experiment anomenat HiRadMat27. En aquest, varis cilindres de materials d'alta densitat, candidats per un futur diseny del target, tals com Ir, W, W-La, Mo, TZM i Ta, han estat exposats a condicions dinàmiques equivalents a les assolides a l'AD-Target mitjanant impactes de feixos de protons de $440 \mathrm{GeV}$ a l'instalació HiRadMat. S'ha utilitzat instrumentació en línia per mesurar l'ona radial pronosticada, confirmant la precisió de les simulacions d'hydrocodes. Tots el materials irradiats excepte Ta van sofrir esquerdaments interns desde condicions de 5-7 vegades menors a les presents a l'AD-Target, mentres que aquest últim aparentment va sobreviure.

L'informació obtinguda ha estat aplicada per proposar un nou diseny optimizat del target, el qual inclou un sistema de refrigeració de l'aire a pressió, una nova configuració en Ta del seu nucli, i una matriu contenidora feta de grafit expandit (GE). S'han portat a terme càlculs de dinàmica de fluids computacionals i elements finits per validar el sistema de refrigeració i la vida a fatiga de l'ensambladura del target. S'ha construit un primer prototip del nucli del target i la seva matriu contenidora. Totes aquestes activitats marquen la sendera per a la fabricació del nou lot de targets que garantitzin la física d'antiprotons al CERN durant les següents décades d'operació. 



\section{Acknowledgments}

First of all, I would like to express my deep gratitude to Dr. Marco Calviani and Dr. Antonio Perillo-Marcone for trusting me and giving me the chance of carrying out my PhD at CERN and, in particular, in this challenging and exciting topic. Their supervision and dedication have been fundamental for the achievement of this thesis, thanks to the ideal tandem that they form; Antonio from the point of view of mechanical engineering, with his natural intuition, concise and smart advices. And Marco with his enthusiasm, incredible knowledge of physics and accelerator technology, and his keen involvement for triggering projects and new paths of research.

My sincere thanks to Prof. José Luis Muñoz-Cobo for being my director and his kind supervision even from the distance, with his dedication and interest in my progresses, the inestimable advices supported by his outstanding research experience, and the thorough revision of this manuscript.

There are also many people who have contributed and have been fundamental in the achievement of this thesis, in particular in the design, assembling and execution of such a complex and unique experiment as HRMT27-RodTarg. So many that unfortunately I cannot even mention all of them. Nevertheless, I do not want to forget Dr. Mark Butcher for his expertise and involvement in the electronic part of the experiment, including instrumentation setup. The high quality data recoded and presented here would not exist without him. To Luca Gentini, for his sharpness and dedication in the challenging design of the experiment. To Julien Broussart, Damien Grenier and all the people of the EN-STI workshop, for their professionalism and support during the manufacturing of the experiment. To Philippe Frichot, from the CERN main workshop, for his dedication for searching and finding companies brave enough to machine and polish our hard refractory metals up to unprecedented precisions. To Stephane Burger and Thibaut Lefevre for the development and implementation of the beam position monitors, which were fundamental for the experiment. To Aymeric Bouvard and Adrian Fabich, for the installation and coordination of the experiment. To Maxime Bergeret for his support in the huge amount of Autodyn simulations necessary for setting up 
the instrumentation prior to experiment, to Nicola Solieri for his support in the post-experiment simulations of the excited bending modes, as well as to Elvis Fornasiere for his involvement in the dissembling of the irradiated targets. I wish him the best luck for his $\mathrm{PhD}$ covering the PIEs of the irradiated targets.

I also want to thank to David Horvath, for providing with all the FLUKA energy depositions used in this thesis. To my colleague and friend Edmundo Lopez for his support in the CFD and structural calculations of the new target design presented in the last chapter, as well as to Edouard Grenier-Boley, Romain Ferriere, Mickael Crouvizier and Laurent Prever-Loiri for their respective involvements in the manufacturing of the first target prototype. I do not want to forget either Prof. Lorenzo Peroni and Dr. Martina Scapin from Politecnico di Torino for their accessibility and will for starting a collaboration with us, and the dynamic characterization of tungsten and iridium.

In addition, I do not want to miss the opportunity to mention my dear colleagues and friends of the TCD section at CERN, to the ones that are still here and the ones that have left. Thanks for this fantastic working and after-working atmosphere that we shared over these years in Geneva. To Iñigo, Filippo, Dillon, Anubhav, Marcin, Tobias, Mario, Stefano, Giulia, David and Aurelio. To Estrella for her invaluable friendship and support over these years (and the translation of the abstract!). To Fernando, and my amazing flatmates-family Raffaele and Maria.

I want also to mention my friends from Sweden and around the world, to Barri for the great time we had there, and to Deniz for still believing that I will eventually destroy the world. To Vero, who I miss so much, for being always there no matter the distance, for our short but always intense getaways, and for maintaining alive the hope of being closer in the future.

I do not forget also all my friends in Spain, who I also miss a lot. To Carmen because she always believed in me, to Josep and Sergi and the people from Valencia, with who I wish I could share city again at some point, and of course to my friends of Alicante, to Jorge, Monxo, Giner, Xente and Zafra. I feel proud just to think that you are proud of me.

Finalmente, agradecer infinitamente a mi familia, primos y tíos. Y más que a nadie a mis padres y hermana, por su cariño y apoyo incondicional, ahora y siempre. Este doctorado es tan suyo como mío. 


\section{Contents}

Abstract iii

Resumen $\quad$ v

Resum vii

Acknowledgments $\quad$ ix

Contents $\quad$ xi

Acronyms and Nomenclature xvii

1 Introduction 1

1.1 Motivation and Objectives of the Thesis. . . . . . . . . . . 1

1.2 Antiproton Production at CERN $\ldots \ldots \ldots \ldots \ldots \ldots \ldots \ldots$

1.2.1 Physics of Antiproton Production . . . . . . . . . . . . 5

1.2.2 Previous Designs of Antiproton Targets at CERN . . . . . . . . . . . . 6

1.2 .3 Current Target Design . . . . . . . . . . . . . . . . . . . . . 9

1.3 Main Inherent Concerns and Operational Experience of the CERN's An-

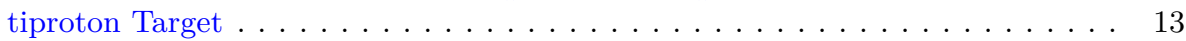

1.3.1 Sudden Rise of Temperature and Stress Waves in the Target Core. . . . . . 13

1.3.2 Radiation Damage . . . . . . . . . . . . . . . . . 15

1.3.3 Concerns on the Target Envelope . . . . . . . . . . . . . . . . . 16 
1.4 Comparison with other High Power Target Facilities. . . . . . . . . . . . 17

1.4.1 The Pbar Source at Fermilab. . . . . . . . . . . . . . . . . . . 18

1.5 Candidate Core Materials for the Future AD-Target Design. . . . . . . . . . 20

1.5 .1 Iridium . . . . . . . . . . . . . . . . . . . . 20

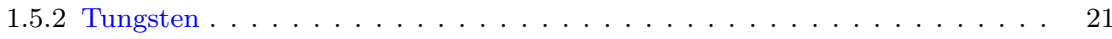

1.5.3 Tungsten doped with Lanthanum Oxide . . . . . . . . . . . . . . . . . 24

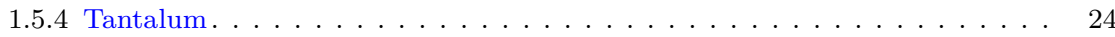

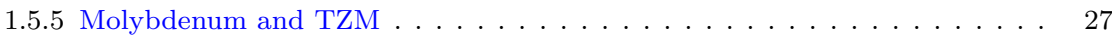

2 Computational Methods for Simulating the Response of the AD-Target Core 29

2.1 Introduction . . . . . . . . . . . . . . . . . . . . . . . . . . . 29

2.2 Principles of Stress Wave Propagation in Solids and Vibration Theory . . . 31

2.2.1 Stress Wave Propagation in Solids . . . . . . . . . . . . . . . . 31

2.2 .2 Free Vibrations Modes of a Cylinder . . . . . . . . . . . . . . . . . . 39

2.3 Energy Deposition in the AD-Target: FLUKA Monte-Carlo Code. . . . . . . 44

2.4 Thermo-Mechanical Simulations using Implicit-FE ANSYS Mechanical . . . 46

2.5 Limitations of Implicit-FE ANSYS® Mechanical for Simulating the AD-

Target Dynamic Response . . . . . . . . . . . . . . . . . . . . . . . . . 48

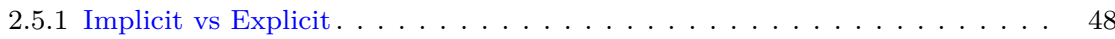

2.5.2 Strain Rate Dependence of the Material Response. . . . . . . . . . . . . . . 49

2.5.3 Non-linearity, Yielding and Fracture . . . . . . . . . . . . . . . 50

2.6 Introduction to Hydrocodes . . . . . . . . . . . . . . . . . . . . 51

2.6.1 Stress Tensor Decomposition . . . . . . . . . . . . . . . . . 53

2.6.2 EOS - Governing the Hydrostatic Component . . . . . . . . . . . . . 55

2.6.3 Strength Model - Governing the Deviatoric Component . . . . . . . . . . . 59

2.6.4 Failure Models . . . . . . . . . . . . . . . . . . . . . . 63

2.7 Computational Method for the Resolution of Dynamic Response of the

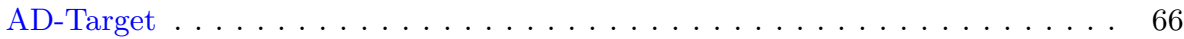

2.7 .1 Computational Approach . . . . . . . . . . . . . . . . . 66

2.7.2 Geometry and Computational Domain. . . . . . . . . . . . . . . 67

2.7.3 Employed EOS, Strength and Failure Models. . . . . . . . . . . . . . . 69

2.8 Configurations and Objectives of the Performed Simulations . . . . . . . . . 74

2.8.1 From Simple Assumptions to Complex Models . . . . . . . . . . . . . . 74

2.8.2 Parametric Analysis of Influence of Proton Pulse Length on the Pressure

Response. . . . . . . . . . . . . . . . . . . . . . . . 74 
2.8.3 Parametric Analysis of Influence of Core Cladding on the Pressure Re-

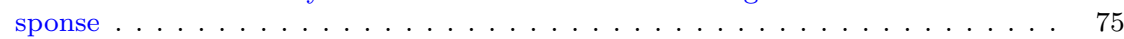

2.8.4 Comparisons of Iridium and Tungsten as Core Material . . . . . . . . . . 76

2.8.5 Analysis of Dynamic Response of the Graphite Matrix . . . . . . . . . . . . 76

\section{Results of the Dynamic Response of the Target Core under} Proton Beam Impact

3.1 Adiabatic Increase of Temperature in the Target . . . . . . . . . . . . . 78

3.2 Elastic Response of the Target Core . . . . . . . . . . . . . . . . . . . . 79

3.3 Plastic Response of the Target Core . . . . . . . . . . . . . . . . . . . . . 82

3.4 Importance of the Proton Pulse Length on the Pressure Response . . . . . . 84

3.5 Use of Core Cladding as a Strategy to Reduce Tensile Pressure in the Core 86

3.6 Application of Failure Models to the Cases of Study . . . . . . . . . . . . . . 88

3.7 Comparison between Tungsten and Iridium as Core Material . . . . . . . . . . 89

3.8 Response of the Graphite Matrix. . . . . . . . . . . . . . . . . . . . . . . . 90

3.9 Main Conclusions and Discussion of the Results . . . . . . . . . . . . . 93

\section{The HRMT27 Experiment: Recreating Equivalent Conditions} as in the AD-Target $\quad 97$

4.1 Introduction and Motivation of the Experiment . . . . . . . . . . . . 97

4.2 Definition of Goals of the Experiment . . . . . . . . . . . . . . . 100

4.3 Involved Challenges . . . . . . . . . . . . . . . . . . . . . . . 101

4.3.1 Creating Equivalent Conditions in the HiRadMat Targets as in the AD-

Target . . . . . . . . . . . . . . . . . . . . . 101

4.3.2 Challenges in terms of Accuracy and Beam Monitoring. . . . . . . . . . . . 102

4.3 .3 Instrumentation Selection and Configuration . . . . . . . . . . . . . . 102

4.3 .4 Activation of Targets. . . . . . . . . . . . . . . . . . . . . . . . 103

4.4 Design of the Experiment. . . . . . . . . . . . . . . . . . . . . . . . 104

4.4.1 Design of the Targets and Beam Parameters . . . . . . . . . . . . . . . . . 104

4.4 .2 General Layout . . . . . . . . . . . . . . . . . . . . . . . . . 113

4.4 .3 Instrumentation Selection. . . . . . . . . . . . . . . . . . . . . . . . 117

4.4.4 Thermal and Structural Calculations of the Experimental Setup . . . . . . . 121

4.5 List of Investigated Target Materials . . . . . . . . . . . . . . . . . . . . 129

4.5 .1 Iridium . . . . . . . . . . . . . . . . . . . . . 130

4.5.2 Pure Tungsten and Tungsten doped with Lanthanum Oxide . . . . . . . . 131 
4.5 .3 Tantalum. . . . . . . . . . . . . . . . . . . . . . . . . 132

4.5.4 Molybdenum and TZM . . . . . . . . . . . . . . . . . . . 133

4.5.5 Tungsten Cladded in Tantalum . . . . . . . . . . . . . . . . . . . . 133

4.6 Execution of the Experiment $\ldots \ldots \ldots \ldots \ldots \ldots \ldots$

4.6.1 Targets Alignment Procedure . . . . . . . . . . . . . . . . . . 137

4.6.2 Operation Procedure. . . . . . . . . . . . . . . . . . . . . . . . 138

4.6 .3 Pulses Impacted on Targets . . . . . . . . . . . . . . . . . . . . . . 140

4.6.4 Beam Stability during Operation . . . . . . . . . . . . . . . . . . . . . 141

4.6.5 Radiation Levels during Operation . . . . . . . . . . . . . . . . . . . 144

5 The HRMT27 Experiment: Online Results 149

5.1 Thermocouples and Pyrometer Recordings . . . . . . . . . . . . . . . . 151

5.2 Dynamic Response at Low Intensity Pulses. . . . . . . . . . . . . . . . . 153

5.3 Change of the Dynamic Response when Increasing Intensity . . . . . . . . . 161

5.3.1 Targets Experiencing Plastic Deformation and Internal Damage . . . . . . . 161

5.4 High Intensity Pulses. Extensive Target Damage . . . . . . . . . . . . . . 166

5.4 .1 Tungsten at High Intensity . . . . . . . . . . . . . . . . . . . 166

5.4 .2 Massive Fragmentation of the Iridium Target. . . . . . . . . . . . . . 167

5.5 Summary of Response of the Irradiated Targets . . . . . . . . . . . . . 171

5.6 Discussion . . . . . . . . . . . . . . . . . . . . . . . 174

6 The HRMT27 Experiment: First PIEs and Experiment vs Simulation Comparisons $\quad 175$

6.1 Pictures of the Targets after the Experiment . . . . . . . . . . . . 176

6.1 .1 Mo and TZM targets. . . . . . . . . . . . . . . . . . . 176

6.1 .2 Ta targets . . . . . . . . . . . . . . . . 177

$6.1 .3 \mathrm{~W}$ and W-La targets. . . . . . . . . . . . . . . . . . 178

6.1 .4 Ir targets . . . . . . . . . . . . . . . . . . . . . . . . . . . 181

6.1 .5 W-Ta cladded target. . . . . . . . . . . . . . . . . . . . 183

6.2 Graphite Clamps Damage . . . . . . . . . . . . . . . . . . . . 184

6.3 Measurements of Targets Deformation . . . . . . . . . . . . . . 185

6.4 Comparisons between Hydrocode Simulations and Experimental Results . . 189

6.4 .1 Comparisons at low intensity pulses . . . . . . . . . . . . . . . 189

6.4.2 Medium Intensities and Failure Model Benchmarking. . . . . . . . . . . . . 193

6.4.3 Comparisons at High Intensity Pulses for \#8W3 and \#12Ir3 targets . . . . 197 
6.5 Simulations of Targets Bending and Experimental Crosscheck . . . . . . . . 200

6.6 Main Outcomes of the Experiment and Proposed Improvements. . . . . . . 203

7 Conceptual Design of the New AD-Target 207

7.1 Proposed Core Configuration and Containing Matrix . . . . . . . . . . . . . 209

7.1.1 New core material: Tantalum . . . . . . . . . . . . . . . . . . . . . . . . . 209

7.1 .2 New core geometry configuration . . . . . . . . . . . . . . . . . . . 209

7.1.3 Compressed Expanded Graphite (EG) as target core containing matrix. . . 211

7.1.4 Prototyping Activities for Manufacturing an EG-Matrix Embedding the Target Core . . . . . . . . . . . . . . . . . . . . . . . . . . . . 213

7.2 Proposed Pressurized Air Cooling System. . . . . . . . . . . . . . . . 220

7.2 .1 Proposed Geometry . . . . . . . . . . . . . . . . . . . . . 220

7.2.2 CFD Simulations and Cooling Flow Parameters . . . . . . . . . . . . . 221

7.2 .3 Thermal Analysis of the Target Assembly . . . . . . . . . . . . . . . . . . . . 224

7.3 Structural and Fatigue Analysis of the Ti-6Al-4V Beam Windows . . . . . 226

7.3.1 Stresses Reached in the Windows . . . . . . . . . . . . . . . . . . 227

7.3 .2 Fatigue Analysis . . . . . . . . . . . . . . . . . . . . . . . . . . 229

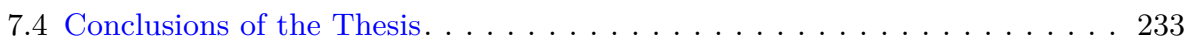

$\begin{array}{ll}\text { Bibliography } & 237\end{array}$ 



\section{List of Acronyms}

AA Antiproton Accumulator

AC or ACOL Antiproton Collector

AD Antiproton Decelerator

bcc body-centered cubic

BDF Beam Dump Facility

BID Beam Intercepting Device

BIF Brittle intergranular fracture

BNL Brookhaven National Laboratory

BTF Brittle transgranular fracture

CCC CERN Control Center

CMM Coordinate measuring machine

CNGS CERN Neutrinos to Gran Sasso

DPA Displacement per atom

EDM Electro-discharge Machining

ELENA Extra Low ENergy Antiproton Ring

ESS European Spallation Source 
fcc face-centered cubic

FE Finite Element

HIP Hot Isostatic Pressing

HRMT HiRadMat (High Radiation to Materials)

HTC Heat transfer coefficient

ISOLDE On-line isotope mass separator

J-C Johnson-Cook

LANL Los Alamos National Laboratory

LDV Laser Doppler Vibrometer

LEAR Low Energy Antiproton Ring

LLNL Lawrence Livermore National Laboratory

MPDB Material Properties Database

MTS Mechanical Threshold Stress

nTOF Neutron Time of Flight

ORNL Oak Ridge National Laboratory

Pbar Antiproton

PIE Post Irradiation Examination

POT Protons on Target

ppp Protons per pulse

PS Proton Synchrotron

PTW Preston-Tonks-Wallace 
RAL Rutherford Appleton Laboratory

S-G Steinberg-Guinan

SHPB Split Hopkinson Pressure Bar

SNS Spallation Neutron Source at Oak Ridge National Laboratory

SPS Super Proton Synchrotron

VM Von Mises

Z-A Zerilli-Amstrong

\section{Nomenclature}

$[C] \quad$ Damping matrix

$[K] \quad$ Stiffness matrix

$[M] \quad$ Mass matrix

$\alpha \quad$ Linear thermal expansion coefficient

$\bar{p} \quad$ Antiproton

$\ddot{u} \quad$ Acceleration vector

$\dot{\epsilon}_{i j} \quad$ ij Component of the strain rate tensor

$\dot{u} \quad$ Velocity vector

$\epsilon_{f} \quad$ Failure strain

$\epsilon_{i j} \quad$ ij Component of the strain tensor

$\epsilon_{v} / \epsilon_{e q}$ Volumetric strain / Equivalent strain

$\gamma \quad$ Grüneisen parameter 
$\lambda_{\bar{p}} \quad$ Antiproton reabsorption length

$\lambda_{p} \quad$ Proton inelastic scattering length

$\mu \quad$ Volumetric parameter $\mu=\rho / \rho_{0}-1$

$\nu \quad$ Poisson's ratio

$\rho \quad$ Density

$\sigma_{1} / \sigma_{2} / \sigma_{3}$ Principal stresses

$\sigma_{a} \quad$ Amplitude of the alternating stress of a fatigue load

$\sigma_{i j} \quad$ ij Component of the stress tensor

$\sigma_{m} \quad$ Mean stress of a fatigue load

$\sigma_{u} \quad$ Ultimate tensile strength

$\sigma_{V M} \quad$ Von Mises equivalent stress

$\tau_{f} \quad$ Failure shear stress

c Speed of light in vacuum (at particle physics context)

$C_{v} \quad$ Specific heat capacity at constant volume

$C_{0}$ or $c$ Elastic speed of sound in the bulk (at shock physics context)

$C_{p} \quad$ Velocity of propagation of a plastic wave

$e / \dot{e} \quad$ Specific Energy / Partial derivative of specific energy with respect to time

$F \quad$ Force vector

$f_{x} / f_{y} / f_{z}$ Body forces in $\mathrm{x}, \mathrm{y}, \mathrm{z}$

$K \quad$ Bulk Modulus

$m \quad$ mass 
$P \quad$ Pressure

$p \quad$ Proton

$R \quad$ Stress ratio $\sigma_{\max } / \sigma_{\min }$ of an alternating load in fatigue context

$s_{i j} \quad$ ij Component of the deviatoric stress tensor

$t \quad$ time

$T / T_{r} / T_{m}$ Temperature / Reference Temperature / Melting Temperature

$u \quad$ Displacement vector

$U_{s} \quad$ Shock front velocity

$U_{p} \quad$ Particle velocity (shock physics context)

$U_{s} \quad$ Shock front velocity

V Volume

$v \quad$ Specific volume

$x / y / z$ Displacement in $\mathrm{x}, \mathrm{y}, \mathrm{z}$

$Y_{0} \quad$ Yield strength

Z Atomic number

$h \quad$ Convective heat transfer coefficient 



\section{Chapter 1}

\section{Introduction}

\subsection{Motivation and Objectives of the Thesis}

In accelerator facilities, intense and high energy proton beams are commonly impacted onto solid fixed materials (targets) in order to produce new particles and secondary beams through the interaction of the proton beam with the atoms and nuclei of these fixed materials. The geometrical design and material selection of these targets is completely specified by the kind of particles and secondary beam desired.

A very specific case of target is the Antiproton Decelerator target (AD-Target) at the European Organization for the Nuclear Research (CERN) in Geneva, Switzerland. The aim of the AD-Target system is to produce antiprotons for the Antiproton Decelerator facility. Antiprotons are produced by colliding a proton beam of $26 \mathrm{GeV} / \mathrm{c}$ from CERN Proton Synchrotron (PS) with a fixed target made of a dense and high- $Z$ material. The high energy collision of the proton beam with the nuclei of the target creates a shower of secondary particles, and among them, antiprotons. The generated antiprotons travel through the target assembly and are collected downstream by a magnetic focusing device (magnetic horn) using their charge. After this, antiprotons of $3.5 \mathrm{GeV} / \mathrm{c}$ are selected and magnetically transferred via the injection line to the Antiproton Decelerator (AD) Complex, where they are slowed down to a momentum of $100 \mathrm{MeV} / \mathrm{c}$ by combined electron cooling and stochastic cooling [1]. The aim of slowing down the antiprotons is to finally confine them by a magnetic trap and subsequent production of, among others, anti-hydrogen for antimatter research experiments.

Antiparticles should be identical to matter particles except for the sign of their electric charge. It is well known, however, that matter and antimatter are not exact 


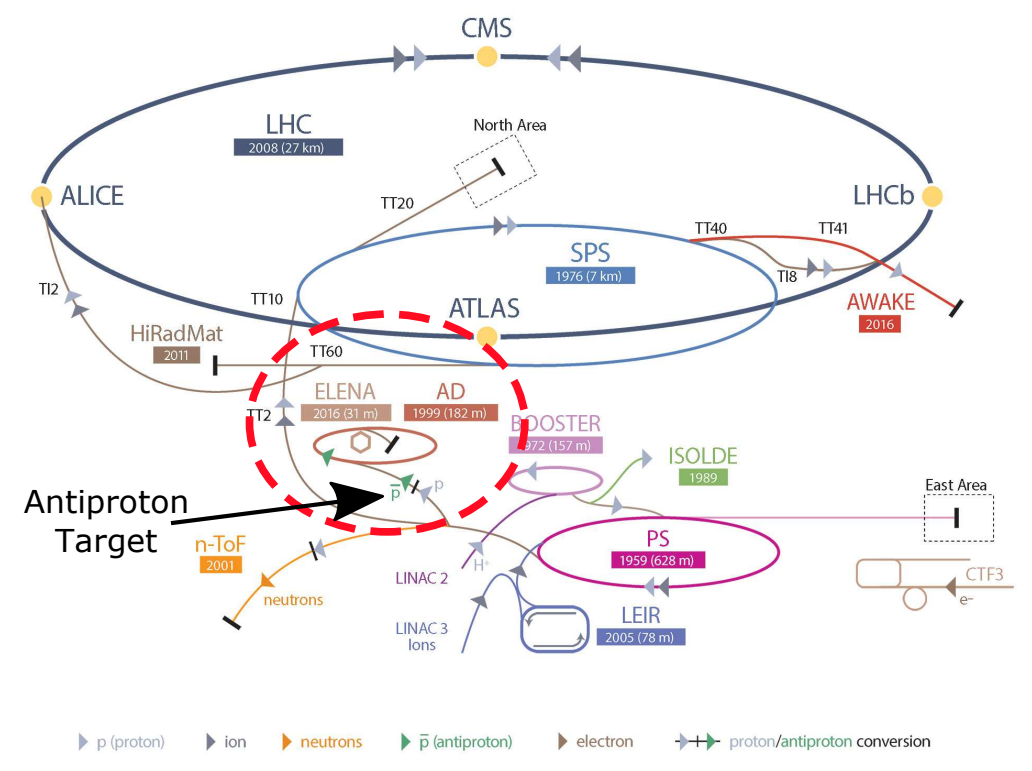

Figure 1.1: CERN Accelerator Complex. In the schematics the AD and future ELENA complex are highlighted. The schematics includes as well a representation of the antiproton target, receiving protons from the PS (Proton Synchrotron) and "converting" them in antiprotons, which are injected to the AD.

opposites; nature seems to have a one-part in 10 billion preference for matter over antimatter. Understanding matter-antimatter asymmetry is one of the greatest challenges in physics today. Several experiments are devoted to this purpose using the antiprotons created in the AD-Target and decelerated in the AD ring. Some of them are ATRAP [2], ASACUSA [3], ATHENA [4], ALPHA [5], or AEGIS [6]. Figure 1.1 shows a schematics of the CERN Accelerator Complex and highlights the $\mathrm{AD}$ facility and the representation of the antiproton target, receiving proton beams from the PS and injecting antiprotons to the AD.

In order to continue with this search for new anti-matter physics for the future 20 years, a new machine called ELENA (Extra Low ENergy ring) is currently under construction at CERN. This machine provides an extra cooling ring, receiving antiprotons from the $\mathrm{AD}$ and decreasing further its momentum down to $13.7 \mathrm{MeV} / \mathrm{c}$ (which corresponds to a kinetic energy of $100 \mathrm{keV}$ ). In this way, this machine will avoid a step currently necessary for capturing the antiprotons which consists in passing them through a thin foil. This step has the highly undesired effect of annihilating more than $99 \%$ of them. The new AD/ELENA complex will therefore increase the efficiency of antiproton production by several orders of magnitude [7]. 


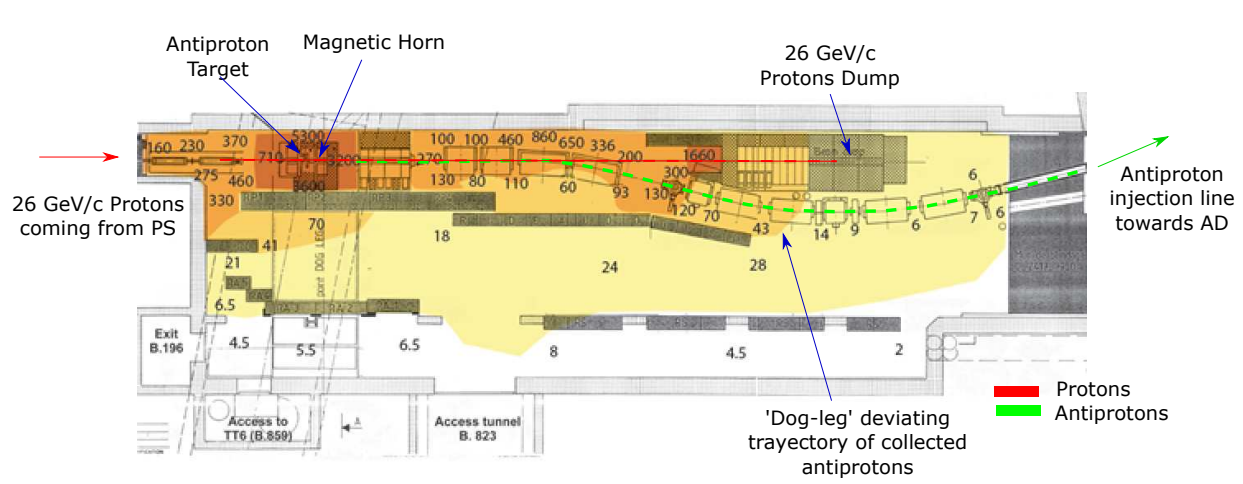

Figure 1.2: Schematics of the AD-Target Area, showing the injection line from the PS, the target, the magnetic horn, the "dog-leg" and the injection line towards AD.

In the context of construction of ELENA, an extensive project of consolidation activities in the AD-Target Area is currently taking place in order to guarantee a reliable supply of antiprotons to the AD/ELENA complex during future operation. This area (shown in figure 1.2) consists of an underground hall to house the equipment necessary for supplying antiprotons to the AD. Antiproton science started in CERN in the late 70's and, despite several changes and upgrades in the antiproton storage rings during the 80 's and 90 's, this area has remained practically unchanged from the late 80's [8]. An upgrade is therefore planned to take place during the CERN Long Shutdown 2 (2019-2020) and will involve a general refurbishment, including decontamination, re-cabling and replacement of equipment. In addition, most critical components of the area will be subjected to a major upgrade, namely (i) antiproton production target (which main concept has been unchanged since 1987 [9]), (ii) $400 \mathrm{kA}$ pulsed magnetic horn and (iii) target and horn's trolleys. The goals of this upgrade is to address specific requirements of the future operation, to investigate new designs and operational procedures to improve the current antiproton production yield [10], and to increase the readability of the area.

Among the three introduced systems which will be upgraded, the present thesis is devoted to the target. The relevance of the antiproton target is not only motivated by the significance of its purpose -to produce new particles to serve as a window to new physics- but for the challenge that involves to overcome the engineering problem of its design. The characteristics of antiproton production require a very compact target in order to avoid antiproton re-absorption in the surrounding material and to be as close as possible to a punctual source for the antiproton collector system placed downstream [11, 12] (currently a magnetic horn). For this reason, a very thin rod of a high density material and a very focused primary proton beam have to be used. This results in extremely high energy depositions reached inside the target core as a consequence of each proton beam impact, which makes the 
antiproton target a unique case even among high energy particle-producing primary targets. The current target design was partially constructed by an iterative process of improvements due to the complexity of physical phenomena occurring inside the target material under the effect of the proton beam impact.

The goal of the present thesis is to carry out a comprehensive analysis of the characteristics of the CERN's antiproton target in order to propose an optimized new design which can provide a robust operation during the next decades of antiproton physics at CERN. This study consists mainly in four steps that are summarized below:

I A deep analysis of the existing target and literature review of the present and past designs is carried out and presented in Chapter 1. This study was fundamental in order to understand peculiarities of the antiproton production target and identify the main operational concerns that limited the target life in the past. In addition, a literature review and detailed description of candidate materials for the AD-Target core is presented.

II According to the literature review two main operational concerns have been identified; (i) mechanical damage in the core of the target material due to thermal shock and high level of dynamic stresses and (ii) long term radiationinduced damage in the material. This thesis is mainly focused in the former since, as it will be shown, radiation induced damage is expected to take place in the longer term, when the target material has suffered already an important level of damage due to thermal shock. Advanced and highly specific computational tools, hydrocodes, are applied to solve the thermally-induced dynamic loading reached in the core of the antiproton target. The results of this numerical analysis allow to identify the governing phenomena which leads to the target material damage. These analyses are presented in Chapters 2 and 3.

III Additionally to the numerical simulations, it will be shown that experimental tests are necessary in order to complete the complex simulations performed, validate them, and gain experimental insights on materials' response. With this purpose, an experiment called HRMT27-RodTarg- was performed using the HiRadMat facility at CERN [13]. In this experiment, the extreme conditions identified in Chapters 2 and 3 were recreated in different high density materials by exposing them to direct impacts of intense proton beams from the SPS (Super Proton Synchrotron). This experiment is used as well to assess the material selection for the future target design. Chapters 4, 5 and 6 of this thesis are respectively dedicated to the description, results, numericalexperimental comparisons and first Post Irradiation Examinations (PIEs) of the experiment. 
IV Finally, in Chapter 7 the lessons learned during this research are applied to a new target design proposal. This chapter includes as well simulations of a new proposed pressurized air cooling system of the target, which as it will be described, will be more convenient for future operation of the target area.

\subsection{Antiproton Production at CERN}

As a first step of the re-design procedure, a comprehensive study of the current and past designs is necessary in order to fully understand the antiproton production requirements and identify the main operational concerns. The current target design was partially constructed by an iterative process of improvements due to the complexity of physical phenomena occurring inside the target material under the effect of the proton beam impact. This iterative process was extended for more than 10 years and for this reason there is not an integral design report of the target itself but a spread of single publications documenting particular issues and concerns. This fact, coupled with the circumstance that the design was carried out almost 30 years ago with poor comprehensive design reports, makes the follow-up of the target design characteristics not a straightforward process.

Antiproton science started in CERN in the late 70's, with the Antiproton Accumulator (AA) and Low Energy Antiproton Ring (LEAR). These facilities were providing antiprotons for the proton-antiproton collisions in the Super Proton Synchrotron (SPS) for the $\mathrm{Sp} \bar{p} \mathrm{~S}$ collider program which was in operation until 1984. In 1986 the Antiproton Collector (ACOL or AC) was built around the AA in order to increase the capability for storing antiprotons. The LEAR continued taking antiproton beams from the AC/AA until the end of 1996. In 1997, the AA was dismantled and the $\mathrm{AC}$ converted into the current $\mathrm{AD}$, which provided low energy antiprotons in a simpler way $[14,15]$. The AD has been operating since then and with the ELENA upgrade its operation is guaranteed for the next decades.

\subsubsection{Physics of Antiproton Production}

Antiprotons are mainly produced by collision of the protons coming from a high energy beam with the nucleus of the target material according to the reaction of eq. 1.1, where $\mathrm{p}$ is the incident proton, $\mathrm{N}$ is a nucleus of the target material, $\bar{p}$ is the antiproton produced and $N^{*}$ and $X$ are the remaining excited nucleus and all other particles produced in the collision. This process requires a minimum proton kinetic energy in the laboratory system above the $\bar{p}$ threshold of $5.6 \mathrm{GeV} / \mathrm{c}[16$, $10]$.

$$
p+N \rightarrow N^{*}+\bar{p}+p+X
$$


The produced antiprotons in the reaction are scattered following an isotropic angular distribution in the center of mass (c.m.) system of the two interacting nucleons [11]. This isotropy in the c.m. system leads to a relatively wide angular distribution in the laboratory system -in the range of 50-100 mrad- with respect to the beam direction [17]. These created antiprotons have also a wide range of energy. Nevertheless, the downstream magnetic horn is optimized for collecting the emitted $\bar{p}$ which have a momentum centered in $\sim 3.6 \mathrm{GeV} / \mathrm{c}$ with a momentum spread of $\Delta p / p \sim 6 \%$ [18]. Given the wide angular distribution of the emitted antiprotons in the laboratory system, the target must be made as thin as possible in the radial direction so that re-absorption probability in the sideway target material is small. For this reason, a thin rod (about 2-3 $\mathrm{mm}$ diameter) of a high density material is currently used as target core.

The materials considered for the first designs were copper, tungsten, rhenium and iridium $[19,20]$. The length of the target rod must be long enough so that most of the incident protons interact with the target material and short enough so that most of the produced antiprotons leave the target material without being reabsorbed. This length was found to be roughly 1-1.5 times the proton nuclear inelastic scattering length $\lambda_{p}$ [11]. The choice of a high density material is motivated by the need to make the target as similar as possible to a punctual source, which enhances the antiproton collection by the downstream focusing system, and by maintaining a length amongst 1-1.5 $\lambda_{p}$, which therefore guarantees on the average at least one nucleus interaction [21]. For the same reason, the spot size of the proton beam should be as small as possible (strongly focused primary beam). This fact leads to an extremely high deposited energy density in the target material for each proton pulse, which brings several concerns and limitations for the target life as it will be seen in the next sections.

\subsubsection{Previous Designs of Antiproton Targets at CERN}

The first target design used for AA dates from 1979 (shown in figure 1.3). This design consisted of a forced air external cooled target. The core of the target was made by 11 tungsten rods of $3 \mathrm{~mm}$ diameter, and $10 \mathrm{~mm}$ length each. The aim of using several short rods of reduced length it was to minimize the dynamic stress produced by a longitudinal wave induced by thermal shock, which was identified by analytical calculations made by P. Sievers [22]. The target rods were embedded inside a graphite tube of $30 \mathrm{~mm}$ outer diameter. The objective of the graphite container was to serve as confining matrix for the target core. Graphite was selected because of its low density, which reduces the reabsorption of antiprotons while they are emitted from the target core, resistance to elevated temperatures, high enough thermal conductivity to dissipate the heat from the target rods [17] and easy manufacturing. The tungsten-graphite assembly was inserted in an aluminum envelope with several external fins as shown in figure 1.3(a). 


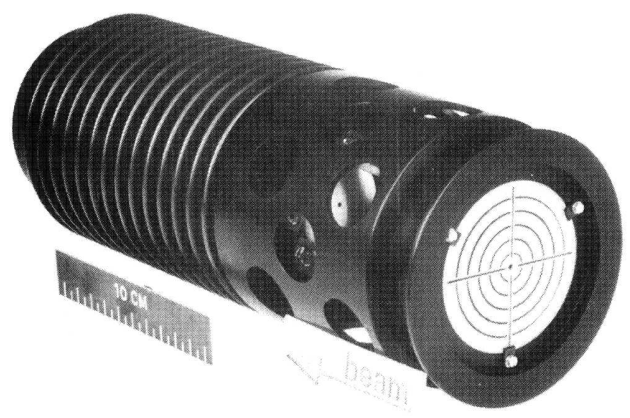

(a)

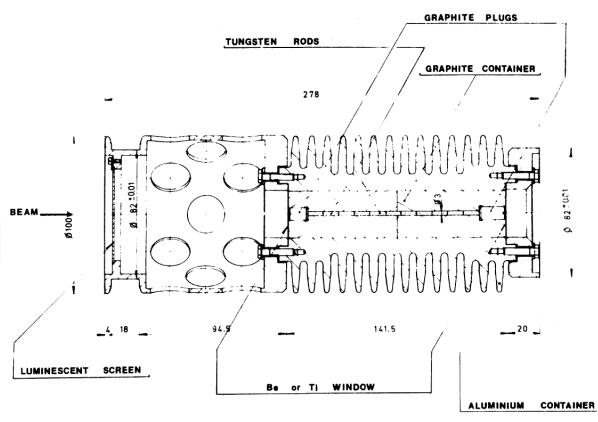

(b)

Figure 1.3: Picture and schematics of the first antiproton target used for AA in 1979 [12].

This target was designed for an incident proton $26 \mathrm{GeV} / \mathrm{c}$ beam of $10^{13} \mathrm{ppp}$ (protons-per-pulse), with beam diameter of $3 \mathrm{~mm}$ and proton burst duration of $0.4 \mu \mathrm{s}$. The repetition rate (period of time between two pulses) was $2.4 \mathrm{~s}$ [12].

During the first six years of operation, from 1981 to 1987, an active research program was performed in order to assess the different concerns which limited the operation life of the target as well as to increase the antiproton production yield. Several target rod materials were tested and used, including copper, rhenium, tungsten and iridium. All designs were based on the air cooled concept presented above. Iridium and rhenium were considered as they are amongst the highest density known elements, with densities of 22.56 and $21.1 \mathrm{gr} / \mathrm{cm}^{3}$ respectively. Despite its lower density, copper presented other advantages such as lower operational temperatures and more ductility.

It is worth noting that for the copper design, a longer target rod, in the range of $11 \mathrm{~cm}$, was necessary due to its higher inelastic length compared to Ir, W or Re. The target envelope was designed in such a way that different target lengths could be tested without changing the whole design. A discussion and comparison of the performance and concerns of the different materials used is provided in the next section.

During these years of research, special attention was given to the reduction of the target length. As already mentioned, shorter target length increases the phasespace density of low angle scattered antiprotons, which enhanced its collection in the downstream focusing system. In particular with the one used at that time consisting in Lithium lens [23]. Figure 1.4 shows one of the lay-out used during 1985. In this design, a rhenium target of $50 \mathrm{~mm}$ long was used [24], almost half of the length compared to the first designs. The iridium rods were embedded in a 


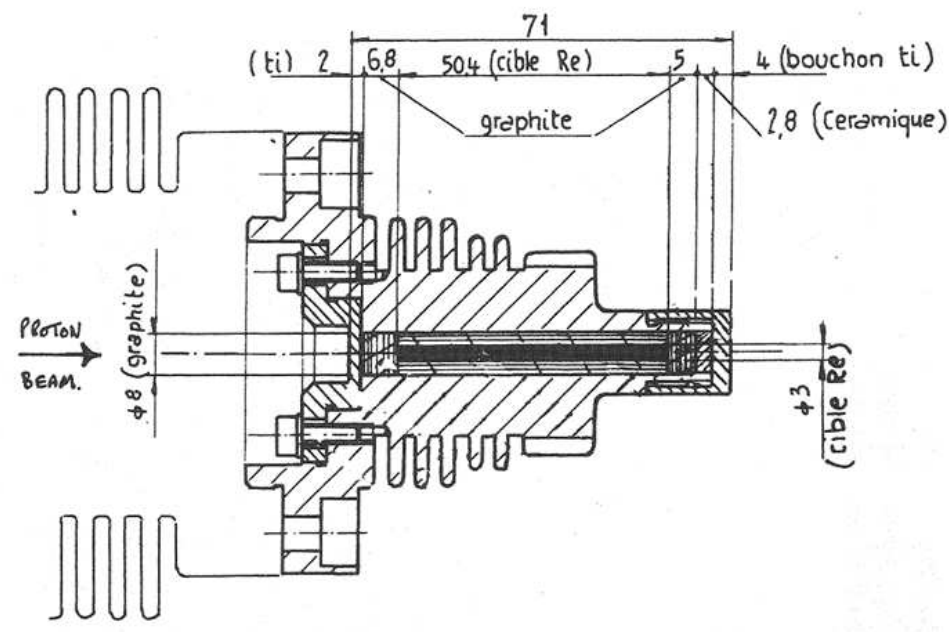

Figure 1.4: Schematics of one of the air cooled antiproton target used from the beginning of the 80's until 1987 [12].

graphite cylinder and encapsulated in a stainless steel envelope. Figure 1.5 shows the schematics including both target body and the lithium lens.

A thorough research concerning the possibility of using current pulsed targets was also done during these years. It was well known that passing a longitudinal current through the target at the time of the primary pulse impact could focus the produced negative-charged particles (including antiprotons) towards the downstream end of the target. This effect increased considerably the amount of antiprotons collected by the focusing device downstream [23, 24]. Furthermore, this procedure allowed the use of longer targets with lower Z, decreasing the high energy density deposition from the beam. Several tests were done by applying high current pulses (in the range 50 to $170 \mathrm{kA}$ ) throughout the target. Improvements of more than $50 \%$ in the yield with respect to previous designs were observed. However, failure of most of the targets was occurring in a time scale of hours or a few days (the antiproton production was dropping and the target had to be exchanged) probably due to target overheating and thermal shock. More detailed information about pulsed targets can be found in refs. [23, 24, 25, 26, 27]. The tests of pulsed targets were carried on until the late 80 's, but these practical difficulties lead to finally use passive targets, which shown lower antiproton production yield but were proven to be significantly more robust.

In July 1985 there was an incident while testing a current pulsed rhenium target using the design shown in figure 1.5. This design required the target to be close to the entrance of the lithium lens, restricting the space available for cooling the ex- 


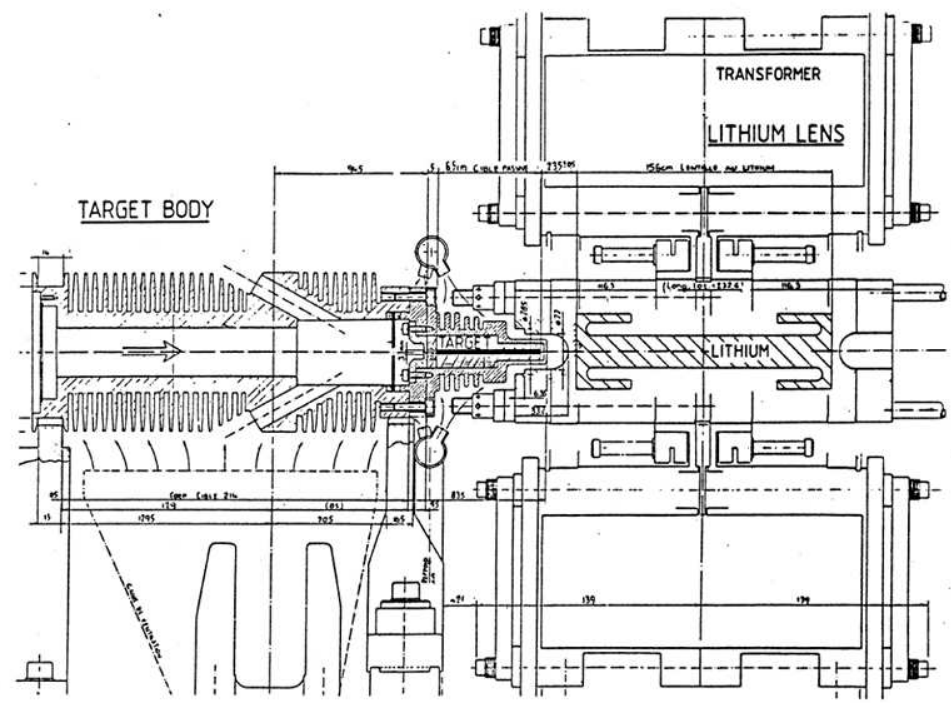

Figure 1.5: Schematics of the air cooled antiproton target inserted in the lithium lens, downstream focusing device used to collect the produced antiprotons until the 90s. This focusing device was substituted later by the magnetic horn shown in figure $1.8[24,28]$.

ternal envelope as can be seen in figure 1.4. Failure of the stainless steel enevelope at the downstream window occurred due to target overheating and fatigue damage. The failure of the envelope led to the release of rhenium spallation products and the spread of radioactive material. After this incident, titanium alloy grade 5 (Ti-6Al-4V) was used for the external envelope. Besides, new guidelines were established for futures designs, including the use of a closed-loop cooling system and a local secondary containment [29].

\subsubsection{Current Target Design}

In 1987 a new target design for initial operation of ACOL following the new suggested guidelines was proposed [27]. This design consisted of a water cooled target and it is the one that has been in operation to nowadays [23, 30]. Figure 1.6 shows a schematic of the full target assembly. As in previous designs, the target core is embedded in a graphite cylinder, which is sealed within a double-walled, water-cooled, titanium alloy envelope. The closed-loop water cooling system provides a significantly more robust design, reducing the risk of envelope failure due to overheating. Furthermore, titanium alloy has been proven to have a very good performance against thermal fatigue as well as to withstand the impact of high intensity proton beams [10]. 


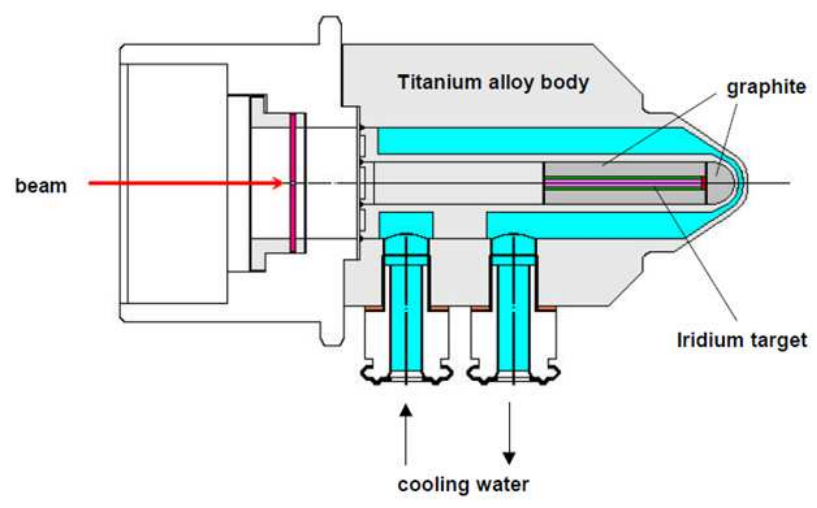

Figure 1.6: Schematics of the water cooled antiproton design used from 1987 until present days.

Figure 1.7 shows the complete target body and Li lens assembly used in 1987 [23, 31]. As in the previous air-cooled design (figure 1.5), the downstream head of the target was inserted in the Li lens. It is important to mention that the Li lenses were substituted by a magnetic horn during the 90's [28]. The later focusing device is more robust and easier to exchange, although there is a $20-40 \%$ decrease in antiproton collection yield compared to Li lens [11]. Figure 1.8 shows the watercooled target body and magnetic horn assembly, which design is the one that is still currently used. Figure 1.9 shows a picture of the current status of the antiproton target and horn in the AD-Target area.

Up to 11 target designs based on the water-cooled concept shown in figure 1.6 were proposed during 1987 and 1989 [32]. These different designs had slight differences in the external body geometry as well as different core target materials. Again copper and iridium were proposed. The final choice was a passive target of $3 \mathrm{~mm}$ in diameter by $55 \mathrm{~mm}$ in length made of iridium. This design has been kept since the early 90 's and is currently in operation. A picture of the exploded view of the target assembly is shown in figure 1.10.

The iridium rod is divided into six parts, five of them $10 \mathrm{~mm}$ long and one $5 \mathrm{~mm}$ in length. The total length of the rods is optimized according to the proton inelastic scattering length $\lambda_{p}$ and antiproton reabsorption length $\lambda_{\bar{p}}$. For the considered energies and material densities $\lambda_{p} \sim \lambda_{\bar{p}} \sim 50 \mathrm{~mm}[11]$.

In some of the designs, iridium rods of $2 \mathrm{~mm}$ in diameter are cladded in copper or nickel to an external diameter of $4 \mathrm{~mm}$ and then pressed into the graphite matrix in an effort to constraint the expected iridium fragments and limit the decrease of the iridium density as a consequence of the stress-wave damage [11]. In addition, the iridium pieces are embedded in the graphite together with a $1 \mathrm{~mm}$ of a $\mathrm{Al}_{2} \mathrm{O}_{3}$ end 


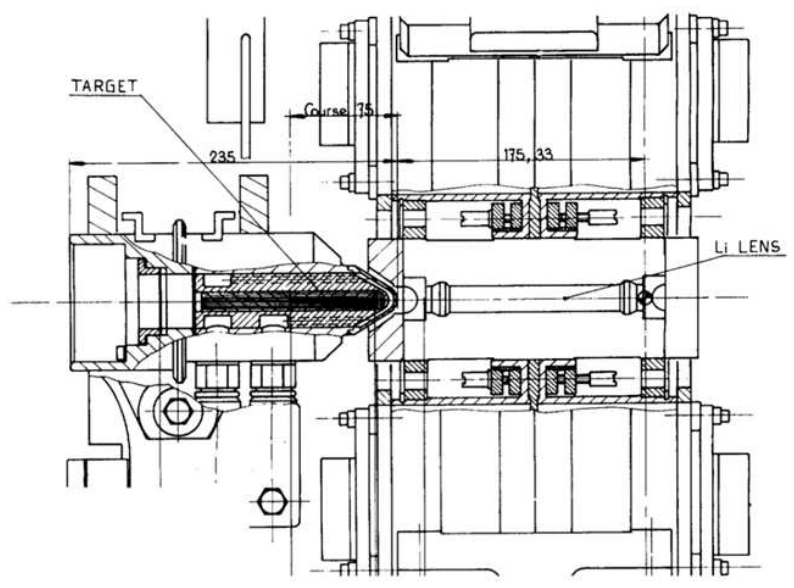

Figure 1.7: Schematics of the water-cooled antiproton target inserted in the lithium lens.

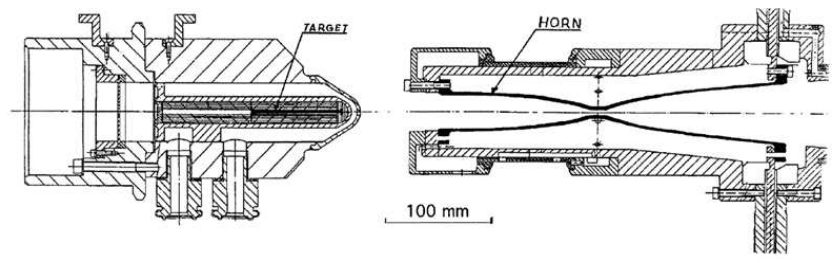

Figure 1.8: Schematics of the target/magnetic horn assembly, used for the antiproton production and focusing from the early 90 's until present.

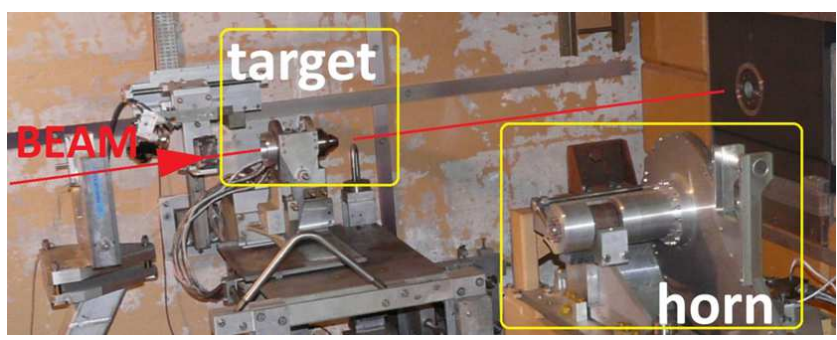

Figure 1.9: Picture showing the current AD-Target and magnetic horn. The proton beam comes from the left of the image and collides with the target. It must be mentioned that in the picture the horn is transversely displaced compared to the operation position, in which the horn and target are in line with the proton beam, as shown in Figure 1.8 [32]. 


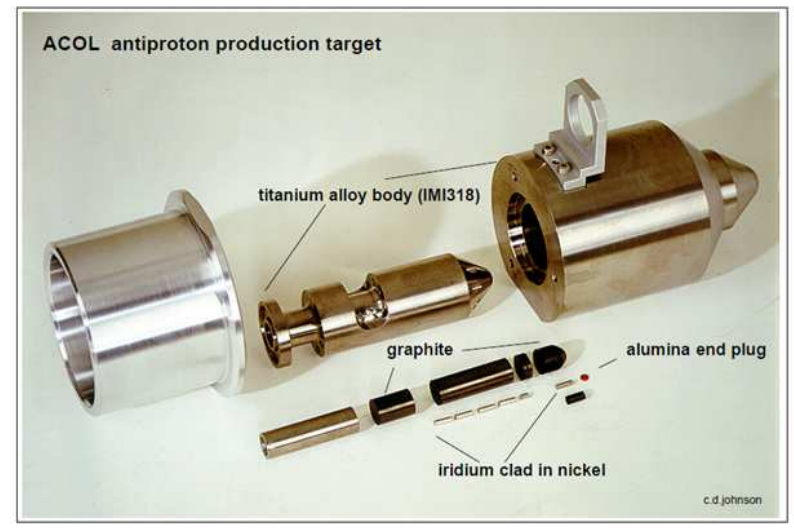

Figure 1.10: Picture of the exploded view of the current water-cooled antiproton target [30].

plug. This plug was in fact added into the former design right after the rhenium target incident of 1985 [19]. The cooling water is circulating through 8 radial holes gun-drilled in the internal titanium housing. A nominal water flow of $0.6 \mathrm{l} / \mathrm{s}$ at 10 bars is used to evacuate the energy deposited in each pulse. 


\subsection{Main Inherent Concerns and Operational Experience of the CERN's Antiproton Target}

From the technical notes and literature review carried out, it can be concluded that two main phenomena were identified as the major damaging issues during target operation; (i) stress waves as a consequence of the sudden increase of temperature in the target material during each pulse impact and (ii) radiation damage. These phenomena led to target failure due to a loss of effective density in the central part of the target rod, which reduced p-target interaction and antiproton production to the point that the target had to be replaced periodically to maintain the desired performance [23]. In addition to this, stresses and fatigue damages in the target containment and windows were reported, in particular with the second air-cooled design and its envelope made of stainless steel (figure 1.4) which, as described already, failed during some tests on pulsed targets in 1985.

\subsubsection{Sudden Rise of Temperature and Stress Waves in the Target Core}

The adiabatic increase of temperature due to beam impact is a function of the energy deposited in the target core, and is mainly influenced by the density and specific heat of the material. Lower density targets, as copper, showed an estimated increase of temperature per pulse in the order of $800{ }^{\circ} \mathrm{C}$ [19], while with higher density targets, such as tungsten or iridium, it was estimated up to $2000{ }^{\circ} \mathrm{C}$. These temperatures, even if they are considerably high, are lower than the melting temperatures of tungsten or iridium. However, temporary melting of the central part of the copper target was a reported possibility in the air-cooled designs as they worked at higher steady state temperature [33]. Nevertheless, the small dimensions of the target and the external cooling lead to an effective heat diffusion that makes the temperature to drop very fast after each pulse. For a pulse repetition rate of $2.4 \mathrm{~s}$ in the air-cooled design, $300{ }^{\circ} \mathrm{C}$ steady state temperature was estimated for copper, while $600{ }^{\circ} \mathrm{C}$ for iridium [19].

It was already reported, even from early operation in the late 70's, that the sudden increase of temperature in the target material during the beam impact would produce a rapid and non-uniform thermal expansion leading to a sudden increase in pressure and dynamic stresses. This effect was commonly identified as "shock waves" in old publications and AD-Target related technical reports [17, 34, 35]. However, in formal terms, they are not shock waves, as it is well established in the literature that a shock wave is characterized by a sharp discontinuity in pressure, density and temperature across its front [36]. As described in the present thesis, these conditions are not reached in the AD-Target and they are not expected as long as melting or vaporization of the target material does not take place. Nevertheless, the order of magnitude of the dynamic stresses and pressure reached 


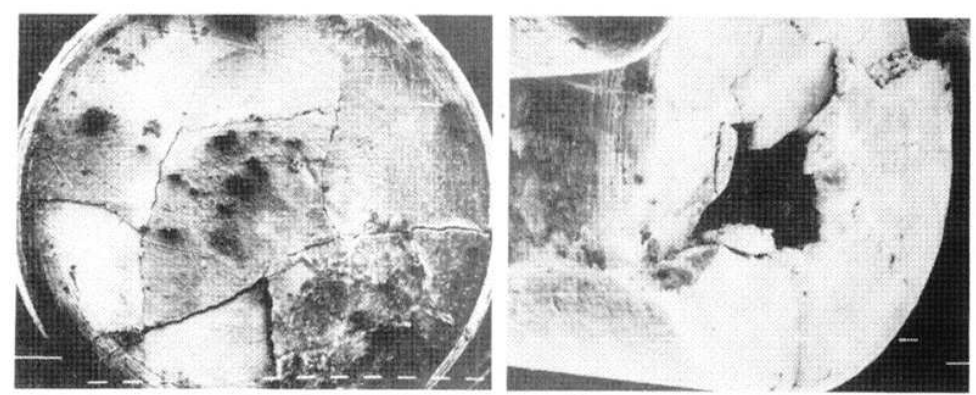

Figure 1.11: Pictures of the state of a tungsten rod after an irradiation test in 1979 [12].

inside the target material is largely above the material's yield and tensile strength, leading to plasticity and eventually cracks propagation and fracture. First target tests carried out during the late 70's already reported damage induced by beam impact [37]. Figure 1.11 shows the fracture along the cylinder axis of an irradiated tungsten target in 1979. The fracture of the material and permanent change of its density was reported as significantly reducing the antiproton production.

A substantial effort was made during 1978-1988 in order to identify the relevant parameters and select the most suitable materials and designs to withstand this effect. As a first approximation, it was considered that the resistance of the material to thermal stresses could be assessed by the magnitude of the factor:

$$
f=\sigma_{t} / \alpha E
$$

Which minimization is sought, i.e. it is desirable a material with high tensile strength $\sigma_{\mathrm{t}}$, low thermal expansion coefficient $\alpha$, and low Young's modulus E [37]. The density of the material also plays an important role, since the higher the material's density, the higher the beam energy deposited in the target and therefore the increase of temperature for the same primary proton beam.

In addition, toughness was considered to be an important property from the point of view of crack growth by the induced stresses. For this reason, special attention was paid to the brittleness of the material and to the brittle-ductile transition. In this context, metals with face-centred cubic (fcc) crystal structures, such as copper, which are ductile at low temperatures, were considered to have a better response than body-centred (bcc) metals. This was one of the main drawbacks of tungsten, which presents bcc structure and is prone to brittle fracture at temperatures below $300{ }^{\circ} \mathrm{C}$. Tungsten-rhenium alloys were proposed as the brittle-ductile transition temperature in these alloys decreases below room temperature [38]. Despite having presenting a fcc structure, Iridium shows an exceptionally brittle response [39]. However, it seems it had a better stability than tungsten under thermal waves 
since it was the material finally selected [11]. A detailed discussion of properties of candidate materials for AD-Target can be found in section 1.5 of this Chapter.

Finally, special attention was focused on the dynamic stresses induced by wave propagation from the the target core to the containing matrix, which in some designs was made of ceramics $\left(\mathrm{Al}_{2} \mathrm{O}_{3}\right)$ instead of graphite. Calculations of wave propagation using REXCO code [40] were carried out in 1985 for different target core/containing matrix material combinations [41]. These calculations shown the importance of the specific acoustic impedance of target material/matrix interfaces. The study was made under the consideration that any significant reflection of the wave energy into the target material at the insulator interface would lead to high radial stresses in the target. For this reason, the acoustic impedance mismatch between the core target material and its containing matrix should be as small as possible. This phenomenon is studied in detail in Chapters 2 and 3 (2.8.3 and 3.5) of the present thesis.

\subsubsection{Radiation Damage}

The second main concern regarding the antiproton production yield of the ADTarget reported in the literature is radiation damage induced during operation. Some reports were found in the literature assessing this issue and identifying it as the main cause for an effective decrease of up to $20 \%$ in antiproton production observed after periods of weeks of machine operation in the 80's [20, 42, 19, 34]. The reason for this decrease was identified in the radiation induced void formation inside the target material and subsequent swelling and effective loss of density. Nonetheless, as it will be shown in this thesis, recent numerical and experimental results presented in Chapter 3 and 4 suggest that this decrease of yield was caused by stress wave induced damage rather than by radiation damage itself, which would be more relevant in longer term operation.

Nevertheless, it is well known from nuclear reactor technology that energetic neutron collisions with metal atoms create dislocations in the crystal structures known as "Frenkel pair", in which the hit atom leaves its place in the lattice, creating a vacancy, and lodges nearby in the crystal, becoming an interstitial. The vacancies tend to diffuse through the material to form voids or empty spaces. In addition, formation of gases such as $\mathrm{Ar}, \mathrm{Ne}, \mathrm{Kr}, \mathrm{Xe}$ as consequence of spallation reactions or He by $\alpha$ - reactions contributes significantly to void growth as they diffuse easily inside them, forming bubbles. Macroscopically, the net effect is that the material swells. Formation of such gases also turns out to be relevant for the detriment of mechanical properties of the material, especially when the gases migrate towards the grain boundaries, making the material brittle.

In the AD-Target, in the same way as in nuclear reactors, the core material is subjected to collisions of energetic particles. The effective fluence experienced by 
the target, defined as the number of particles passing per unit area, will be the sum of direct primary protons fluence as well as significantly large number of secondary energetic particles, mainly pions, kaons, neutrons and protons, produced in the intranuclear cascade when the primary protons interact with the target core.

The relation between fluence and effective swelling and embrittlement in a material are not trivial, even less in the case of high energy proton irradiation, for which substantially less experience as with neutron exists. Swelling is a function of the microscopic void formation and growth processes which are strongly influenced by the target metallic crystal structure, operation temperature, stresses on the material and compressibility of the containing matrix. One of the most influencing parameter is the irradiation temperature. In that sense, it is considered that swelling is enhanced at intermediate operational temperatures, normally $0.3 \mathrm{~T}_{\mathrm{m}}<\mathrm{T}<0.5 \mathrm{~T}_{\mathrm{m}}{ }^{\circ} \mathrm{C}$, where $\mathrm{T}_{\mathrm{m}}$ is the melting temperature of the material. This behavior is common for almost all metals and it is due to the intrinsic nature of void formation as combination of void density (void nucleation) and their size distribution (void growth). At low temperatures, low defect mobility limits the displacement of vacancies to form voids, while at high temperatures voids are not able to grow sufficiently due to the re-emission of vacancies [43]. This dependency is especially relevant for the target design as it would be desirable that the target material works outside of this dangerous range of intermediate temperatures. However, in practice, this is difficult to achieve, since first of all the adiabatic increase of temperature in the target cannot be controlled (and as it will be seen, is in the order of $2000{ }^{\circ} \mathrm{C}$ ), and in addition, it is hard to make an accurate prediction of the target operating temperatures due to uncertainties in the state of target core and matrix material, which would strongly influence the thermal contact conductance between elements.

\subsubsection{Concerns on the Target Envelope}

In the previous section, some of the concerns of the target material which directly reduce the antiproton yield during operation have been exposed. In addition to these concerns, special attention has to be paid to the design and material choice of the external containment, in particular to the downstream window which is subject to high temperature fluctuations as well as radiation damage from the non-scattered primary proton beam. These concerns were accentuated by the use of high density pulsed targets as iridium, tungsten or rhenium due to their higher working temperatures. In the first target designs, aluminium alloy 6061 as well as stainless steel were used. However, the latter material was not suitable for working with high-density targets due to its poor fatigue characteristics at high temperatures, which led to envelope rupture at the downstream windows when using rhenium targets in the design shown in figure 1.4 [29]. 
A discussion of performances of different candidate materials for the target envelope, such as stainless steel, aluminium alloy 6061, titanium alloy Ti-6Al-4V and Ni-Co-Cr alloy (Nimonic 105) was done after the rhenium target incident [44]. The performance of these materials depend on the working temperature and therefore on the efficiency of the target cooling. For these calculations, steady state temperature of the downstream window of $550{ }^{\circ} \mathrm{C}$ was assumed, considerably high due to the inefficient air cooling of the design at that time. Calculations showed that, as expected, stainless steel would fail relatively quick, while titanium alloy would be acceptable up to $400{ }^{\circ} \mathrm{C}$ but it is under its limit conditions at $550{ }^{\circ} \mathrm{C}$. Nimonic 105 showed the best characteristics up to $800{ }^{\circ} \mathrm{C}$. The final choice for the subsequent designs was titanium alloy Ti-6Al-4V as discussed above. Explanations of why the final choice was Ti-6Al-4V instead of Nimonic (which, according to this report [44] behaves better) have not be found. It must be said in any case that, due to the shift to water cooling in the later designs, the temperatures reached at the downstream windows were considerably lower. Moreover, the shape of the external envelope of the water cooled version was properly designed in order to avoid stress concentrators and to reduce fatigue effects. In this context, spherical external surfaces in the end windows are more efficient to withstand the applied thermal stresses [44]. This improvement can be observed in comparison to the previous air-cooled designs (figure 1.4), where square shapes were used. The second reason for the use of spherical shape in the downstream external surface was to facilitate the target fitting into the lithium lens as shown in figure 1.7.

\subsection{Comparison with other High Power Target Facilities}

As already mentioned in section 1.2.1, a focused and intense primary proton beam is required in addition to the selection of a high density material to increase the antiproton production in the target. The primary beam in recent operational years consists of $1.5 \cdot 10^{13} \mathrm{ppp}$ (protons per pulse) with a traversal Gaussian size at $1 \sigma$ of $1 \mathrm{~mm}$ in the horizontal and $0.5 \mathrm{~mm}$ in the vertical plane. As explained in detail in section 2.3, the energy deposition has been recently calculated using FLUKA [45] Monte Carlo simulations for the iridium target in operation, showing that $1.34 \mathrm{~kJ}$ is deposited only in the iridium rod in each pulse, leading to a volume averaged energy deposition of $3400 \mathrm{~J} / \mathrm{cm}^{3}$ per pulse and a maximum deposited energy density of $7500 \mathrm{~J} / \mathrm{cm}^{3}[10]$.

Very high energy deposition density differentiates the antiproton target from other targets or spallation sources, which may have higher total power, but distributed along much larger volumes and deposited in longer beam bursts (affecting significantly the dynamic response as shown in further chapters). Table 1.1 shows, for instance, other existing high power targets operating around the world. The table summarizes the peak of energy density deposited in them. As can be seen in the 
Table 1.1: Summary of existing target facilities working with high energy density deposition.

\begin{tabular}{|c|c|c|c|c|c|}
\hline Facility & $\begin{array}{l}\text { Place (In } \\
\text { operation } \\
\text { from) }\end{array}$ & $\begin{array}{c}\text { Solid target } \\
\text { material }\end{array}$ & $\begin{array}{l}\text { Designed } \\
\quad \text { for } \\
\text { producing }\end{array}$ & $\begin{array}{c}\text { Peak } \\
\text { deposited } \\
\text { energy } \\
\mathrm{J} / \mathrm{cm}^{3} \text { pulse }\end{array}$ & $\begin{array}{l}\text { Pulse } \\
\text { length } \\
\mu \mathrm{s}\end{array}$ \\
\hline $\mathrm{AD}$ & $\begin{array}{c}\text { CERN } \\
(1987)\end{array}$ & Iridium & Antiprotons & 7500 & 0.4 \\
\hline CNGS & $\begin{array}{l}\text { CERN } \\
(2007)\end{array}$ & Graphite & Neutrinos & 1500 & $10[46]$ \\
\hline $\mathrm{n} \_\mathrm{TOF}$ & $\begin{array}{l}\text { CERN } \\
(2001)\end{array}$ & Lead & Neutrons & 20 & 0.005 \\
\hline $\begin{array}{l}\text { Pbar } \\
\text { Source }\end{array}$ & $\begin{array}{c}\text { Fermilab } \\
(1985)\end{array}$ & Inconel & Antiprotons & $13350[47]$ & 1.6 \\
\hline SNS & $\begin{array}{c}\text { ORNL-US } \\
(2006)\end{array}$ & Mercury & Neutrons & 13 & $0.7[48]$ \\
\hline ISI & $\begin{array}{l}\text { RAL-UK, } \\
\text { (1985) }\end{array}$ & Tungsten & Neutrons & 100 & $0.8[49]$ \\
\hline $\mathrm{BDF}$ & $\begin{array}{l}\text { CERN } \\
(2025)\end{array}$ & $\begin{array}{c}\text { Tungsten- } \\
\text { TZM }\end{array}$ & $\begin{array}{l}\text { Hidden } \\
\text { Particles }\end{array}$ & 840 & $10^{6}[50]$ \\
\hline ESS & $\begin{array}{l}\text { Lund, } \\
\text { Sweden } \\
(2020)\end{array}$ & Tungsten & Neutrons & 370 & $2860[49]$ \\
\hline
\end{tabular}

table, only the Pbar Source operated at Fermilab (US) for the Tevatron machine reached energy depositions comparable to the AD-Target. Nevertheless, even in this case the energy was deposited in a longer time than in the AD-Target $(1.6 \mu \mathrm{s}$ vs $0.43 \mu \mathrm{s})$. Some details of the Pbar Source are included in the next section.

\subsubsection{The Pbar Source at Fermilab}

The only other antiproton production target existing in the world was the Pbar Source at Fermilab (US), which was in operation from 1985 to 2011, providing antiprotons for $p-\bar{p}$ collisions for the Tevatron collider [51, 52]. This target was receiving a beam of $126 \mathrm{GeV}$ from the Main Injector. The produced antiprotons were collected and focused using Li lens at an optimum $\bar{p}$ momentum of $8.9 \mathrm{GeV} / \mathrm{c}$ to subsequently inject them into the Debuncher and Accumulator Rings. After accumulating a sufficient number of antiprotons, they were sent to the Recycler for additional cooling and accumulation before injecting into the Tevatron, where 


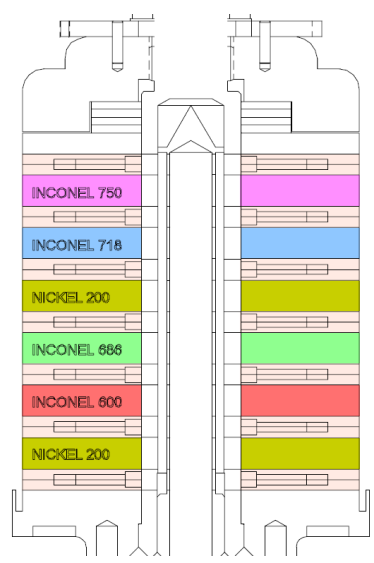

(a)

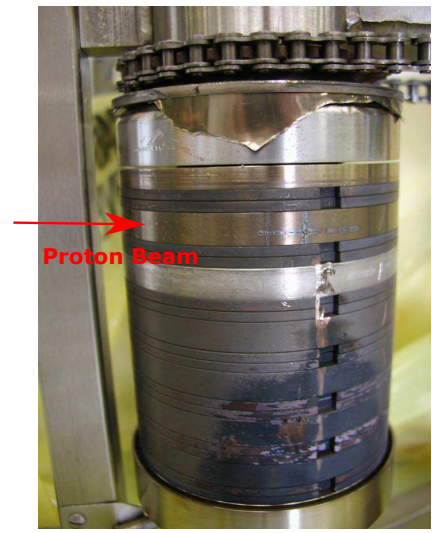

(b)

Figure 1.12: (a) Schematics of the Fermilab Pbar Source Target consisting in a stack of disks. (b) Picture of an irradiated target showing material depletion by melting [53].

they were accelerated to $900 \mathrm{GeV}$ and collided with protons achieving collision energies of $1.8 \mathrm{TeV}$.

The conceptual design of the Pbar Source Target was significantly different from the CERN one, consisting of a stack of disks of different materials with the vertical axis perpendicular to the primary proton beam, as shown in figure 1.12-(a). By raising and lowering the target disks, it was possible to change the target material and by moving the target horizontally it was possible to change the material thickness exposed to the beam. In addition, the target was rotated between beam pulses to spread the material depletion and damage uniformly around the circumference. This design was very convenient since the high primary beam focusing was frequently producing permanent damage and even melting the target. This concept, however, is not compatible with the configuration of the CERN AD-Target area since it requires to have the targets inside a vault in order to avoid the release of radioactive material from the potentially damaged disks.

In the same way as in the AD-Target, different materials were tested during the 26 years of operation of the Pbar source. Initially, tungsten or tungsten alloys such as tungsten-rhenium. Later on, experiences with severe damage to the tungsten targets prompted to a shift to copper, nickel (Nickel $200^{\circledR}$ ) or different grades of INCONEL $^{\circledR}(600,625,686, \mathrm{X}-750)$. Two main problems were reported as the cause of target failure during operation. First, the rapid heating and expansion of the target material and successive waves causing mechanical damage to the target. Second, a molten channel formed in the target, in particular when using copper, nickel or INCONEL ${ }^{\circledR}$ targets [47], which have significantly lower melting temperatures. It is important to note the different primary beam characteristics of this 
target, much more focused $\left(0.2 \mathrm{~mm}\right.$ radius at $1 \sigma$ for an intensity of $1.8 \cdot 10^{12} \mathrm{ppp}$ [51]) in comparison with the CERN's AD-Target. In addition, the significantly different target geometry makes difficult the extrapolation between the dynamic conditions reached in this target and the AD-Target, since, as explained in Chapter 3 , geometry greatly influences the wave propagation and magnitude of the reached dynamic stresses.

\subsection{Candidate Core Materials for the Future AD-Target Design}

In this section, a description of materials which are considered as candidate for the future AD-Target core is presented, including a literature review regarding its properties of interest. These materials are tested under proton beam impacts in the HiRadMat experiment described in Chapter 4, in which are they exposed to equivalent conditions as the ones present in the AD-Target.

As mentioned in this introduction, there are some particular properties required by the antiproton production and target operation. The first of these properties is the highest available density, in order to reduce the target length and to be as close a possible to a punctual source. Second property is a very high melting point (above $2000{ }^{\circ} \mathrm{C}$ ). Target melting during operation could be catastrophic since a change of phase and fast re-solidification could lead to the appearance of hollows in the core, which would bring multiple uncertainties. These two requirements narrow the selection to refractory metals, which are the only materials that present these properties.

In addition, other mechanical properties such as high strength and stability at high temperature are sought. Available information of the materials is also considered as an important variable, in particular regarding the knowledge of their behavior at high strain rate and temperature, as well as literature regarding their response to radiation damage. Finally, applicability of the candidate materials to other target facilities in the word is also valued, so the findings of this thesis could be extended to them. Based on these specifications, the following materials are studied:

\subsubsection{Iridium}

The inclusion of this material in the studies of this thesis is more than well justified by the fact that this is the current target core material, selected by its high density in the last AD-Target design at the late 80 's. Iridium is a very rare metal belonging to the platinum group, which possess a unique combination of properties such as very high density (it is the second densest element after osmium with a density of $\left.22.46 \mathrm{~g} / \mathrm{cm}^{3}\right)$, high melting point $\left(2450{ }^{\circ} \mathrm{C}\right)$, excellent oxidation and corrosion resistance and high strength even at high temperature $[54,55,56]$. It is also the 
second hardest metal with a young modulus of $530 \mathrm{GPa}$, only exceeded by osmium. Despite its particular and interesting properties, its elevated price makes that it only exists in a few applications as pure structural material, always in the high technology field, such as material for nozzles of rockets, capsules for radioisotope heat sources in spacecrafts, or the target core material for antiproton production as seen in this thesis $[57,58]$.

Due to its rareness, elevated price and restricted applications, only few studies of mechanical stress-strain properties of pure iridium have been found in the literature. The tensile properties in static conditions and up to $2000{ }^{\circ} \mathrm{C}$ were studied in ref. [59]. This study shows the high yield strength of iridium and how it is strongly temperature dependent, estimating a yield strength of $990 \mathrm{MPa}$ (at $0.1 \%$ strain) at room temperature and its drop down to $40 \mathrm{MPa}$ at $1600{ }^{\circ} \mathrm{C}$. In the same way, the observed elongation to break varied from $1.1 \%$ at room temperature up to $10.2 \%$ at $1500{ }^{\circ} \mathrm{C}$. Iridium, unlike most of others fcc metals, exhibits a brittle-ductile transition and only moderate ductility at high temperatures. The brittleness of iridium at low temperature (in comparison to the rest of fcc metals) has been studied in detail in ref. [39], suggesting that below $1000{ }^{\circ} \mathrm{C}$ iridium fails intrinsically by BIF (brittle intergranular fracture). However, this is a matter of discussion in the literature. Further studies have been performed on iridium fracture mode (intergranular and transgranular) and its relation to impurity content [60, 61], including tests on iridium single crystals. These studies claim that BTF (brittle transgranular fracture) is the inherent fracture mode of iridium, while BIF may be considered as an impurity-induced fracture mode.

Concerning the dynamic behavior of pure iridium, not a single study has been found in the literature apart from a few publications on stress-strain properties under impact loading of DOP-26 (an iridium alloy containing $0.3 \%$ of $\mathrm{W}$ and approximately $40 \mathrm{ppm}$ of Th to improve grain boundary cohesion at high temperatures), mainly motivated by the use of this material as cladding for radioisotope thermo-electric generator $[62,63,64,65,66]$. It is for this lack of material properties that a dynamic characterization campaign of pure iridium was launched in the context of this thesis (described in section 2.7.3 of Chapter 2). This study, published in ref. [67], included analysis of different modes of fracture of Ir, its dynamic behavior at high temperature and strain rates, and strength model extraction.

\subsubsection{Tungsten}

Tungsten is a very well known refractory bcc metal with numerous applications as structural material in industry due to its high density $\left(19.3 \mathrm{~g} / \mathrm{cm}^{3}\right)$, high melting point $\left(3420^{\circ} \mathrm{C}\right)$, and particular mechanical properties such as hardness and high tensile strength. Polycrystalline tungsten has a young modulus of $400 \mathrm{GPa}$ and tensile strength above $1500 \mathrm{MPa}$ (at room temperature) [68]. Its high melting point and density makes it common in numerous applications, including incandescent 
light bulbs filaments, X-rays tubes, electrode in TIG welding, as well as radiation shielding and medical applications [69]. In addition, and despite its brittleness at low temperature, its combination of high strength and high density makes the material very attractive for certain specialized military applications such as kinetic penetrators employed to defeat armor, making it widely studied under dynamic laoding [70, 71]. In this context, tungsten is present in many applications in form of the so called tungsten-heavy-alloys (WHAs), which are sintered materials based on pure tungsten $(>90 \%)$ with a $\mathrm{NiFe}$ or $\mathrm{NiCu}$ binder phase. WHAs provide a very good compromise between high density and mechanical properties, being less brittle than pure tungsten and with a much better workability. Unfortunately, these alloys are not applicable to the AD-Target since they start to form liquid phases below $1500{ }^{\circ} \mathrm{C}$ due to the addition of $\mathrm{Ni}, \mathrm{Fe}$ or $\mathrm{Cu}$ [72]. Nevertheless, it has been shown that the behavior of the pure tungsten dominates the dynamic response of these alloys [73], substantially motivating the study of its dynamic behavior in pure state $[70,71,74]$, in addition of course to the very widely dynamically studied WHAs $[75,76,77]$. Up to three different studies including strength model characterizations of pure tungsten at different range of strain rates and temperatures have been found in the literature [78, 70, 79].

The properties of polycrystalline tungsten are greatly influenced by its microstructure (manufacturing process, grain size and shape, cold work, annealing...) and presence and concentration of impurity elements, its solubility and segregation to grain boundaries [69]. This was already observed in ref. [68], which provided a very wide database of mechanical properties of tungsten such as yield strength and tensile strength at different temperatures for 32 configurations, including different impurity contents, manufacturing method (sintered/arc melted), geometries (rod, sheets, wire) and material conditions (cold worked, stress relieved, recrystallized).

It is important to mention that polycrystalline tungsten is intrinsically brittle at room temperature, especially under tension, due to its high ductile-to-brittle transition temperature (DBTT). Below this transition temperature, tungsten fractures with very little macroscopic deformation. Moreover, a very spread range of temperatures over which the ductile-brittle transition occurs has been reported in the literature since it is highly dependent of the nature of the material, such as grain size, impurity concentration and thermo-mechanical processing [80]. In any case, very low ductility in tension has been observed below $150{ }^{\circ} \mathrm{C}$. The DBTT is as well very dependent on the loading conditions such as strain rate as shown in ref. [81], in which hot forged tungsten was tested under shock loading at $22{ }^{\circ} \mathrm{C}$ and $400{ }^{\circ} \mathrm{C}$. Radiation damage also plays a very important role on the shift of the DBTT as demonstrated in refs. [82, 83], which showed important tungsten embrittlement after $800 \mathrm{MeV}$ protons irradiation.

Research on tungsten and tungsten alloys (excluding WHAs which are limited in some cases by its considerable lower melting point) in the nuclear field has been greatly enhanced in the last decades as it is the main candidate material for the 
divertor and plasma-facing-armor of the Tokamak fusion reactor of ITER (International Thermonuclear Experimental Reactor) or DEMO (DEMOnstration Power Station) [84]. These activities push the search for new tungsten alloys as well as manufacturing methods that meet the properties necessary for such application, like high thermal conductivity, high temperature strength and stability, and high recrystallization temperature. In this context, research on radiation induced damage in tungsten and its alloys is also taking place due the enormous neutron load under which this component would work [85], since radiation can produce a shift of DBTT in the range of several hundreds of degrees.

From the point of view of improving the brittleness of tungsten by allowing it without compromising its high melting point, so far only rhenium is known to improve its ductility by its solid solution [86, 85]. For this reason, tungstenrhenium could have been a good candidate for core material of the AD-Target and it was even proposed in some CERN's internal notes during the 80's [38]. However, several recent studies had shown that the initial more ductile behavior of tungsten-rhenium in comparison to pure tungsten rapidly vanishes when exposed to radiation $[87,88,89]$. This fact discouraged the inclusion of $\mathrm{W}$-Re in benefit to other tungsten based material such as tungsten dopped with lanthanum, as described in the next section.

In any case, the inclusion of pure tungsten as a candidate material for the ADTarget was due to, in addition to its very suitable density, the experience and interest on this material that, as just described, already exists in the scientific community. Together with the good availability of strength models of this material and the fact that most of the hydrocode calculations performed in Chapter 4 consider it as core material. In addition, tungsten's combination of high density and strength at high operating temperatures makes it very suitable for neutron spallation targets. For example, pure tungsten has been used as core material for the ISIS spallation source at RAL (Rutherford Appleton Laboratory) [90] and it is a possible candidate for the future design of CERN's n_TOF spallation target [91] and the target of the Neutrino Factory [92]. Furthermore, pure tungsten is the baseline candidate material for other future high powered targets such as the one of the European Spallation Source (ESS) in Lund [93] and the potential BDF (Beam Dump Facility) at CERN [94]. These applications also support the inclusion of pure tungsten in the numerical and experimental studies of this thesis, since the information of its behavior under proton beam impacts could be transversely applied to these other projects. 


\subsubsection{Tungsten doped with Lanthanum Oxide}

As already introduced in the previous subsection, lot of research on tungsten based materials is currently taking place due to its multiple application and, in particular, to fusion reactors. The objective is to find an improved tungsten material which presents high crack resistance under the extreme thermal conditions present in such reactor. In this context, it was discovered that pure tungsten doped with $1 \%$ of $\mathrm{La}_{2} \mathrm{O}_{3}$ improves significantly its mechanical properties at high temperature such as tensile and creep strength due to an increase of its recrystallization temperature [95]. In addition, dispersion of a second phase such as oxide particles could be an effective method of improving the resistance against irradiation hardening due to introduction of defect sinks (e.g., grain boundaries and dislocations) which avoid accumulation of clusters of irradiation-induced defects [96]. Irradiation campaigns had shown that tungsten doped with $\mathrm{La}_{2} \mathrm{O}_{3}$ is expected to maintain favorable high-temperature strength up to 0.5 dpa [96]. Furthermore, experiments exposing $\mathrm{W}$ and $\mathrm{W}-\mathrm{La}_{2} \mathrm{O}_{3}$ to deuterium plasma shots showed that the latter presented a higher resistance to the development of micro-cracks (although observed macroscopic defects for $\mathrm{W}$ and $\mathrm{W}$-doped were almost similar [97]).

Nevertheless, it must be mentioned that the $\mathrm{W}-\mathrm{La}_{2} \mathrm{O}_{3}$ doped does not present any improvement of the DBTT with respect to pure tungsten or is even more unfavorable [98]. This fact limits the use of this material to only high temperature operations. In any case, it was considered interesting to add this material in the candidate list and to test it in the experiment shown in Chapter 4 since, in addition to the AD-Target, it can have similar applications as pure tungsten in spallation targets (previously described). Therefore, a comparison of the performance between pure and doped tungsten could be assessed.

\subsubsection{Tantalum}

Tantalum is a refractory bcc metal with density of $16.6 \mathrm{~g} / \mathrm{cm}^{3}$ and melting point of $3017^{\circ} \mathrm{C}$ [99]. It is well known for its corrosion resistant properties, in particular to acids. In addition, tantalum is very easy to work even at low temperature and despite its bcc crystal structure. Tantalum is relatively strong (young modulus at room temperature $186 \mathrm{GPa}$ ) but it presents at the same time a very ductile behavior when annealed, with a yield strength at room temperature around $170 \mathrm{MPa}$ and ultimate tensile strength of $280 \mathrm{MPa}$, showing elongations to break above $30 \%$ [100]. It has a recrystallization temperature in the range from $900{ }^{\circ} \mathrm{C}$ to $1200{ }^{\circ} \mathrm{C}$. Tantalum strength however can be significantly increased when cold worked, achieving yield strength up to $440 \mathrm{MPa}$ and ultimate tensile strength of $650 \mathrm{MPa}$ (at room temperature) [101].

Due to its high melting point and corrosion resistant properties is used for electrolytic capacitors, chemical process equipment such as heat exchangers, con- 
densers, furnaces as well as in specialized aerospace and military applications. In this context, dynamic properties of tantalum have been extensively studied since it is a common material in shaped charges liners in warheads and explosively forged projectiles [102]. First studies on temperature and strain rate dependence of the flow stress in tantalum can be found in ref. [103], which covered testing temperatures from $-196{ }^{\circ} \mathrm{C}$ to $530{ }^{\circ} \mathrm{C}$ and strain rates from $10^{-5} s^{-1}$ to $2 \cdot 10^{4} s^{-1}$. In ref. [104] tensile experiments were carried out on tantalum at room temperature in the strain rate range $10^{-4} s^{-1}$ to $3 \cdot 10^{3} s^{-1}$. Zerilli and Armstrong also showed interest on this material, using experimental data from refs. [103, 104] to obtain material parameters for their Zerilli-Armstrong (Z-A) strength model in ref. [105]. In addition, Chen and Gray [78, 106] also performed dynamic tests of pure tantalum in compression. In their study, quasi-static tests were performed at $-196{ }^{\circ} \mathrm{C}$ and at room temperature, and Hopkinson Pressure Bar tests from $1.5 \cdot 10^{3} s^{-1}$ to $5 \cdot 10^{3} s^{-1}$ and from $-196{ }^{\circ} \mathrm{C}$ to $1000{ }^{\circ} \mathrm{C}$. Parameters for the Mechanical-Threshold-Stress (MTS), Johnson-Cook (J-C) and Z-A models were extracted. All these strength models are described in detail in section 2.6.3 of Chapter 2 of the present thesis.

Other study on tantalum subjected to high shear strains at high strain rate, focused towards dynamic recrystallization processes, can be found in ref. [107], where tests were performed from $25{ }^{\circ} \mathrm{C}$ to $525^{\circ} \mathrm{C}$ and up to $5 \cdot 10^{3} \mathrm{~s}^{-1}$ by Hopkinson Pressure bar. A moderate strain-rate sensitivity while strong temperature sensitivity in the dynamic response was observed. Further studies on the microstructural processes behind the tantalum deformation behavior at high strain rate and temperatures can be found in refs. $[102,108,109,110]$. In addition, tantalum is recently studied in the context of multiscale modelling to predict its behavior at extremely high pressures, thanks to its widely available experimental data and its unique properties such as a bcc-structure stability at extremely high pressures. Some examples of multiscale modelling of tantalum can be found in refs. [111, 112, 113, 114].

Another property of tantalum that has been reported in the literature and which can be of great interest for the AD-Target core application is its high spall strength. In refs. $[115,116,117]$ different grades of tantalum specimens were subjected to spall gas-gun-driven experiments at room temperature, finding its spall strength around $5 \mathrm{GPa}$. These values were found as well in ref. [118] which compared two tantalum materials produced by different melting manufacturing processes, finding 4.7 and 5.4 GPa spall strength for the two tested materials. A more recent study can be found in ref. [119], in which the spall strength of tantalum as a function of strain rate and different grain size configurations (including single crystals) is shown. This work, besides being a great collection of data from previous spall experiments in tantalum, demonstrated the strain rate dependence of this property (the higher the strain rate, the higher the spall strength). Spall strength from 4 to $5 \mathrm{GPa}$ were found in the range of strain rates reached in the AD-Target core $\left(10^{4}-10^{5} s^{-1}\right)$. 
Several studies have been found in the literature addressing radiation damage in tantalum. First studies were performed during the 70's, motivated by the potential use of this material in fast breeder reactors or space reactors [120,121], while more recent studies are related to the the use of this material in spallation targets such as ISIS in RAL or ESS. Data of radiation damage induced by proton irradiation can be found in refs. [122, 123, 124, 125].

In ref. [120] pure tantalum was subjected to $2.5 \cdot 10^{22} \mathrm{n} / \mathrm{cm}^{2}$ and $4.4 \cdot 10^{22} \mathrm{n} / \mathrm{cm}^{2}$ in the range from 400 to $1000{ }^{\circ} \mathrm{C}$ in the Experimental Breeder Reactor II (EBR-II), reaching estimated damages in the order of 20-35 dpa. This study found a strong irradiation temperature dependence of swelling, reaching a maximum of $2.4 \%$ at $580{ }^{\circ} \mathrm{C}$. Negligible swelling was observed at temperatures below $400{ }^{\circ} \mathrm{C}$ and above $1000{ }^{\circ} \mathrm{C}$. In ref. [121] subsequent studies on neutron irradiated tantalum also in EBR-II are presented, including tensile tests and swelling measurements of samples irradiated up to $2.2 \mathrm{dpa}$ at temperatures from $380{ }^{\circ} \mathrm{C}$ to $680{ }^{\circ} \mathrm{C}$. Very low levels of swelling were observed, attributed to not reaching the incubation fluence for swelling onset. Regarding the tensile testing, specimens after irradiation showed an increase of yield strength from $400 \%$ to $600 \%$, associated with drop in the elongation to fracture from $40 \%$ (before irradiation) to $6 \%$ (when irradiated). Nevertheless, it was reported that, despite such limited uniform elongation, the fracture surface revealed a complete ductile rupture, nearly indistinguishable from the unirradiated specimen.

In later publications applied to neutron spallation targets tantalum was subjected to proton irradiation. In ref. [124] an extended summary of previous studies on radiation damage in tantalum and its alloys is presented, including a detailed analysis of competing radiation damage phenomena at different irradiation temperatures. In this context, it is claimed that when operating below $0.3 T_{m}$ (which is not the case of the AD-Target), hardening will be the dominating radiation damage effect, while void swelling should not be a problem with operating temperatures below $400{ }^{\circ} \mathrm{C}$ and above $1000{ }^{\circ} \mathrm{C}$. In ref. [125] mechanical tests on $800 \mathrm{MeV}$ proton irradiated tantalum that was part of the ISIS spallation target are presented. During operation, this material was exposed up to $11 \mathrm{dpa}$ at operating temperatures below $200{ }^{\circ} \mathrm{C}$. Mechanical tests and SEM (scanning electron microscope) investigations showed that Ta retained very high ductility after proton irradiation even at the highest doses, revealing typical ductile fracture surfaces when mechanically tested. Existing discrepancy between this large retained ductility in comparison with previous studies on proton irradiated tantalum [122, 123] was explained by the extremely low impurity content in the former. Radiation damage in tantalum is still under investigation by several works. In ref. [126] pure tantalum was recently irradiated by $200 \mathrm{MeV}$ protons in BLIP facility of Brookhaven National Laboratory up to $2 \mathrm{dpa}$ and at a irradiation temperature of $400{ }^{\circ} \mathrm{C}$. At the highest dose, yield strength of Ta increased from $160 \mathrm{MPa}$ (unirradiated) to $350 \mathrm{MPa}$, while a significant ductility was still retained [127]. 
Given the high density of tantalum, the extended research already existing in this material, and its multiple applications in target facilities, it was considered valuable to add this material in the $\mathrm{AD}$-Target candidate list, as well as in the experimental test presented in Chapter 4. In addition, it is important to note the much more ductile response (yield strength of $170 \mathrm{MPa}$ ) of Ta in comparison to the other candidate materials. Furthermore, tantalum, as already mentioned, presents very high resistance to spall. Given the fact that, due to the large level of stresses taking place in the AD-Target core, it is assumed that all of the candidate materials would experience plastic deformation in any case, it was considered interesting to introduce tantalum in the studies in order to compare possible failure mechanisms between materials with known different properties and ductility.

\subsubsection{Molybdenum and TZM}

Molybdenum is a bcc metal with a high melting point of $2620{ }^{\circ} \mathrm{C}$ and a density of $10.2 \mathrm{~g} / \mathrm{cm}^{3}$. This density is significantly lower than the one of the other considered materials and that would therefore affect the antiproton production if applied to the AD-Target as explained in section 1.2.1 of this chapter. This reduction would need to be compensated by an increase of the AD-Target core length, from the $55 \mathrm{~mm}$ currently used with iridium up to $100-120 \mathrm{~mm}$. This was the case of the copper targets used during the 80's. Nevertheless, and despite its reduced density which would lead to a less optimized collection of antiprotons by the downstream magnetic horn, it was considered interesting to add this material to the candidate list and experimental studies in order to assess the fact that a lower density material could have more chance to survive since the conditions on it would be less extreme. In addition, molybdenum and its alloys (such as TZM) are of interest in future $R \& D$ projects on targets at CERN, since it will be one of the target materials of the potential Beam Dump Facility [94].

Due to its high melting point and good thermal and mechanical properties, molybdenum has multiple of applications such as lighting industry, glass melting electrodes and high-temperature furnaces [128]. As most of the other high density materials presented in this work, molybdenum also found military applications, in particular as shaped charge liner in warheads [129]. For this reason, some studies on the dynamic properties of molybdenum exist. Pure molybdenum in form of rods has a young modulus and yield strength ( $0.2 \%$ def.) at room temperature of $340 \mathrm{GPa}$ and $600 \mathrm{MPa}$ respectively. Tensile strength at room temperature is 700 $\mathrm{MPa}$. It is therefore much less ductile than tantalum but less brittle than iridium or tungsten. In contrast to other metals, the ductility of molybdenum materials increases with increasing cold working [130]. Molybdenum has a DBTT which can be above room temperature since is sensitive to many factors such as impurity content, microstructure and strain rate. Different studies have assessed the dynamic response of pure molybdenum at different strain rates. In ref. [131], tests were performed in sintered, vacuum annealed, molybdenum at moderate strain rates 
(up to $100 \mathrm{~s}^{-1}$ ) and temperatures up to $330{ }^{\circ} \mathrm{C}$. Chen et al. extended this work testing molybdenum samples with various compositions, manufacturing sources, and pre-strain in a range of temperatures from $-196{ }^{\circ} \mathrm{C}$ to $1000{ }^{\circ} \mathrm{C}$ [132]. In this study, constitutive models such as J-C, Z-A and (MTS) were also extracted from the experimental data. More recently, in ref. [133], Chen et al. investigated the hot deformation behavior of molybdenum at different temperatures ranging from 1100 to $1400{ }^{\circ} \mathrm{C}$. Concerning the spall strength of molybdenum, in ref. [134] strength measurements on molybdenum in various states, including poly and single crystals, are presented. This study used high-power proton beams using the Karlsruhe Light Ion Facility (KALIF) [135]. A spall strength $1.3 \mathrm{GPa}$ at $10^{4} s^{-1}$ was reported for polycrystalline molybdenum.

TZM is a dispersion strengthened Mo alloy deriving its high strength from finely dispersed titanium and zirconium carbide particles. This alloy is $99.4 \% \mathrm{Mo}$, $0.5 \% \mathrm{Ti}, 0.08 \% \mathrm{Zr}$ and $0.01-0.04 \% \mathrm{C}$. The dispersed carbides improve significantly the TZM behavior at high temperature, inhibiting grain growth effects and increasing the recrystallization temperature up to $1400{ }^{\circ} \mathrm{C}$ [136]. In addition, yield strength is increased up to $780 \mathrm{MPa}$ at room temperature and stays above $400 \mathrm{MPa}$ even at $1100{ }^{\circ} \mathrm{C}$ [130]. It is for this high stability at high temperature that TZM is considered one of the target materials of the potential Beam Dump Facility at CERN [94]. Its experimental study in the present thesis, in addition to assess the TZM behavior at the AD-Target conditions, could be profitable for the former application, in particular if significant difference between the response of pure Mo and TZM is observed.

Regarding radiation damage on molybdenum and TZM, several studies exists since this material was also candidate for space reactors during the 70's [136] as well as candidate material for the facing-armor of Tokamak fusion reactors [137]. In ref. [138], pure Mo and TZM were irradiated by neutrons up to 19 dpa at temperatures between $425{ }^{\circ} \mathrm{C}$ and $1500{ }^{\circ} \mathrm{C}$ [138]. This study was mainly focused on void formation and swelling. In ref. [82], TZM was exposed up to $0.9 \cdot 10^{22} \mathrm{n} / \mathrm{cm}^{2}$ fast neutron fluences in the EBR-II breeder reactor at irradiation temperatures of $370-550{ }^{\circ} \mathrm{C}$. Tensile tests carried out in the irradiated TZM specimens from room temperature to $927^{\circ} \mathrm{C}$ indicated a significant lost of ductility in the material and increase of its DBTT of about $200{ }^{\circ} \mathrm{C}$. It has been also reported that this shifted rise of the DBTT can reach up to $500{ }^{\circ} \mathrm{C}$ under certain conditions [137]. More recent publications of fast neutron induced radiation damage on TZM can be found in refs. [139, 140], in which Mo and TZM were irradiated up to 13 dpa at $80-1000{ }^{\circ} \mathrm{C}$ and tested from -194 to $1000{ }^{\circ} \mathrm{C}$. Studies on proton irradiation induced damage in TZM and Mo can be found in ref. [141], achieving 0.5 dpa by a 600 $\mathrm{MeV}$ proton irradiation. 


\section{Chapter 2}

\section{Computational Methods for Simulating the Response of the AD-Target Core}

\subsection{Introduction}

In Chapter 1 an introduction to the characteristics and operation concerns of the AD-Target was presented. In this context, it was identified by means of analysis of the bibliographical information, that one of the main concerns which limits the target life and influences the $\bar{p}$ production yield is the thermo-mechanical response and stress waves produced in the target core as a consequence of the sudden deposition of energy by the primary proton beam. It is for this reason that the study of the dynamic response of the target material when subjected to sudden energy deposition is fundamental in order to reduce the uncertainties concerning the target core structural state and to provide a robust design. Any significant damage and effective loss of density of the target material will lead to target exchange, since the proton-target interactions and antiproton production will be reduced to the point that the target has to be replaced periodically to maintain the desired performance $[42,23]$. Unfortunately, post examination of failed targets is complex due to high activation of the irradiated material which implies performing the target opening in hot cell facilities using robotic hands and cutting tools. For this reason, a reliable methodology based on advanced computational tools and experimental tests is the only way to reduce uncertainties on the target response and to assess the choice of future design and candidate material. In the present 
chapter, the physics and tools employed for the numerical part of this approach are shown.

The thermo-mechanical effects induced in materials impacted by high energy particle beams have been extensively studied in accelerator technology in a wide number of facilities around the world for the design of BIDs (beam intercepting devices) such as targets, collimators and dumps. References [142, 143, 144, 145] are a few among many examples. In these studies, the energy deposition map arising as consequence of the proton beam interaction with matter is calculated by means of Monte Carlo simulations and then applied as an internal energy load to thermomechanical Finite Element (FE) solvers. The thermo-mechanical effects induced in solids were also studied analytically in ref. [146], where dynamic stresses were solved for a single traversal section of a cylinder assuming impact of a Gaussian beam. However, all these calculations are normally performed under assumptions of material elastic regime and considering quasi-static analyses, focused on steady state conditions or the design of the BIDs cooling systems since the adiabatic rise of temperatures provoked by the primary beam impact is within the order of hundreds of degrees per pulse (in the worst-case scenario). This is not the case of the AD-Target, in which the rapid heating of its core leads to a purely dynamic response, governed by stress wave phenomena and a non-linear behavior of the materials since they are loaded above its elastic region.

For these reasons, this chapter will initially delve into the principles of stress waves and vibration phenomena to establish the background necessary for describing the computational tools applied to the AD-Target core and the interpretation of the results. Then, it will present preliminary resolutions of the AD-Target core conditions using conventional Finite Element (FE) simulations. These preliminary calculations give an idea of the temperatures and stresses reached in the core of the target but, as already mentioned, this kind of approach is limited for this specific case. Therefore, a more advanced and specific numerical tool, called hydrocode, is introduced to overcome these limitations. A detailed description of these codes and the material models required to implement them are included. Finally, this chapter presents the computational domains, geometries and configurations of the hydrocodes models of the AD-Target studied in this thesis. 


\subsection{Principles of Stress Wave Propagation in Solids and Vibration Theory}

\subsubsection{Stress Wave Propagation in Solids}

Before going through the details of the computational models used to solve the dynamic conditions reached in the AD-Target core, it is important to introduce some concepts regarding stress wave propagation in solids, as some relevant aspects of the material models, such as the Equation of State (EOS), are directly related to them.

A stress (or strain) wave is a consequence of a rapidly changing load in a region of a body, and the fact that these stresses are not instantaneously transmitted from the loaded region to the rest. This leads to the situation where one portion of the body is stressed while the other portion has not experienced this stress yet. Hence, stresses and strain are transferred from the loaded regions to the unloaded at a certain velocity. This can be seen at the atomistic level as a succession of impacts between adjacent atoms, and the corresponding transmission of momentum to its neighbours. The mass, separation, and electromagnetic forces between atoms determine the way in which the stress pulse travels from one point to the other. From the point of view of continuous mechanics, these inter-atomic motions can be seen macroscopically as the displacement of a portion of the body from its original position and the transmission of this displacement to the adjacent material. Similarly, the result of these atomic electromagnetic forces that govern this motion can be translated to macroscopic material laws and properties.

Depending on the nature and magnitude of the traveling displacements and stresses, three different types of waves exist:

I The elastic waves correspond to the regime in which Hooke's principle law applies. Forces and displacement are directly proportional and no permanent deformation remains in the material when it is unloaded.

II The plastic waves, on the other hand, appear when the amplitude of the wave exceeds the elastic limit of the material. Hooke's law of linear relation between deformation and stresses does not hold anymore and permanent deformations are present.

III Finally, shock waves correspond to a type of waves in which the amplitude of stresses and displacements is that high that a traveling front of discontinuity in pressure, temperature and density is present.

In the next subsections some more details and formalisms of this sort of waves are presented. It is important to remark at this point that the type of waves present in 


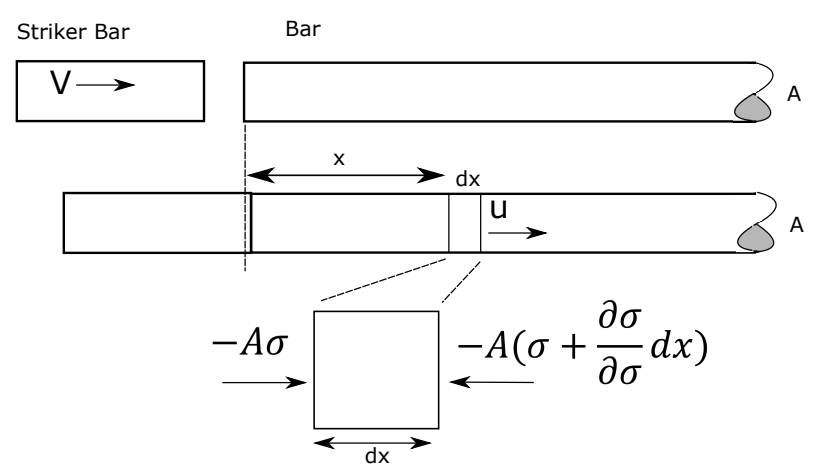

Figure 2.1: Propagation of a longitudinal elastic wave through a bar impacted by a striker.

the AD-Target and studied in detail in this thesis are just elastic and plastic. The conditions required for the formation of a shock wave (several tens of gigapascals of pressure and the presence of a discontinuous front of pressure and density), are not reached in the AD-Target. Nevertheless, some physics of shock waves are introduced here since, as it will be seen in the section 2.6, the working principles of hydrocodes and their employed material models are directly linked to them.

\section{Elastic Waves}

A very simple example, often present in the literature to instruct about the propagation of elastic waves, corresponds to the case of a disturbance traveling longitudinally along a cylindrical bar that has been impacted by a striker in one its extremities, as shown in figure 2.1. For this ideal case, stress and inertia along the transverse direction is neglected and only a displacement $u$ of the particles in the bar in the longitudinal direction is possible. If one considers, at a given time, the infinitesimal volume present between two sections of the bar, being the first section at position $\mathrm{x}$ and the second at $x+\mathrm{d} x$, and applies the second Newton's law (momentum conservation) for this infinitesimal volume:

$$
\begin{array}{r}
F=m a \\
-\left[A \sigma-A\left(\sigma+\frac{\partial \sigma}{\partial x}\right)\right]=A \rho d x \frac{\partial^{2} u}{\partial t^{2}} \\
\frac{\partial \sigma}{\partial x}=\rho \frac{\partial^{2} u}{\partial t^{2}}
\end{array}
$$

Where $u$ is the displacement of the section at position $x, A$ is area of the section of the bar, $\sigma$ and $\epsilon$ are the stress and strain, and $\rho$ is the density. Then, assuming 
that the deformation is elastic, Hooke's laws applies $(\sigma=E \epsilon)$, and $\epsilon=\partial u / \partial x$, the eq. 2.1 can be expressed as:

$$
\frac{\partial}{\partial x}\left[E \frac{\partial u}{\partial x}\right]=\rho \frac{\partial^{2} u}{\partial t^{2}}
$$

and

$$
\frac{\partial^{2} u}{\partial t^{2}}=\frac{E}{\rho} \frac{\partial^{2} u}{\partial x^{2}}
$$

Eq. 2.3 is a second order hyperbolic differential equation which has the form of the so called, wave equation. It can be shown that any type of disturbance propagating at a certain velocity in a medium which is remaining unchanged after its pass is described by this equation [147]. The term accompanying the second position derivative is the square of the propagation velocity. Thus, the velocity of propagation of the longitudinal perturbation presented here corresponds to:

$$
C_{0}=\sqrt{\frac{E}{\rho}}
$$

There are also different types of elastic waves that can propagate in solids, depending on how the motion of the particles of the solid is related to the direction of propagation of the waves themselves, and on the boundary conditions. The example just presented correspond to the so called, longitudinal or dilatational wave, in which the motion of the particles is along the direction of wave propagation. It can be shown, that the extension of the presented example to a 3-dimension continuum (no presented here for simplicity), considering tri-axial stress and an unbounded medium, leads to a similar wave equation as the one presented in 2.3, in which the velocity of propagation is:

$$
C_{0}=\sqrt{\frac{(1-\nu) E}{\rho(1+\nu)(1-2 \nu)}}
$$

Where $\nu$ is the Poisson's ratio. This is the often called "bulk" sound of speed. One can realize that the velocity just presented will be the same as the one introduced in eq. 2.4 when assuming $\nu=0$, as the shear stress was neglected in the first expression.

Other common types of elastic waves include shear waves, if which the motion of the particles is perpendicular to the direction of propagation, or Rayleigh waves 
(also known as surface waves) which are restricted to the region adjacent to the surface of a medium. All of these waves travel slower than the longitudinal waves.

\section{Plastic Waves}

As already introduced, when the propagating stresses are above the elastic limit of a ductile material, permanent plastic deformation occurs and the linear relation between stresses and deformation (Hooke's law) does not hold anymore. This has a direct influence in the wave propagation velocity and transition phenomena. As a matter of fact, when the amplitude of a pulse load exceeds the elastic limit of the material, this pulse will decompose into and elastic and plastic wave, traveling at different velocities. For example, it is shown in ref. [147] that the velocity of propagation of a plastic wave in a wire (under uni-axial stress assumption) follows the relation of eq. 2.6:

$$
C_{p}=\sqrt{\frac{d \sigma / d \epsilon}{\rho}}
$$

Where $d \sigma / d \epsilon$ is the slope of the plastic region of the stress-strain curve. Therefore, the presented velocity $C_{p}$ is only constant at a given strain. It can be seen as well that, due to yielding of the material after the elastic region, $d \sigma / d \epsilon$ will be, in principle, lower than the elastic modulus $E$. This can be seen figure 2.2-(a), where a typical stress-strain curve for ductile material is shown. Hence, the plastic waves are, in general, slower than the elastic.

However, there are extreme cases when the amplitude of the plastic wave and strain rate is high enough that the previous statement is not necessarily true. These cases are related to the formation of shock waves and take place when the medium being loaded is constrained in the laterals, whether because is physically bound, or due to the large amount of unloaded material in the surroundings which, due to high-rate phenomena, does not have time to deform laterally. In such scenarios, no strains perpendicular to the stress-wave propagation direction are allowed, hence, the state of uni-axial stress is transformed into uni-axial strain. This shift to uni-axial strain response (from the commonly known stress-strain response under uni-axial stress) has a radical impact on the stress-strain curve of the material as shown in figure 2.2-(b). This curve includes the transitions from elastic, elastic-plastic and shock regimes, and is also known as Hugoniot curve. The formal derivation behind this curve transformation is not presented here for simplicity, the reader is referred to refs. [147, 148] for more details. Instead, a description of the implications of the Hugoniot curve is included in the next paragraphs. 


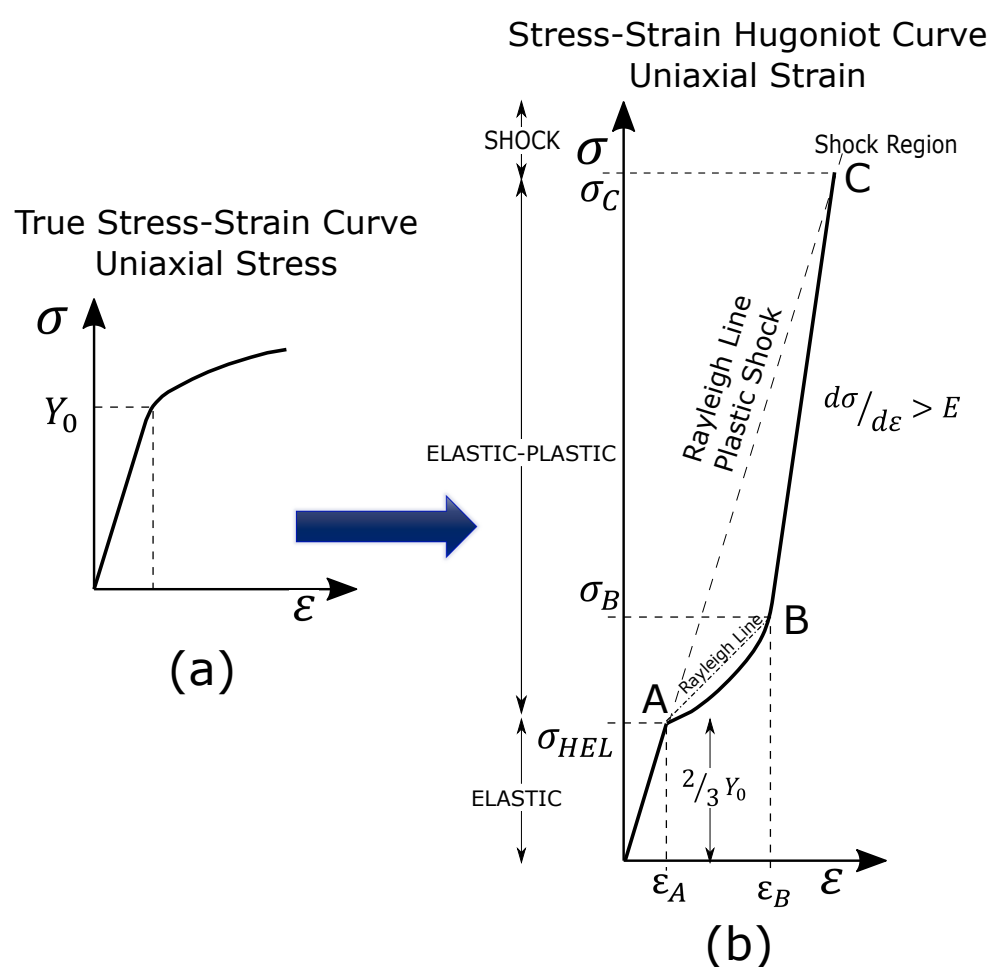

Figure 2.2: Change of the stress-strain curve when shifting from uni-axial stress (left) to uni-axial strain (right). The uni-axial strain curve extended to high pressures shown at the right is known as Hugoniot curve and is fundamental for understanding the generation of a shock wave.

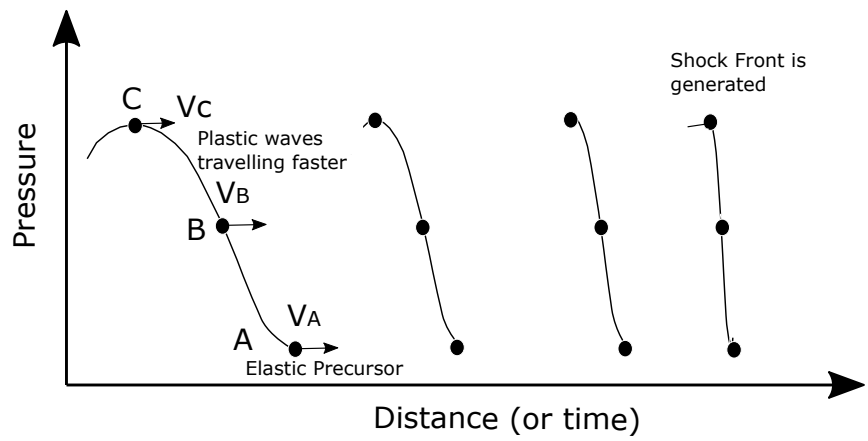

Figure 2.3: Diagram representing the formation of a shock front from the conditions in which propagating strains are that high that $d \sigma / d \epsilon>E$ (sector B-C in the Hugoniot curve shown in 2.2-(b)). The plastic waves travel then faster than the "elastic precursor", leading to the formation of a discontinuous front of pressure, i.e, a shock wave. 
As can be observed in figure 2.2-(b) the strain-stress curve for uni-axial strain has three main regions:

i If the stresses are lower than the so called Hugoniot elastic limit $\left(\sigma_{H E L}\right)$, a single elastic wave will propagate though the material. This limit is equivalent to $2 / 3 Y_{0}$ where $Y_{0}$ is the yield strength [148].

ii If the applied stress exceeds $\sigma_{H E L}$, the pulse will decompose in two types of waves; (i) an elastic one traveling with the speed already presented in eq. 2.5 (generally called "elastic precursor" since it travels faster), (ii) followed by plastic waves moving with different speeds that are function of the slope of the curve $d \sigma / d \epsilon$ at a given value of strain. This phenomenon takes place within the points A-C of the Hugoinot curve shown in figure 2.2-(b). This last statement has very strong implications, as leads to the fact that there can be a multiplicity of plastic waves, traveling with different speeds as a function of the strain. In addition, it can be observed that in the region A-C, the Hugoinot curve is concave, i.e., the slope of $d \sigma / d \epsilon$ increases with the plastic strain. This is due to the fact that the material starts behaving like a fluid due to the high pressure reached and that the repulsive forces between the molecules grow non-linearly with the compressive deformation. As a matter of fact, the $\mathrm{ABC}$ curve is parallel to the hydrostatic compression curve of the material. As a direct consequence of the increase of the slope of $d \sigma / d \epsilon$, plastic wave velocities continue increasing with the stress up the point $\mathrm{B}$, above which the slope $d \sigma / d \epsilon$ is even higher than $E$. This is the critical point leading to shock wave generation since from this moment (region BC), plastic waves travel faster than the "elastic precursor", resulting in a buildup of pressure, steepen-up wave the front and, eventually, that the plastic waves reach the "elastic precursor" and both coalesce as a shock wave. This build-up of the pressure wave and shock wave generation can be seen in the representation of figure 2.3, where A, B and C correspond to the mentioned points in the Hugoinot.

iii Shock wave region takes place above the point $\mathrm{C}$ of the curve. From this point, coalescence of the elastic precursor and the plastic wave has taken place leading to the formation of a discontinuous front of pressure, traveling faster than the original elastic wave at a shock velocity $U_{s}$. In reality, this discontinuity is an idealization as the front has a finite structure due to other material effects. Shock waves are described a bit more in detail in the next subsection. 


\section{Shock Waves}

As just introduced, a shock wave is defined by a front of discontinuity in pressure, temperature and density, traveling at a certain velocity. Shock waves are created from plastic waves if the reached stresses are higher than a certain limit, from which the waves in the high pressure loaded region travel faster than in the lower loaded region. A very common element of shock waves is also that, due to the high level of pressure reached, the shear stresses can be neglected in comparison to the hydrostatic components of the stress tensor. This is the equivalent of having a material without strength which behavior can be treated as the one of a fluid. From this assumption, a very simple and useful analogy often found in literature to derive the shock governing equations is presented in figure 2.4.

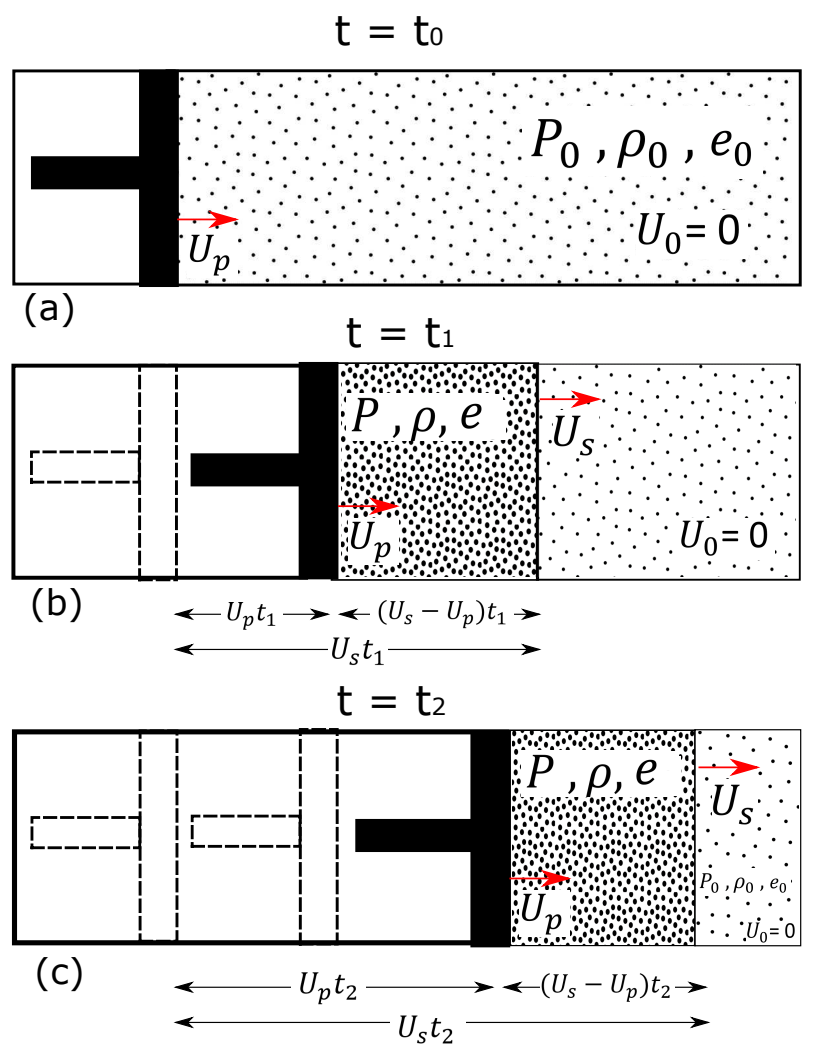

Figure 2.4: Piston analogy to understand the derivation of Rankine-Hugoinot equations. A piston traveling at a speed $U_{p}$ suddenly compresses a gas, which compression front travels at the speed $U_{s}$. 
This analogy consists in a cylinder filled by a fluid at a certain rest conditions, defined as initial pressure $P_{0}$, initial density $\rho_{0}$, and initial specific energy $e_{0}$. A piston then suddenly starts compressing the fluid at a velocity $U_{p}$. As a consequence, a disturbance front propagates ahead the piston at a velocity $U_{s}$. Hence, the fluid inside the cylinder is divided into two regions as shown in figure 2.4-(a) and (b); (i) The compressed region in which the particles move at a velocity $U_{p}$, and (ii) the uncompressed or "stationary" region, in which the particles are at rest as the disturbance has not reached them yet. Between these two regions, the propagation front moves at a velocity $U_{s}$, which is always larger than $U_{p}$. At time $t_{1}$, the piston has traveled a distance $U_{p} t_{1}$, while the shock front a distance $U_{s} t_{1}$. Therefore, mass conservation equation in the compressed region is:

$$
\begin{array}{r}
U_{s} t_{1} \rho_{0}=\rho\left(U_{s}-U_{p}\right) t_{1} \\
U_{s} \rho_{0}=\rho\left(U_{s}-U_{p}\right)
\end{array}
$$

The momentum conservation forces that, at every given time, the product of the mass and the velocity of the compressed volume is equal to the impulse given to that volume.

$$
\begin{array}{r}
\rho\left(U_{s}-U_{p}\right) t U_{p}-0=\left(P-P_{0}\right) t \\
\rho\left(U_{s}-U_{p}\right) U_{p}=\left(P-P_{0}\right)
\end{array}
$$

Finally, the energy conservation equation implies that the work done by the external forces $\left(P U_{p} t\right)$ is equal to the change in internal specific energy $\left(e_{1}-e_{0}\right) \rho_{0} U_{s} t$ plus the change in kinetic energy in the fluid $1 / 2 \rho U_{s} t U_{p}{ }^{2}$. Therefore:

$$
P U_{p}=\left(e_{1}-e_{0}\right) \rho_{0} U_{s}+\frac{1}{2} \rho U_{s} U_{p}^{2}
$$

Equations 2.7-2.9 are the so called, Rankine-Hugoniot equations, which express the relationship between state variables in a material in which a pressure discontinuity propagates. These equations can be extended to any solid subjected to shock loading under the following assumptions: (i) The shock front is discontinuity and has no apparent thickness. (ii) Shear modulus of the material is assumed to be zero. (iii) Body forces and heat conduction are negligible. (iv) There is no elastoplastic behavior and material does not undergo phase transformations. It can be also shown that the energy equation 2.9 can be also expressed in terms of pressure and specific volume from a combination with the conservation of mass and momentum, as presented in eq. 2.10 [148]. The energy equation presented in this form is known as the Hugoinot relationship, which express the locus of all shocked states of a material. 


$$
e_{1}-e_{0}=\frac{1}{2}\left(v_{0}-v\right)\left(P_{0}-P\right)
$$

Where $v$ is the specific volume $(1 / \rho)$. It can be observed then that the equations 2.7, 2.8 and 2.10 form a system of 3 equations and 5 variables $\left(P, U_{p}, U_{s}\right.$, $v$ or $\rho$, and $e$ ). An additional equation is therefore necessary to express all these variables as a function of one of them. This additional equation comes from an experimental relation between the particle velocity and shock velocity which is intrinsic to any material. Empirically, it was found that this relation can follow a polynomial relationship as shown in eq 2.11 .

$$
U_{s}=C_{0}+S_{1} U_{p}+S_{2} U_{p}^{2}+\ldots
$$

This equation is the so called Equation of State (EOS), which will be described much more in details in section 2.6.2. The EOS expresses the relation between thermodynamic state variables such as pressure, specific energy and density. As it will be seen, the EOS can be defined in many forms. Nevertheless, it is very interesting to note that it can be defined in terms of shock velocity relations, since, as a matter of fact, this relation between pressure, internal energy and density is what is governing the propagation of a wave. Indeed, the EOS states defines the rise of internal energy or pressure in a portion of material when loaded, and how this portion expands and affects the adjacent material.

\subsubsection{Free Vibrations Modes of a Cylinder}

In addition to the fundamentals of stress wave propagation recently described, it has been considered important to describe some relevant aspects about vibration theory since it will be useful to interpret many of the results of this work.

In the previous section, it was introduced the generation and propagation of elastic waves, described as the transmission of a force or displacement through a material at a given velocity. Vibration theory, on the other hand, leads with the resulting oscillatory motions in a body with a limited geometry, which are taking place as a consequence of propagation and bouncing of these elastic waves through it. There are two general types of vibration; (i) Forced vibration and (ii) Free vibration. In the former, a periodic excitation drives these oscillatory motions, while in the latter, a single excitation is necessary to excite the motion that will be just function of the mass a stiffness distribution of the body, which can be seen as a dynamical system. The descriptions presented here are limited to free vibration since in the device studied in this thesis, the load (each beam impact) can be considered as a single excitation. This is due to the fact that the repetition rate between consecutive pulses (several seconds) is very large in comparison with the 
range of time during which the excited dynamic response prevails (in the order of micro/milliseconds). Therefore, the response is well damped before the next pulse impact, disregarding the appearance of a forced vibration.

Then, in this context of free vibration, a normal mode (or natural frequency) is defined as a given state of mechanical excitation of a body, arising from the mentioned wave propagation, in which all the particles of the system are affected sinusoidally under a fixed frequency.

In general, every motion of a system responds to a superposition of many of its normal modes (under a linear-system assumption). Formally speaking, a given continuous body has almost infinite normal modes (as many as the sum of all the degrees of freedom of the atoms that form it). However, in practice, these individual motions are so constrained that the relevant modes are only function of macroscopic properties such as the stiffness, mass and geometry of the body (including its boundary conditions). Thus, any load such as a mechanical impact, can excite many of these modes. However, in some particular cases linked to a specific excitation (with a given location/direction/time duration), it is possible that the overall response is dominated only by a few modes of certain frequencies. This is the case of study of this thesis, in which the load does not come from an external impact (as in most of the vibrations problems of engineering) but from the internal expansion of the material suddenly heated by the proton beam. In that context, and taking into consideration the cylindrical shape of the target core, it will be seen that three main modes can dominate the dynamic response of the target core; radial, longitudinal and bending. Under certain assumptions, the frequency associated to these modes can be derived analytically. Some of these analytical expressions are given in the next paragraphs.

\section{Radial Modes}

The radial mode (or radial natural frequency) in a slender cylinder corresponds to the motion of particles in the radial direction. In this type of mode, all the particles along the radius move in phase inside the transverse plane of the cylinder, leading to an overall response in which the cylinder "swells" and "shrinks" radially. This mode is also known as "planar radial vibration" in the literature. In ref. [149, $150]$ is shown that, under the assumption of elastic material, zero shear stress and strain, and uncoupling the longitudinal and radial vibration, the period of such radial modes can be expressed by equation:

$$
T_{r 0}=\frac{2 \pi r}{X} \sqrt{\frac{\rho(1+\nu)(1-2 \nu)}{E(1-\nu)}}
$$


Where $r$ is the radius of the cylinder and $X$ is equal to $K_{r} r$, where $K_{r}$ is called the equivalent radial wavenumber. In ref. [150] it is also shown that $X$ can calculated by solving the following transcendental equation:

$$
X J_{0}(X)(1-\nu)-J_{1}(X)(1-2 \nu)=0
$$

Where $J_{0}$ and $J_{1}$ are Bessel functions of the first kind for order 0 and 1 respectively. Solving numerically eq. 2.13 and introducing the value of $X$ in eq. 2.12, one can obtain predictions of the expected radial vibration periods for the materials and geometries studied in this thesis. These radial periods are presented in table 2.1. Through the present thesis, this radial vibration or mode is usually just denominated "radial wave" for simplicity and to be consistent with the nomenclature used in other publications treating this phenomenon in beam intercepting devices [22, $46,146,151]$. It shall be taken into account that, strictly speaking, is not a pure wave from the wave definition in which a load is traveling from a localized area, to other unloaded areas. Instead, this radial mode is the result of infinitesimal waves with infinitesimal departing points along the radius of the cylinder, which interact in each single point. It will be shown that radial vibration modes (or waves) with similar periods as the ones presented in table 2.1 are obtained within this work, either in the numerical simulations of Chapter 3 and in the experimental data recorded in Chapter 5, even though the materials in such cases were partially subjected to plastic deformations, which are not considered by the eq. 2.12.

Table 2.1: Period of the radial mode (also denominated "radial wave" in this work) according to eq. 2.12 for the different materials of interest and rod geometries; $3 \mathrm{~mm}$ diameter for the case of the AD-Target core and $8 \mathrm{~mm}$ diameter for the rods used in the experiment described in Chapter 4.

\begin{tabular}{lcc}
\hline \hline Material & $1.5 \mathrm{~mm}$ Radius & $4 \mathrm{~mm}$ Radius \\
\hline \hline Iridium & $0.84 \mu \mathrm{s}$ & $2.25 \mu \mathrm{s}$ \\
\hline Tungsten & $0.87 \mu \mathrm{s}$ & $2.32 \mu \mathrm{s}$ \\
\hline Tantalum & $1.04 \mu \mathrm{s}$ & $2.78 \mu \mathrm{s}$ \\
\hline Molybdenum & $0.58 \mu \mathrm{s}$ & $1.71 \mu \mathrm{s}$ \\
\hline TZM & $0.67 \mu \mathrm{s}$ & $1.80 \mu \mathrm{s}$ \\
\hline
\end{tabular}




\section{Longitudinal Modes}

Similarly as the radial modes, the longitudinal extensional mode corresponds to the motion in which all the particles move in phase within the axis direction of the cylinder. It can be shown that the period associated to this mode, under the assumption of a slender cylinder, elastic behavior, and neglecting shear stress and radial coupling, is defined by:

$$
T_{l 0}=2 L \sqrt{\frac{\rho}{E}}
$$

Where $L$ is the target length. It is interesting to note the similarity of this equation with the one already presented in eq. 2.4 of the section introducing elastic waves, showing the velocity of propagation of a longitudinal wave through an uni-axial cylinder. Indeed, the period presented in eq. 2.14 is just two times the length of the cylinder $(L)$ divided by the velocity of propagation of the longitudinal elastic wave.

As with the radial vibration mode, this longitudinal vibration mode is usually just denominated "longitudinal wave" through the present thesis. Table 2.2 shows the obtained longitudinal vibration periods for the different materials and rod geometries which will be studied. Similarly as with the radial wave, it will be shown that the longitudinal period observed, either in the numerical simulations of Chapter 3 and in the experimental data recorded in Chapter 5 are very close to the ones presented in the table.

Table 2.2: Period of the longitudinal mode (also denominated "longitudinal wave" in this work) according to eq. 2.14 for the different materials of interest and rod geometries; $55 \mathrm{~mm}$ length for the case of the AD-Target core and 140-240 $\mathrm{mm}$ length for the rods used in the experiment described in Chapter 4.

\begin{tabular}{lccccc}
\hline \hline Material & Length: & $55 \mathrm{~mm}$ & $140 \mathrm{~mm}$ & $160 \mathrm{~mm}$ & $240 \mathrm{~mm}$ \\
\hline \hline Iridium & $22.7 \mu \mathrm{s}$ & $57.8 \mu \mathrm{s}$ & - & - \\
\hline Tungsten & $24.2 \mu \mathrm{s}$ & $61.5 \mu \mathrm{s}$ & - & - \\
\hline Tantalum & $32.9 \mu \mathrm{s}$ & - & $95.6 \mu \mathrm{s}$ & - \\
\hline Molybdenum & $19.3 \mu \mathrm{s}$ & - & - & $84.0 \mu \mathrm{s}$ \\
\hline TZM & $20.2 \mu \mathrm{s}$ & - & - & $88.3 \mu \mathrm{s}$ \\
\hline
\end{tabular}




\section{Bending Modes}

Finally, the other relevant modes for the application of this study are the bending or flexural modes of a cylinder. These modes are associated to the bending vibrations excited when a non-symmetric load is applied. As it will be seen in Chapter 5, this can be the case when the proton beam impacts the target cylinder with a certain off-axis, even if it is very small. There are many types of bending modes depending on the boundary conditions and the way of clamping the rod. In a general form, the periods associated to these bending modes in a slender cylinder, for an elastic material, and under Euler-Bernoulli beam theory assumption, are defined by:

$$
T_{b i}=\frac{2 \pi L^{2}}{k_{i}^{2}} \sqrt{\frac{\rho A}{E J}}
$$

Where $J$ is the area moment of inertia of the cross section of the rod and $k_{i}$ is a dimensionless parameter given by the boundary conditions (the way the cylinder is clamped). There are different periodic bending mode shapes and frequencies associated to each certain type of boundary condition, expressed by different $i$ indexes of $k_{i}$. For example, the $k_{i}$ parameter associated to the fundamental bending mode $i=1$ (the lowest frequency one) for an un-clamped cylinder (free in its extremities) is $k_{1}=4.73$ [152]. Table 2.3 shows the estimated periods of the fundamental bending mode of a free cylinder for the different materials and geometries used in the experiment of Chapters 4 and 5 . Indeed, it will be seen in Chapter 5 that, as predicted in the table, a bending wave with a period close to $1.5 \mathrm{~ms}$ was experimentally measured in a TZM target of $240 \mathrm{~mm}$ length when impacted by the proton beam.

Table 2.3: Periods of fundamental bending mode (also denominated "bending wave" in this work) according to eq. 2.15 for the different materials of interest and rod geometries used in the experiment described in Chapter 4.

\begin{tabular}{lcccc}
\hline \hline Material & Length: & $140 \mathrm{~mm}$ & $160 \mathrm{~mm}$ & $240 \mathrm{~mm}$ \\
\hline \hline Iridium & $0.57 \mathrm{~ms}$ & - & - \\
\hline Tungsten & $0.60 \mathrm{~ms}$ & - & - \\
\hline Tantalum & - & $1.07 \mathrm{~ms}$ & - \\
\hline Molybdenum & - & - & $1.42 \mathrm{~ms}$ \\
\hline TZM & - & - & $1.49 \mathrm{~ms}$ \\
\hline
\end{tabular}




\subsection{Energy Deposition in the AD-Target: FLUKA Monte-Carlo Code}

After the overview of some of the general principles of propagation of waves in solids and vibration theory presented in previous section, we proceed to focus on the application of this work, i.e, the resolution of the dynamic conditions reached in the AD-Target core. As stated in the introduction of this chapter, most of the structural and thermal numerical calculations on BIDs start from an energy deposition map arising as consequence of proton beam interaction with matter. This map is obtained by means of Monte Carlo simulations. For this thesis work, FLUKA Montecarlo code [45] is used for generating the input for all the calculations presented, both for the implicit-FE and the hydrocode simulations.

FLUKA is a multi-particle transport and interaction Monte Carlo code which is able to calculate the interaction and transport of hadrons, heavy ions, and electromagnetic particles from few $\mathrm{keV}$ (or thermal energies for neutrons) to $\mathrm{TeV}$ (such as cosmics rays) in every kind of materials.

FLUKA uses the Monte Carlo method, which is a stochastic way of solving problems through numerical simulations utilizing sequences of pseudo-random numbers. By the law of large numbers, integrals described by the expected value of some random variable can be approximated by taking the empirical mean of independent samples of the variable. The method is commonly used when the solution cannot be derived easily in any other way, such as for computational physics, physical chemistry, radiation transport, or radiation dosimetry calculations. This was invented by Stanislaw Ulam in the late 1940 and implemented by John Von Neumann in Los Alamos National Laboratory (LANL) for neutronics calculations applied to nuclear weapons [153].

The FLUKA code started being developed at CERN during the 60's. Since then, there have been four generations of the code with important upgrades, to the point in which the present version is completely different from the ones released before 1990. The current version of FLUKA includes advanced physical models able to simulate hadron inelastic nuclear interactions, elastic scatterings, nucleus-nucleus interaction, transport of charged hadrons and muons, as well as low energy neutrons, electrons, and photons [45]. The code uses microscopic models whenever possible, and keeps consistency among all the reaction steps and reaction types. Conservation laws are enforced at each step. Its physical models are continuously upgraded and benchmarked against experimental data. The code is also able to predict radiation induced damage, in terms of DPA (displacements per atoms), by estimating the number of projectile and target nucleus recoils (and subsequent subcascade of the recoils). The conversion to DPA is performed by using a modified Kinchin-Pease damage model and taking into account the effect on the displace- 


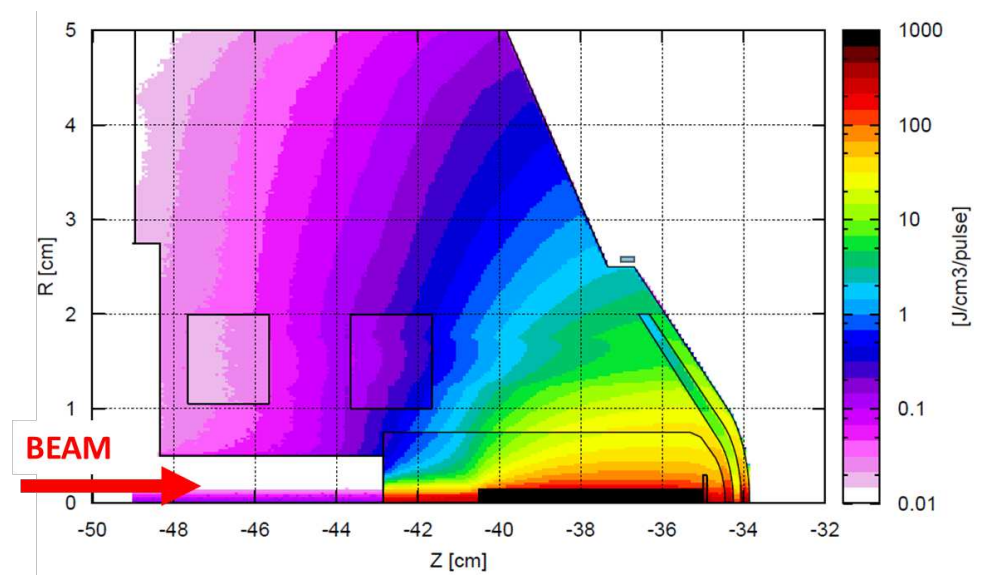

Figure 2.5: Logarithmic plot of a half-view of the deposited energy by the primary proton beam in the current design of the AD-Target obtained from FLUKA Mote Carlo Simulations (Courtesy of D. Horvath).

ment efficiency for higher recoil energies due to recombination and migration of the Frenkel pairs [154].

Figure 2.5 shows the results of FLUKA simulations of the energy deposited in the current target each time is impacted by the primary proton beam of $1.5 \cdot 10^{13} \mathrm{ppp}$. In these simulations, the computational domain is discretized in cells or bins, which size can vary from $60(\mathrm{H}) \times 60(\mathrm{~V}) \times 250(\mathrm{~L}) \mu \mathrm{m}$ in the target core (where high gradients of energy are present) to $100(\mathrm{H}) \times 100(\mathrm{~V}) \times 500(\mathrm{~L}) \mu \mathrm{m}$ at the target envelope. The simulation shows the energy deposited not only in the iridium target core, but also in its envelope, produced by the shower of particles created from the primary collisions.

From the output of these simulation, a table is created with the normalized value of the energy deposited in each bin and its position. Subsequently, these values are interpolated to the corresponding mesh of the Finite Element (FE) solvers and applied as internal power generation in the models. 


\subsection{Thermo-Mechanical Simulations using Implicit-FE ANSYS Mechanical}

In order to calculate the temperature and stresses reached in the core of the AD-Target, thermo-mechanical calculations using the commercial ANSYS $\mathbb{R}$ Mechanical [155] FE software were performed. The simulations are done in two steps:

First, a thermal-transient calculation is carried out. In this calculation, the file generated by the FLUKA simulations is maped to the ANSYS $\cap$ mesh and applied as an internal heat generation source during the 430 ns duration of the proton pulse. This interpolation and energy application process is done creating an ANSYS-table through an APDL script [156].

Once this transient thermal simulation is completed, the resulting temperature field is applied to a second transient-structural simulation. These thermal and structural simulations are therefore fully uncoupled. In any case, the application of temperature field to the structural-transient simulation is done in a stepwise mode, i.e, being consistent with the realistic and progressive increase of temperature in the target over the duration of the proton pulse impact.

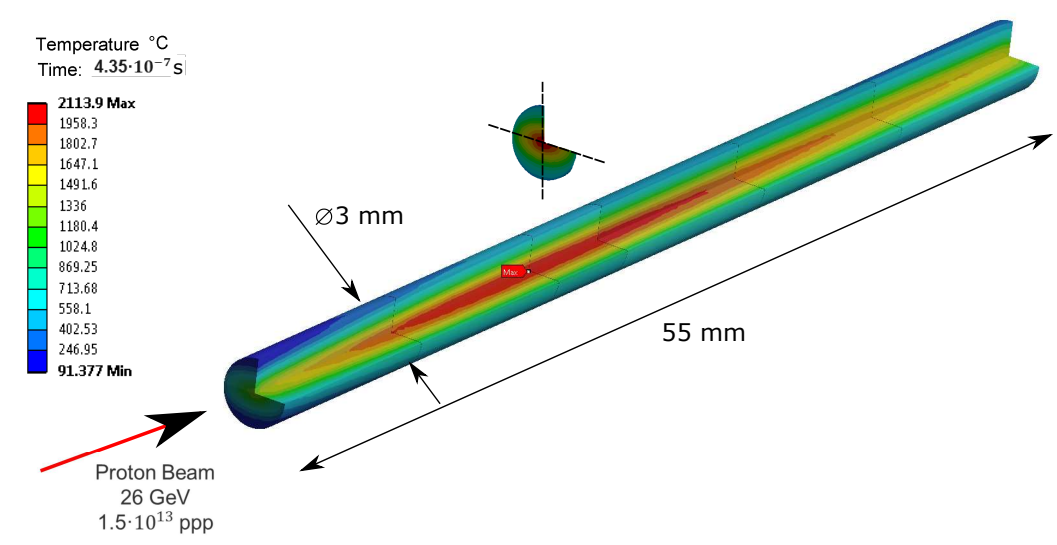

Figure 2.6: $3 / 4$ cut view of the temperature profile in the Ir target core at the end of a single $26 \mathrm{GeV}$ proton beam impact.

Figure 2.6 shows the temperature profile in a $3 / 4$ cut view of the target core at the end of a single proton pulse impact, i.e., at $430 \mathrm{~ns}$. This simulation assumes that the rod was at room temperature prior to the pulse impact. The profile shows the increase of temperature generated as a consequence of the proton-target material interaction, which reaches above $2100{ }^{\circ} \mathrm{C}$ at the center of the rod. In addition, a temperature gradient of up to $1800{ }^{\circ} \mathrm{C}$ in only $1.5 \mathrm{~mm}$ of the radial direction takes place. The expansion of the material associated to this sudden rise of temperature 
causes the vibration excitation of core and successive waves studied in detail in this thesis, in particular in Chapter 3. No thermal-boundary conditions are applied to these simulations since it is considered that during the $430 \mathrm{~ns}$ of impact there is not enough time for heat transfer processes. The iridium properties necessary for this simulation as density, specific heat and thermal conductivity are introduced as temperature-dependent variables, and obtained from the material data base MPDB [157].

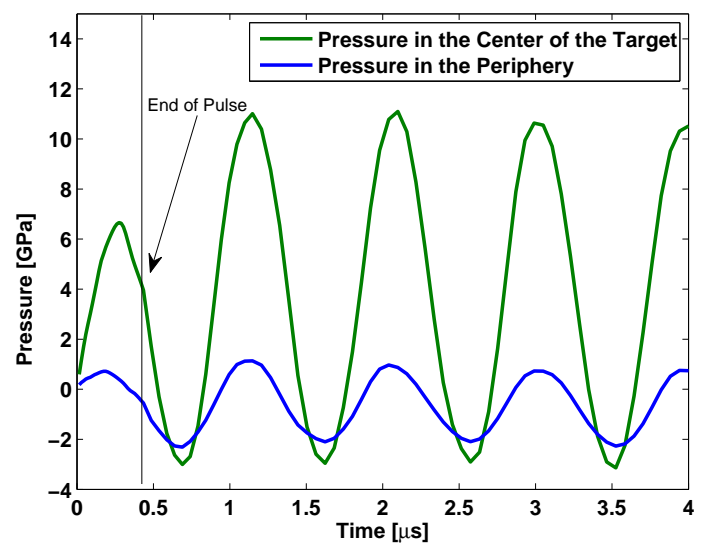

Figure 2.7: Pressure wave at the center and periphery of the $3 \mathrm{~mm}$ diameter target core obtained by transient structural calculations in ANSYS® Mechanical after applying the temperature load of figure 2.6. Note that the sign convention applied is: + compressive, - tensile. This simulation assumes that the target material behaves as perfectly elastic.

Figure 2.7 shows the pressure response in the target core center and periphery obtained from the structural-transient calculation using ANSYS mechanical after the application of the temperature load of the previous thermal transient calculation. It is important to note that, for this simulation, the material is assumed to be perfectly elastic (no yielding is considered), which is a fully unrealistic scenario. The only structural material properties added to this model are therefore the young modulus and coefficient of thermal expansion, introduced as well as temperature-dependent cariables from ref. [157]. As it can be seen in the figure, compressive pressures of several GPa's are reached in the center of the target. These high stresses are reached in an oscillating manner, arising from the excitation of a radial mode (or radial wave) with a period $0.85 \mu \mathrm{s}$, as anticipated in table 2.1. However, the result of this simulation is unrealistic since the high stresses reached will certainly make that the material experiences plastic deformation or fracture. As a matter of fact, ANSYS® Mechanical and all similar implicit solvers are limited tools for simulating this kind of processes as it is explained in detail in the next section. 


\subsection{Limitations of Implicit-FE ANSYS® Mechanical for Simulating the AD-Target Dynamic Response}

In the previous section it has been shown the preliminary results of a structural simulation using the conventional implicit FE solver of ANSYS@ Mechanical. There are several reasons why this is not the most convenient tool to solve this purely dynamic problem. These reasons are summarized in the next three subsections and will lead to the requirement of using hydrocodes.

\subsubsection{Implicit vs Explicit}

ANSYS® Mechanical uses an implicit time integration method, which is more suitable for calculations of static and quasi-static conditions. This is mainly due to the fact that the equation solved by the code is the second Newton's law, in which equilibrium is forced at every time step. This can be seen better in eq. 2.16, in which $u$ is the displacement (in the case of a thermally loaded problem, given by the rise of temperature and thermal expansion coefficient), $[\mathrm{K}]$ is the stiffness matrix, and $\mathrm{F}$ are the resultant stresses. At any given time, $\mathrm{t}$, these equations can be thought of as a set of "static" equilibrium equations. Inertia forces can be taken into account by the addition of a mass term $[\mathrm{M}]$ and the damping forces by a damping term $[\mathrm{C}]$.

$$
[M] \ddot{u}+[C] \dot{u}+[K] u=F
$$

An implicit calculation such as the one used by ANSYS@ Mechanical employs a backwards difference scheme in time. This means that the velocities and accelerations at the solution time $n+1\left(\ddot{u}_{n+1}\right.$ and $\left.\dot{u}_{n+1}\right)$ can be expressed as a function of the accelerations, velocities and displacements at the previous time step $\ddot{u}_{n}$, $\dot{u}_{n}, u_{n}$ (which are know) and the displacement at the current solution time $u_{n+1}$ (unknown). By introducing these expressions in eq. 2.16 only $u_{n+1}$ is unknown and can be obtained. However, it can be seen that an inversion of the common factor of $u_{n+1}$ in the equation (composed by a combination of [M], [C] and [K] matrices) is required at each time step. This inversion is computationally expensive, becoming especially relevant in the particular case of long transient simulations in which a large amount of time steps are required. To give an idea of this computational cost; the $4 \mu \mathrm{s}$ long structural transient simulation presented in figure 2.7, which model has 320000 hexahedral 1st order elements, took 20 hours running on a 12 cores machine. The implicit method has, on the other hand, the advantage that it is conditionally stable and can handle large time steps. This is why it is extensively used for static or quasi-static simulations in which not a large number of time steps are necessary. 
An explicit method, on the other hand, uses a different approach by applying a forward or central difference scheme in time. This means that the eq. 2.16 can be re-arranged in a way in which displacements and velocities of the previous time step, $\dot{u}_{n}$ and $u_{n}$ (which are known) are used, and accelerations $\ddot{u}_{n+1}$ at the current time step are the only unknown. This re-arrangement allows that only the mass matrix $[\mathrm{M}]$ must be inverted for solving the eq. 2.16, which is a fast and trivial process since under the lumped-mass formulation this matrix is diagonal by definition. The main drawback of this method is that the used time step is limited to small values to guarantee numerical stability. This makes this method only suitable for fast transients and highly dynamic calculations, in which in any case a large number of time steps is necessary to simulate the wave propagations. In addition, this method does not require equilibrium as the implicit one. This is actually a more realistic approach to solve dynamic problems in which, by definition, there is no equilibrium [158]. The diagram shown in figure 2.8 gives an idea of different engineering structural problems and the type of numerical approach (Implicit/Explicit) depending on the strain rates reached. It can be shown that the suitable method for the case of the AD-Target application is the explicit one, since strain rates are well above $10^{4} \mathrm{~s}^{-1}$ as shown in Chapter 3 .

\subsubsection{Strain Rate Dependence of the Material Response}

It is well known that the processes which occur when bodies are subjected to rapidly changing loads can differ significantly from those that occur under quasistatic situations. Strain rates influence the response of the material since all the deformation processes are based on dislocation dynamics, where time does matter. For instance, metals which normally experience plastic deformation at a given stress state exhibit a highly brittle response if the load is applied much faster, i.e. the strain-state curves of materials depend on the deformation velocity [147]. As described in the last section, ANSYS Mechanical is especially developed for static and quasi-static problems. A consequence of this is that material models which take into account strain rate dependence cannot be implemented with this tool. This is especially relevant for the case of application of the present work, in which, due to the extremely fast thermal load application $(0.4 \mu \mathrm{s})$, the material will clearly show strain rate dependence. A summary of different strain-dependent material models which can be used for this purpose is presented in section 2.6.3. 


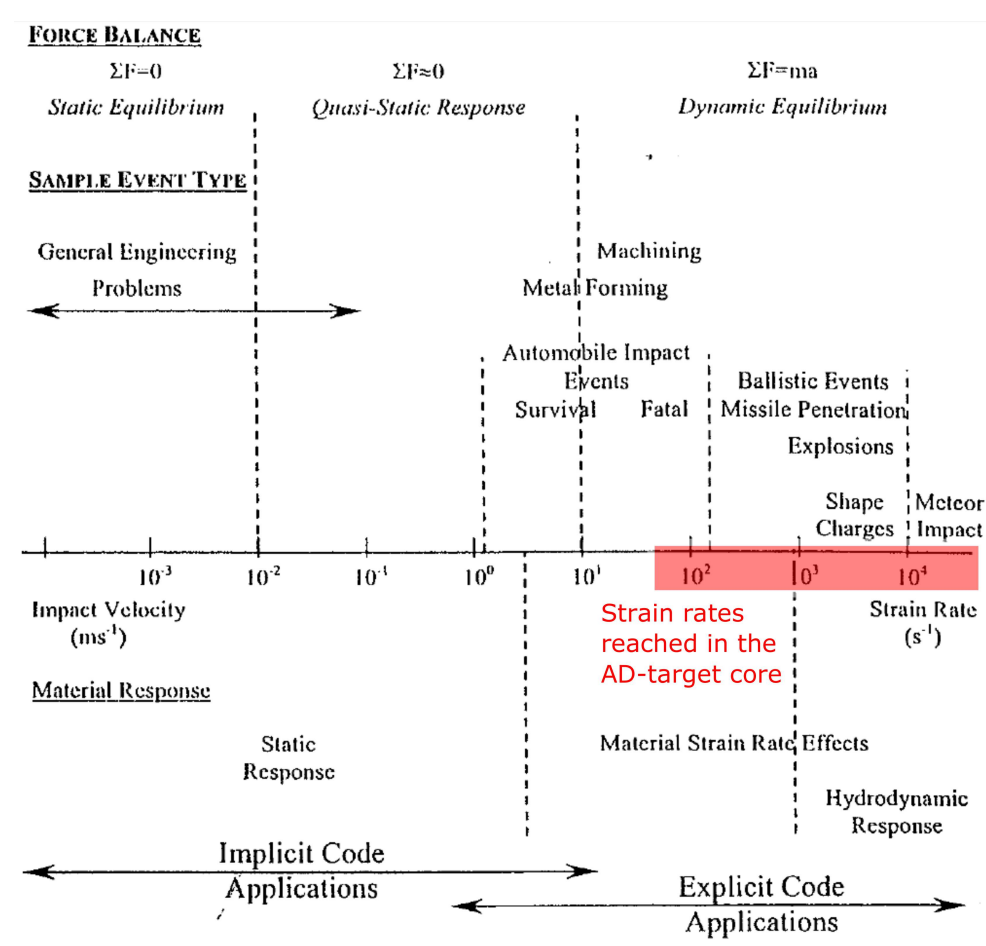

Figure 2.8: Summary of engineering structural problems and type of numerical approach (Implicit/Explicit) depending on the strain rates [158]. As shown in Chapter 3, strain rates well above $10^{4} \mathrm{~s}^{-1}$ are reached in the AD-Target core, requiring the use of explicit codes for this application.

\subsubsection{Non-linearity, Yielding and Fracture}

In addition to the computational efficiency and strain-rate material dependence, there are several other reasons that reduce the applicability of an implicit FE to the case of study. These reasons are not only related with the velocity of application of the load but with the magnitude of stresses reached. As it was shown in figure 2.7, pressures and stresses reached are well above the material yield. In order to properly simulate the material response it will be necessary to include a model that takes into account the material behavior beyond yielding. The implementation of material response above yielding can be included in ANSYS Mechanical by the definition of a bi-linear or multi-linear kinematic or isotropic hardening models [155]. However, these models are only temperature dependent (strain rate is not taken into account) and, in addition, their inclusion generates non-linear structural behavior since the stiffness matrix changes in every time step during the simulation. In this context, explicit codes also handle better nonlinearities than the implicit ones, since in the latter each step requires a series 
of trial solutions (iterations) to establish equilibrium within a defined tolerance. In an explicit analysis, no iteration is required since the nodal accelerations are solved directly. Finally, the high level of stresses may lead, in addition, to yielding, cracking or fracture of the target material. The prediction of this fracture of the target core is important in order to anticipate reduction of target effective density and therefore reduction of $\bar{p}$ production. ANSYS Mechanical does not allow the inclusion of failure models in a straight-forward manner.

\subsection{Introduction to Hydrocodes}

As justified in the previous section, conventional implicit FE codes like ANSYS Mechanical are not the most suitable tool to simulate the extreme dynamic response of the AD-Target core when subjected to proton beam impact. Luckily, computational tools which do not have the aforementioned drawbacks exist, and these are called hydrocodes.

Hydrocodes are a family of advanced, highly non-linear computational tools which are able to fully simulate the dynamic response of materials [148]. Differently from conventional structural solvers, which are solving the second Newton's law (eq. 2.16), hydrocodes numerically solve the full system of partial differential equations arising from mass, momentum and energy conservation. Eqs. 2.17, 2.18 and 2.19 show respectively these partial differential equations in a Lagrangian formulation (mesh moves and distorts with the material). The density at any time can be determined from the current volume of the zone and its initial mass. These equations are solved explicitly for each element in the model, based on input values at the end of the previous time step. Small time increments are used to ensure stability and accuracy of the solution. As introduced in the previous chapter, equilibrium is not sought in explicit dynamics. The results are simply taken from the previous time step to predict results at the next one. There is no requirement for iteration [159]. Mass conservation equation gives:

$$
\frac{\rho_{0} V_{0}}{V}=\frac{m}{V}
$$

The three components of the momentum conservation equation that must be solved are:

$$
\begin{aligned}
\rho \ddot{x} & =f_{x}+\frac{\partial \sigma_{x x}}{\partial x}+\frac{\partial \sigma_{x y}}{\partial y}+\frac{\partial \sigma_{x z}}{\partial z} \\
\rho \ddot{y} & =f_{y}+\frac{\partial \sigma_{y x}}{\partial x}+\frac{\partial \sigma_{y y}}{\partial y}+\frac{\partial \sigma_{y z}}{\partial z} \\
\rho \ddot{z} & =f_{z}+\frac{\partial \sigma_{z x}}{\partial x}+\frac{\partial \sigma_{z y}}{\partial y}+\frac{\partial \sigma_{z z}}{\partial z}
\end{aligned}
$$


Applying the energy conservation equation to an elemental volume of the solid, gives the following equation:

$\dot{e}=\frac{1}{\rho}\left(\sigma_{x x} \dot{\epsilon}_{x x}+\sigma_{y y} \dot{\epsilon}_{y y}+\sigma_{z z} \dot{\epsilon}_{z z}+2 \sigma_{x y} \dot{\epsilon}_{x y}+2 \sigma_{y z} \dot{\epsilon}_{y z}+2 \sigma_{z x} \dot{\epsilon}_{z x}\right)+f_{x} \dot{x}+f_{y} \dot{y}+f_{z} \dot{z}$

Hydrocodes are able to simulate the material response at different thermodynamic and mechanical states (including response beyond plasticity and fracture) taking into account strain rate and temperature dependence by coupling these equations with material models as equations of state (EOS), strength and failure models (which are explained in detail in sections 2.6.2, 2.6.3 and 2.6.4).

Hydrocodes were invented in Los Almos National Laboratory in the 50s. It is interesting to mention that they started being developed also by Von Neumann [160], as the Monte Carlo method used in this work and described in section 2.3. Actually, these are just two examples of a significant amount of his contributions to mathematics and computational science. The name of "hydrocodes" comes from their first applications when numerical calculations of high velocity impacts were performed assuming hydrodynamic behavior (material strength is not considered) as commonly found in shock problems, where the pressures in the colliding materials exceed their strength by several orders of magnitude [148]. Nowadays, the deviatoric behavior and strength of the material is also taken into account through material strength constitutive laws. However, they maintain their primary name.

Hydrocodes have been historically developed and used in multiple ballistic and military applications where the dynamic load originates from high velocity impacts and explosions. In addition, they have started being applied in accelerator technology for simulations of BIDs, where the load is generated by the sudden thermal expansion of the material impacted by particle pulses and the subsequent propagation of the produced stress waves. Current programs for development of accelerator technologies require increasing particle beam energies and intensities, which push engineers to use these codes to investigate mechanical damage in materials hit by high intensity beams as a consequence of abnormal operation. Simple 2-D hydrodynamic calculations were already used at CERN in 1984 to assess the response of solids and liquid materials to the impact of proton pulses [161]. In a similar way, hydrodynamic calculations for assessing the tolerance of the SSC (Superconducting Super Collider) beam dump to a hypothetical full impact of 20$\mathrm{TeV}$ proton beam was done in 1993 [162]. More recent and comprehensive analyses using the hydrocode AUTODYN $\cap$ were executed for the study of the structural behavior of the Main LHC absorber block in case of a total beam dilution failure [163]. 
Numerical studies of high dynamic transient effects of pulse beams on BIDs can be found in [151, 164], where hydrodynamical calculations for uranium beam impacted on copper targets for ISOLDE were performed. Recent publications for research on new collimator materials using ANSYS AUTODYN $R$ can be found in $[165,166]$, while using as well LS-DYNA@ in references $[167,168,169,170]$. In the same way, literature including hydrodynamic calculations using BIG2 code for the design of solid targets for the FAIR facility in Germany can be found in [171, 172], as well as for studying the penetration of LHC proton beams in carbon targets and hydrodynamic tunnelling effect [173].

The next subsections include a more detailed description of the material models employed by the hydrocodes.

\subsubsection{Stress Tensor Decomposition}

In continuum mechanics the stress state of a given element in a material is expressed by a second order tensor in which its nine components represent the stress acting on its six element sides from a given coordinate system. It is common to find in the literature a decomposition of the stress tensor in two components as described in eq. 2.20 .

$$
\left[\begin{array}{lll}
\sigma_{x x} & \sigma_{x y} & \sigma_{x z} \\
\sigma_{y x} & \sigma_{y y} & \sigma_{y z} \\
\sigma_{z x} & \sigma_{z y} & \sigma_{z z}
\end{array}\right]=\left[\begin{array}{lll}
s_{x x} & s_{x y} & s_{x z} \\
s_{y x} & s_{y y} & s_{y z} \\
s_{z x} & s_{z y} & s_{z z}
\end{array}\right]-\left[\begin{array}{ccc}
P & 0 & 0 \\
0 & P & 0 \\
0 & 0 & P
\end{array}\right]
$$

Where $P$ is the pressure, defined as the negative mean of the diagonal components of the stress tensor in eq. 2.20. Note the minus sign due to a different sign convection between stress and pressure.

$$
P=-\frac{1}{3}\left(\sigma_{x x}+\sigma_{y y}+\sigma_{z z}\right)
$$

while:

$$
\left[\begin{array}{ccc}
s_{x x} & s_{x y} & s_{x z} \\
s_{y x} & s_{y y} & s_{y z} \\
s_{z x} & s_{z y} & s_{z z}
\end{array}\right]=\left[\begin{array}{ccc}
\sigma_{x x}+P & \sigma_{x y} & \sigma_{x z} \\
\sigma_{y x} & \sigma_{y y}+P & \sigma_{y z} \\
\sigma_{z x} & \sigma_{z y} & \sigma_{z z}+P
\end{array}\right]
$$

The physical interpretation of this decomposition is that the diagonal tensor represents the hydrostatic part of the stress state while the $\overline{\bar{s}}$ tensor is the so called, deviatoric term. The hydrostatic governs the consequences of volume changes in an element of material while the deviatoric deals with its deformation or change of shape. 
It is interesting to mention that in most engineering applications, in particular the ones dealing with yielding in metals, the stress state of a material is commonly expressed by the definition of an equivalent stress, such as von Mises equivalent stress. This equivalent stress is in reality derived from the second invariant of the stress tensor of eq. 2.20 (thus independent from the coordinate system). The definition of this invariant is presented in eq. 2.23.

$$
\sigma_{V M}=\sqrt{\frac{\left(\sigma_{x x}-\sigma_{y y}\right)^{2}+\left(\sigma_{y y}-\sigma_{z z}\right)^{2}+\left(\sigma_{z z}-\sigma_{x x}\right)^{2}+6\left(\sigma_{x y}^{2}+\sigma_{y z}^{2}+\sigma_{z x}^{2}\right)}{2}}
$$

It can be easily seen that von Mises equivalent stress can be expressed in terms of the components of the deviatoric component, since $\sigma_{i i}-\sigma_{j j}=s_{i i}-s_{j j}$ and $\sigma_{i j}=s_{i j}$ for $i$ different from $j$. Therefore the eq. 2.23 stays:

$$
\sigma_{V M}=\sqrt{\frac{\left(s_{x x}-s_{y y}\right)^{2}+\left(s_{y y}-s_{z z}\right)^{2}+\left(s_{z z}-s_{x x}\right)^{2}+6\left(s_{x y}^{2}+s_{y z}^{2}+s_{z x}^{2}\right)}{2}}
$$

The von Mises stress is therefore a representation of the deviatoric stress, independent from pressure and related to change of shape in the material. In this way, the von Mises criterion suggests that yielding of materials begins when this stress invariant reaches a certain value. This value is usually obtained experimentally in standardized uniaxial tests and is extrapolated to any sort of multiaxial load which exposes the material to shear stress and deformation. The pressure, on the other hand, is a scalar that represents the hydrostatic component of the load. Therefore, by using this decomposition and looking at these two scalars, pressure and von Mises equivalent stress, it is possible to see which kind of loading is present in a material element.

Likewise, the same kind of decomposition is applied as well to the strain tensor as shown in 2.25:

$$
\left[\begin{array}{ccc}
\epsilon_{x x} & \epsilon_{x y} & \epsilon_{x z} \\
\epsilon_{y x} & \epsilon_{y y} & \epsilon_{y z} \\
\epsilon_{z x} & \epsilon_{z y} & \epsilon_{z z}
\end{array}\right]=\left[\begin{array}{lll}
\epsilon_{x x} & \epsilon_{x y} & \epsilon_{x z} \\
\epsilon_{y x} & \epsilon_{y y} & \epsilon_{y z} \\
\epsilon_{z x} & \epsilon_{z y} & \epsilon_{z z}
\end{array}\right]-\frac{1}{3}\left[\begin{array}{ccc}
\epsilon_{v} & 0 & 0 \\
0 & \epsilon_{v} & 0 \\
0 & 0 & \epsilon_{v}
\end{array}\right]
$$

Where $\epsilon_{v}$ is the volumetric strain, which is also representing the change of density in a loaded material since $\epsilon_{v}=\frac{\Delta V}{V_{0}}=\frac{\rho_{0}}{\Delta \rho}$. The equivalent strain, on the other hand, is shown in eq. 2.26 and is given by a similar expression as the equivalent stress of eq. 2.24 (only dependent on the deviatoric part), but with the components of the strain tensor and multiplied by a factor of $\sqrt{2} / 3$.

$$
\epsilon_{e q}=\frac{1}{3} \sqrt{\left(\epsilon_{x x}-\epsilon_{y y}\right)^{2}+\left(\epsilon_{y y}-e_{z z}\right)^{2}+\left(\epsilon_{z z}-\epsilon_{x x}\right)^{2}+6\left(\epsilon_{x y}^{2}+\epsilon_{y z}^{2}+\epsilon_{z x}^{2}\right)}
$$




\subsubsection{EOS - Governing the Hydrostatic Component}

The decomposition of the stress and strain tensors in hydrostatic and deviatoric terms is particularly relevant to understand how hydrocodes deal with the mass, momentum and energy equations. As it can be seen, no information about the material properties is included in eqs. 2.17-2.19 since they are just representing conservation laws. In order to fully solve these equations they have to be coupled with material laws which define the material behavior. This is done by three ingredients, principally by an Equation of State (EOS), and a Strength Model together with, in a lesser extent, a Failure Model when necessary.

As already introduced in section 2.2.1 describing shock waves, an EOS is a function which expresses the relation between thermodynamic state variables as pressure, specific energy and density as presented in eq. 2.27:

$$
P=f(\rho, e)
$$

EOS were originally developed for ideal gases and subsequently extended to all states of matter [174]. In the decomposition of the stress tensor in hydrostatic and deviatoric component presented in the previous section, the EOS governs the hydrostatic component associated with the change of pressure as a function of density and internal energy. In this way, the EOS describes the material behavior from a thermodynamics point of view. As it was already introduced in section 2.2.1 of this chapter, the EOS is strongly correlated with the shock response of a material and wave propagation velocity.

In that sense, the simplest EOS which can be considered is just the bulk modulus. This can be seen as an energy independent EOS since it expresses the relation between volumetric deformation (density) and pressure. Clearly, this is a simplistic approach. There are different EOS formulations depending on the regime of application and assumptions made. Ideally, the EOS can include a strong energy dependence and describe the material behavior in a full thermodynamic regime, even including changes of phase. However, in practice it is difficult to cover different phases with a single formulation. Some EOS formulations and their assumptions are included in the next subsections.

\section{Linear EOS}

A linear EOS assumes that the existing relation between pressure, density and internal energy is linear. As previously stated, the bulk modulus, K, can be considered as a simple, energy-independent linear EOS. Since, according to linear elasticity laws, pressure can be expressed as: 


$$
P(\rho)=K\left(\frac{\rho}{\rho_{0}}-1\right)
$$

This EOS can be extended and made energy dependent by the addition of an extra term as shown in eq 2.29. In this way, the expansion and rise of pressure in a material as a consequence of an increase of temperature (or internal energy) can be taken into account.

$$
P(\rho, e)=K\left(\frac{\rho}{\rho_{0}}-1\right)+\gamma_{0} \rho e
$$

where $\gamma_{0}$ is a non-dimensional number called the Grüneisen parameter, which is a measure of the change in pressure produced by a change in the system total specific energy under the condition of constant volume [175], i.e:

$$
\gamma=\left.v\left(\frac{\partial P}{\partial e}\right)\right|_{v}
$$

where $v$ is the specific volume $v=1 / \rho$ and $e$ is the specific energy. Due to its simplicity and linear-approximation, linear EOS can be only applied if a small density variation is involved.

\section{Linear-Polynomial EOS}

A more generalized EOS is the linear-polynomial one. This EOS is applicable to a wider range of densities and energies since pressure-density-energy relation is extended to a $3^{\text {rd }}$ order polynomial, fitted by six constants as shown in eq. 2.31:

$$
P=C_{0}+C_{1} \mu+C_{2} \mu^{2}+C_{3} \mu^{3}+\left(C_{4}+C_{5} \mu+C_{6} \mu^{2}\right) e
$$

where $\mu$ is the volumetric parameter defined as $\mu=\rho / \rho_{0}-1$. Note that the volumetric parameter is just the inverse of the volumetric strain, $\epsilon_{v}$, present in the strain tensor decomposition of eq. 2.25.

\section{Mie-Grüneisen EOS}

The Mie-Grüneisen EOS [175] is a particular case of Polynominal EOS which is often used to determine the pressure state of solids exposed to shocks. In this formulation, it is assumed that the Grüneisen parameter defined in eq. 2.30 is independent from pressure and internal energy. In this way, eq. 2.30 can be integrated with respect to a reference state giving: 


$$
P=P_{H}+\gamma \rho\left(e-e_{H}\right)
$$

where $P_{H}, e_{H}$ are pressure and specific energy in a reference state, in this case in the Hugoniot-curve which, as already introduced in section 2.2.1, describes the relationship between the possible thermodynamic states on both sides of a shock wave front [148]. Therefore, $P_{H}$ and $e_{H}$ can be defined as a function of shock jump conditions and its parameters, such as elastic sound speed $C_{0}$ and the 1st order parameter $\left(S_{1}\right)$ satisfying the well experimentally-known relation of shock front velocity $U_{s}$ and particle velocity $U p$, i.e: $U_{s}=C_{0}+S_{1} U_{p}$ [148] (already introduced in eq. 2.11). The reference states of eq. 2.32 can be therefore defined as follow:

$$
\begin{gathered}
P_{H}=\frac{\rho_{0} C_{0}^{2} \mu(1+\mu)}{\left[1-\left(S_{1}-1\right) \mu\right]^{2}} \\
e_{H}=\frac{P_{H} \mu}{2 \rho_{0}(1+\mu)}
\end{gathered}
$$

The Mie-Grüneisen EOS is therefore defined by eqs. 2.32-2.34, in which the input parameters are $C_{0}, S_{1}$ and $\gamma$. The parameters $C_{0}, S_{1}$ are obtained by dynamic and shock experiments making measurements of $U_{p}$ and $U_{s}$ and searching for the empirical linear relationship between these two variables. These values can therefore be found in the literature for several materials. The Grüneisen parameter $\gamma$, on the other hand, can be estimated from eq. 2.30 by:

$$
\gamma=v\left(\frac{\partial P}{\partial e}\right)_{v}=v \frac{\left(\frac{\partial P}{\partial T}\right)_{v}}{\left(\frac{\partial e}{\partial T}\right)_{v}}
$$

where, thanks to the triple product rule of calculus, $\left(\frac{\partial P}{\partial T}\right)_{v}$ can be expressed as:

$$
\left(\frac{\partial P}{\partial T}\right)_{v}=-\left(\frac{\partial P}{\partial v}\right)_{T}\left(\frac{\partial v}{\partial T}\right)_{P}
$$

with the result:

$$
\gamma=-v \frac{\left(\frac{\partial P}{\partial v}\right)_{T}\left(\frac{\partial v}{\partial T}\right)_{P}}{\left(\frac{\partial e}{\partial T}\right)_{v}}
$$


where:

$$
\begin{aligned}
\frac{1}{v}\left(\frac{\partial v}{\partial T}\right)_{P} & =\text { Volumetric Thermal Expansion Coefficient }(3 \alpha)^{1} \\
-v\left(\frac{\partial P}{\partial v}\right)_{T} & =K \text { Bulk Modulus } \\
\left(\frac{\partial e}{\partial T}\right)_{v} & =C_{v} \text { Specific Heat Capacity at constant volume }
\end{aligned}
$$

Therefore, the Grüneisen parameter $\gamma$ in its macroscopic formulation is equal to:

$$
\gamma=v \frac{3 \alpha K}{C_{v}}
$$

All the parameters necessary for eqs. 2.32-2.34 can be obtained experimentally. Another compact and more complete formulation of the Mie-Grüneisen EOS commonly used by the hydrocodes, is finally presented in eq. 2.40 [176]. This formulation is valid for compression and takes into account, in addition to $S_{1}$, two extra coefficients for a quadratic experimental fitting of the $U_{s}-U_{p}$ curve. Moreover, this formalization includes a first order volume correction ( $a$ parameter) of the Grüneisen parameter, which is defined at 0 pressure $\gamma_{0}$.

$$
P(\mu, e)=\frac{\rho_{0} C_{0}^{2} \mu\left[1+\left(1-\frac{\gamma_{0}}{2}\right) \mu-\frac{a}{2} \mu^{2}\right]}{\left[1-\left(S_{1}-1\right) \mu+S_{2} \frac{\mu^{2}}{\mu+1}-S_{3} \frac{\mu^{3}}{(\mu+1)^{2}}\right]^{2}}+\left(\gamma_{0}+a \mu\right)(\mu+1) \rho e
$$

While the relation of eq. 2.40 is only valid for compressing material, the expression 2.41 applies to expanded material:

$$
P(\mu, e)=\rho_{0} C_{0}^{2} \mu+\left(\gamma_{0}+a \mu\right)(\mu+1) \rho e
$$

\section{Tillotson and Puff EOS}

Tillotson EOS [177] was developed to provide an accurate description of the behaviour of metallic materials over the wide range of pressure and density in hypervelocity impacts (above the speed of sound). This EOS is able to predict, in addition to the extremely high pressure conditions taking place after a shock, changes of phase in the cases where the shock energy is sufficient to melt or vaporize the material. To do that, the Tillotson EOS divides the total range of the pressure-volume plane into four regions, covering pressures up to 1000 Mbar. However, this equation of state does not describe the material at tensile pressures,

\footnotetext{
${ }^{1}$ For isotropic material, where $\alpha$ is the linear expansion coefficient.
} 
therefore is not convenient for the case of application in this thesis, where pressures are relatively low (from the point of view of shock physics) and negative pressures (tensile) are reached as suggested by the ANSYS implicit simulations shown in figure 2.7 .

Similarly, Puff EOS was developed for the description of states of matter involved from cold to hot shock and high expansion. Like the Tillotson EOS, the plane pressure-volume is divided into three separate regions instead of four. Two of the regions correspond to whether the material is compressed or expanded and, if expanded, whether the energy is less or greater than the sublimation energy. The formulations of these regions are based on Mie-Gruneisen forms but with a variable Gruneisen $\gamma$ in the expanded phase to give the required convergence to perfect gas behavior at very large expansions, when the energy is greater than the sublimation energy [178].

\section{SESAME EOS}

SESAME is a data library containing thermodynamic properties (EOS) in a tabular form of a large number of materials [179]. The tabular formulation allows the description of the material in multi-phase regime, covering a significantly wide range of pressures and densities and including phases transitions. This is possible since the tabular EOS can be formed using various combinations of different theoretical models depending on the regime. The SESAME library has been developed since the 70s and currently contains data for about 150 materials, including (amongst others) simple elements, compounds, metals, minerals, polymers and mixtures. This library is under property of LANL and an access request must be made in order to use it [180].

\subsubsection{Strength Model - Governing the Deviatoric Component}

In the same way as the EOS governs the hydrostatic part of the tensor decomposition explained in section 2.6.1 (pressure/density relation), the strength model governs the deviatoric part of this decomposition. The strength model is therefore a constitutive relation that links deformation or change of shape of a material under a given load, i.e, links strain with stress. From this point of view, the most simplistic strength model is just the shear modulus (under the assumption of a strain rate and temperature independent, pure elastic material). A conventional strain-stress curve obtained, for example, from a standard tensile tests is as well a strength model, which can describe the material response beyond yielding (the so called, flow stress). The strain-stress relation is intrinsically the consequence of atoms and lattice dislocations in the material micro-structure. For this reason, it is easy to understand that in reality these dislocation processes are as well function of temperature, strain rate and, in some regimes, even pressure. Hence, the general 
formulation of a strength model is presented in eq. 2.42, where $\sigma$ and $\epsilon$ are the scalars associated to equivalent stress and strain (such as von Mises) introduced in eqs. 2.24 and 2.26 .

$$
\sigma=f(\epsilon, \dot{\epsilon}, T, P)
$$

There are a wide number of strength model formulations depending on assumptions in the strain rate and temperature dependence, type of material and regime of application. Some of them are empirical, semi-empirical and physically-based models. The empirical models have not any physical basis and are obtained by interpolation of the experimental data. Semi-empirical models have a physical based formulation but require as well some experimental data fitting. On the other hand, the physically-based models are obtained from microscopic dislocation phenomena and fundamental lattice properties. In the next sections some of these models are introduced.

\section{Johnson-Cook Strength Model}

Johnson-Cook (J-C) model [181] is one of the simplest temperature and strain rate dependent strength models, which takes into account the material response beyond plasticity (flow stress). It is also one of the most widely used model, due to its simplicity and large material data availability. Eq. 2.43 shows its formulation where $\epsilon_{p}$ is the plastic strain, $\epsilon_{0}$ is the reference strain, $T_{m}$ is the melting temperature, and $T_{r}$ is the reference temperature. $\mathrm{J}-\mathrm{C}$ model is a pure empirical model in which five free parameters $A, B, C, n$ and $m$ are obtained from data fitting of experimental $\sigma-\epsilon_{p}$ curves. $A$ is the yield strength, $B$ and $n$ are work hardening parameters, $C$ expresses the strain rate sensitivity and $m$ is the thermal softening coefficient. In addition, the model considers that, when the temperature is above $T_{m}$, the material melts and its mechanical strength is 0 . The experimental curves used to fit these parameters are generated by dynamic testing at different temperatures and strain rates. More details of these dynamic tests such as Hopkinson Pressure Bar Tests [182] is provided in section 2.7.3.

$$
\sigma=\left(A+B \epsilon_{p}^{n}\right)\left(1+C \ln \frac{\dot{\epsilon_{p}}}{\dot{\epsilon_{0}}}\right)\left(1-\left(\frac{T-T_{r}}{T_{m}-T_{r}}\right)^{m}\right)
$$

This model can be seen as an extension of the simple strain-hardening power law relation of Hollomon $\left(\sigma=K \epsilon^{n}\right)$ [183], but adding two additional multiplicative terms in the equation to take into account the temperature and strain rate. It is interesting to note that these terms imply scaling and not a shape modification. In addition, this model assumes that the temperature and strain rates are decoupled in the $\sigma-\epsilon_{p}$ response, i.e. the influence of the temperature at low and high strain 
rate is the same and vice versa. Furthermore, the empirical nature of the model allows in principle its use only in the regime at which its free parameters have been fitted. This model neglects the influence of pressure and changes in volume on the flow stress. Besides, it considers the melting temperature as a constant, while in reality the solid-liquid transition is influenced by pressure. These are simplifying assumptions that limit the application of this model to a restricted range of pressures and its use is highly discouraged for shock problems such as for explosions or hyper-velocity impacts. Nevertheless, regarding the application for this thesis, it has been shown to provide good agreement between experiments and simulations in other studies of proton beam impact phenomena on materials [165].

\section{Zerilli-Amstrong Strength Model}

The Zerilli-Amstrong model (Z-A) is a semi-empirical model based on simplified dislocation mechanisms [184]. As the J-C model, it takes into account the effects of strain hardening, strain-rate hardening and thermal softening. In addition, the effect of grain size has also been included in the formulation. The relation has a relatively simple expression compared to other dislocation-based constitutive models [185]. The Z-A models presents as well a different formulation for fcc (face-centered cubic) and bcc (body-centered cubic) metals, shown in eq. 2.44 and eq. 2.45 respectively.

$$
\begin{gathered}
\sigma=Y_{0}+C_{2} \sqrt{\epsilon_{p}} e^{-C_{3} T+C_{4} T \ln \epsilon_{p}}+\frac{k}{\sqrt{l}} \\
\sigma=Y_{0}+C_{1} \sqrt{\epsilon_{p}} e^{-C_{3} T+C_{4} T \ln \epsilon_{p}}+C_{5} \epsilon_{p}^{n} \frac{k}{\sqrt{l}}
\end{gathered}
$$

Where the parameters $Y_{0}, C_{1}, C_{2}, C_{3}, C_{4}, C_{5}, n$ and $k$ are obtained from experimental data while $l$ is the average grain size diameter. It can be seen that for the bcc metals the formulation adds an independent term - $C_{5} \epsilon_{p}^{n}$ - to take into account separately the plastic strain-hardening contribution to the flow stress. The Z-A model normally achieves better data predictions than the $\mathrm{J}-\mathrm{C}$ model; however, it is important to mention that this model also assumes the shear modulus and the melting temperature to be constant and pressure independent. 


\section{Steinberg-Guinan and Steinberg-Lund Strength Models}

More advanced strength models concerning material dynamic behavior are the Steinberg-Guinan (S-G) model [186] and its extension, the Steinberg-Lund (S-L) model [187]. The S-G model takes into account the temperature, strain and pressure dependence in the shear modulus and flow stress. On the other hand, the S-G model is strain-rate independent. This is justified by the assumption that beyond a certain threshold of deformation velocity, the strain-rate has no longer influence of the response. This threshold was identified above $10^{5} \mathrm{~s}^{-1}$ strain rate and $10 \mathrm{GPa}$ pressure in shock loaded experiments [186]. This is therefore a strength model suitable for shock wave problems at which high strain rates and pressures are reached. This fact discourages the application of this model to the case of study in this thesis, since strain rates above $10^{5} \mathrm{~s}^{-1}$ are only reached in very localized points and only during the duration of the proton beam burst.

The S-L model, on the other hand, is an improvement of the S-G model, extending its validity to strain rates as low as $10^{-4} \mathrm{~s}^{-1}$, in particular to be applied for bcc metals such as tantalum [187]. Unfortunately, the S-L models is not implemented in ANSYS AUTODYN $\AA$ complicating its use to the case of study in this thesis.

\section{Preston-Tonks-Wallace strength model}

An example of a more modern and complex physical based model applicable to a wide range of strain rates and temperatures is the Preston-Tonks-Wallace (PTW) model [188]. The PTW model estimates metallic plastic flow and is suitable for numerical simulations of explosive loading and high velocity impacts. In this model, the dynamic response of a material depends only on its current micro-structural state, which is characterized by its grain size distribution, the distribution of grain orientations, dislocation density, dislocation network structure, volume fraction of twins, etc. This is done through the definition of true internal state variables, following the approach of the previously existing mechanical threshold stress (MTS) model [189], in which it was developed a constitutive model employing the mechanical threshold stress (flow stress at $0 \mathrm{~K}$ ) as a structure parameter. The PTW model, on the other hand, extends the MTS model using the equivalent plastic strain as an approximate internal state variable, making it applicable (in addition to temperature dependence) to 15 orders of magnitudes of strain rate, from $10^{-3} \mathrm{~s}^{-1}$ to $10^{12} \mathrm{~s}^{-1}$. Ref. [188] includes model predictions and comparison with experimental data for a wide range of materials such as $\mathrm{Cu}, \mathrm{U}, \mathrm{Ta}, \mathrm{Mo}, \mathrm{V}, \mathrm{Be}$, 304 SS, together with the model parameters for these materials. However, the main drawback of this model is its complexity and that is not implemented yet in most of the commercial hydrocodes, such as in ANSYS AUTODYN $®$. 


\subsubsection{Failure Models}

After the introduction of EOS and strength models, the third element necessary to simulate real material behavior is a failure model. Materials cannot withstand local tensile stresses greater than its limits. For this reason, it is necessary to implement this phenomenon in the code in order to simulate a material response close to reality. This is done by the definition of a certain threshold which, when it is exceeded in an element, tells the code to consider that the material can no longer sustain any shear stress or tensile pressures in the corresponding element, just in the same way as it would happen in reality when a crack appears and there is no physical bonding between two parts in the cracked material.

There is a wide number of failure models formulations depending on the type of material, loading conditions, and assumptions made to define the mentioned threshold. In reality, dynamic fracture occurs mainly in two modes: brittle and ductile. Brittle fracture takes place by the propagation of a crack with a sharp front, whereas ductile fracture exhibits substantial plastic deformation, crack propagation require more energy and the failure occurs after a cumulative damage [147]. This classification divides the failure models used by the hydrocodes in two categories:

i Material instantaneously fails when one or a certain number of variables (such as strain to fracture, tensile hydrostatic stress, maximum principal stress) overcome a limit in a particular location.

ii Failure models are based on the cumulative damage of the material when a certain variable is repeatedly exceeded [159].

In the next sections some of these models are introduced.

\section{Strain to Failure, Maximum Shear Stress and Minimum Hydrostatic Pressure failure models}

In this section, three models belonging to the first group of failure models introduced in the previous paragraph are presented. Theses models are therefore associated to brittle failure since they assume that the element fails when a certain variable overcomes a given limit. In this context, the Strain to Failure Model assumes that the fracture occurs in an element when the equivalent plastic strain reaches a critical value $\epsilon_{f}$ as seen in eq. 2.46 [190].

$$
\epsilon_{f}=\sqrt{\frac{2}{3}} \sqrt{\epsilon_{1}^{2}+\epsilon_{2}^{2}+\epsilon_{3}^{2}}
$$


Even though this criterion is very simple and lacks generality, it has found its way into almost all nonlinear commercial codes since equivalent strain and data can be easily found in the literature. Similarly, for the Maximum Shear (MS) stress model the fracture is governed by eq. 2.47. This criterion considers that fracture may occur on a plane where the shear stress is maximum. This plane stands in the bisector angle created by the maximum and minimum principal stresses and the value of the shear stress there is equal to their semi-difference. This formulation is similar to the Tresca yield condition but in general $\tau_{f}$ defined here is larger than the yield stress in shear of Tresca [190].

$$
\tau_{f}=\max \left\{\frac{\sigma_{1}-\sigma_{2}}{2}, \frac{\sigma_{2}-\sigma_{3}}{2}, \frac{\sigma_{3}-\sigma_{1}}{2}\right\}
$$

Finally, other simple formulation especially relevant and used for shock loaded applications is the Minimum Hydrostatic Pressure failure model $\left(P_{\text {min }}\right)$. This model assumes that the material fractures when a given tensile pressure is exceeded. This tensile pressure is commonly obtained from impact tests on specimens in which spall is induced on a free surface of the tested material. Spalling is a fracture process characterized for a sudden fragmentation and ejection of particles taking place when a high compressive wave is reflected into a tensile one in a free surface. In this context, the minimum hydrostatic pressure model uses this tensile pressure as a threshold for failure. Spall pressure of several materials obtained by impact tests can be found in ref. [176]. Some numerical studies of material damage induced by beam impacts using this failure criterion can be found in the literature ([164], [166]). As with the previous two models, the $P_{\text {min }}$ failure model is implemented in most of the nonlinear commercial codes due to its simplicity and data availability in the literature.

\section{Grady Spall failure model}

Similarly to the $P_{\min }$ failure model recently described, the Grady Spall model can be used to simulate dynamic spall of metals under shock loading [191, 192]. This model has the advantage of being more physically based since it is derived from equilibrium balances between the surface energy and kinetic energy during the fragmentation process. In this way, the parameters associated to this model can be obtained from fundamental properties of the material. Eq. 2.48 shows the formulation of this model in the simple form in which it is implemented in ANSYS AUTODYN@ [159], although several more advanced formulations exist.

$$
S=\sqrt{2 \rho c^{2}} Y \epsilon_{c}
$$


where $S$ is the critical spall strength, $\rho$ is the density, $c$ is the bulk sound speed, $Y$ is the yield strength and $\epsilon_{c}$ is a critical strain value.

\section{Johnson-Cook Failure Model}

The J-C failure model [193] is a cumulative-damage fracture model (belonging to the second group in the classification of section 2.6.4) applicable to ductile materials. This model considers the influence in the failure of strain, strain rate, temperature and pressure. It takes into account the path dependence by accumulating damage during the deformation process. The material damage is evaluated by means of the damage parameter $D$, defined in eq. 2.49:

$$
D=\sum \frac{\Delta \epsilon}{\epsilon_{f}}
$$

where $\Delta \epsilon$ is the equivalent plastic strain increment and $\epsilon_{f}$ is the equivalent strain to fracture, which is defined in a similar way as in the Johnson-Cook strength model, consisting of three independent terms that define the dynamic fracture strain as a function of pressure, strain rate and temperature as shown in eq. 2.50.

$$
\epsilon_{f}=\left(D_{1}+D_{2} e^{D_{3} \sigma^{*}}\right)\left(1+D_{4} \ln \frac{\dot{\epsilon_{p}}}{\dot{\epsilon_{0}}}\right)\left(1-D_{5}\left(\frac{T-T_{r}}{T_{m}-T_{r}}\right)\right)
$$

Where $\sigma^{*}$ is defined as $\frac{-P}{\sigma_{V M}}$ and $D_{1 \ldots 5}$ are constant parameters obtained by fitting experimental and numerical data of tensile and torsion tests. The damage parameter $D$ in certain elements is continuously updated during the calculation until it reaches the value of 1 , which corresponds to the material being fractured. 


\subsection{Computational Method for the Resolution of Dynamic Response of the AD-Target}

In the introduction of Chapter 1 it was identified that the thermo-mechanical response and stress waves produced in the target core as consequence of the sudden deposition of energy by the primary proton beam is one of the main issues which limit the target life and influence the $\bar{p}$ production yield. As a first attempt to resolve these conditions, section 2.4 of the present chapter showed the application of conventional thermo-mechanical simulations using implicit-FE to this problem. This approach, however, has been shown to be limited in producing accurate results in a computationally efficient way due to the strong assumptions involved as demonstrated in section 2.5. On the other hand, hydrocodes, described in detail in section 2.6, have been proven to be a powerful computational tool to overcome these assumptions and produce valuable insights of the core dynamic response and its structural state.

In this section, the application of hydrocodes for this purpose is presented, including the computational approach and the characteristics of the material models used.

\subsubsection{Computational Approach}

Figure 2.9 shows a schematics of the methodology employed in this study. First, an energy deposition map in the target material as a consequence of the proton beam-target atoms interaction is calculated by means of FLUKA[194] Monte Carlo simulations. This energy deposition map is then applied as an internal energy

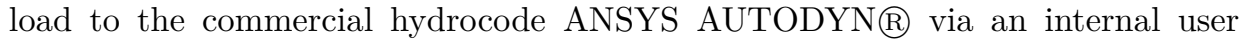
subroutine and the user function EXSIE3. The energy is applied consistently with the PS beam parameters, which proton beam is composed by 4 proton bunches spaced by $105 \mathrm{~ns}$, bunch length of $30 \mathrm{~ns}$, and total intensity of $1.5 \cdot 10^{13}$ protons per pulse [10]. The total energy deposited in the target core is approximately 1.34 $\mathrm{kJ}$, which results in $11.17 \mathrm{GW}$ considering that it is deposited in only $120 \mathrm{~ns}$ (4 bunches x 30 ns duration). Moreover, the power is applied in the small volume of the target core $\left(\sim 0.4 \mathrm{~cm}^{3}\right.$, producing a mean power density of $2.87 \cdot 10^{4} \mathrm{TW} / \mathrm{m}^{3}$ (even if the deposition is not uniform).

It is important to note that the interaction between FLUKA and AUTODYN@ is only a one-way coupling, which means that the reduction in the deposited energy during the pulse impact as a consequence of the change of target material density is not taken into account. This is considered a valid assumption as the maximum decrease of density during the $430 \mathrm{~ns}$ pulse burst due to the increase of temperature is in the order of $2 \%$ of the initial density, since the material is not melting. 


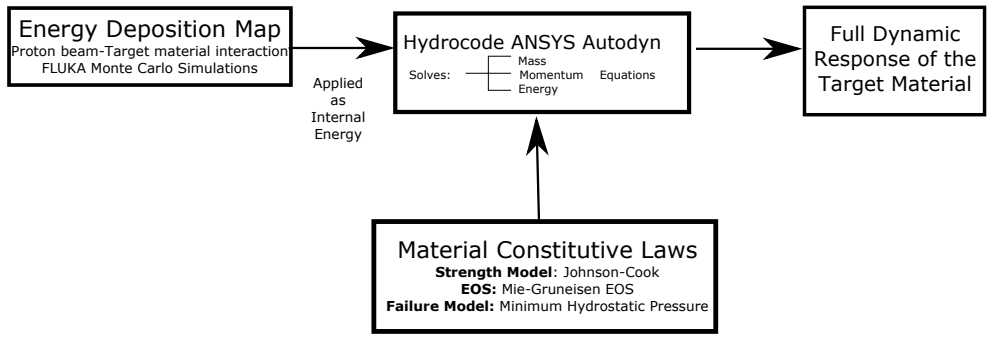

Figure 2.9: Schematics of the methodology employed in this study, including the two computational tools FLUKA and ANSYS AUTODYNR

\subsubsection{Geometry and Computational Domain}

Simulations are performed using the reference geometry shown in Figure 2.10 (a), which corresponds to the target core and containing graphite matrix. In addition to this configuration, two others have been investigated, including a copper and tantalum cladding up to $1 \mathrm{~mm}$ thickness around the target core, (Figure 2.10 (b) and (c)). The aim of these simulations was to investigate the influence of the impedance mismatch between target core and surrounding materials on the pressure wave response inside the target, which could be an efficient way to reduce the magnitude of tensile pressure reached in the target. This is explained more in detail in section 2.8.3.

The post processing tool of AUTODYN® allows to add gauges (probes) in specific points of the geometry in order to check the time history of a selected variable. The data presented in the plots of the simulation results in Chapter 3 corresponds to the position of the gauges shown in the figure 2.10. The computational domain consisted in an unstructured mesh with a total of 350000 hexahedra elements, (215000 in the target core and 135000 in the graphite matrix). Only hexahedra elements were used since the subroutine for the interpolation of energy depositions from FLUKA simulations only works with such element type.

ANSYS AUTODYN $®$ provides a good variety of solvers for the numerical resolution of mass, momentum and energy equations, enabling it to obtain the full dynamic response of the material. For the present study, a Lagrangian solver is used as the deformation of the material is produced by its thermal expansion and no important mesh distortions occur.

It must be mentioned at this point that most of the simulations presented in this work were carried out assuming tungsten instead of iridium as target core material. This assumption had to be made due to the lack of strength and failure models of iridium in the literature. In parallel to of these calculations, an especial effort was put in order to obtain strength models of iridium. For this reason, a dynamic characterization campaign was launched at DYNLab in Politecnico di 


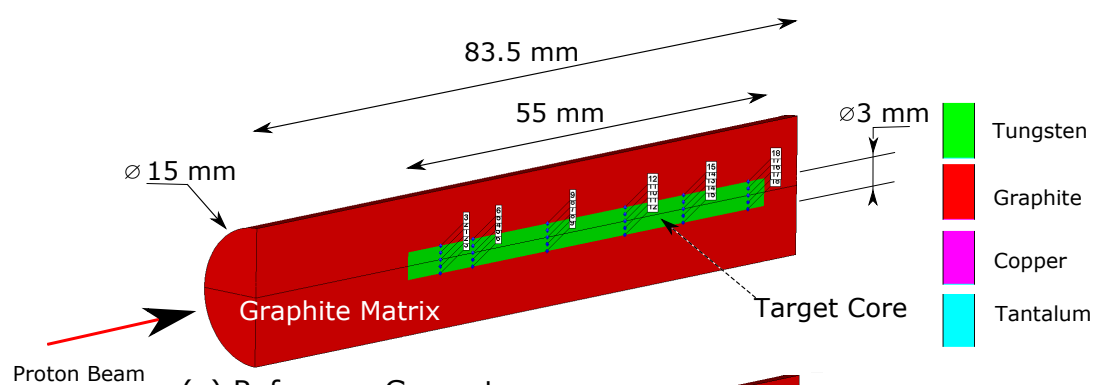

Proton Beam (a) Reference Geometry

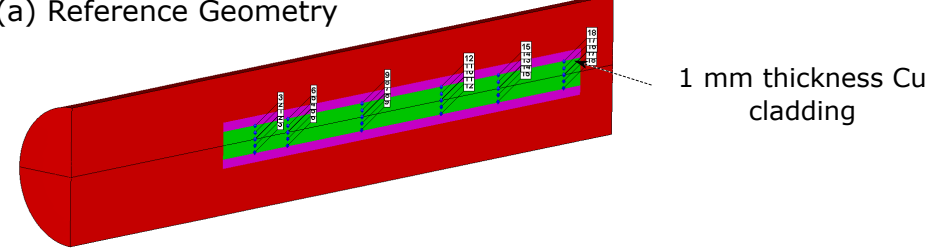

(b) Copper Cladding

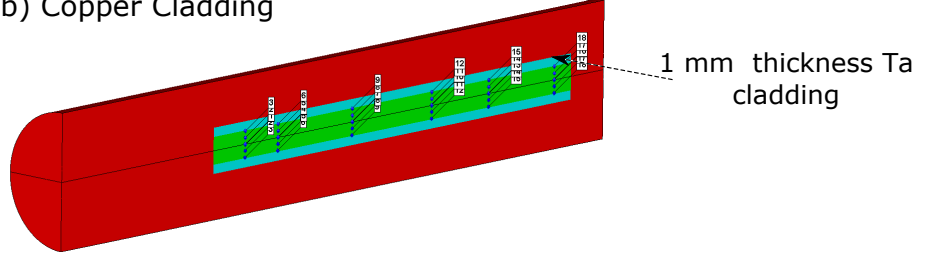

(c) Tantalum Cladding

Figure 2.10: Half-view of the different configurations studied in the present work: (a) $\mathrm{W}$ target surrounded by graphite matrix (b) $\mathrm{W}$ target with $\mathrm{Cu}$ cladding surrounded by graphite matrix (c) W target with Ta cladding surrounded by graphite matrix.

Torino [195] with the aim of extracting the experimental parameters necessary of a strength model for iridium. This campaign is explained more in detail in section 2.7.3. Nevertheless, tungsten is a strong candidate material and the lessons learned from this numerical study (presented in Chapter 3) depend mainly on the pure dynamic response, making possible to extrapolate theses conclusions to other candidate materials (such as iridium). In addition, a brief comparison between the results obtained for tungsten and iridium (once the data obtained from this characterization campaign was available) is provided in section 3.7, demonstrating the validity of this tungsten-iridium extrapolation.

From the point of view of physical constraints, the model is considering that the external graphite envelope can freely expand in every dimension. This assumption does not affect at all the dynamic response of the center core in the time window of interest, as the phenomena of study are so fast that there is not enough time for the speed of sound to travel to and come back from the periphery of the computational domain (the external graphite envelope). 


\subsubsection{Employed EOS, Strength and Failure Models}

As explained in detail in sections 2.6.1-2.6.4, there are three main ingredients necessary to perform hydrocode calculations; Equation of State (EOS), Strength Model and Failure Model. In this section a discussion of the selected models for the performed calculations is included. The model selection is also summarized in table 2.4 .

From the point of view of EOS, a Mie-Grüneisen is employed for all the materials. As introduced in section 2.27, this is one of the most widely used EOS and one of which more parameters are available in the literature, working especially well in the regime of not very high pressures, where the relation between shock speed and particle speed can still be considered linear (less than a few hundred gigapascals in pressure [147]). The AUTODYN@ implementation of Mie-Grüneisen takes as well into account differences between compressive and tensile states. This formulation does not take into account change of phase, which is perfectly valid for the case under study, where melting of material does not occur due to the high melting point of the candidate materials, and the pressures reached are in the order of GPa's, which in terms of shock physics, are relatively low. The parameters for these EOS models were obtained from the material database already available in AUTODYN® except the one of graphite, which was not available and therefore was obtained from ref. [176].

On the other hand, the strength model selection is one of the most complex aspects in the construction of the hydrocode model. From an ideal point of view, it would be desirable to use a physical-based strength model or an experimental strength model that has been extracted in the regime of operation. However, this is very difficult to achieve in practice. Physical based strength models such as PTW or MTS models are neither implemented in AUTODYN® nor their necessary parameters for rare materials, such as iridium, are available in the literature. In addition, even if using an experimental based strength model such as J-C, S-G or Z-A (which are implemented in AUTODYN@), experimental parameters for the temperature and strain rate regime reached in the target core (up to $2000{ }^{\circ} \mathrm{C}$ and above $10^{4} s^{-1}$ ) are not available. For this reason, assumptions have to be made and the selected strength models have to be partially extrapolated. Validation of this extrapolation is one of the main motivations of the experiment presented in Chapters 4,5 and 6 of this thesis.

In this context, a J-C strength model was used for tungsten, which was obtained from ref. [70]. The parameters for this model were extracted by a servo hydraulic load machine and compression Kolsky bar testing in a range of $10^{-3}-10^{4} s^{-1}$ strain rates and up to $800{ }^{\circ} \mathrm{C}$. In the case of pure iridium, not a single dynamic testing at high strain rate has been found in the literature. The closest studies found are dynamic tests of iridium alloy DOP-26 in refs. [66, 64, 65] (an iridium alloy developed at Oak Ridge National Laboratory containing $0.3 \%$ of $\mathrm{W}$ and 
Table 2.4: Material Models employed by the simulations

\begin{tabular}{|c|c|c|c|}
\hline Material & EOS & Strength Model & Failure Model \\
\hline Tungsten & Mie-Grüneisen & $\mathrm{J}-\mathrm{C}[70]$ & $\begin{array}{c}\text { Minimum Hydrostatic } \\
\text { Pressure } \\
P_{\text {min }}=-2.6 \mathrm{GPa}[196] \\
{ }^{*} \text { Benchmarked in Chapter } 6 \\
P_{\text {min }}=-0.85 \mathrm{GPa}\end{array}$ \\
\hline Graphite & $\begin{array}{l}\text { Mie-Grüneisen } \\
\text { from ref. [176] }\end{array}$ & Viscoelastic [197] & $\begin{array}{c}\text { Principal Tensile } \\
\text { Failure Strain }[197] \\
\sigma_{\text {fail }}=6 \cdot 10^{-3}\end{array}$ \\
\hline Copper & Mie-Grüneisen & $\mathrm{J}-\mathrm{C}$ & - \\
\hline Tantalum & Mie-Grüneisen & von Mises or J-C [78] & - \\
\hline Iridium & Mie-Grüneisen & $\mathrm{J}-\mathrm{C}[67]$ & $\begin{array}{c}{ }^{*} \text { Benchmarked in Chapter } 6 \\
P_{\text {min }}=-0.9 \mathrm{GPa}\end{array}$ \\
\hline
\end{tabular}

approximately $40 \mathrm{ppm}$ of Th to improve grain boundary cohesion at high temperatures) which include temperature dependent tests up to $1000{ }^{\circ} \mathrm{C}$ and $10^{4} \mathrm{~s}^{-1}$ strain rates. Unfortunately, these publications do not include either data fitting or extraction of strength models and they are done in DOP-26 alloy, whose response can be significantly different than the one of pure iridium. It is for this reason that a campaign was launched in collaboration with Politecnico di Torino from which parameters of a J-C model for pure iridium were obtained up to $1200{ }^{\circ} \mathrm{C}$ and $10^{4} \mathrm{~s}^{-1}$. These parameters were used in the simulations. More details of these dynamic tests can be found in section 2.7.3.

For the case of tantalum, when used in the scenario where Ta-cladding was added to the target core, a bi-linear strength model was employed, defined in AUTODYN with the name of von Mises. This model was considered suitable, even if it is not temperature or strain rate dependent, since the interest of the simulations was on the stresses reached in the target core rather than in the cladding itself. It was ob- 
served by simulations that the yield of the cladding material does not significantly influence the core response (only a difference of $7 \%$ in the maximum tensile pressure reached was observed between simulations considering Ta yielding versus a perfect elastic material). In addition, temperatures reached in the Ta-cladding are moderate $\left(250-400{ }^{\circ} \mathrm{C}\right)$ in comparison with the ones reached in the core $\left(2100{ }^{\circ} \mathrm{C}\right)$. Nevertheless, for simulations considering the response of experimental Ta targets studied in Chapters 4-6, a J-C model extracted by Split Hopkinson bar tests up to $3800 s^{-1}$ and $1000{ }^{\circ} \mathrm{C}[78]$ was used.

For graphite, a visco-elastic strength model from ref. [197] was used in order to take into account the strain rate dependence of this material and its high energy dissipating component, in terms of wave damping and dispersion effects due to its porous nature.

Regarding the failure model, there is also a lack of parameters and models in the literature for the materials of interest at the conditions reached, similarly to what is happening with the strength models. For this reason, a simple model as the Minimum Hydrostatic Pressure failure model is used for the case of tungsten as target core material. The value of this limiting tensile pressure was assumed to be -2.6 $\mathrm{GPa}$, obtained experimentally in laser induced free surface spall in polycrystalline tungsten [196]. No such data exists for iridium, hence, no failure model has been implemented for this case in the simulations presented in Chapter 3. Nevertheless, a failure model benchmarking for iridium and tungsten was performed in Chapter 6 from the experimental results presented in Chapter 5. This benchmarking gave a minimum pressure of failure of $-0.85 \mathrm{GPa}$ and $-0.9 \mathrm{GPa}$ for tungsten and iridium respectively. For the potential fracture of the graphite matrix, a minimum tensile principal strain failure model obtained from spall experiments at room temperature using a hopkinson pressure bar setup in ref. [197] was used. According to this reference, the failure tensile strain of spall for graphite was found to be $6 \cdot 10^{-3}$. Regarding the failure model, there is also a lack of parameters and models in the literature for the materials of interest at the conditions reached, similarly to what is happening with the strength models. For this reason, a simple model as the Minimum Hydrostatic Pressure failure model is used for the case of tungsten as target core material. The value of this limiting tensile pressure was assumed to be -2.6 $\mathrm{GPa}$, obtained experimentally in laser induced free surface spall in polycrystalline tungsten [196]. No such data exists for iridium, hence, no failure model has been implemented for this case in the simulations presented in Chapter 3. Nevertheless, a failure model benchmarking for iridium and tungsten was performed in Chapter 6 from the experimental results presented in Chapter 5. This benchmarking gave a minimum pressure of failure of $-0.85 \mathrm{GPa}$ and $-0.9 \mathrm{GPa}$ for tungsten and iridium respectively. For the potential fracture of the graphite matrix, a minimum tensile principal strain failure model obtained from spall experiments at room temperature using a hopkinson pressure bar setup in ref. [197] was used. According to this reference, the failure tensile strain of spall for graphite was found to be $6 \cdot 10^{-3}$. 


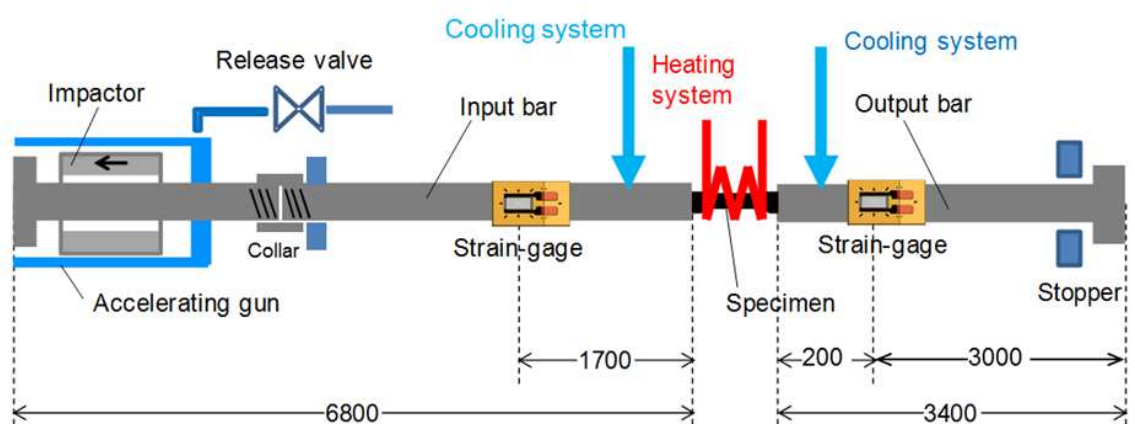

Figure 2.11: Schematic of a Split-Hopkinson Pressure Bar used in DYNLab for the dynamic characterization of Iridium at high strain rates [67]. Distances in mm. Courtesy of L. Peroni and M. Scapin.

\section{Extraction of J-C Parameters of Iridium by Dynamic Testing}

As introduced previously in this chapter, the limited availability of strength model parameters in the literature (in particular for iridium) is one of the main constraining factors for the accurate numerical assessment of the dynamic response of the AD-Target. For this purpose, a collaboration between CERN and DYNLab at Politecnico di Torino [195] was established. The aim of this collaboration was to perform dynamic tests in iridium at the widest possible range of temperature and strain rates and, in this way, extract strength model parameters to be applied in the hydrocodes simulations.

A series of tests from room temperature up to $1200^{\circ} \mathrm{C}$ and at different strain-rates were performed at DYNLab in order to obtain information about temperature and strain-rate sensitivity of iridium. The strain-rate sensitivity was investigated starting from $10^{-3} s^{-1}$ up to $10^{4} s^{-1}$ [67]. The medium-low strain rate tests were performed using an electro-mechanical testing machine. The high strain-rate conditions were reached by means of Hopkinson Bar setups in direct configuration. Figure 2.11 shows a scheme of the configuration of the Split Hopkinson Pressure Bar (SHPB) used to test the specimens in tensile (courtesy of L. Peroni and M. Scapin). In this kind of tests, the specimen is screwed between two long bars, called incident bar (or input) and transmitted bar (or output), respectively. At the extremity of the incident bar a gas-gun driven hollow flyer is impacted, generating an elastic longitudinal tensile stress wave traveling along the incident bar. This tensile wave is transmitted to the specimen and subsequently to the transmitted bar. Two strain gauges are placed, one on the impacted bar and one on the transmitted one. It can be shown that it is possible to obtain the stress-strain curve of the dynamically loaded material just from the strains recorded in the incident and transmitted bars. The force applied to the specimen is obtained from the young modulus of the impacted bar, the recorded strain, and the section areas 


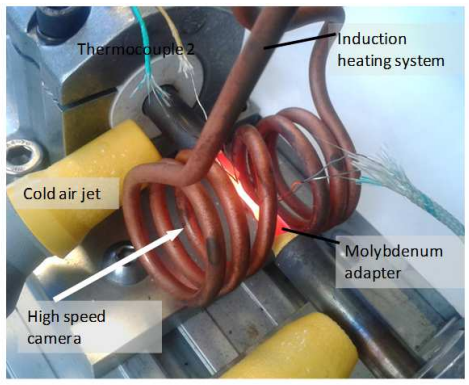

(a)

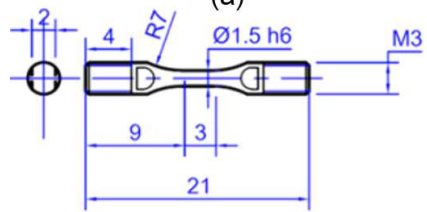

(c)

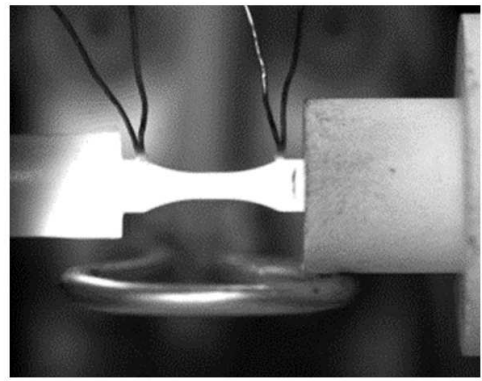

(b)

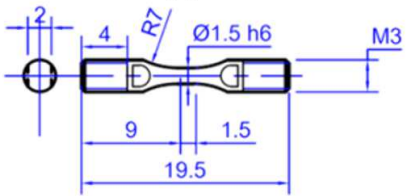

(d)

Figure 2.12: (a)-(b) Picture of the heated miniaturized Ir specimen, placed between the incident and transmitted bars [67]. (c)(d) Schematics of the specimens for tests up to $10^{3} s^{-1}$ and $10^{4} s^{-1}$ respectively. Courtesy of L. Peroni and M. Scapin.

of the bar and specimen. The strain at location of the specimen can be inferred from the difference between the recorded strains at the incident and transmitted bars. The specimens were heated using an induction coil system, controlled with a feedback on the temperature measurement obtained by thermocouples directly welded on the specimen surface, as shown in figures 2.12-(a)(b).

In general, high strain-rates using a Hopkinson bar setup can be achieved in two ways. One possibility is to increase the speed of the striker bar, but this also increases the stress level in the bar, which is limited by the yield strength of its material. Another possibility is the reduction of the specimen dimensions [198]. For this reason, two different specimen geometries were used depending on test conditions. For quasi-static and dynamic tests up to $10^{3} \mathrm{~s}^{-1}$, specimens with gauge lengths of $3 \mathrm{~mm}$ and $1.5 \mathrm{~mm}$ diameter were used (figure $2.12(\mathrm{c})$ ). For dynamic tests at $10^{4} \mathrm{~s}^{-1}$, miniaturized samples with gauge length of $1.5 \mathrm{~mm}$ and $1.5 \mathrm{~mm}$ diameter were employed. Figure 2.13 shows a picture of the miniaturized specimens before testing. Due to the high price of the material, a significant optimization effort was put in order to manufacture the specimens from the minimum raw material. Manufacturing the specimens was not a straight forward process either, due to the extremely high hardness of iridium and lack of experience and applications, which difficult substantially its machineability process. Machining of the specimens was finally carried out by starting from rods of $4 \mathrm{~mm}$ diameter, and grinding with silicon-carbide wheels and diamond wheels. The threads of the specimens were done by electro-discharge machining (EDM). 


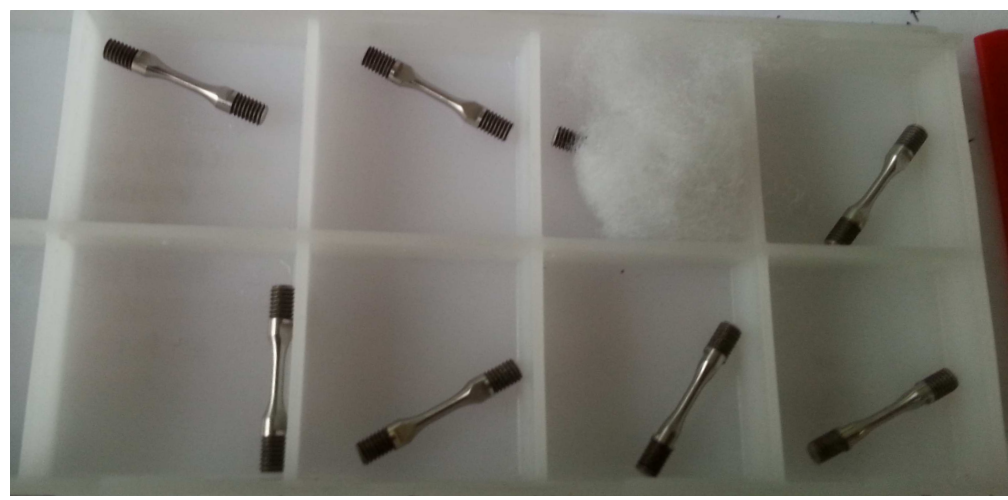

Figure 2.13: Picture of a set of 8 miniaturized specimens $-19.5 \mathrm{~mm}$ length- of pure iridium as received from manufacturing, before dynamic testing.

\subsection{Configurations and Objectives of the Performed Simulations}

\subsubsection{From Simple Assumptions to Complex Models}

For a deep understanding of the stress wave phenomena and interpretation of the results, the simulations were performed with a step by step approach, increasing gradually the level of complexity of the models, i.e. from a single material (only the target core) to multiple materials (graphite matrix and cladding), as well as from assumptions of perfectly elastic material response, up to implementation of plastic models and finally, failure models. This allows to separately identify the cause of different phenomena, such as the nature of the different waves involved, which origin could not have been noticed given the complexity of simulations where everything is taken into account. Sections 3.2, 3.3 and 3.6 of the next chapter show this progressive application of models; from elastic assumption, plasticity, to failure.

\subsubsection{Parametric Analysis of Influence of Proton Pulse Length on the Pressure Response}

From the analysis of the target core response when impacted by the proton beam, it will be identified the existence of an end-of-bunch tensile wave taking place due to the inertia effects. For this reason, a parametric analysis has been carried out in order to investigate whether these tensile waves can have an important influence on the overall response. In this analysis, proton impacts with different pulse length 
are considered. The total energy applied for each of the scenarios is the same, with the only difference of the duration of the pulse. Pulse lengths from $0.1 \mu$ s to $1.2 \mu \mathrm{s}$ in steps of $0.2 \mu \mathrm{s}$ have been considered and compared with the reference pulse $(0.430 \mu \mathrm{s}$ length $)$. A J-C strength model including plastic deformation of the material is used for this analysis as indicated in table 2.4. Section 3.4 shows this analysis.

\subsubsection{Parametric Analysis of Influence of Core Cladding on the Pressure Response}

Furthermore, in addition to the reference configuration currently present in the AD-Target, two extra configurations were studied, including a metallic cladding of $2 \mathrm{~mm}$ thickness around the core of the target (figure 2.10(b-c)). This cladding is added as a strategy to decrease the magnitude of the tensile pressure in the center of the target core. It is well known in the shock and explosive physics literature that the use of multi-layer armatures can effectively reduce the shockinduced tensile stresses in explosively expanding materials [199]. The key point resides in the acoustic impedance mismatch between the different materials in a multi-layered medium, which governs the fraction of the pressure wave energy that is transited/reflected between them [200]. The greater the impedance mismatch, the greater the portion of the shock energy reflected at the boundary. While, with a reduced mismatch a greater portion is transmitted to the adjacent medium. The ideal situation from a shock wave view point would be that no impedance mismatching exists between the target core and surrounding material, therefore all the energy of the radial pressure wave is transmitted to the adjacent media instead of being reflected to the core as a tensile and destructive wave. This possibility is studied numerically in the section 3.5 of the next chapter by assuming three different scenarios:

- No cladding between the $3 \mathrm{~mm}$ diameter high density target core and the surrounding graphite matrix (Figure 2.10-a). This is the case of the current design of the target, where the impedance mismatch is significant due to the large difference between tungsten and graphite density and sound velocity ( $\sim 30$ times ratio in acoustic impedance between target core and graphite)

- Copper cladding of $1 \mathrm{~mm}$ thickness (Figure 2.10-b). ( $\sim 2$ times ratio in acoustic impedance between target core and copper)

- Tantalum cladding of $1 \mathrm{~mm}$ thickness (Figure $2.10-\mathrm{c}) .(\sim 1.4$ times ratio in acoustic impedance between target core and tantalum)

For these calculations, strength models which take into account the response beyond plasticity are considered as well for the cladding materials as shown in table 2.4. No failure model is considered, in order to be able to see the propagation of 
the wave. The contact boundary between the target core and surrounding materials is assumed to be perfect by the code, i.e. the mesh is considering a continuous body with shared nodes at the interface, so no contact algorithm is necessary. This is done to avoid sensitivity of the mesh and the non-linearity of the contact algorithm parameters for the comparison between results, reducing the problem to a pure dynamic phenomenon. In reality, such kind of contact could be achieved by diffusion bonding executed via HIP (Hot Isostatic Pressing) process.

\subsubsection{Comparisons of Iridium and Tungsten as Core Material}

As stated before, most of the calculations presented in Chapter 3 are done considering tungsten as target material due to initial limitation of availability of strength and failure models of iridium. Nevertheless, after the dynamic testing and extraction of a J-C strength model of iridium explained in section 2.7.3, a cross-check analysis between response of iridium and tungsten as core material has been carried out. The goal of this cross-check is to demonstrate the validity of the main results obtained in the previous configurations where tungsten was assumed as core material. This analysis is presented in section 3.7.

\subsubsection{Analysis of Dynamic Response of the Graphite Matrix}

Finally, a detailed analysis of the dynamic conditions reached in the graphite was carried out and is presented in section 3.8. The goal of this analysis was to understand which is the level of propagation of the wave generated in the core to the graphite matrix. A Minimum Tensile Principal Strain failure model obtained from spall experiments was introduced in the graphite in an attempt to estimate the extent of damage that this propagated wave could produce in the matrix. 


\section{Chapter 3}

\section{Results of the Dynamic Response of the Target Core under Proton Beam Impact}

In Chapter 1 an introduction to the characteristics and operation concerns of the AD-Target was presented, identifying the dynamic response of the core as one of the main concerns which could limit the target life and influence the $\bar{p}$ production yield. For this reason, it was considered of prime importance to study in detail this response using the best available computational tools in order to reduce the uncertainties concerning the target core structural state.

In Chapter 2, preliminary calculations using widespread implicit FE numerical methods were performed as a first approach. It was demonstrated, however, that conventional implicit FE is limited in producing accurate results for the case of application. Hydrocodes, on the other hand, seem to be the appropriate computational tool to numerically resolve the extreme conditions reached, taking into account material response beyond plasticity, temperature and strain rate dependency, and possible fragmentation. A detailed description of how hydrocodes work was included, together with a general literature review of existing material models and their peculiarities. In addition, the employed methodology for the application of hydrocodes to the AD-Target was defined, including the material model selection and the different scenarios of interest. In the present chapter, the results of the simulations performed in these scenarios, described in section 2.8, are included. 


\subsection{Adiabatic Increase of Temperature in the Target}

As already anticipated in section 2.4, an increase of temperature of up to $2000{ }^{\circ} \mathrm{C}$ takes place at the center of the target core during each proton beam impact as a consequence of the proton-target material interaction. Figure 3.1 shows the temperature profile in a $3 / 4$ cut view of the target core at the end of a single proton pulse impact, i.e. at $430 \mathrm{~ns}$. This simulation assumes tungsten as core material instead of iridium. For this reason, temperatures reached here are slightly lower than the ones presented in section 2.6, since tungsten is less dense and has a lower atomic number ( $\mathrm{Z}$ ) than iridium, and therefore the energy deposited by the beam is lower. Apart from this, there is no significant difference between this result when using hydrocodes and the one presented in section 2.4 using ANSYS Mechanical@, since the thermal solver of ANSYS-implicit is perfectly capable to accurately calculate the adiabatic increase of temperature in the target. Even more accurately than

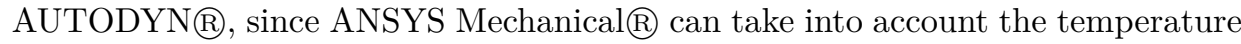
dependence of the specific heat, while in AUTODYN $\mathrm{R}$ this property is considered constant. Nevertheless, it should be mentioned that AUTODYN® works in terms of internal energies, therefore the temperature is just an output variable, only sometimes used in particular strength models. The expansions associated to the increase of internal energy is accurately predicted by the EOS, independently from the constant specific heat defined and the temperatures shown.

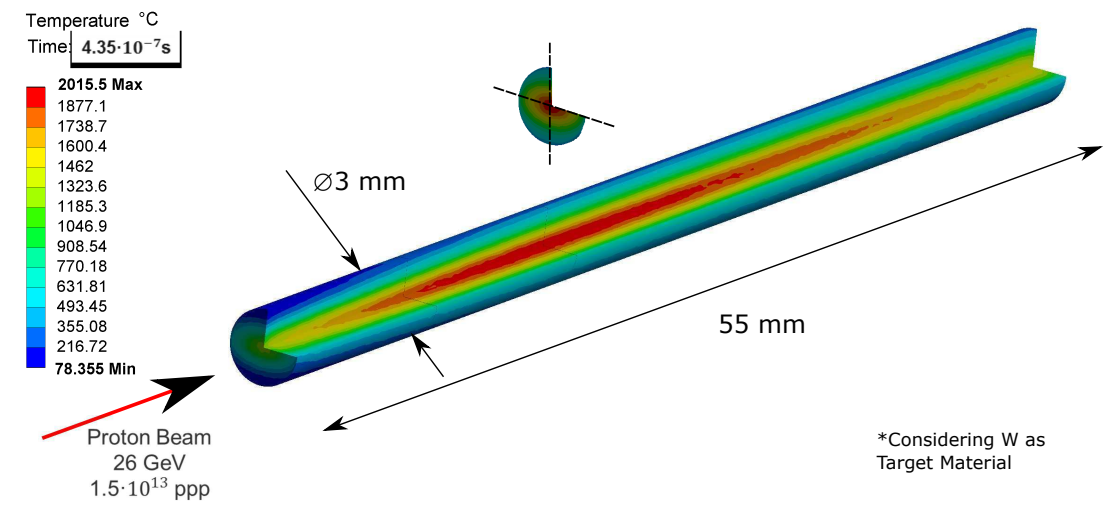

Figure 3.1: $3 / 4$ cut view of the temperature profile in the target core at the end of a single $26 \mathrm{GeV}$ proton beam impact considering tungsten as target material instead of iridium. The maximum temperature reached is therefore slightly lower, $2015{ }^{\circ} \mathrm{C}$ instead of the $2110{ }^{\circ} \mathrm{C}$ shown in figure 2.6 . 


\subsection{Elastic Response of the Target Core}

Figures 3.2 and 3.3 show respectively the short and long transient pressure response in the center of the target core and periphery (gauges 7 and 9 in figure 2.10), assuming a perfect elastic material. This assumption is by far unrealistic as tungsten's yield strength is no more than $800 \mathrm{MPa}$ above $500^{\circ} \mathrm{C}$, even in the most favorable manufactured condition[101], which are well exceeded in the target core. Therefore, the pressure values shown in these plots can only be taken into account in a qualitative way, since the energy diffused by plastic deformation processes is not considered. Simulations assuming elastic material, however, allow a better understanding of the pressure wave propagation, as no damping of the waves in the material occurs.

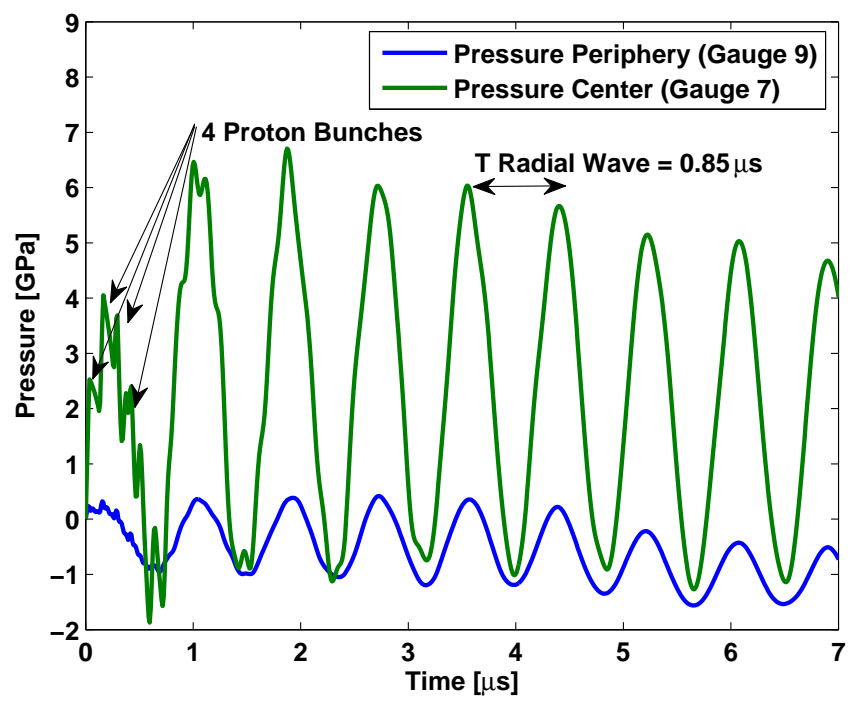

Figure 3.2: Pressure response at the center (gauge 7) and periphery (gauge 9) during the first $7 \mu$ s after the $430 \mathrm{~ns}$ (four bunches) proton pulse impact under purely elastic material assumption (displayed pressures only for qualitative information). The plot clearly shows the excitation of a radial wave (or radial mode) with a $0.85 \mu$ s period. Note that the oscillation takes place in phase in the center and periphery, meaning that the rod material simultaneously expands and contracts within its transverse section.

Figure 3.2 shows the pressure response in the target during the first $7 \mu \mathrm{s}$. A four stepped rise of pressure corresponding to the four impacts of four proton bunches spaced by 120 ns can be observed. Subsequently, a clear high frequency pressure wave response takes place. This wave has a period of $0.85 \mu \mathrm{s}$ and it matches very well the analytically predicted value for radial modes period shown in table 2.1 of Chapter 2. The pressure wave in the center and periphery (gauges 7 and 9 
in figure 2.10) are in phase, meaning that the whole section of the rod expands and shrinks simultaneously as introduced in the description of radial modes of section 2.2.2. This is due to the fact that the sudden rise of temperature in the material does not only happen at the center but also at the periphery of the rod, where the increase of temperature is also significant (above $300{ }^{\circ} \mathrm{C}$, as can be seen in figure 3.1). The consequence is that the pressure waves are not just traveling from a central, localized area, but there are infinitesimal pressure waves with infinitesimal departing points along the radius of the rod. The amplitudes of these pressure waves are attenuated as they travel to the peripheral surface, and amplified as they do it through the center due to the cylindrical geometry. The so called radial mode, introduced in section 2.2.2, is the result of the interactions of all these waves at each single point, leading to the pressure (and other variables) oscillations observed in figure 3.2 .

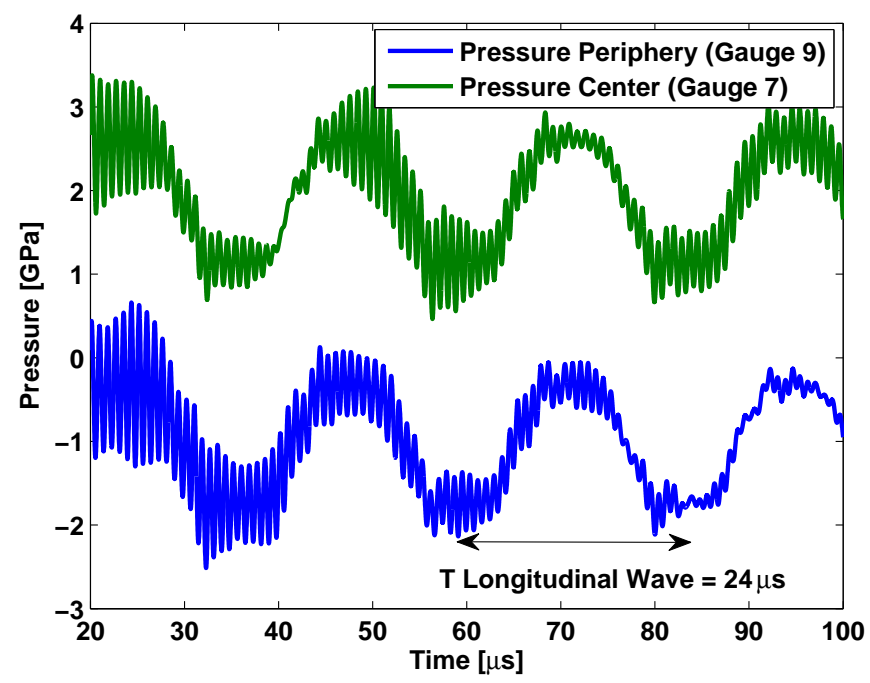

Figure 3.3: Pressure response at the center (gauge 7) and periphery (gauge 9) during the $20 \mu \mathrm{s}-100 \mu$ s period, under purely elastic material assumption (displayed pressures only for qualitative information). The plot clearly shows the presence of high frequency radial and low frequency $-24 \mu$ s period- longitudinal waves.

Figure 3.3 shows the response of the transient analysis in an expanded time window, from $20 \mu$ s to $100 \mu \mathrm{s}$, allowing to observe, in addition to radial high frequency wave, a longitudinal pressure wave. This wave has a period of $24 \mu \mathrm{s}$, which corresponds to the excitation of a longitudinal mode and was accurately predicted in the table 2.2 of Chapter 2. These mode results from the interaction of infinitesimal waves traveling along the $55 \mathrm{~mm}$ longitudinal distance. It is important remark to that, as anticipated in Chapter 2, these longitudinal pressure waves are in phase 
at all the longitudinal points of the rod. This can be easily noticed in figure 3.4, where the longitudinal displacement at different longitudinal points of the rod axis (gauges 1, 4, 7, 10, 13, 16 in figure 2.10) is shown. The coordinate reference system in the model is placed at central transversal section of the rod, higher displacements are symmetrically reached at gauges 1 and 16, which are close to each of the rod ends, while displacement is reduced when symmetrically approaching the central transversal section of the rod (gauges 7 and 10). The whole rod is therefore expanding longitudinally in a simultaneous way within all its volume, in a similar manner as it was happening radially. The reason of the excitation of this mode, again, is that the temperature rise caused by the deposited energy is also covering a great part of the longitudinal volume of the rod.

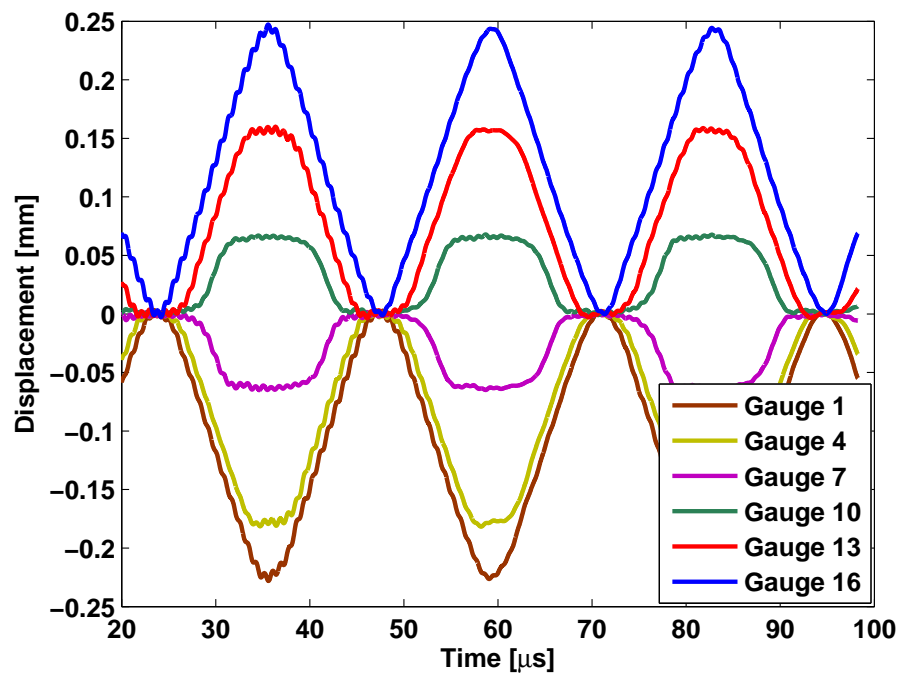

Figure 3.4: Axial displacement at different longitudinal positions of the target core after a proton pulse impact and under purely elastic material assumption. The plot clearly shows how the material of the target core expands and contracts longitudinally with a period of $24 \mu \mathrm{s}$, corresponding to the excitation of a longitudinal mode. 


\subsection{Plastic Response of the Target Core}

Figure 3.5 shows the pressure response during the first $7 \mu$ s with a simulation applying a J-C strength model, which, as described in section 2.6.3, takes into account plasticity, temperature and strain rate dependence. This simulation is therefore much more realistic than the one presented in the previous section, which assumed a purely elastic model. Figure 3.5 shows how the pressure wave experiences a fast damping due to the energy loss associated to the plastic deformation processes, which produces an attenuation of the wave in just $20 \mu \mathrm{s}$. This quick attenuation prevents the appreciation of the lower frequency longitudinal waves.

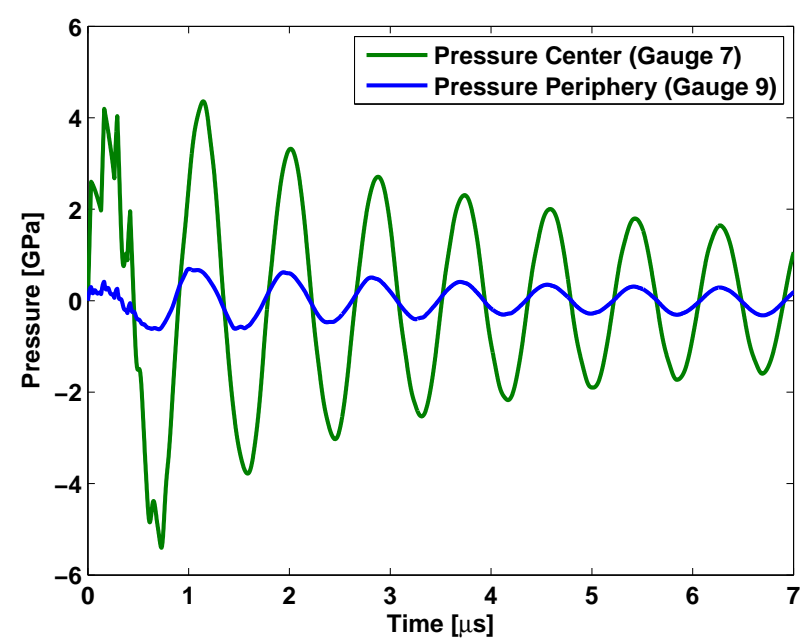

Figure 3.5: Pressure response at the center (gauge 7) and periphery (gauge 9) of the target core after a $430 \mathrm{~ns}$ proton pulse impact. This simulation considers J-C strength model, which takes into account the material response beyond plasticity. Note the change in the pressure distribution in comparison to the pure elastic material assumption (figure 3.2$)$.

In addition, another important difference concerning the wave response with respect to the elastic assumption is the compressive-to-tensile nature of the wave, reaching values of up to $4 \mathrm{GPa}$ (compression) and -5 GPa (tension) at the center of the rod during the first oscillations. This compressive-to-tensile response takes place also at the periphery, but with significantly lower amplitudes. This important difference in pressure distribution compared to the elastic assumption (figures 3.2 and 3.3) where the oscillations of pressure at the center of the rod were taking place always in compressive states, is due to the limitation of the deviatoric component in the stress tensor by the plastic limit of the material. The proper predictions of these high tensile states are fundamental, since they are sig- 
nificantly above the spall strength of the material and could cause fracture in the internal part of the target rod, producing a high loss of density which would lead to a significant decrease of antiproton yield. This fact also states the importance of using proper strength models, which take into account the material response beyond plasticity, since wrong assumptions will not only affect the magnitude of stress-strain response, but can change completely its distribution along the geometry.

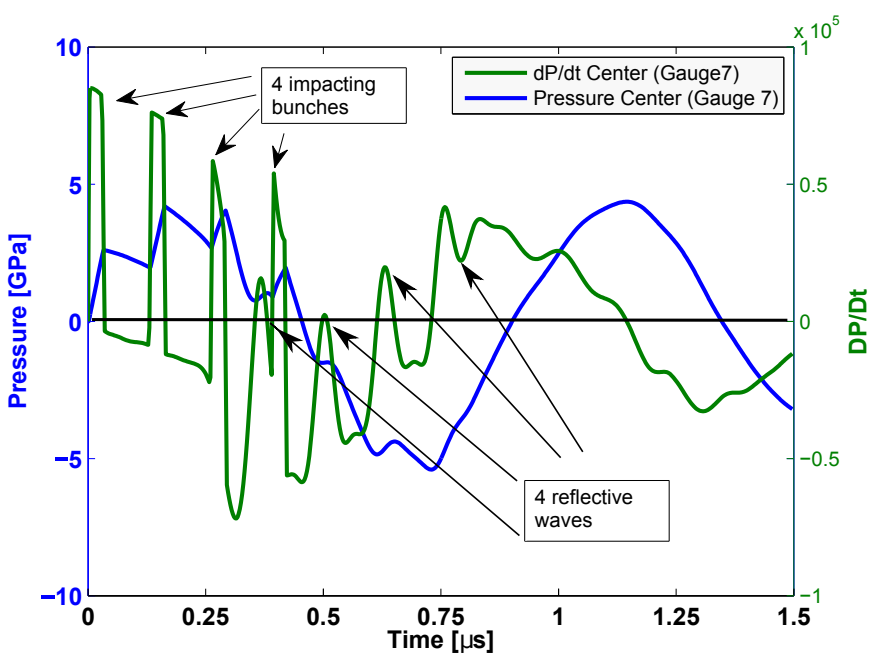

Figure 3.6: Pressure response (blue) and its time derivative (green) in the center of the target core (gauge 7 ) during the first $1.5 \mu$ s after proton pulse impact.

Another interesting phenomenon that deserves being explained in detail can be observed in figure 3.6, which shows the pressure response and its time derivative at the center of the target during the first $1.5 \mu \mathrm{s}$ of the transient. This plot includes therefore the four sudden rises of pressure corresponding to the four impacting bunches. The interesting phenomenon occurs at the end of each proton bunch impact, i.e. $105 \mathrm{~ns}, 210 \mathrm{~ns}, 315 \mathrm{~ns}$ and $420 \mathrm{~ns}$, and consists in an abrupt decrease of pressure as a consequence of the inertia after the sudden expansion of the material, as can be easily seen when plotting the pressure derivative (green line in figure 3.6). This end-of-bunch decrease of pressure is significantly relevant since it may be the cause of the strong compressive-to-tensile oscillation response present in the center of target. In particular, when this end-of-bunch decrease of pressure is in constructive interference with the natural radial wave.

In addition, the plot of the pressure derivative in figure 3.6 allows the observation of four small perturbation of the wave, delayed with respect the beginning of each single bunch impact. These perturbations are identified as reflective waves, 
and they are responsible of the small distortion of the fundamental radial wave observed during the first periods in figures 3.2 and 3.5. These reflective waves (which have a tensile nature) originate at the rod surface as a consequence of the local deposition of energy by the beam close the periphery. This boundary material is suddenly heated leading to the formation of compressive pressure, which is then rapidly reflected in the close surface and travels towards the center of the rod as a tensile wave, which amplitude is amplified when approaching the center due to the cylindrical geometry.

\subsection{Importance of the Proton Pulse Length on the Pressure Response}

The previous subsection allowed pointing out the existence of an end-of-bunch tensile wave taking place due to the inertia effects. In this section it is investigated whether these tensile waves can have an important influence on the overall response. For this reason, a parametric analysis assuming proton impacts with different pulse lengths has been carried out. The total energy applied for each of the scenarios is the same, with the only difference of the duration of the pulse. A $\mathrm{J}-\mathrm{C}$ strength model including plastic deformation of the material is used.

Figure 3.7 shows the pressure response in the center of the target assuming proton pulses with durations ranging from $0.1 \mu \mathrm{s}$ (blue) to $1.2 \mu \mathrm{s}$ (pink), in steps of $0.2 \mu \mathrm{s}$. As can be seen in the plot, the pressure magitude change completely depending on the length of the pulse, ranging from $+/-9 \mathrm{GPa}$ with $0.1 \mu \mathrm{s}$, pulse to +1 GPa only in compressive stress with $1 \mu \mathrm{s}$. The reason for this change is not only the obvious fact that the shorter the pulse the greater the power and inertia, but the influence of when the pulse is finishing. It can be observed in the plot that a tensile wave, which changes the trend of the pressure wave, always takes place at the end of the pulse. The effect of this end-of-pulse tensile wave differs significantly depending on whether the material is going to tensile or compressive state. When the material is going to tensile states, the end-of-pulse wave and radial wave produce a constructive interference leading to very large tensile stresses in the first period of the radial wave, which propagates to the rest of the transient pressure response. The latter effect can be easily noticed in figure 3.8; higher end-of-pulses pressure drops take place in the cases where pulses finish when $D P / D t<0$. This is the case of pulses with $0.43 \mu \mathrm{s}$ (as for the AD-Target), $0.6 \mu \mathrm{s}$ and $1.2 \mu \mathrm{s}$. On the other hand, in the pulses ending between $1 / 2$ and $3 / 4$ of the period of the radial wave (when the pressure is going to compressive states and therefore $D P / D t>0$ ), the end-of-pulse tensile wave is counteracted by the radial wave. This is the case of $0.8 \mu \mathrm{s}$ length and $1 \mu \mathrm{s}$ pulses in figures 3.7 and 3.8, where the tensile pressure reached is smaller than $-1 \mathrm{GPa}$. This phenomenon is even clearer when comparing the $1 \mu$ s pulse (yellow) with the $1.2 \mu$ s one. Higher tensile and compressive stresses are reached in the latter, even if the energy is deposited slower in this case. The 


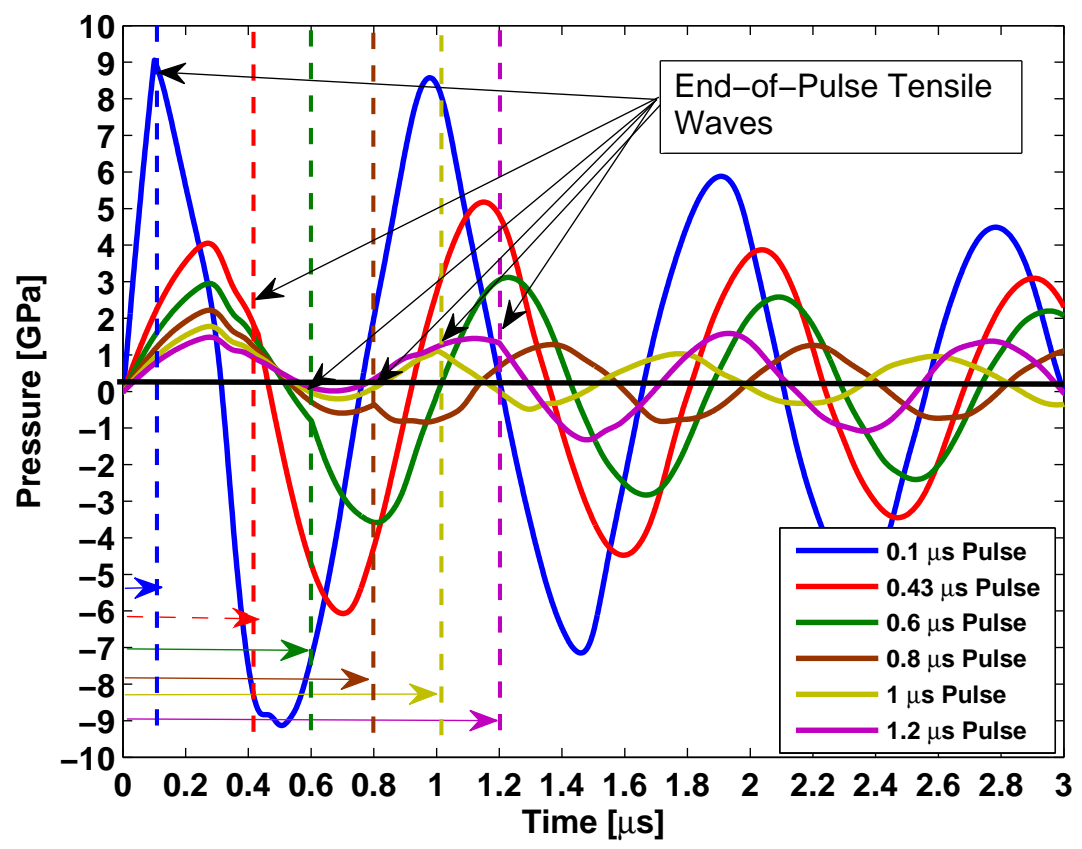

Figure 3.7: Parametric study showing the pressure response in the center of the target core for different proton pulse durations.

only exception to this response takes place with the pulse $0.1 \mu \mathrm{s}$, where due to the high inertia, the amplification of the end-of-pulse tensile wave takes place even if $D P / D t>0$.

In any case, a straightforward conclusion that can be drawn from this analysis is that a way to reduce the harmful tensile stresses reached in the AD-Target, given that the length of the pulses is strongly constrained by the PS and AD operation, would be to increase the diameter of the target core to 5-6 $\mathrm{mm}$ or even more, in order to increase the period of the radial wave and avoid its constructive interference with the end-of-pulse target. This diameter increase could however affect the antiproton re-absorption at the periphery of the target. This effect may not be very relevant with appropriate focusing but it needs to be demonstrated via Monte Carlo simulations where the global production, focusing, and selection of $\bar{p}$ are taken into account. 


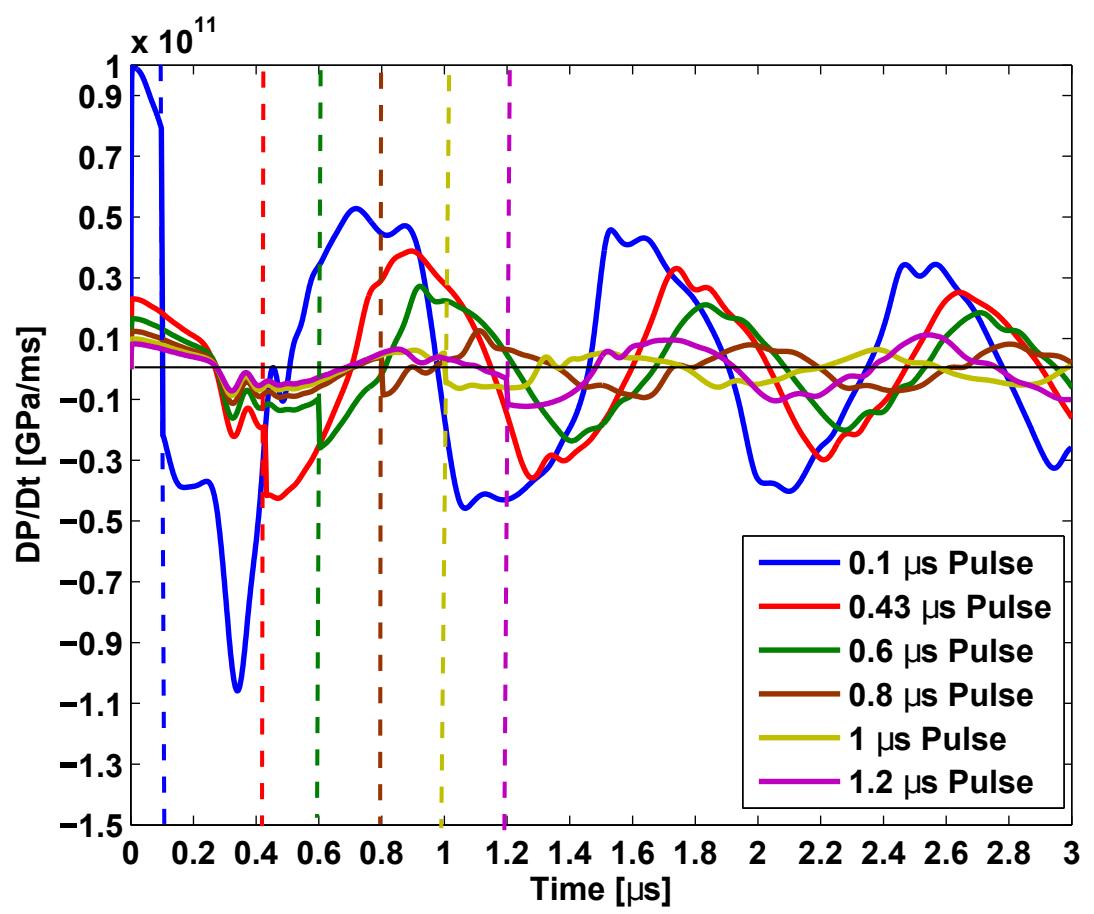

Figure 3.8: Same analysis as in Figure 3.7 but showing the pressure derivative for different pulse lengths. The influence of the end-of-pulse can be seen in the drop of the pressure derivative at the end of the pulses.

\subsection{Use of Core Cladding as a Strategy to Reduce Tensile Pressure in the Core}

As already introduced in section 2.8.3, a strategy to decrease the magnitude of the tensile pressure in the center of the target core could be the addition of an external cladding. This possibility is studied numerically in the present section by assuming three different scenarios:

- No cladding between the $3 \mathrm{~mm}$ diameter high density target core and the surrounding graphite matrix (Figure 2.10-a). This is the case of the current design of AD-Target.

- Copper cladding of $1 \mathrm{~mm}$ thickness (Figure 2.10-b).

- Tantalum cladding of $1 \mathrm{~mm}$ thickness (Figure 2.10-c). 
Figure 3.9 shows the pressure response at the center of the target core during the first $2 \mu$ s for the three scenarios of interest. As shown in the plot, the magnitude of the tensile pressure in the radial wave is significantly reduced when using copper and tantalum cladding. The reason for this reduction is probably a combination of 3 phenomena: (i) better impedance match between the core of tungsten and tantalum which causes a higher fraction of the energy of the pressure wave to be transmitted to the cladding instead of coming back as a tensile wave. (ii) the shift in the period of the radial wave due to the larger diameter as a consequence of the cladding, which could partially avoid the constructive interference between the end-of-pulse wave and the radial ones explained in the previous section. (iii) the plastic deformation in the tantalum or copper cladding material, which absorbs energy from the pressure wave, avoiding its reflection to the target core. As it can be observed in figure 3.9, a reduction by $44 \%$ in the maximum tensile pressure in the center of the target is obtained when using tantalum. This reduction can be important from the point of view of target survival and antiproton yield, especially if it is enough to reduce the tensile pressure below the spall strength of the material (which in tungsten was identified at 2.6 GPa [196]). Next section shows the significance of this reduction by the application of failure models.

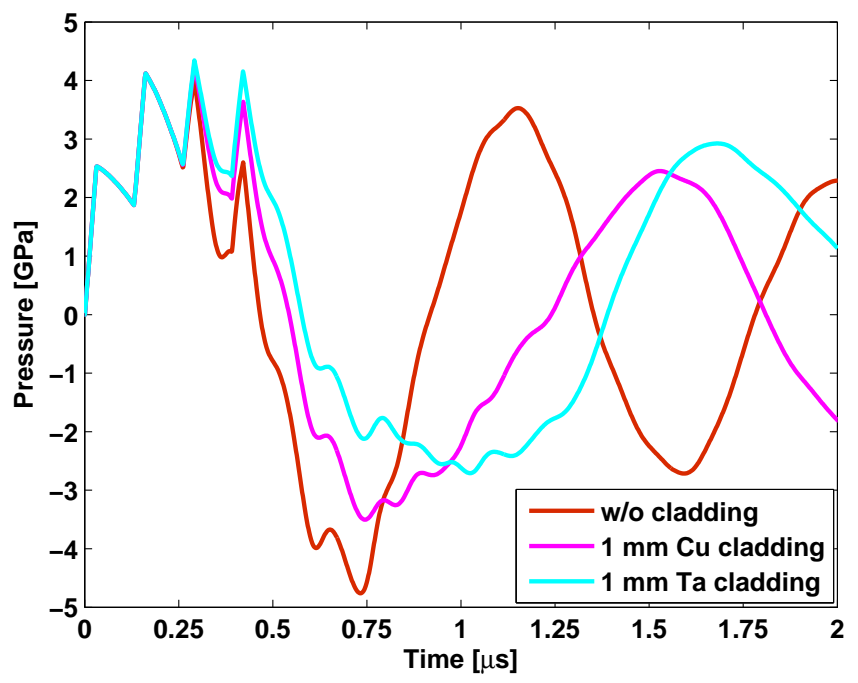

Figure 3.9: Pressure in the center of the target core for the three scenarios of study. Assuming a J-C strength model for the tungsten core and models considering plasticity in the cladding consistently with table 2.4. No failure models are considered. 


\subsection{Application of Failure Models to the Cases of Study}

In this section, the Minimum Hydrostatic Pressure failure model is included in the simulations, which is capable of predicting the possible material fracture wherever the tensile pressure threshold is exceeded in the target core due to the compressiveto-tensile wave taking place.

(a) Target w/o cladding

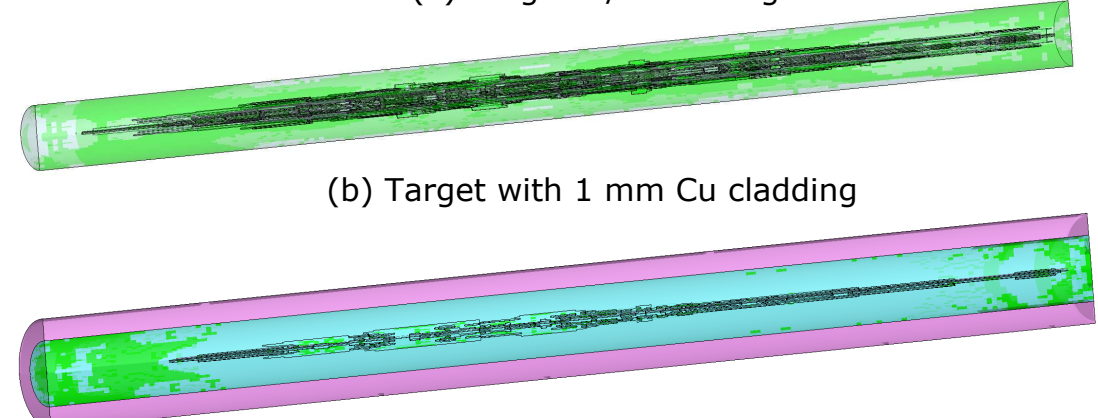

(c) Target with $1 \mathrm{~mm}$ Ta cladding

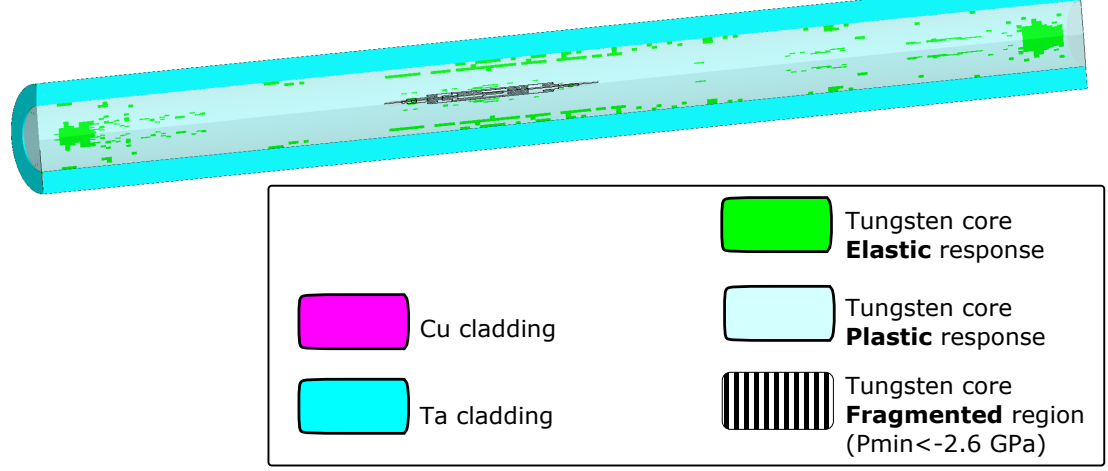

Figure 3.10: Target core material after a proton pulse impact considering minimum hydrostatic failure model in tungsten. $\left(P_{\min }=-2.6 G P a\right)$. The figure shows results for the three scenarios of study, demonstrating the efficiency of Ta cladding for reducing the internal core damage

Figure 3.10 shows the application of this failure model to the three cases of interest after a single pulse of the proton beam. As observed in the figure, for the case of a tungsten target without cladding, a large part of the volume of the target core is expected to be fragmented, leading to a reduction of antiproton production due to the loss of effective density. The use of tantalum cladding, on the other hand, could efficiently reduce the volume of the fragmented region. The calculations show that only $\sim 1 \%$ of the volume is fragmented with the Ta cladding solution 
in comparison with the non-cladded one. In addition, the figure shows how most of the material of the target core experiences plastic deformation. One of the main challenges is therefore to understand what would be the real effect of this plastic deformation and its damaging influence. Thus, the impact of subsequent pulses could certainly increase the fragmented volume presented here, but in any case it is shown that Ta cladding solution is much favorable than the non-cladded one. Copper cladding, in addition of being less effective, would not be feasible through HIP process due to the great mismatch in the coefficient of thermal expansion and melting point between the core and cladding materials. Another open point, not covered in this chapter, is the effect of plastic deformation at the core-cladding interface, which could lead to cladding detaching and therefore to reduce the improvement by the obtained cladding solution.

\subsection{Comparison between Tungsten and Iridium as Core Material}

As stated in section 2.7.2, all the calculations presented up to here consider tungsten as target core material instead of iridium. This material extrapolation was done due to the initial lack of strength and failure models of iridium in the literature. Nevertheless, in the context of this work, a dynamic characterization campaign was launched with the purpose of obtaining the strength model parameters of iridium, as explained in section 2.7.3. The output of this campaign was, among other things, to develop a J-C strength model applicable to iridium. In this section, this model is applied to simulations considering iridium as target core material. The results are compared with the simulations assuming tungsten performed in sections 3.2-3.5.

Figure 3.11 shows the pressure response of the iridium target core when considering a J-C strength model together with the response of tungsten already presented. In the figure it can be observed the presence of a compressive-to-tensile pressure wave, in the same way as it was taking place under the assumption of tungsten core. The end-of-bunch tensile waves are present as well. With Iridium the conditions seem to be even more extreme than with tungsten, reaching 6.5 GPa in compression and up to $8.3 \mathrm{GPa}$ in tension. This was expected due to the higher density of iridium (hence increasing the energy deposited by the beam), its higher thermal expansion coefficient, and its slightly higher bulk modulus. There is no information concerning spall strength or minimum hydrostatic pressure limit of iridium in the literature, in any case, the brittleness of iridium and the magnitude of tensile pressure reached suggest that a large volume of the core may be fragmented under these conditions, as observed for tungsten in figure 3.10-(a). 


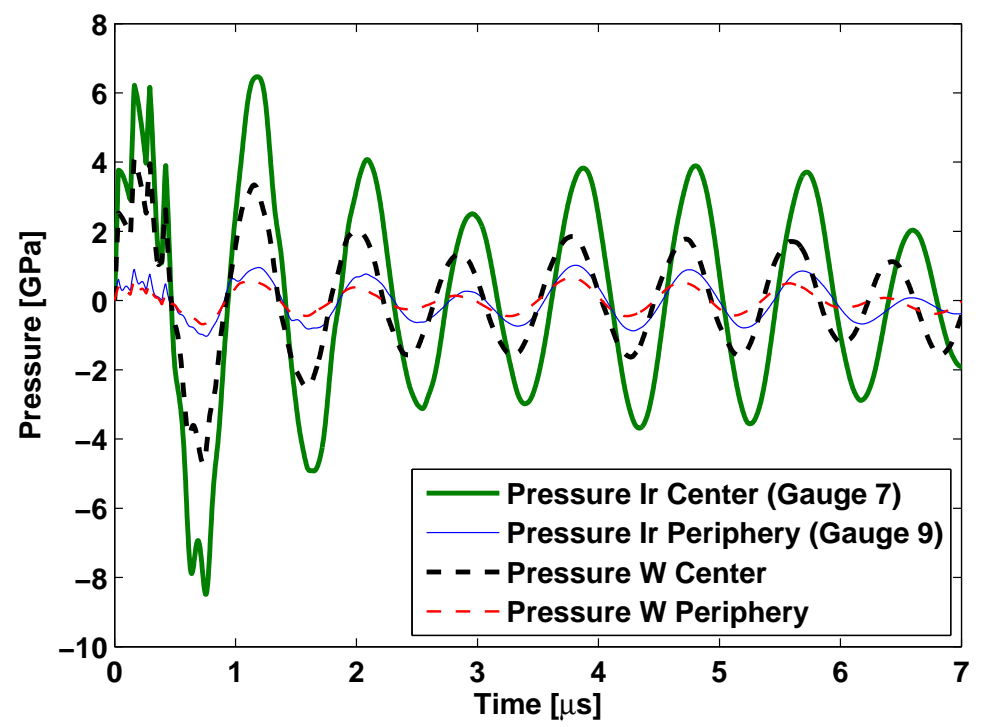

Figure 3.11: Pressure response at the center (gauge 7) and periphery (gauge 9) of the iridium target core after a $430 \mathrm{~ns}$ proton pulse impact. Response when considering tungsten is also included for comparison. This simulation considers J-C strength model for iridium, obtained from dynamic tests at DYNLab [67]. Note the higher magnitude of compressive and tensile pressures in comparison with the tungsten core.

\subsection{Response of the Graphite Matrix}

In the previous sections, several analyses have been dedicated to the pressure wave and material response of the target core, since the damage of this material would directly affect the $\bar{p}$ production. A question that may arise, however, is what is the response of the containing graphite matrix which is surrounding the target. Even if there is a great impedance difference between the target core and the graphite containing matrix, there will be always a fraction of the generated radial pressure wave in the core that will propagate through the core/matrix interface. This pressure wave cannot be neglected since, even if its magnitude can be much lower than the one in the core, the brittleness of graphite (which barely exhibits elastic deformation prior to fracture) may endanger its structural consistency.

With the purpose of understanding the propagation of the generated wave from the target core to the graphite matrix, figure 3.12-(a) shows the pressure response during the first $6 \mu$ s after the pulse impact at different radial positions in the graphite matrix (gauges 44, 45, 46 and 47 in 3.12-(b)). These gauges are placed in the longitudinal positions where the highest energies are deposited in the core 
and the highest pressures are reached (same longitudinal positions where gauges 7 and 9 were placed in the core and whose pressure response was shown in figures 3.2-3.11). The simulation presented here considered a J-C strength model in the tungsten, no failure in tungsten, and a visco-elastic strength model in graphite (without considering graphite failure either). The reason of considering tungsten as target core material, even if a J-C strength model was extracted for iridium, is the possibility of including also a failure model in the core later on (which is not available for iridium) to assess the potential fracture in graphite in a more realistic manner, taking also into account the fracture of the core.
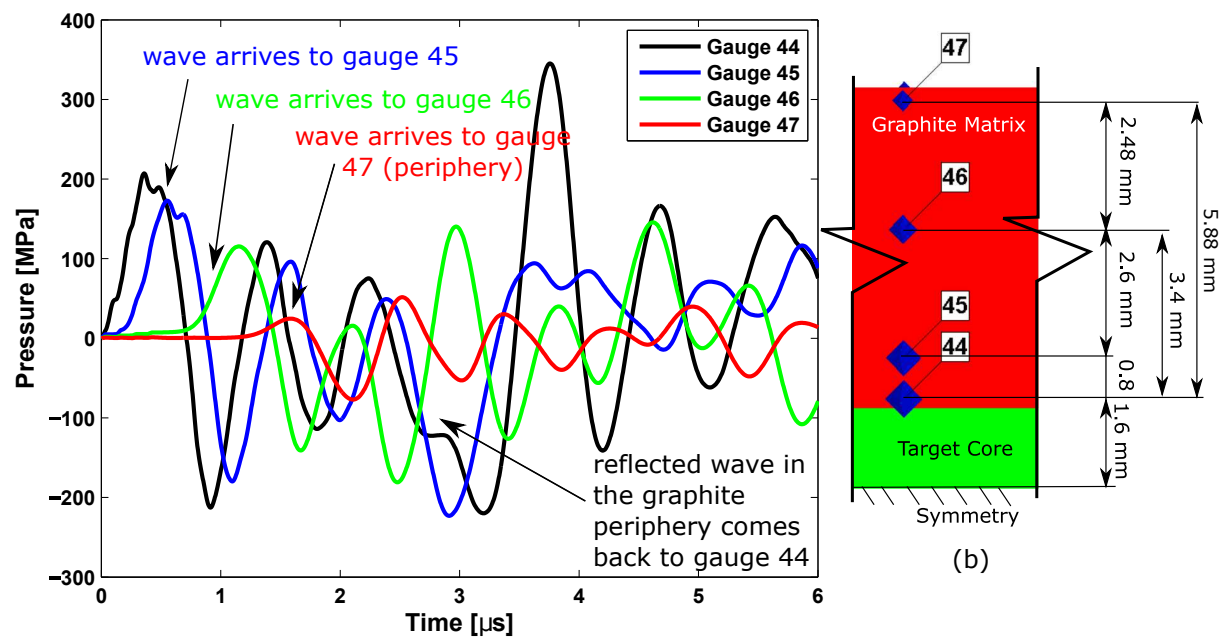

(b)

(a)

Figure 3.12: (a) Pressure response of the graphite matrix at different radial positions, from the vicinity of the target core interface (gauge 44) to the periphery (gauge 47). In the figure it is possible to observe the propagation through the graphite matrix of the radial oscillations coming from the core. (b) Section cut of the core-graphite matrix where the positions of the gauges whose response is shown in (a) is presented. The section cut corresponds to the longitudinal position where higher pressures and temperatures are reached.

The most interesting feature of figure 3.12 is that it is possible to observe the propagation of a wave from the core to the graphite periphery by its delay of arrival at the different gauges, placed at distances of $0.8,3.4$ and $5.88 \mathrm{~mm}$ respectively from the first gauge 44. This traveling wave consists of several oscillations of $\sim 0.8 \mu$ s duration, coming from the radial wave of the target core, and its front travels at approximate $3900 \mathrm{~m} / \mathrm{s}$ (speed of sound in graphite). The amplitude of these oscillations are reduced while they travel to the periphery due to the cylindrical geometry. In addition, it is possible to identify the reflection of this front at the periphery of the graphite matrix and the time when it reaches back the gauge 
44 by an abrupt change in the pressure trend there at $\sim 3 \mu$ s. This corresponds indeed with the necessary time to go over the $11.9 \mathrm{~mm}$ distance between the core interface + periphery + return to gauge 44 at $3900 \mathrm{~m} / \mathrm{s}$ (speed of sound in the material). After this reflection, there is a second peak of a considerably high amplitude of pressure in compression at the position of gauge 44, due to a constructive interference between the reflected waves and the ones that still arrive from the target core.

Looking in detail at figure 3.12, it can be seen that the pressure would reach magnitudes of $200 \mathrm{MPa}$ in compression and tension. Tensile strength of graphite varies significantly depending on the grade, supplier, pore quality, temperature, etc., due to the intrinsic brittleness and high anisotropy of this material. A common value found in the literature is around $30 \mathrm{MPa}$ [201], although it is possible to find scattered values of tensile strength covering from $10 \mathrm{MPa}$ [202] up to $69 \mathrm{MPa}$ [203]. Nevertheless, the stresses and tensile pressure reached in the graphite containing matrix according to 3.12-(a) are well above these limits, strongly suggesting that the graphite material may fracture as well, in addition to the core. For this reason, it is important to mention at this point that the plot of 3.12-(a) shows only a fictitious scenario in which the graphite materials does not fracture, useful only for illustrative purposes. The fracture of graphite would make that these stresses are never reached and the wave could not propagate as shown. In addition, in this simulation the fracture of the core was not considered. The core fracture would also significantly affect the generated radial wave and the kind of front that is propagated to the graphite matrix.

In order to clarify these aspects, an additional simulation was performed considering fracture in the tungsten core (as in figure 3.10) as well as introducing a failure model in graphite. The failure model selected for graphite was the Minimum Tensile Principal Strain, which was set at $6 \cdot 10^{-3}$. This value was extracted from spalling tests at room temperature using a SHPB setup with a long bar graphite specimen free at one end [197]. Figure 3.13 shows a the result of the simulation considering this configuration. As expected by the stresses shown in 3.12, a large volume of the containing matrix in contact with the target core fails due to the propagation of the wave coming from it. The fracture of the core is not shown in the figure due to post-processing limitations of AUTODYNR, even if this failure was taken into account by the simulation.

It is not straight-forward to predict all the consequences of this potential fracture in the graphite matrix. The most obvious implication would be an inappropriate cooling of the target core due to degradation of the core-graphite thermal contact, and its overheating when receiving successive pulses. In addition, fracture of graphite may be affecting the accuracy of the impact point of the incident proton beam since the target core (potentially fragmented as well) could slightly move in the free space left by the cracked graphite. In any case, it is important to mention that this calculation is somehow conservative in one aspect; the contact algorithm 


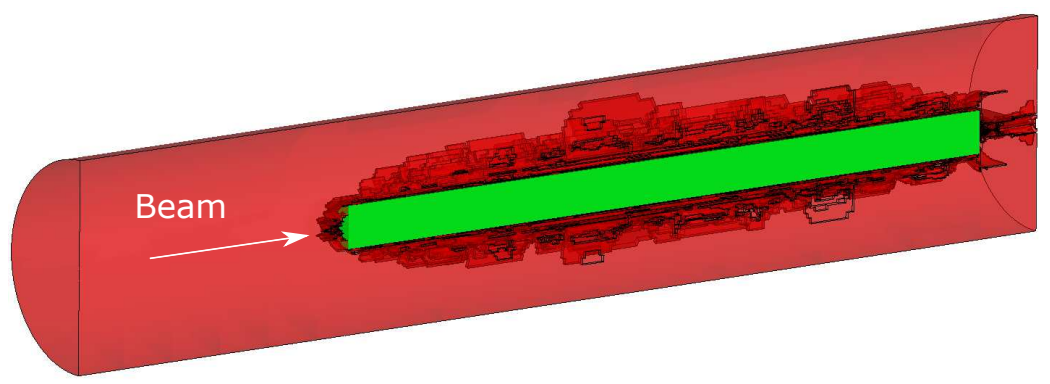

Figure 3.13: Fracture plot of the simulated state of the graphite matrix after the impact of a proton beam in the target core and subsequent propagation of the radial wave through the core-graphite interface by taking into account a failure model in both tungsten core and graphite matrix, although the plot only showed the fracture in the graphite.

implemented in AUTODYN@ between the core and the graphite matrix is assuming a perfectly bonded contact, which in reality is not the case. Nevertheless, the only way to confirm this result is by opening an already used target. This is complex due to its high radioactivity. Nevertheless, this possibility is under investigation, despite of not being in the scope of this thesis.

\subsection{Main Conclusions and Discussion of the Results}

In the present chapter, a detailed analysis of CERN antiproton decelerator target using hydrocodes has been carried out. This study emphasizes the extreme conditions reached in the $3 \mathrm{~mm}$ diameter, high density target core, in terms of adiabatic increase of temperature of more than $2000{ }^{\circ} \mathrm{C}$ each time the proton beam impacts the target and the subsequent pressure wave and dynamic response. The study does not cover the effect of further impact of consecutive pulses since their progressive damaging effect is subjected to the state of the material after the first pulse, propagating the already existing uncertainties in the calculations. In any case, it is obvious that further pulses will extent the level of target damage, although the generated pressure wave and its propagation through the material will be certainly different as the one presented here, due to the internal fragmentation of the target.

The understanding of the dynamic phenomena induced on the target core as a consequence of proton beam impact turns out to be fundamental in order to produce a robust design for the next 20-30 years of operation of the antiproton decelerator. Hydrocodes have been proven to be a powerful numerical tool, historically used for shock and impact physics, which are the only way to properly simulate this dynamic response. Their application on the target core is even more interesting, 
since its small and simple cylindrical geometry allows to easily note the propagation of the generated pressure waves and to understand fundamental aspects about them. Simulations starting from simple models assuming purely elastic material, while adding gradually more complex models which take into account plasticity or fracture, helped as well for this purpose. In this way, four main phenomena have been identified and described in this study:

I The identification of radial and longitudinal pressure waves (or modes) present in the target core with a period of $0.85 \mu$ s and $24 \mu$ s respectively. These frequency values were predicted analytically in sections 2.2.2 and 2.2.2 of Chapter 2, under certain assumptions. The radial wave has been found the most important and critical one, since the longitudinal is rapidly damped by the plastic deformation of the material. The radial wave, on the other hand, produces huge oscillating compressive-to-tensile pressure response in the center and periphery of the target core, probably leading to its internal fracture in the first oscillations, when reaching tensile stresses above the spall strength of the material.

II The existence and importance of the end-of-pulse tensile waves, consequence of the inertia of the heated material expansion. These tensile waves become especially important when they are in phase with the tensile part of the compressive-to-tensile radial wave described above. This is the case of the AD-Target core due to its small diameter of only $3 \mathrm{~mm}$. Avoiding this constructive interference will be a way to reduce this limiting phenomenon.

III The possibility of using a 1-mm thick cladding surrounding the $3-\mathrm{mm}$ diameter target core as a way to decrease the acoustic impedance mismatch between the target core and graphite containing matrix, with the aim of decreasing the tensile pressure reached in the center of the high density target core. Simulations show that a reduction of up to $44 \%$ in the maximum tensile pressure can be achieved by the addition of tantalum cladding.

IV Results suggest that the generated radial pressure wave in the target core is propagated to the containing graphite matrix. This results in very high stresses there, which may endanger its structural state due to the brittleness of graphite. Simulations suggest that a large fraction of the graphite may be fractured close to the core-matrix interface, even only after a single proton beam impact.

Nevertheless, one must be aware of the intrinsic limitations of these simulations, which have been performed using material models based on simple assumptions and experimentally fitted as the J-C model. These models are applied here to a much more complex case than a simple mechanical impact test. The nature of the 
load itself, the variation of temperature and strain rates in this short time, will lead to several phenomena which cannot be considered by the code, like possible re-crystallization processes (if there is enough time for them), presence of preexisting defects, material mechanical hysteresis or radiation damage. In addition to these phenomena, the influence of the partial extrapolation of the used strength models (extracted at temperatures up to $1200{ }^{\circ} \mathrm{C}$ and applied here up to $2000{ }^{\circ} \mathrm{C}$ ) on the simulations accuracy must be taken into account. This extrapolation had to be carried out due to the lack of experimental data in the literature at the extreme regime reached.

For this reason, the analysis in the current chapter only represents the numerical approach of the antiproton target redesign process presented in this thesis. An experiment in the HiRadMat testing facility at CERN (presented in Chapters 4, 5 and 6) has been carried out to complete the numerical work introduced here. 



\section{Chapter 4}

\section{The HRMT27 Experiment: Recreating Equivalent Conditions as in the AD-Target}

\subsection{Introduction and Motivation of the Experiment}

The application of advanced numerical tools, such as hydrocodes, to the resolution of the extreme dynamic conditions reached in the AD-Target has been proven to be a powerful methodology to reduce the uncertainties of the response of the target core material and to bring important insights of its structural state. Simulations including studies of beam parameters influence such as the pulse length or possible strategies to try to mitigate the damage in the target core were also carried out. However, these numerical analyses, shown in Chapters 2 and 3, suffer from intrinsic limitations, like most of the numerical tools, that must be taken into account and require that numerical simulations and experimental work go hand by hand, even more when these simulations are applied to such complex problems as the one under study.

The main conclusion from Chapter 3 is that the radial pressure wave generated as a consequence of the sudden deposition of energy of the incident proton beam in the target material exposes it to very high tensile stresses, well above its strength limit and therefore endangering its structural state. Estimations of the extend of this damage have been done by the application of failure models, such as the Minimum Hydrostatic Pressure. This model predicts that a large fraction of the target core may fail, assuming that the core is made of tungsten and that internal 
cracking takes place when a tensile pressure of -2.6 GPa (obtained experimentally in laser induced free surface spall in polycrystalline tungsten [196]) is exceeded. Tungsten was considered as core material since values of spall strength for iridium have not been found in the literature. Nevertheless, there are still several key questions of this problem that need to be understood:

i What really happens to the material exposed to this extreme wave phenomena and high tensile pressure load.

ii Which are the main or the different mechanisms of failure.

iii What is the impact of this failure on the material effective density and therefore antiproton production (due to the reduction of beam-target material interaction).

iv Is it there other high density material that behaves better than the ones numerically studied?

Additionally, it is important to note that numerical simulations can only go as far as the constitutive models of the materials used. As already mentioned in section 2.6, hydrocodes have been developed and applied historically for projectile impacts and therefore the availability of material strength models (most of them obtained experimentally by Hopkinson Bar Tests) is limited to regimes of relatively low temperatures and strain rates, always well below $2000{ }^{\circ} \mathrm{C}$ and $10^{4} s^{-1}$ as the ones reached in the core. No constitutive strength model of any of the material candidates exists for the regime of application and therefore the results given by the simulations are subjected to the extrapolation of the material models. Furthermore, the failure mechanisms and limits need to be identified experimentally in order to be properly implemented in the code. The Minimum Hydrostatic Pressure failure model could be a good first approximation; however, this tensile pressure limit is obtained normally by spall impact or laser tests on free surfaces, in which unfortunately the load characteristics and material conditions are different than the case of study, where these enormous temperatures and tensile pressures are reached in the bulk material. It is clear that an experiment is necessary.

In that sense, it is hard to imagine any other experimental way than a proton pulse impact to produce inside a large amount of bulk material the fast and high temperature gradient and tensile pressures reached in the AD-Target. Previous experimental works have been done using the approach of applying high and fast current electric pulses (up to $9.2 \mathrm{kA}$ ) in W, Mo, and Ta wires [79]. However, the sudden rise of temperature associated with these electric pulses was in the order to $200{ }^{\circ} \mathrm{C}$ and stresses of the order of $400 \mathrm{MPa}$, which are much lower than the case of study. Another approach could be laser beam impact, which is able to deposit large amount of energy in a material in a very short amount of time. However, this deposition will be limited to the material surface instead of the bulk 


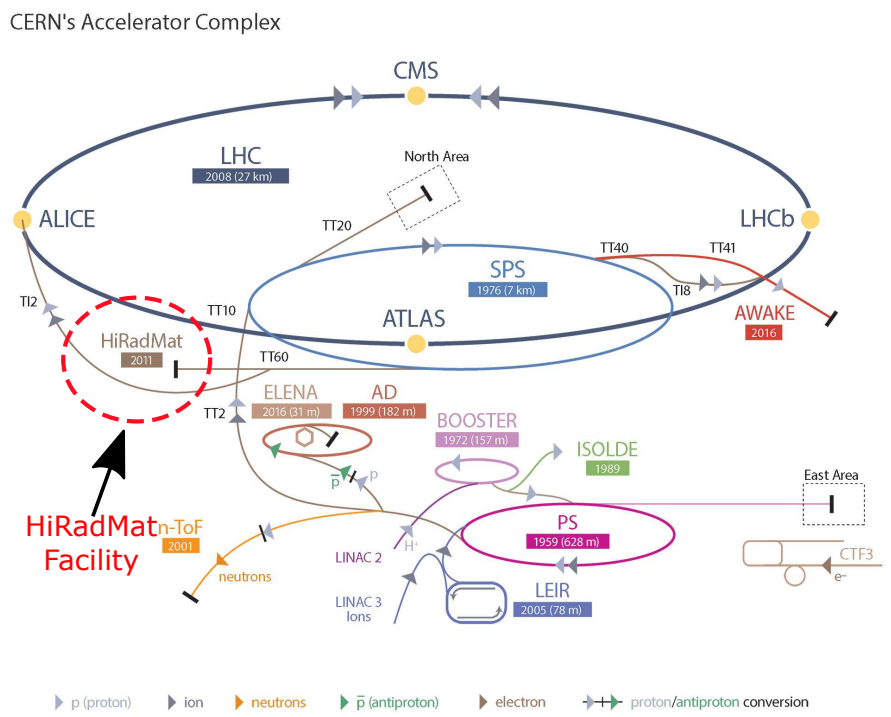

Figure 4.1: Scheme of the CERN Accelerator Complex where the HiRadMat Facility is highlighted. This facility uses beam of $440 \mathrm{GeV} / \mathrm{c}$ momentum from the SPS. An important part of the design of the HRMT27 experiment was devoted to find the way to create equivalent conditions as reached in the $\mathrm{AD}$-Target, where the incident proton beam consist of $26 \mathrm{GeV} / \mathrm{c}$ instead of $440 \mathrm{GeV} / \mathrm{c}$.

as it is taking place in the target core. For these previous reasons, it was concluded that an experiment using direct proton beam impacts is the only way to assess the level of accuracy of the strength model extrapolations done in Chapter 3 and to identify real failure mechanisms and material limits at the reached conditions.

In this context, the HiRadMat facility [13] at CERN was identified as a very appropriate place for such kind of test. This facility is in operation since 2012, using one of the extraction lines of the SPS as it is shown in figure 4.1. In this way, it is able to provide intense proton beams from the SPS with a momentum of $440 \mathrm{GeV} / \mathrm{c}$ and a wide flexibility of intensities and beam optic parameters, making it very suitable for testing accelerator equipments.

The dedicated experiment presented in this thesis was called HRMT27-RodTargand took place in November 2015. The experiment intended to impact high intensity and energy proton pulses onto thin rods (8 $\mathrm{mm}$ diameter and $140 \mathrm{~mm}$ length) of high-density materials such as iridium, tungsten, molybdenum and tantalum among others, recreating analogous conditions as reached in the AD-Target in a controlled environment. The present chapter includes a summary of all the activi- 
ties related to the experiment, including its objectives, detailed design to overcome the involved engineering challenges, and operational procedures for its execution.

\subsection{Definition of Goals of the Experiment}

After the definition of the motivation for the experiment recently introduced, the scientific objectives associated to this motivation are presented. These objectives can be summarized in two main goals.

I Cross check and validation of the numerical hydrocodes employed to simulate the material response of the antiproton target core: This objective is carried out by measuring online the response of each of the sample rods when impacted by the proton beam, such as temperature and vibration (displacement and velocity) on their surface. These measurements could be directly compared with the numerical predictions and, in this way, assess the level of accuracy of the extrapolation of the strength models. In addition, the targets are subjected to a stepwise increase of intensity during the test in order to record information at different conditions and regimes.

II Gaining experimental insights, reducing uncertainties in targets response, and assessing the selection of the future antiproton target material: If different high-Z candidate materials such as W, Ir, Mo or Ta are irradiated and exposed to equivalent conditions as they would reach in the AD-Target, quantitative and qualitative comparison between their response will certainly give important indications of their performance. Furthermore, the experiment strives to find the limits of the materials of interest and to identify mechanisms of failure at these conditions. The stepwise increase of impacted proton beam intensity is also intended for this purpose, allowing to infer the conditions at which important changes in the material are taking place by observing the change in the response of the external surface of the targets (described in detail in section 4.4.1). In addition, some targets of the materials of interest are irradiated only up to intermediate intensities in order to study the progressive material damage during the post irradiation examinations (PIEs).

It is important to mention that the experiment was especially focused on the dynamic response of the target core. In that sense, it was not the scope of the experiment to prototype the future design of the target but to obtain information of the wave phenomena and core material behavior from a fundamental point of view. Therefore, some aspects such as the response of the graphite matrix and its possible fracture were not included in the experiment, since it was considered that the addition of a graphite matrix surrounding the experimental targets would have complicated the access of the instrumentation to the core, as well as adding new uncertainties in the simulations vs experimental comparisons. 


\subsection{Involved Challenges}

The experiment aimed at accurately impacting high energy and intense $440 \mathrm{GeV} / \mathrm{c}$ proton beams onto very thin targets in a completely controlled environment, recreating unprecedented extreme conditions in their bulk materials and, at the same time, recording phenomena that take place in the order of microseconds. This task involves several technical challenges that are summarized in the following sections.

\subsubsection{Creating Equivalent Conditions in the HiRadMat Targets as in the AD-Target}

The HiRadMat facility employs proton beam from the SPS at $440 \mathrm{GeV} / \mathrm{c}$ while the AD-Target receives beam from the PS at $26 \mathrm{GeV} / \mathrm{c}$. Not only differences in the kinetic energy exist but also in parameters such as beam size, number of bunches and pulse length. Fortunately, the SPS can provide beam to the HiRadMat facility with flexibility regarding these parameters (but not in the energy). In order to recreate the same conditions in HiRadMat as in the AD-Target, it is necessary to adjust the impacted intensity, beam size, pulse length and target geometry. The objective of this process is to reach a similar profile of deposited energy density and hence achieving an analogous peak of temperature in the HiRadMat targets as it would be reached in the antiproton target. In addition, the shape and timing of the deposited energy and the interaction of the generated pressure wave with the target geometry has to be in such a way that a compressive-to-tensile pressure radial response as the one presented in figure 3.5 of section 3.3 takes place, including the end-of-pulse constructive interference described in section 3.4. In this study it was shown by numerical simulations that the high level of tensile pressure reached in the core of the AD-Target takes place because the pulse burst finishes when the natural radial wave is already exposing the material to tensile states (second half of its period), leading to a constructive interference between this natural wave and the end-of-pulse one. For this reason, in the HiRadMat experiment the pulse length must be at least half or the natural radial period of the targets. Finally, the design of the targets must be done in such a manner that the conditions at their periphery are not too extreme in terms of reached velocities and temperatures, so they are below the instrumentation technical limitations. Sections 4.4 and 4.4 .3 present the solutions to these compromises and challenges. 


\subsubsection{Challenges in terms of Accuracy and Beam Monitoring}

In order to recreate analogous conditions in the HiRadMat targets as reached in the AD-Target and obtain the corresponding radial wave, it is fundamental that the beam hits in the center of the targets with a very high level of accuracy. This task is not always straightforward, as the targets are very thin and are placed in a mobile structure necessary for positioning each of them on the incoming proton beam trajectory. In addition, possible "jitter" or pulse-to-pulse instabilities of the proton beam could take place, complicating even more this task. It is for this reason that a very accurate beam position monitoring system was necessary in order to detect and correct the position of the targets in case of misalignment.

\subsubsection{Instrumentation Selection and Configuration}

The third challenge involves the selection of instrumentation. This instrumentation needed to be compatible with a harsh environment while providing very accurate data. These conditions can be summarized in terms of temperature variations (target surface could reach up to $900{ }^{\circ} \mathrm{C}$ ), velocities and inertia forces on the target surfaces $\left(40 \mathrm{~m} / \mathrm{s}\right.$ and $\left.10^{8} \mathrm{~m} / \mathrm{s}^{2}\right)$ and very high levels of prompt radiation produced due to the high density materials used as targets. The high levels of prompt radiation reached excluded the use of any active electronic device close to the targets. It is for these reasons that mainly optical passive instrumentation was used in the experiment, which provides the advantage of not being in contact with the targets surface (as it would happen for example with strain gauges) and the possibility of using only passive optical heads close to them, allowing to transfer the recorded signal by optic fiber to a radiation shielded bunker where the electronics and DAQs (Data Acquisition Systems) are placed. More details of the solution used are given in section 4.4.3. In addition, the instrumentation needed to have very high acquisition rates (above $\mathrm{MHz}$ ) for being capable of recording the desired waves since the dynamic response of the target would take place in the order of microseconds. Finally, the instrumentation had to present a large flexibility in a wide range of measurement conditions, as the stepwise increase of impacted intensity and the difference of density of the irradiated targets would make changes in the variables to measure of more than one order of magnitude, depending on the impacted intensity and the corresponding target during the experimental beam setup phase. 


\subsubsection{Activation of Targets}

During the experiment, high density materials received a relatively high number of proton pulses (a total of $8.44 \cdot 10^{13}$ protons cumulative for all the targets). The interaction of the proton beam with the nuclei of these materials produces unstable isotopes and therefore activate the targets. The design of the experiment had to be done in a way that these targets could be easily and quickly extracted and dismounted after the experiment in order to reduce radiation exposure. For this reason, the experiment was designed following the guidance of remote handling and maintenance in radiation areas at CERN [204], which is inspired in the ITER Remote Handling Code of Practice. The applied measures following these guidelines include minimum size and type of fasteners (so they can be easily engaged by robots to remove the targets), modular configuration including particular types of connections which would optimize the time for disassembling the experimental setup, and materials prone to low radio-activation such as aluminum for the sample holder. 


\subsection{Design of the Experiment}

\subsubsection{Design of the Targets and Beam Parameters}

The targets geometry and beam parameter selection was the first step in the experiment design. As already mentioned in the section 4.3.1, the design of the experimental targets had to satisfy the compromise of a set of variables including the recreation of analogous conditions as reached in AD-Target, being compatible with the characteristics of the HiRadMat proton beam and, at the same time, producing a response at their surface within the instrumentation limitations. A long iterative and optimization process involving targets geometry, beam parameters, and instrumentation selection had to be carried out for this purpose. The solutions presented in the next two subsections (4.4.1 and 4.4.1) as well as in the one describing the selected instrumentation (section 4.4.3) are the results of this iterative process.

\section{Recreating equivalent conditions as in the AD-Target}

The first aspect deduced during the initiation of the target design process was the need of scaling. A $3 \mathrm{~mm}$ diameter rod as in the real AD-Target would not have been practical for several reasons: First of all, the fixed momentum of $440 \mathrm{GeV} / \mathrm{c}$ of the HiRadMat proton beam required to blow up the incident beam to achieve energy depositions and temperatures similar to the ones of the AD-Target. This required defocusing of the HiRadMat beam can be seen figure 4.2 in which the maximum energy density deposited as function of the target length is plot for the $26 \mathrm{GeV} / \mathrm{c}$ beam of the AD-Target (red) and the $440 \mathrm{GeV} / \mathrm{c}$ of HiRadMat (blue) considering a beam spot size of $1.5 \times 1.5 \mathrm{~mm}$ at $1 \sigma$ for the latter. The plot shows that, with the selected intensity, a 3 times larger beam spot size in HiRadMat beam than in the AD-Target is required for reaching an equivalent maximum energy density deposition. A more focused beam in HiRadMat would have demanded to decrease further its intensity, which is not convenient since SPS operation requires minimum beam intensities to run. The figure 4.2 also shows a shift of $\sim 40 \mathrm{~mm}$ in the maximum deposited energy density between the two types of beam. This is due to the fact that the incident proton beam of HiRadMat has an energy of $440 \mathrm{GeV}$ instead of the $26 \mathrm{GeV}$ of the AD-Target. This high energy relativistic beam is able to travel for a longer distance through the high density material without barely interacting with it before it starts depositing larger amounts of energy. This difference is intrinsic to the beam and cannot be avoided. Nevertheless, it is not expected that this difference would affect the generated radial pressure wave.

The requirement of defocusing of the HiRadMat beam just described led to the conclusion that the rod diameter had to be increased too in order to get an equiva- 


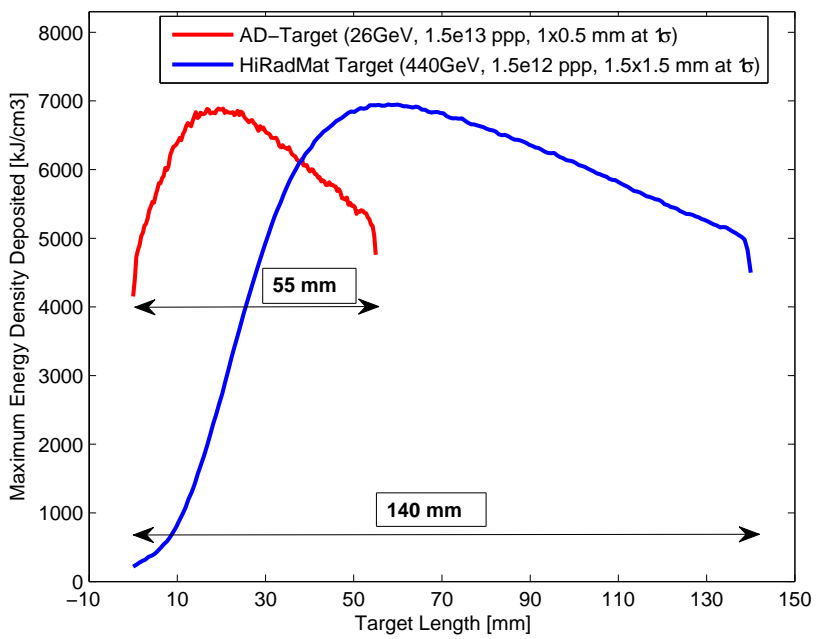

Figure 4.2: Plot of the maximum energy density deposited as function of the target length for the AD-Target beam $(26 \mathrm{GeV} / \mathrm{c})$ and HiRadMat beam $(440 \mathrm{GeV} / \mathrm{c})$. The length 0 in the plot corresponds to the upstream side of the targets.

lent beam/target-radius ratio. In addition, very thin rods would have complicated even more the mechanical precision requirements and would have also been more sensible to beam trajectory instabilities. Finally, it can be shown by simulations that doing the target diameter smaller would increase the velocities reached on the surface, well above the instrumentation limitation of $24 \mathrm{~m} / \mathrm{s}$ and $2 \mathrm{~m} / \mathrm{s}$ of the LDVs (Laser Doppler Vibrometer) and interferometers respectively (shown in section 4.4.3).

After several iterations of simulations, a rod of $8 \mathrm{~mm}$ diameter as shown in figure 4.3 was found as a compromise. This diameter was deemed enough to produce a tensile-to-compressive pressure wave while keeping the surface velocity below $24 \mathrm{~m} / \mathrm{s}$ at the point of measurement. The length of the target was also selected conditioned by the fact that a $440 \mathrm{GeV} / \mathrm{c}$ proton beam would have a significant different longitudinal profile of deposited energy than the one of a $26 \mathrm{GeV} / \mathrm{c}$ beam. The density of the irradiated materials played an extra role as well in the length selection in order to have an equivalent longitudinal profile of deposited energy in all the targets. Based on that, a length of $140 \mathrm{~mm}$ for the targets of tungsten and iridium was selected, $170 \mathrm{~mm}$ for the tantalum, and $240 \mathrm{~mm}$ for the molybdenum. Figure 4.4 shows a comparison between the geometry of AD-Target core and the reference geometry of experimental targets (140 mm length). 


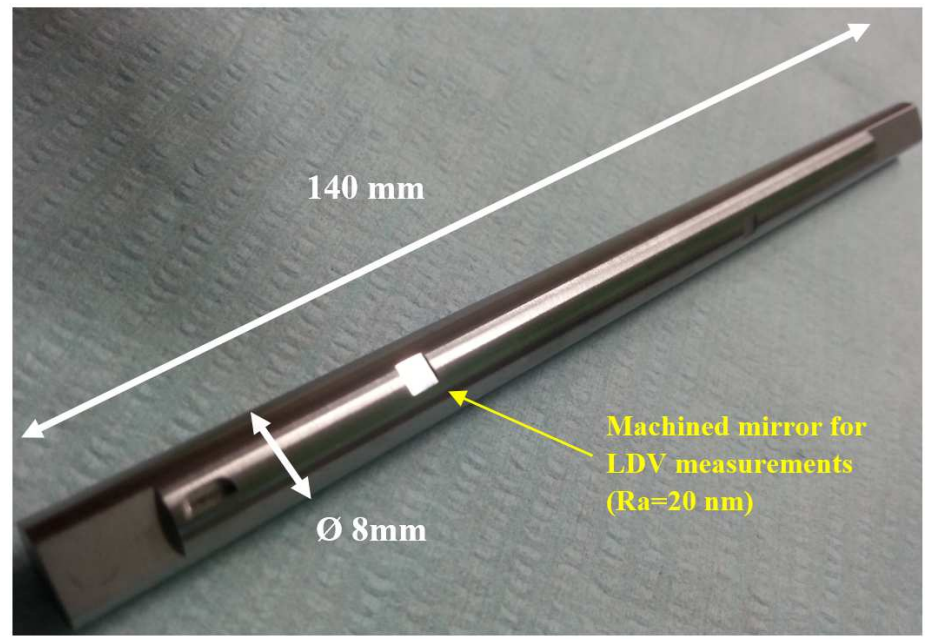

Figure 4.3: Picture of a tungsten target showing one of the multiple mirrors machined on its surface, where the LDV was pointed in order to measure its vibration when impacted by the proton beam.

\section{Core of the AD-Target}

\section{HRMT-27 Experimental Target}

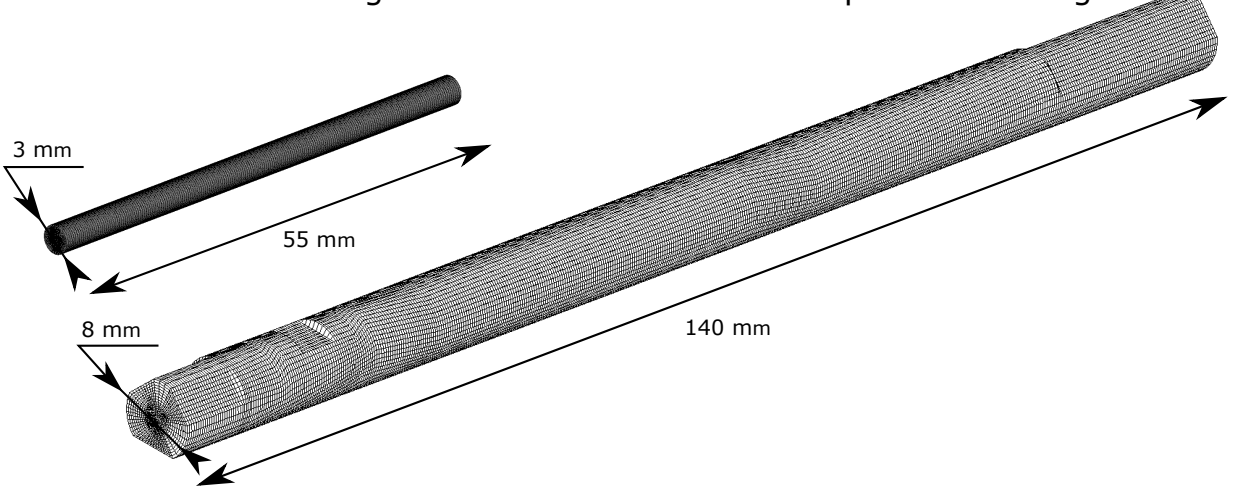

Figure 4.4: Mesh of the AD-Target and HRMT27 target used in the Hydrocode simulations carried out for the design of the experimental targets. The scaling of the experimental targets, which are significantly larger, can be observed in the figure. 
Together with this geometry, the beam parameters such as intensity, beam size and pulse length were selected in order to achieve an equivalent adiabatic rise of temperature in the bulk material of around $2000{ }^{\circ} \mathrm{C}$ and a compressive-to-tensile pressure wave reaching several GPa. In that sense, the selection of the pulse length of HiradMat was fundamental to have this compressive-to-tensile response (as demonstrated in section 3.4 of Chapter 3). For this reason, the length of the HiradMat pulse was set to $0.9 \mu \mathrm{s}$, composed of 36 bunches, spaced by $25 \mathrm{~ns}$. This pulse has a duration of approximately half of the period of the generated radial wave, achieving the end-of-pulse tensile wave and radial wave resonance amplification taking place also in the AD-Target.

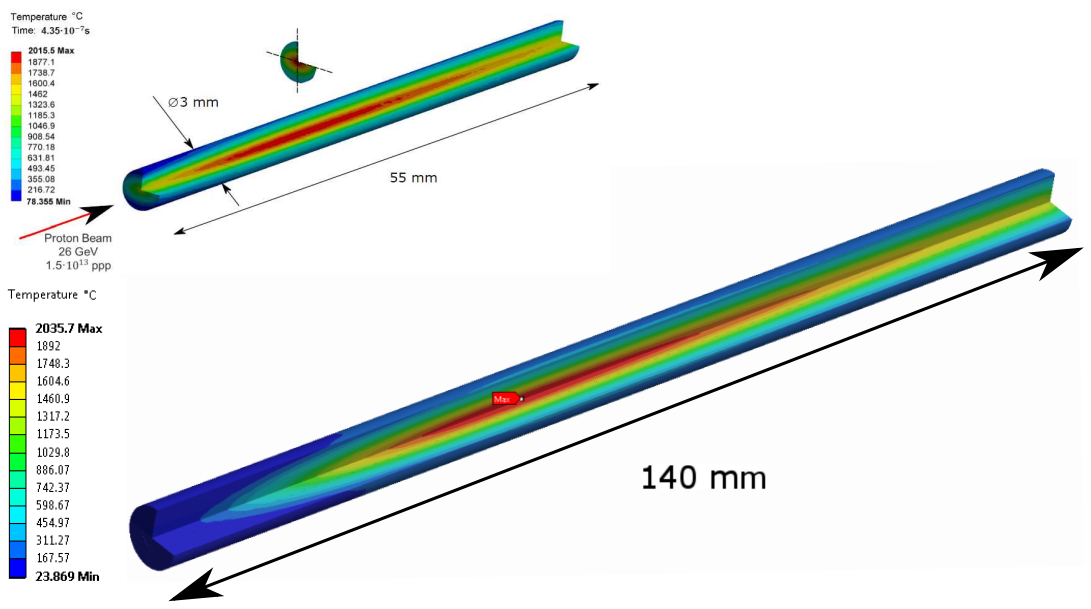

Figure 4.5: Comparison between estimated temperature reached in the AD-Target (up) and in the designed HiRadMat targets (down), assuming tungsten in both targets.

Figure 4.5 shows a comparison between the temperature profile reached in the AD-Target (up) and HiRadMat target (down) considering a proton beam impact in the latter target of $1.5 \cdot 10^{12} \mathrm{ppp}$ with a beam size of $1.5 \times 1.5 \mathrm{~mm}$ at $1 \sigma$. This comparison is carried out assuming tungsten as target material for both rods. It can be noticed in the figure how with this beam parameters the adiabatic rise of temperature in both targets is quite similar, reaching a maximum around $2000{ }^{\circ} \mathrm{C}$ in the bulk material and a temperature gradient from the center to the periphery close to $1800{ }^{\circ} \mathrm{C}$. This comparison of reached temperature in the HRMT-target vs AD-Target core, even if is done assuming tungsten as material, can be perfectly extrapolated to every other tested material (iridium, molybdenum, tantalum...), i.e, with these beam parameters the conditions reached in the experiment would be quite similar to the ones taking place in the AD-Target, no matters the material. This is justified by the fact that with the selected beam, the peak of energy deposited in a specific material would be the same in both geometric configurations. 


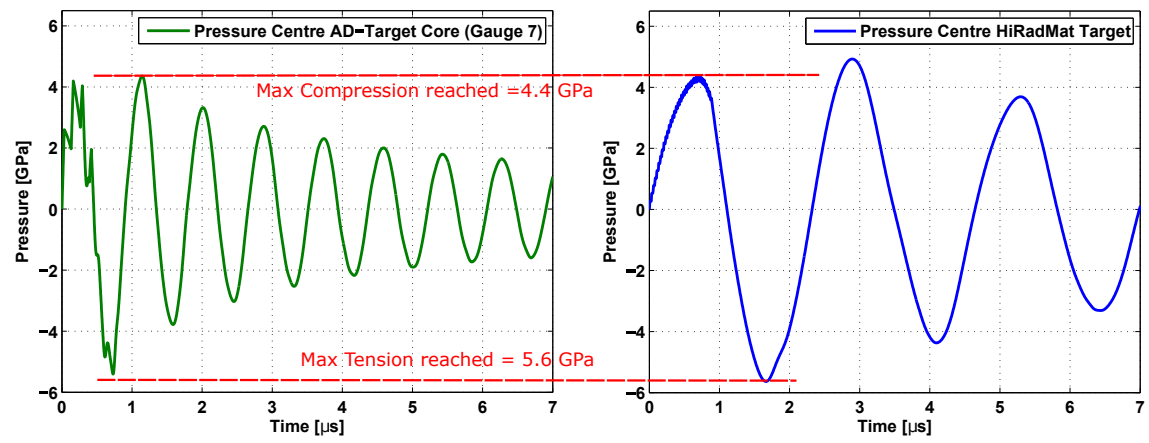

Figure 4.6: Comparison of the simulated pressure response in centre of the AD-Target core (left) and the HRMT27 experimental target (right)

The main difference that can be observed between the temperature profile reached in the AD-Target core and the HiRadMat target takes place in the first 2-3 cm upstream the HRMT-experimental target. As can be seen in the figure, temperatures there are relatively low (blue) in comparison with the upstream part of the AD-Target core. As anticipated previously, this is due to the fact that the incident proton beam of HiRadMat has an energy of $440 \mathrm{GeV}$ instead of the $26 \mathrm{GeV}$ of the AD-Target.

Figure 4.6 shows a comparison of the simulated pressure response in AD-Target core (left) and the HRMT27 experimental target (right), consequence of the sudden deposition of energy of the $1.5 \cdot 10^{12} \mathrm{ppp}, 0.9 \mu \mathrm{s}$ duration, beam of HiRadMat. These simulations are considering a J-C strength model and do not consider failure of the material (so the waves can be easily compared). As can be seen, a radial compressive-to-tensile pressure wave also takes place in the HiRadMat target due to the end-of-pulse tensile wave amplification phenomenon, achieving equivalent conditions as in the AD-Target. A pressure of $4.4 \mathrm{GPa}$ is expected in compression and $-5.6 \mathrm{GPa}$ in tension. The main difference is in the radial period of the wave produced HiRadMat, which is expected to be around $\sim 2.3 \mu$ s instead of the $\sim 0.8 \mu$ s of the AD-Target core, due to the larger rod diameter of the experimental target. 
Figure 4.8: Estimation of failure of a tungsten HRMT27 target at conditions reached in the AD-Target core assuming $\mathrm{W}$ and a Minimum Hydrostatic Pressure failure model with a failure threshold of $2.6 \mathrm{GPa}$.

\section{Expected Velocity Response in the Targets Surface}

Figure 4.7 (a) and (b) shows the expected surface velocity of the experimental target periphery at the longitudinal region where the largest amount of energy is deposited. In figure (a) the simulation does not consider a failure model, while in figure (b) the Minimum Hydrostatic Pressure failure model for tungsten is considered. As can be seen in the picture, velocities above $40 \mathrm{~m} / \mathrm{s}$ are expected in both cases. This velocity is well above the maximum range of measurement of best LDVs in the market, which is $24 \mathrm{~m} / \mathrm{s}$. Higher velocities with high acquisition rates may be measured by VISAR (Velocity Interferometer System for any Reflector) but their commercialization and importation are quite restricted due to its military application. The solution found to overcome this instrument limitation is described in next section (4.4.1).

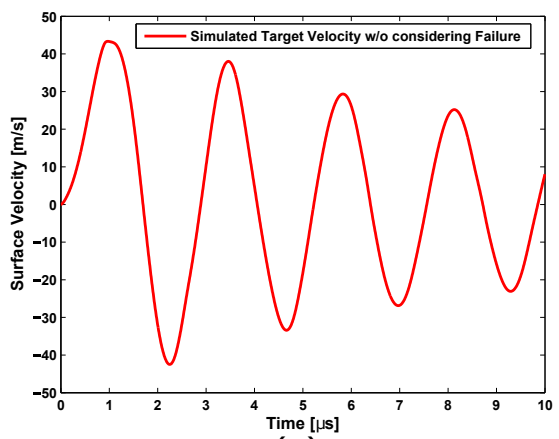

(a)

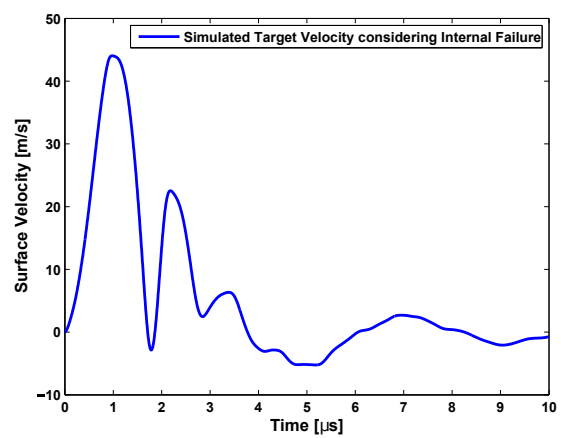

(b)

Figure 4.7: Comparison of the simulated surface velocity when reaching AD-Target conditions in the experimental targets. (a) Without considering a failure model. (b) Considering a failure model

Nevertheless, the interesting feature of the comparison between figure 4.7-(a) and (b) is the evident change in the velocity response when failure is considered. In the latter, the internal fracture would take place in the first negative oscillation of the pressure wave. Figure 4.8 shows the simulated fracture of the target at these conditions. As a consequence of this fracture, the radial wave is clearly damped 
after a few oscillations as shown in figure 4.7-(b), presenting as well a period reduction since the effective radial distance of travel of the wave is reduced due to the portion of cracked material in the inner regions of the target. This change in the response and distortion of the radial wave will be useful for interpretations purposes during the experiment, in particular to help identifying when, and at which conditions, internal fracture of the irradiated targets is taking place.

\section{Selection of Points of Measurement and Mirror Roughness Requirements}

Even if the conditions reached in the AD-Target were recreated with the experimental targets of the geometry presented in the previous section, there is an additional requirement that had to be fulfilled, i.e. to be able to actually measure the vibrations of the targets. The velocities reached in some points of the target surface are so high, (well above $40 \mathrm{~m} / \mathrm{s}$ ) that in order to avoid saturation of the instruments, the selection of points of measurement had to be done very carefully. Figure 4.9 shows the measurement points selected after several iterations and cross-checks with simulations. As can be seen in the figure, all the points of measurement of velocity and displacement are placed close to the upstream extremity of the target, where velocities are considerably lower (below the $24 \mathrm{~m} / \mathrm{s}$ instrumentation limitation) than in the longitudinal centre.

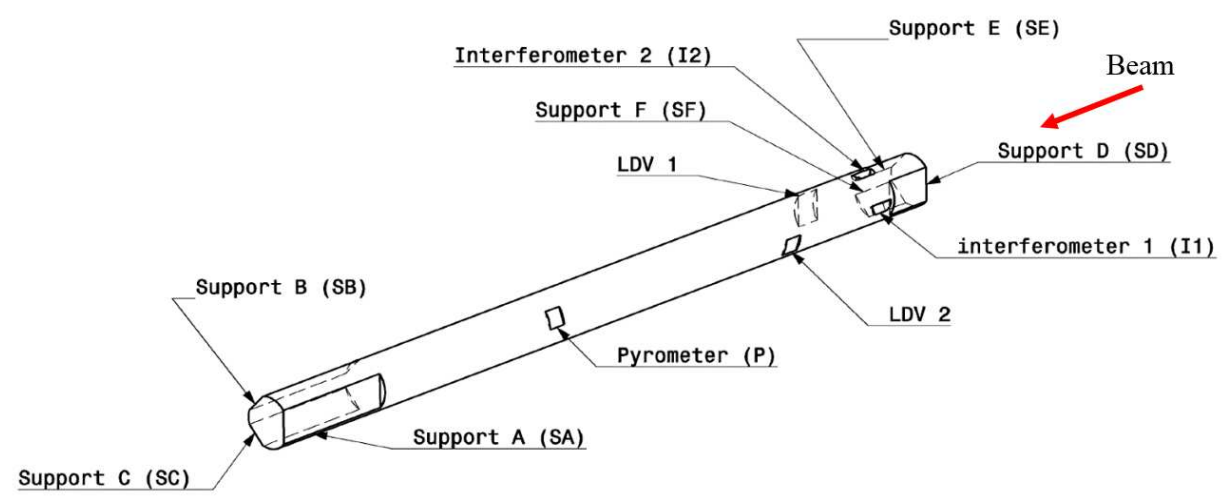

Figure 4.9: Isometric view of the $140 \mathrm{~mm}$ long targets, indicating the points of measurement of the different optical instruments used in the experiment.

As observed in table 4.1, the interferometer has a velocity limitation of $2 \mathrm{~m} / \mathrm{s}$ instead of the $24 \mathrm{~m} / \mathrm{s}$ of the LDVs. For this reason, an extra effort was put in the design of the targets in order to make them compatible with this instrument limitation. The solution of reducing a velocity from $40 \mathrm{~m} / \mathrm{s}$ to $2 \mathrm{~m} / \mathrm{s}$ is presented in Figure 4.10-(b). This solution consisted in machining small slots on the target 


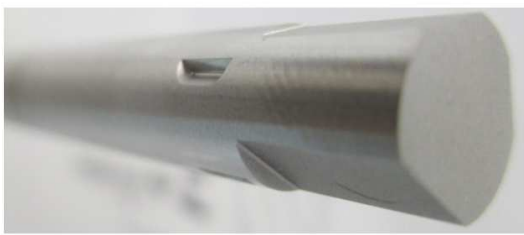

(a)

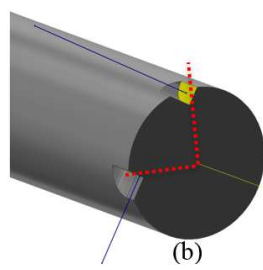

(b)

Figure 4.10: (a) Upstream end of an iridium target, including the small slot for the interferometer measurements. (b) Cross-sectioned view of the target in the section where the inclined surface for interferometer measurement were machined.

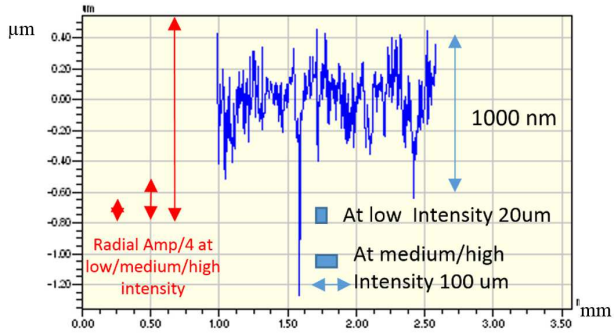

Figure 4.11: Roughness profile of one of the target mirrors with a Ra of $0.13 \mu \mathrm{m}$. As can be seen in the figure, this roughness would not be acceptable for the experiment since the expected lateral oscillations of the surface - up to $20 \mu \mathrm{m}$ (blue arrows)-could create variations on the peak-to-valley above $0.8 \mu \mathrm{m}$, i.e. larger than the desired amplitude to measure when irradiating with low intensity pulses.

in such a way that the surface of measurement forms a $30^{\circ}$ angle with the radius of the cross section. It can be shown by simulations that a velocity projection of the radial wave takes place, resulting a speed reduction in this inclined surface of more than one order of magnitude in comparison to the external surface velocity. These inclined surfaces were machined in the targets by EDM (electrical discharge machining), giving the hardness and machining difficulties of the materials of interest (iridium, pure tungsten, molybdenum...). The V-shape support of the sample holder allowed the fixation of the targets in a precise and accurate angular position, since these small surfaces had to be perfectly perpendicular to the interferometer optical head, as it is shown in figure 4.18.

Another crucial requirement for the targets specification was the mirror roughness. It was observed in the simulations that, due to the tri-axial nature of the material expansion and mode excitation, the exact points where the optical instruments were aiming at would also move and oscillate in the longitudinal direction. This would result in a longitudinal displacement of the point of measurement, with a frequency corresponding to the one of the radial wave. This circumstance brought 


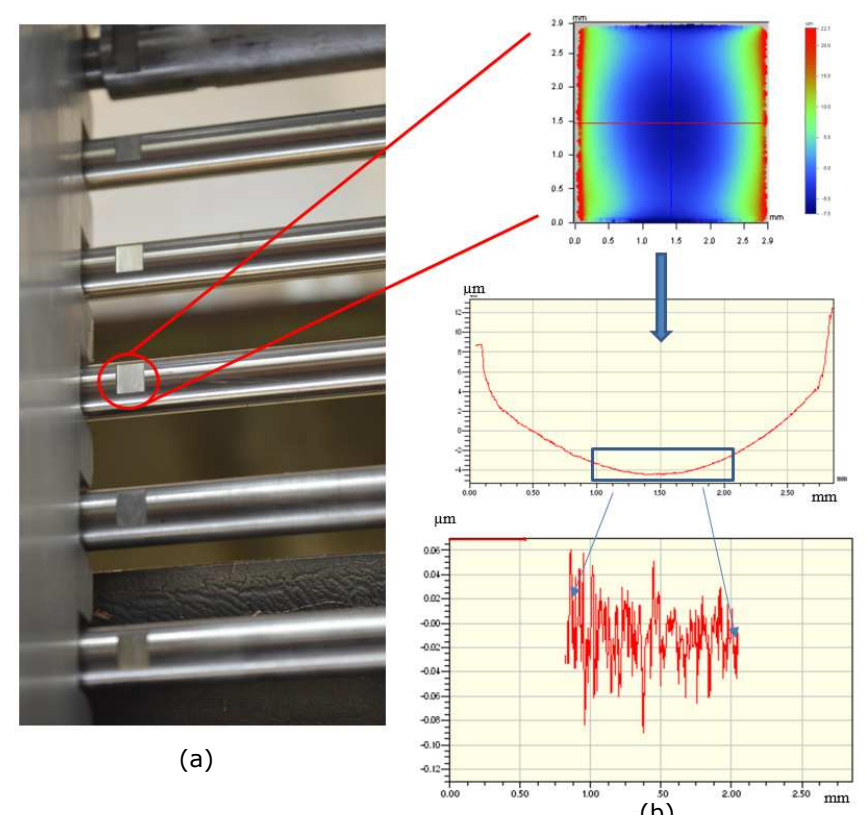

(b)

Figure 4.12: (a) Detailed picture of the upstream part of the targets installed in the sample holder, including the mirrors used for the measurements of the Polytec $\mathbb{R}$ LDV. (b) Results of measured roughness on the mirror of a tungsten target after the secondary polishing process using microdiamond powders. $\mathrm{Ra}=20 \mathrm{~nm}$ was achieved with a peakto-valey value of $120 \mathrm{~nm}$, overcoming the concerns on influence of roughness in the measurements.

up the concern that, if the surface roughness is in the order of magnitude of radial wave to be measured (which could have an amplitude of approximately $0.4 \mu \mathrm{m}$ in the low intensity pulses) and the point of measurement changes, it could provoke that only the surface roughness is spuriously measured instead of the desired radial displacement. Figure 4.11 illustrates this problem.

A way to minimise these uncertainties would be to reduce surface roughness as much as possible. This roughness specification was set to $\mathrm{Ra}=0.05 \mu \mathrm{m}$ based on the expected amplitude of the radial wave at low intensity impacts. It was not trivial to achieve this quality due to the hardness of the target materials and the lack of experience in polishing them by most suppliers, since most of these materials are extremely uncommon (such as iridium) and there is virtually no practical application which requires these levels of surface quality. Finally, and after having tried several strategies, such as electro polishing, it was found that this level of surface quality could be achieved by fine polishing techniques using microdiamonds powders. Figure 4.12 shows the mirror quality after this polishing 
process for one of the tungsten targets. Roughness of $\mathrm{Ra}=20 \mathrm{~nm}$ was achieved, one order of magnitude lower than the before-polishing presented in Figure 4.11.

\subsubsection{General Layout}

The experiment was designed in a modular way in order to facilitate the mounting and dismounting procedure. Figure 4.13 shows a CAD model of the full assembly of the experiment while in figure 4.13 a picture of the experiment installed in the HiRadMat facility (TNC line) is presented. As can be seen in the figure, there are three main parts in the setup:

I Standard HiRadMat table: This table is provided by the HiRadMat facility in order to make all the experiments compatible with the available space and multiple electrical and cooling connections of the facility. The table provides a quick plug-in system under its base in a way that it can be almost remotely installed by a crane in the experimental position.

II Experiment support tables with horizontal and vertical degrees of freedom: The experiment is equipped with a motorized mobile table with two degrees of freedom: vertical and horizontal. These degrees of freedom were used to move the vacuum tank for the alignment of the targets with the incoming beam, using beam position monitors BPKG/BTV (Beam Pick-up Button Position [205]/Beam TV [206]) which were attached to the tank. These two beam position systems together can provide online information about the beam parameters, such as position (with $0.1 \mathrm{~mm}$ accuracy), intensity and size. More details of the alignment procedure of the experiment is given in section 4.6.1. The movement of these tables and position control was done by means of step motors and LVDT (Linear Variable Differential Transformer) systems.

III Vacuum tank and attached BPKG/BTV system: The vacuum tank of $610 \mathrm{~mm}$ height, $500 \mathrm{~mm}$ length and $420 \mathrm{~mm}$ width was placed attached to the mobile tables. The tank included; two visual portals made with radiation resistant glass to avoid their darkening during the experiment [207], two $0.254 \mathrm{~mm}$ thickness Be windows for inlet/outlet of the proton beam and five feedthroughs for the in-tank instrumentation. Upstream to the tank, the BPKG/BTV assembly was attached (as shown in figure 4.17) and accurately aligned with the target on beam position.

Inside the vacuum tank, 13 targets were placed in an aluminium sample holder which moved vertically during the experiment to put each corresponding target in the on-beam position as shown in figure 4.15. The vertical movement of the sample holder was driven by a step motor which was placed in the top of the tank, together with a LVDT system, which accurately determined the vertical 


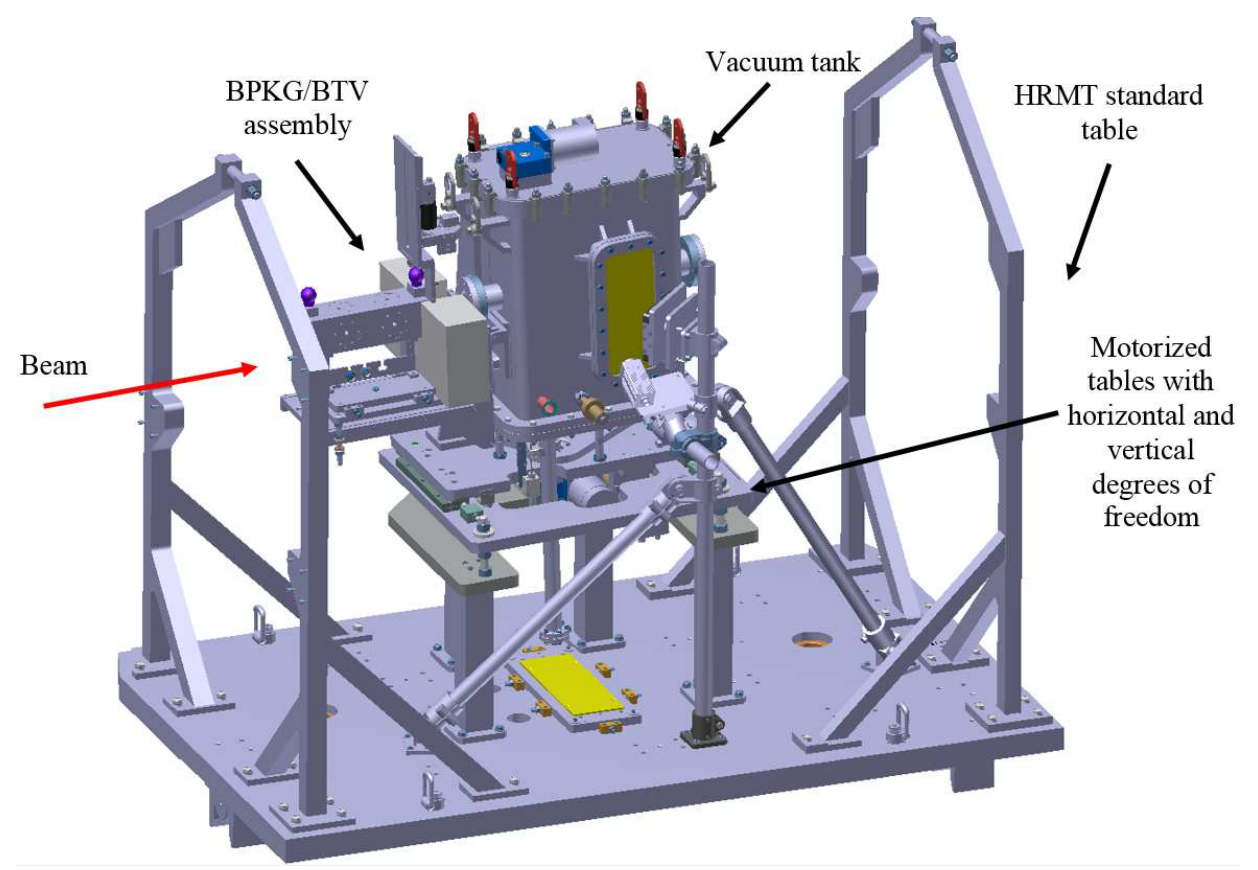

Figure 4.13: 3D model of the full HRMT27 Experiment.

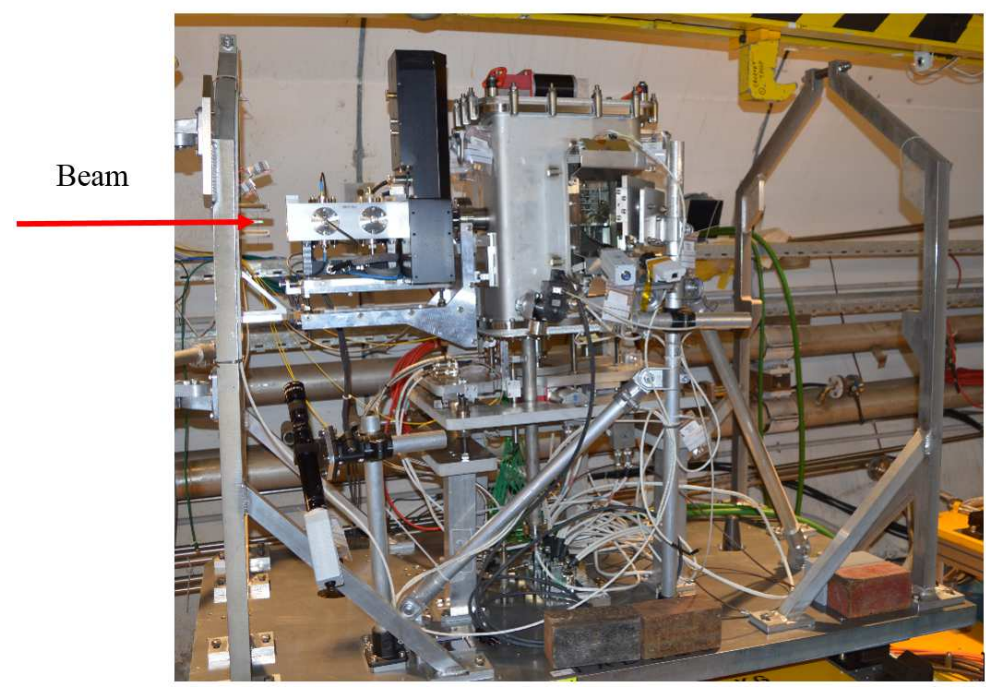

Figure 4.14: Picture of the HRMT27-RodTarg- experiment installed in the tunnel of HiRadMat. 


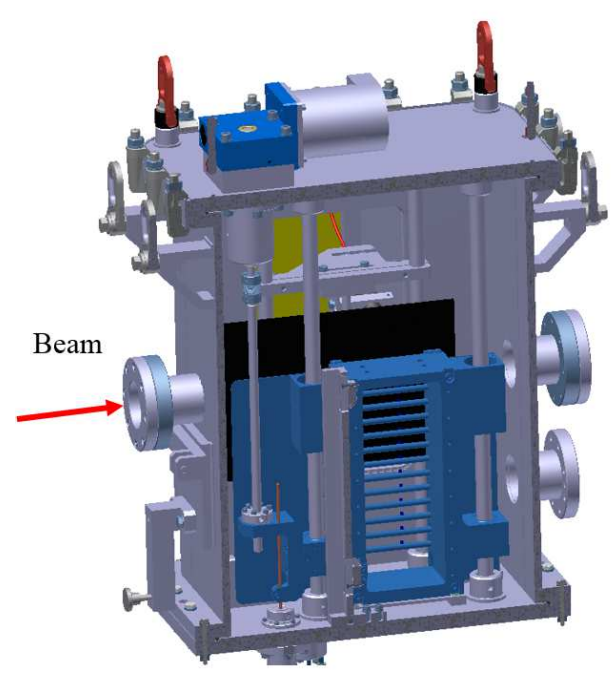

(a)

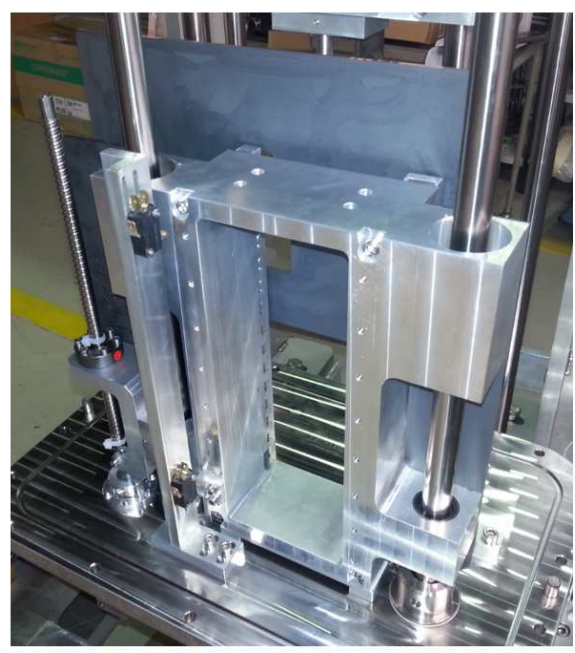

(b)

Figure 4.15: (a) Inner view of the vacuum tank, including the mobile sample holder made of aluminium (blue).(b) Picture of the sample holder during the assembly.

position. An empty space in the sample holder was added to allow the passage of pulses without interacting with any target during the alignment procedure or other possible configurations. In addition, this sample holder was used in the dismounting phase of the experiment as it could be easily extracted from the top of the tank. On the sample holder, each of the target rods was clamped by small V-shape graphite supports, which pressed the rods by means of springs as shown in figure 4.16. These supports were fundamental for the proper angular positioning of the targets and alignment with the optical instruments, which were pointing at very small mirrors machined on their surfaces.

Inside the vacuum tank, four optical heads were placed, pointing directly at the target on-beam position and the mentioned mirrors machined on its surface. Two of these heads corresponded to an interferometer, one to a Laser Doppler Vibrometer (LDV) and the other one to a pyrometer. These optical heads were placed behind a water-cooled copper screen (black plate in figure 4.15-(a)) which was installed between the instrumentation and the targets. The screen had small holes to allow the direct view of the targets by the instrumentation. The purpose of this screen was to protect the instrumentation of possible fragments of the targets during the experiment, which could damage the optical heads. In addition, it was designed to help cooling down the targets by radiation heat transfer as it was very close to the target surfaces and was covered by a graphite painting that increased its emissivity. 


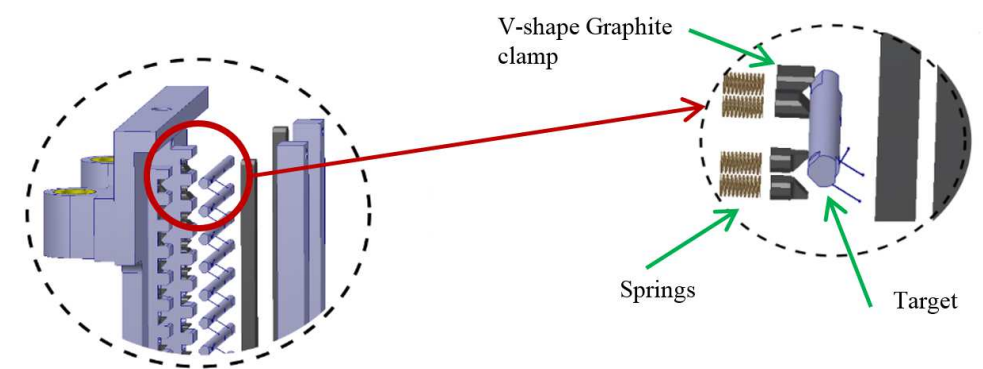

Figure 4.16: Detailed view of the of the V-shape clamping system of the targets in the sample holder. These $\mathrm{V}$-shaped clamps were made of graphite and pressed the rods using small springs.

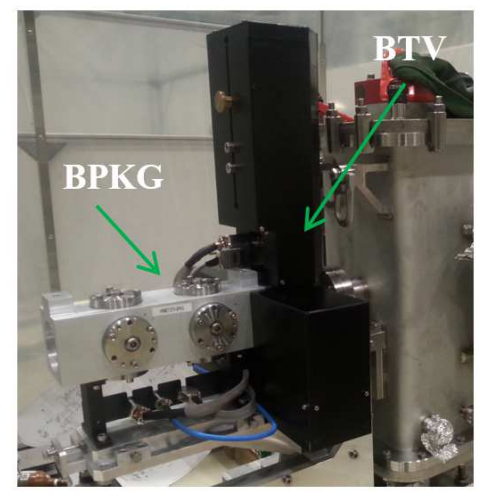

(a)

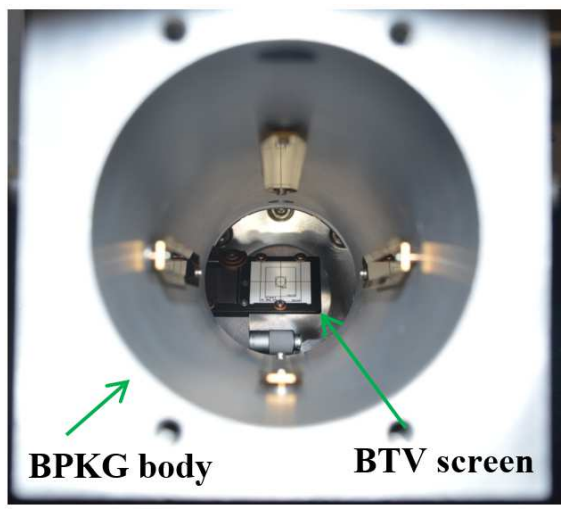

(b)

Figure 4.17: (a) Picture of the BPKG/BTV assembly used to monitor the position of the beam during the experiment. (b) Frontal picture of the BPKG where is possible to see the screen of the BTV system.

Outside the tank, and attached to the standard HiRadMat table, a supporting structure was placed. This structure supported a radiation hard camera and a HD camera, which pointed at the interior of the tank through one of the visual portals as shown in figure 4.20-(b). The lighting inside the tank was implemented by LEDs from PowerStart series, featuring 4 Oslon SSL Square ThinGand LEDs [208] and externally attached to the window of the tank. In addition, the external structure supported a mirror which permitted to have an optical path between the target which is on-beam position and a LDV placed in the bunker of an adjacent tunnel. 


\subsubsection{Instrumentation Selection}

As introduced in previous section 4.3.3, the selection of the instrumentation was one of the most challenging tasks of the experiment. This instrumentation, if placed close to the targets, had to work subjected to very high prompt radiation (coming from the high energetic nuclear interactions and fast decays in the first instants after the beam-target impact). In addition, in case of being attached to the targets, would have to be subjected to huge temperature variations (target surface could reach up to $900{ }^{\circ} \mathrm{C}$ ) during the recording time, which had to be in the order of microseconds to catch the phenomena of interest. These specific challenges discouraged the use of strain gauges attached to the targets surface since there were important uncertainties regarding its recording under prompt radiation (which is normally associated to a strong electromagnetic-pulse) and temperature dependence (they work based on changes in electric resistance, which is very temperature-dependent). Finally, velocities and inertia forces on the target surface could lead to its detachments. For these reasons it was thought that optical instrumentation (completely contactless to the targets surfaces) such as LDVs, interferometers and pyrometers was the best possible solution.

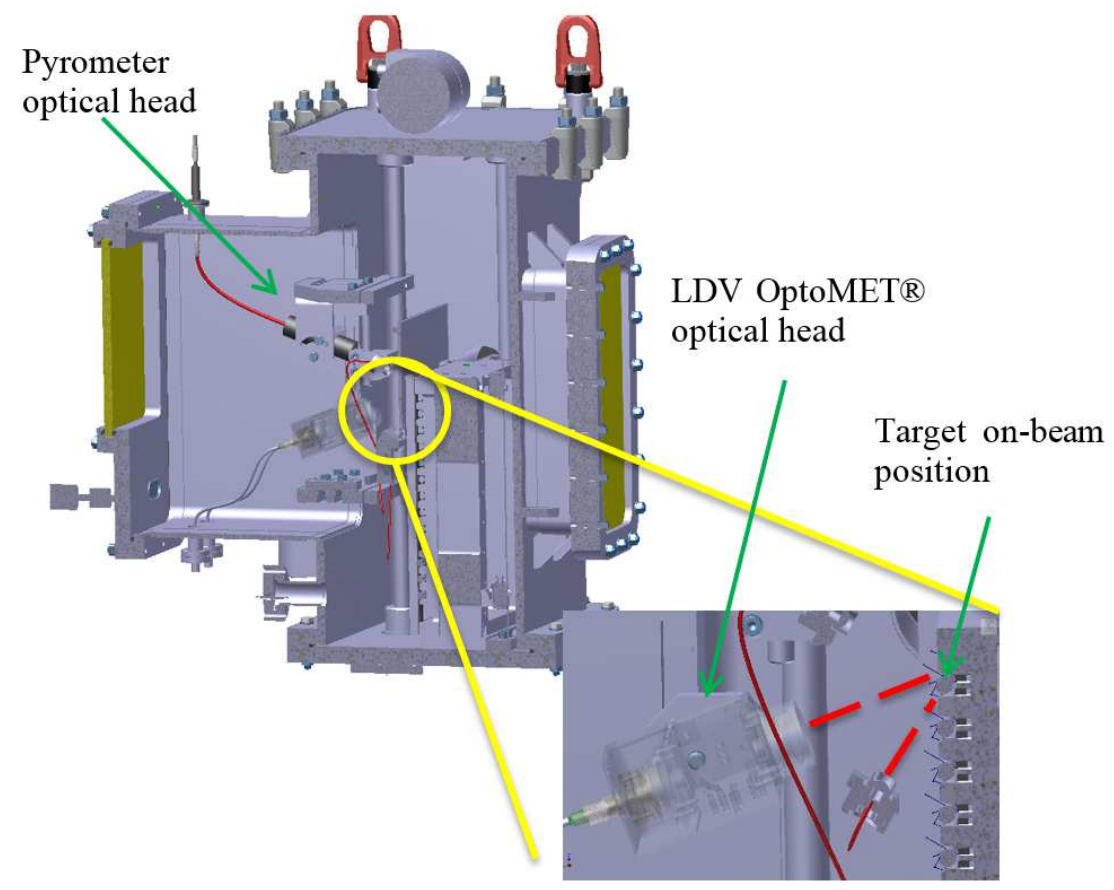

Figure 4.18: Detailed view of the optical instrumentation placed inside the tank, pointing at the surface of the target on-beam position. 
Table 4.1 summarizes the selected instrumentation. As can be seen in the table, all the instrumentation, except the thermocouples, were not in contact with the target surfaces. These thermocouples, which had a much lower acquisition rate, were mainly used for monitoring the temperatures during the experiment since they were not able to record the adiabatic increase of temperature on the target surface. In any case, their recording was also very useful for checking the total deposited energy in the target since they recorded the increase of temperature on the surface after the heat diffusion from inside them. Two thermocouples per target were attached by inserting them in small $1 \mathrm{~mm}$ diameter, $1 \mathrm{~mm}$ depth holes machined in the targets surface. In addition, a ceramic glue which could withstand temperatures above $1000{ }^{\circ} \mathrm{C}$ was added to ensure good thermal contact (figure 4.20-(a)).

No active electronics were placed close to the tank. The velocity and displacement of the target surfaces were measured by passive optical heads pointing at the target on beam position, as shown in figures 4.18 and 4.19. The recorded signals traveled through optic fibres to the instrument control and conditioning electronics and eventually to the DAQ, which were remotely placed in a shielded bunker in the an adjacent tunnel to the facility. The same layout was applied to the pyrometer.

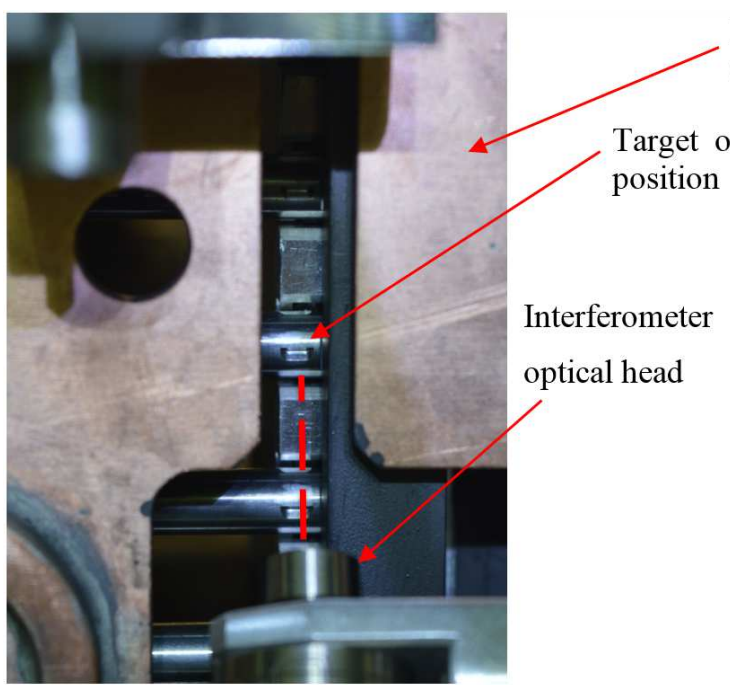

\section{(a)}

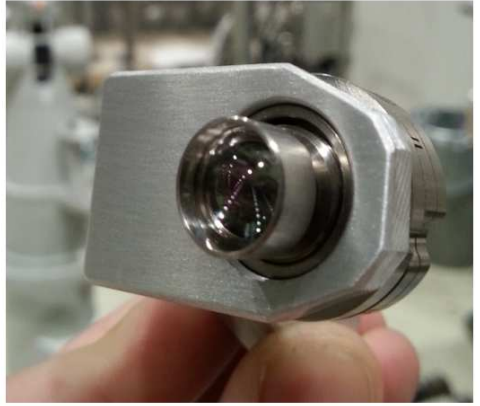

(b)

Figure 4.19: (a) View of the target on-beam position from behind the interferometer head. In the picture it is possible to see the back part of the water cooled copper plate which protected the instrumentation from possible explosions. (b) Interferometer optical head. 
Outside the tank, a radiation hard camera, which can withstand up to $30 \mathrm{kGy}$, monitored the experiment through the radiation resistant glass of the tank as can be seen in figure 4.20-(b). A second conventional HD camera was added in order to get higher quality images during the experiment, although it was assumed that it would be damaged due to the high doses of prompt radiation received, or to single-event-upset induced by high energy hadron fluence. A second radiation hard camera was used in the experiment to point at a Gafchromic foil placed upstream the beam position monitor [209]. This Gafchromic foil darkens as a function of the total dose received by the radiation field, and in this way it can be used as a redundant beam detection system (although it measures only the cumulated "footprint" of the beam).

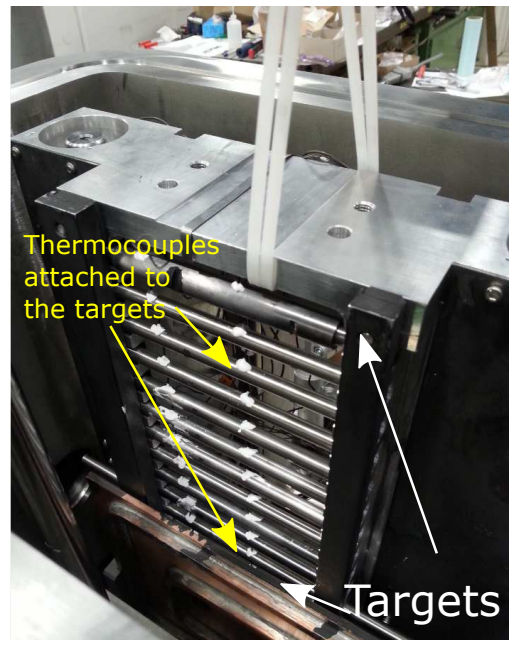

(a)

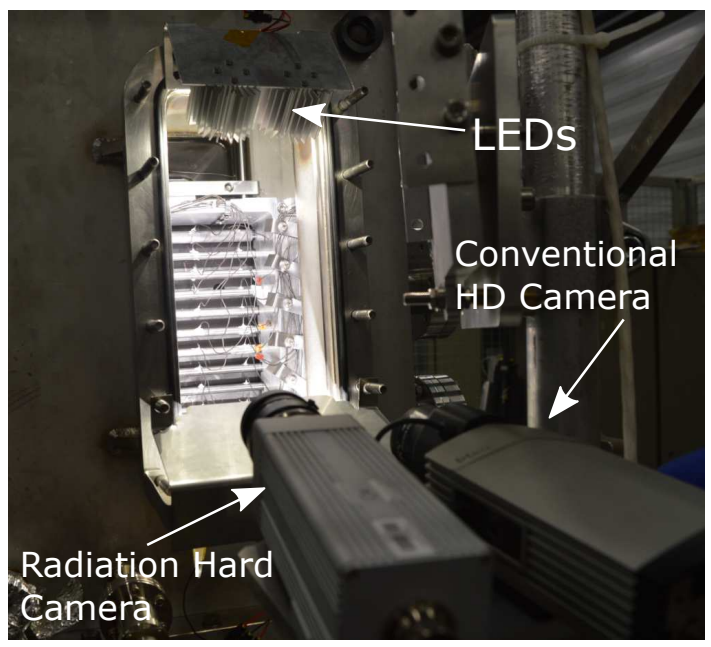

(b)

Figure 4.20: (a) Picture of the installation of the sampleholder inside the vacuum tank showing the thermocouple attached to the targets surface (white glue). (b) Picture of external view of the vacuum tank showing the visual window, the two cameras placed outside the tank, and the targets in the sample holder illuminated by LEDs. 
Table 4.1: Summary of all the instrumentation used in the experiment.

\begin{tabular}{|c|c|c|c|c|c|c|}
\hline Instrument & Number & Measuring & $\begin{array}{l}\text { Maximum } \\
\text { Resolution }\end{array}$ & $\begin{array}{c}\text { Maximum } \\
\text { Range of } \\
\text { Measurement }\end{array}$ & $\begin{array}{l}\text { Acquisition } \\
\text { Rate }\end{array}$ & $\begin{array}{l}\text { Location in the } \\
\text { experiment }\end{array}$ \\
\hline $\begin{array}{l}\text { Interferometer heads } \\
\text { Attocube FPS3010 }\end{array}$ & 2 heads & Displacement & $80 \mathrm{~nm}$ & $2 \mathrm{~m} / \mathrm{s}$ & $10 \mathrm{MHz}$ & $\begin{array}{l}\text { Inside the tank, } \\
\text { pointing at the rod } \\
\text { surface }\end{array}$ \\
\hline LDV OptoMETß & 1 & $\begin{array}{l}\text { Velocity and } \\
\text { Displacement }\end{array}$ & $0.75 \mathrm{~mm} / \mathrm{s}$ & $24.5 \mathrm{~m} / \mathrm{s}$ & $10 \mathrm{MHz}$ & $\begin{array}{l}\text { Inside the tank, } \\
\text { pointing at the rod } \\
\text { surface }\end{array}$ \\
\hline $\begin{array}{l}\text { Pyrometer } \\
\text { IGA 740-LO }\end{array}$ & 1 & Temperature & $0.02{ }^{\circ} \mathrm{C}$ & $300-1400{ }^{\circ} \mathrm{C}$ & $4 \mathrm{MHz}$ & $\begin{array}{l}\text { Inside the tank, } \\
\text { pointing at the rod } \\
\text { surface }\end{array}$ \\
\hline $\begin{array}{l}\text { Thermocouples } \\
\text { (Type K) }\end{array}$ & $\begin{array}{l}26(2 \text { per } \\
\text { target })\end{array}$ & Temperature & $10^{\circ} \mathrm{C}$ & $1500^{\circ} \mathrm{C}$ & $80 \mathrm{~Hz}$ & $\begin{array}{l}\text { Attached to the } \\
\text { targets }\end{array}$ \\
\hline LDV Polytec® & 1 & $\begin{array}{l}\text { Velocity and } \\
\text { Displacement }\end{array}$ & $0.73 \mathrm{~mm} / \mathrm{s}$ & $24 \mathrm{~m} / \mathrm{s}$ & $10 \mathrm{MHz}$ & $\begin{array}{l}\text { In the TT61 } \\
\text { bunker. } \\
\text { tunnel) }\end{array}$ \\
\hline $\begin{array}{l}\text { Radiation Hard } \\
\text { Camera [210] }\end{array}$ & 2 & Video & 400 TVL & - & $25 \mathrm{fps}$ & Outside the tank \\
\hline HD camera [211] & 1 & Video & $1080 \mathrm{p}$ & - & $25 \mathrm{fps}$ & Outside the tank \\
\hline $\begin{array}{l}\text { Pirani } \\
\text { Pressure Gauge }\end{array}$ & 1 & Pressure & - & $10^{-3}$ mbar & $80 \mathrm{~Hz}$ & Inside the tank \\
\hline
\end{tabular}




\subsubsection{Thermal and Structural Calculations of the Experimental Setup}

During the experiment, a considerable amount of heat had to be deposited in the high density targets. This heat had to be diffused and released to the ambient without any unexpected overheating of the targets supporting structures/in-tank instrumentation or sample holder guiding shafts, situation which may lead to loss of precision and alignment. In addition, the stresses of the tank walls and their deformations due to vacuum had to be checked as well. In order assess theses concerns, a complete set of thermochemical analysis of different parts of the experimental setup was carried out during the design phase. These analysis initially included transient Computational Fluid Dynamic (CFD) calculations during the first stages of the design, in which it was considered that the tank could have been filled by pressurized helium instead of being in vacuum in order to improve the heat transfer and cooling of the irradiated targets. Finally, this option was abandoned since CFD calculations showed that the helium could overheat very fast (reaching temperatures above $150{ }^{\circ} \mathrm{C}$ as shown in figure 4.21 ) which would endanger the in-tank instrumentation heads.

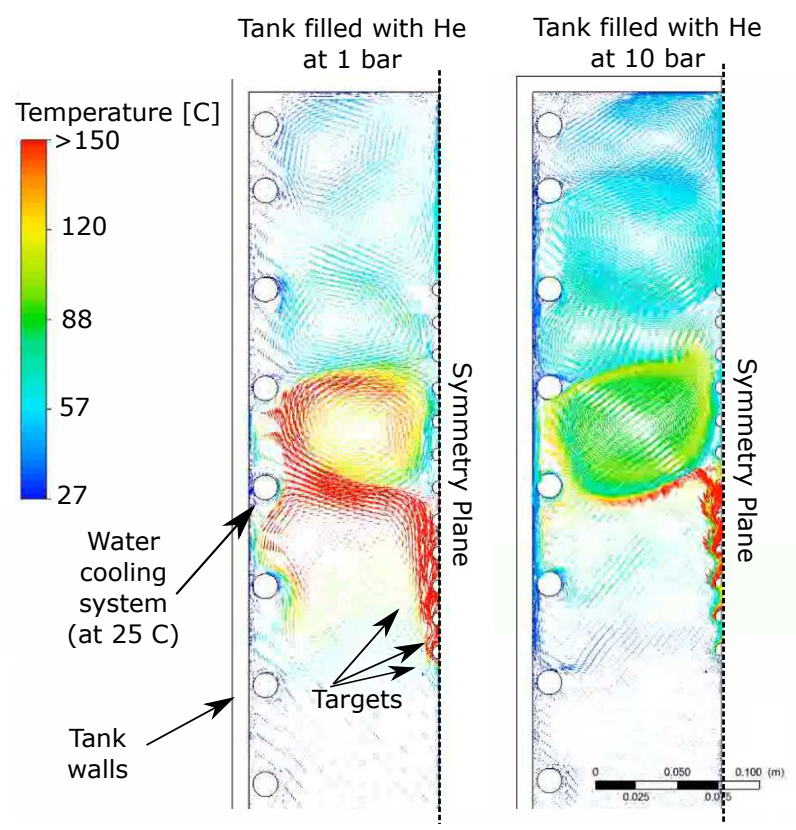

Figure 4.21: Snapshots of He streamlines from natural convection obtained by transient 2D-CFD simulations on a preliminary simplified geometry of the HRMT27 tank during first design phase. Concept of tank filled by He was abandoned since streamlines reached temperatures were well above $150{ }^{\circ} \mathrm{C}$ (in-tank instrumentation heads temperature limitation). 
After the consideration that a vacuum tank would be the most suitable solution, three kind of FE ANSYS Mechanical@ calculations were performed:

- Transient thermal analysis of heat deposited in the targets and subsequent heat transfer towards the tank walls and ambient during the whole duration of the experiment.

- Static structural analysis of thermal expansions of the most critical structures such as sample holder, sample holder guiding shafts, and instrumentation shafts at the most unfavorable moment (when the highest temperatures are reached).

- Structural calculations of deformation and stresses of the tank walls due to internal vacuum.

For the full heat transfer transient analysis, it was necessary to simulate the initially foreseen $\sim 144$ proton pulse bursts in the 13 targets, as well as to include in the computational model a realistic geometry of instrumentation heads, supports, instrumentation protection screen and guiding shafts. All the tank walls and internal structures need to be taken into account in the model in order to simulate precisely the radiation heat transfer from the targets to the ambient. Figure 4.22 shows different views of the parts included in FE model, from the tank walls, to all the internal structures. Furthermore, detailed geometry of the target support system including graphite $\mathrm{V}$-shape holders and strings was taken into account since they were one of the most critical parts for being in direct contact with the irradiated targets.

The deposited energy on the targets over the time was consistently simulated taking into account the real irradiation sequence of the experiment. This sequence consisted of several impacted pulses per target at each intensity, which was progressively increased from $10^{11} \mathrm{ppp}$ to $1.5 \cdot 10^{12} \mathrm{ppp}$ in several steps. It was conservatively assumed that during the experiment the 13 targets will receive beam at a given intensity spaced by $30 \mathrm{~s}$ (one pulse per target), followed by a pause of $12 \mathrm{~min}$ before the next corresponding irradiation series at the same intensity or above. This would lead to a period of approximately 18 min between two consecutive pulses seen by an individual target ${ }^{1}$. This irradiation sequence is explained more in detail in section 4.6.2. The tank was maintained under vacuum, therefore most of the heat was transferred from the targets to the tank walls by radiation heat transfer. In order to take that into account, the option "surface to surface" radiation heat transfer was used in the FE simulations. Total energy deposited

\footnotetext{
${ }^{1}$ In reality, during the experiment the repetition rate of impacted pulses on targets was much slower than the initially foreseen due to coordination with SPS operation and necessary instrumentation crosschecks after each pulse. The time between two consecutive pulses was rarely below 5 mins and in most of the cases, each target was seeing a pulse each $1 \mathrm{~h}$.
} 
inside the targets was obtained by FLUKA Monte Carlo simulations and applied as averaged in volume internal heat source in the ANSYSR model.
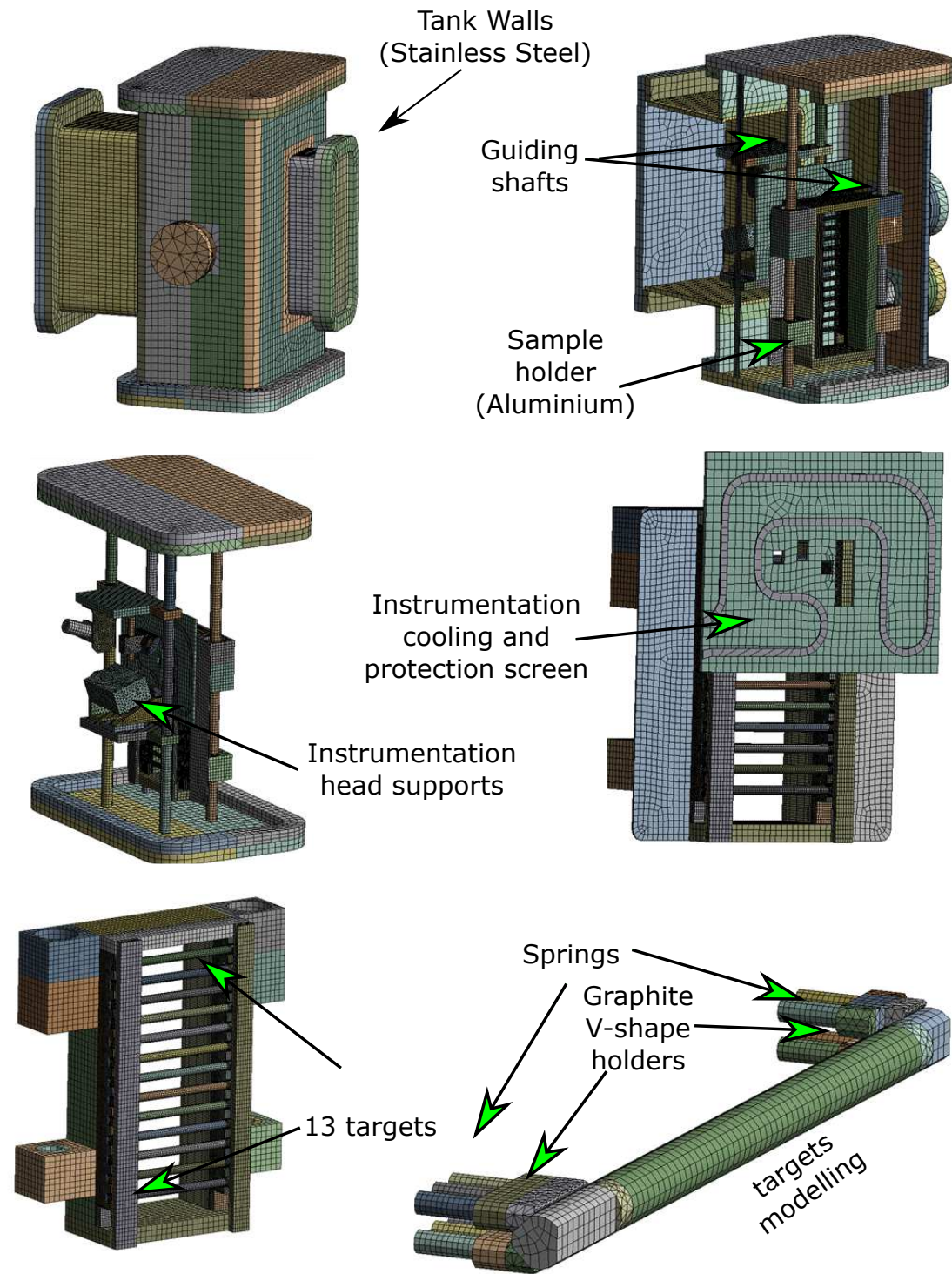

Figure 4.22: Screenshots of different parts of the modeled geometry of the experimental setup. 


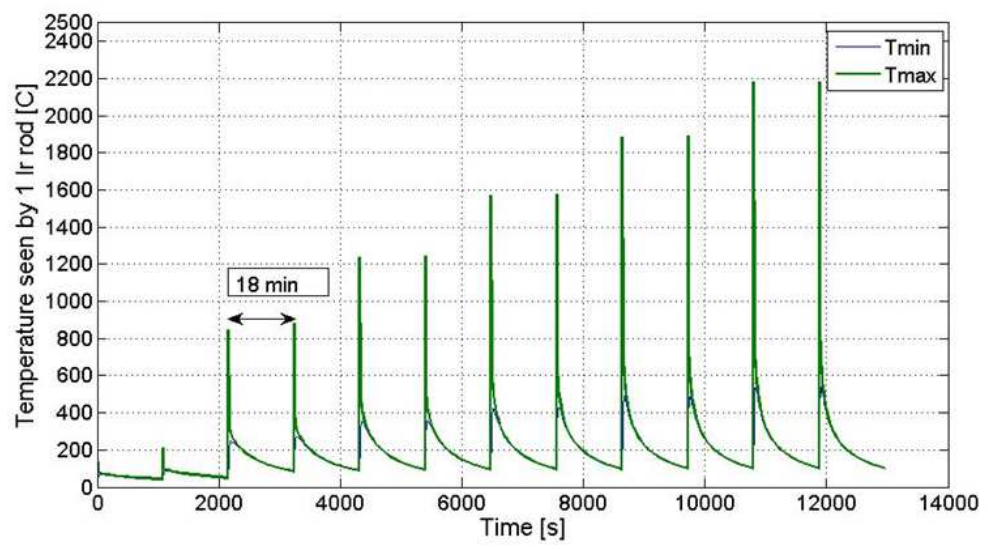

Figure 4.23: Estimated temperature reached in an iridium target during the whole experiment according to the initially foreseen irradiation sequence

Figure 4.23 presents the maximum temperature seen by the most unfavorable target (iridium) receiving an increasing intensity beam each 18 minutes, as described in the irradiation configuration assumed. This plot shows that with the selected sequence, the iridium will safely stay below its melting point of $2450{ }^{\circ} \mathrm{C}$ and that before each impact the temperature of the rod would be in the order of $100{ }^{\circ} \mathrm{C}$. In any case, online monitoring of rods temperature was carried out in order to prevent any unexpected overheating.

Figure 4.24-(a)-(b) shows the temperature history reached in the graphite clamps over the 180 min duration considered for the experiment. As can be seen in the plot, heat is efficiently transfer by conduction to the rest of sample holder body, preventing graphite overheating due to accumulation of heat coming from the targets. Estimated maximum temperature of $640{ }^{\circ} \mathrm{C}$ is reached at high intensity pulses. This temperature is well below the melting point of graphite and no oxidation of the clamps is expected thanks to vacuum.

In a similar way, figure 4.24-(a)-(b) shows maximum temperatures reached in the aluminum sample holder, which was considered one of the most critical points given that at high intensity the surface of the targets could reach up to $740{ }^{\circ} \mathrm{C}$, well above the melting point of aluminum of $660{ }^{\circ} \mathrm{C}$ and, even if targets-aluminum are not in direct contact, they are very close. Nevertheless, it was shown that the maximum temperature reached would be $180{ }^{\circ} \mathrm{C}$, well below the melting point. This is thanks to the high thermal conductivity of aluminum, which rapidly distribute the incoming heat from the targets within the whole sample holder volume. 


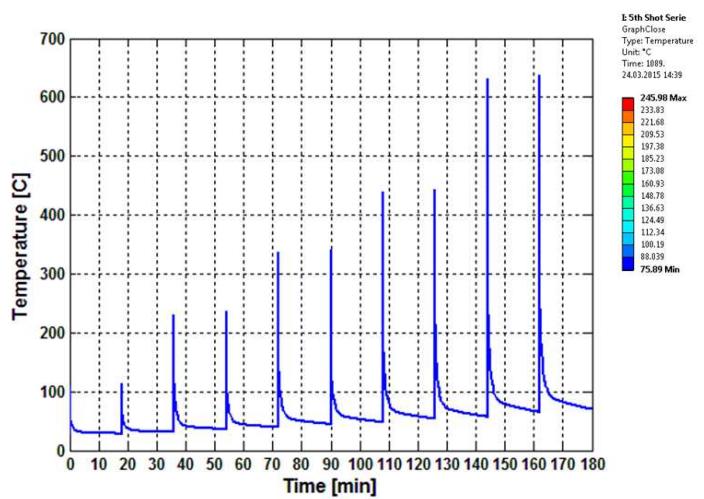

(a)

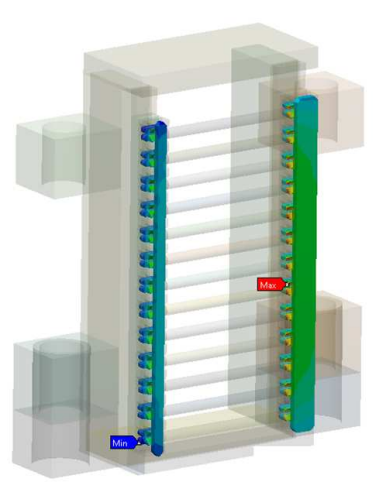

(b)

Figure 4.24: (a) Estimated maximum temperature history in the graphite supports during of the experiment. (b) Estimated temperature distribution in the graphite supports in direct contact with the targets.

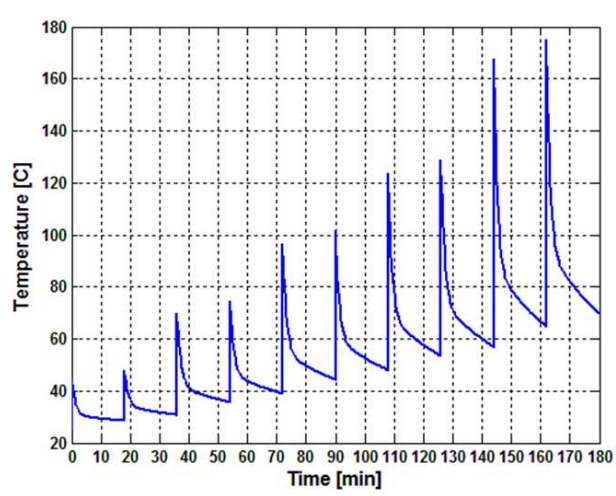

(a)

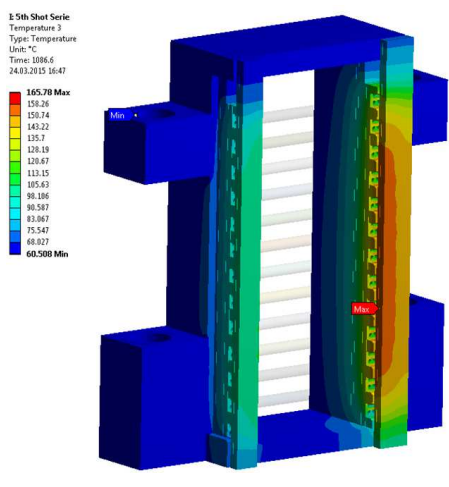

(b)

Figure 4.25: (a) Estimated maximum temperature history in the aluminum sample holder during of the experiment. (b) Estimated temperature distribution in the in the aluminum sample holder.

Figure 4.26 shows the temperature profiles reached on the tank walls, bases, and instrumentation heads at the corresponding most unfavorable times. As can be seen in the figures, overheating of the tank walls beyond $40{ }^{\circ} \mathrm{C}$ and tank bases beyond $45{ }^{\circ} \mathrm{C}$ was not expected. Furthermore, the instrumentation was well protected from radiative heat transfer from the targets by the cooled instrumentation screen shown in figure 4.19-(a). Maximum temperature of $30{ }^{\circ} \mathrm{C}$ was expected there, well below the instrumentation limiting working temperature of $60^{\circ} \mathrm{C}$. 


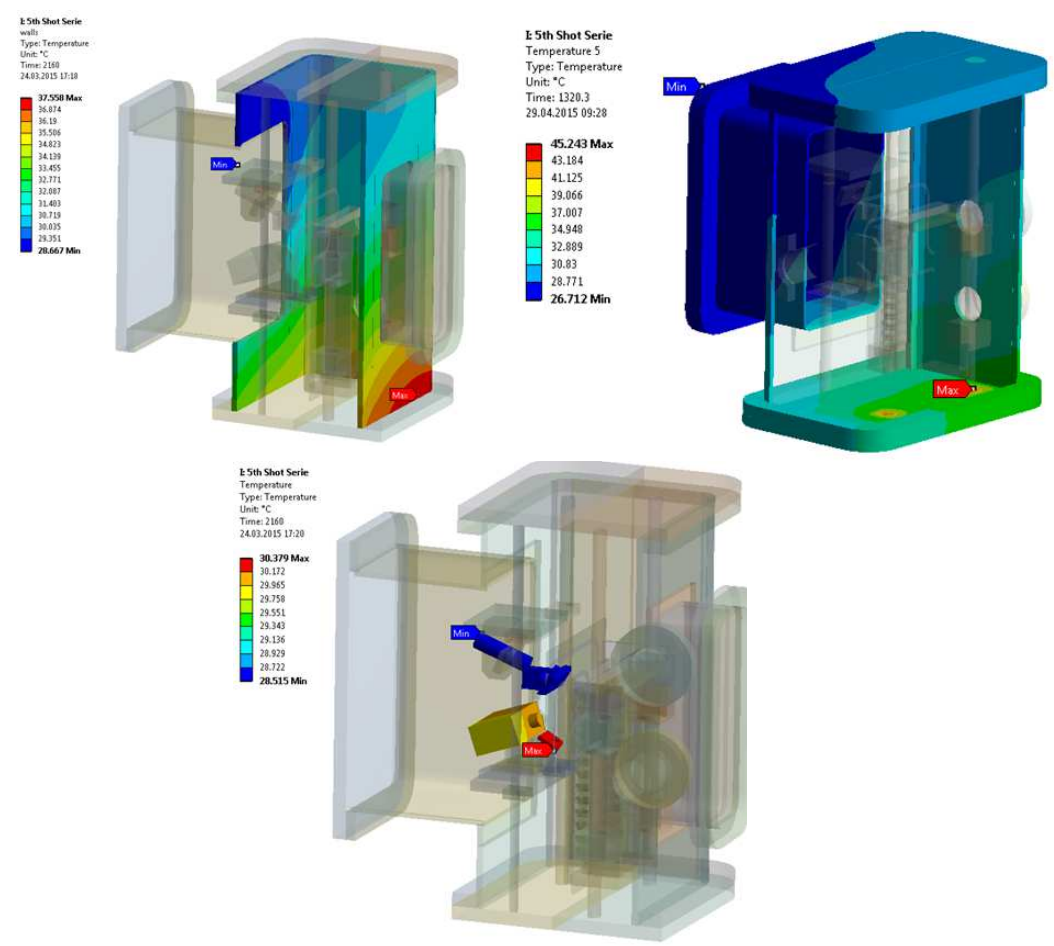

Figure 4.26: Contour plot of temperature profiles in the tank walls, bases and instrumentation heads at the most unfavorable time.

A thermal expansion associated to the recently presented temperature increase due to heat diffusion from the targets to the aluminum sample holder would certainly take place. Therefore, structural simulations were carried out to assess two critical aspects that this thermal expansion could cause; (i) vertical expansion of the sample holder which could make that the farthest target from the attachment point of the moving system losses precision in vertical alignment with the incoming proton beam. (ii) Horizontal expansion of the sample holder which could change the distance between its guiding shafts, causing a catastrophic stuck of the sample holder during the on-beam/target shifting phase.

The most unfavorable time for the two named phenomena is presented in figure 4.27-(a), which corresponds to the time in which a considerable amount of heat from all the irradiation history has diffused from the targets to the sample holder and homogeneously distributed along its body. Figure 4.27-(a) shows that the whole volume of the sample holder at this time is between $60{ }^{\circ} \mathrm{C}$ and $64{ }^{\circ} \mathrm{C}$. Figures 4.27-(b)-(c) show the two deformations of concern. Thermal expansion of the sample holder would produce a maximum vertical displacement of $0.3 \mathrm{~mm}$ at 
the farthest target from the attached part of the target holder. This misalignment, even if considerably large in comparison with the set alignment precision - $0.1 \mathrm{~mm}-$, was considered acceptable for the instrumentation alignments. It is also important to note that this effect would have taken place only at the farthest target and at the last high intensity pulse. In addition, finally the time between consecutive pulses and cooling times were much longer than the ones initially foreseen, as therefore is highly unprovable that this $0.3 \mathrm{~mm}$ thermal-induced misalignment took place. In a similar manner, figures 4.27-(c) shows that the potential maximum deformation at the guiding shafts would be $0.26 \mathrm{~mm}$. Part of this deformation could be absorbed by the bearing of the movable contact. In addition, the top part of one of the guiding shaft was deliberately made unconstrained. In this way the shaft could bend easily, accommodating this deformation and avoiding that sample holder would have got stuck.

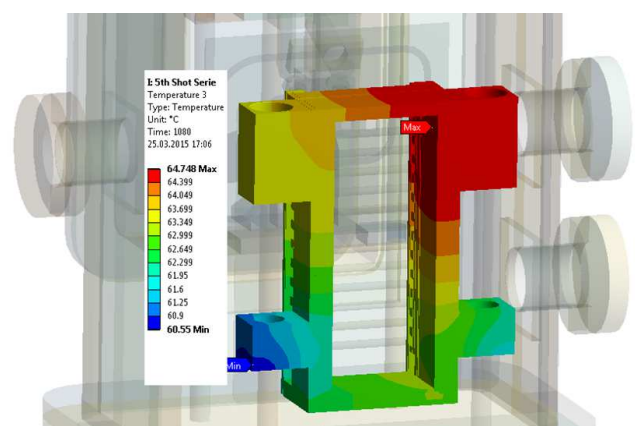

(a) Temperature of Sample Holder before high
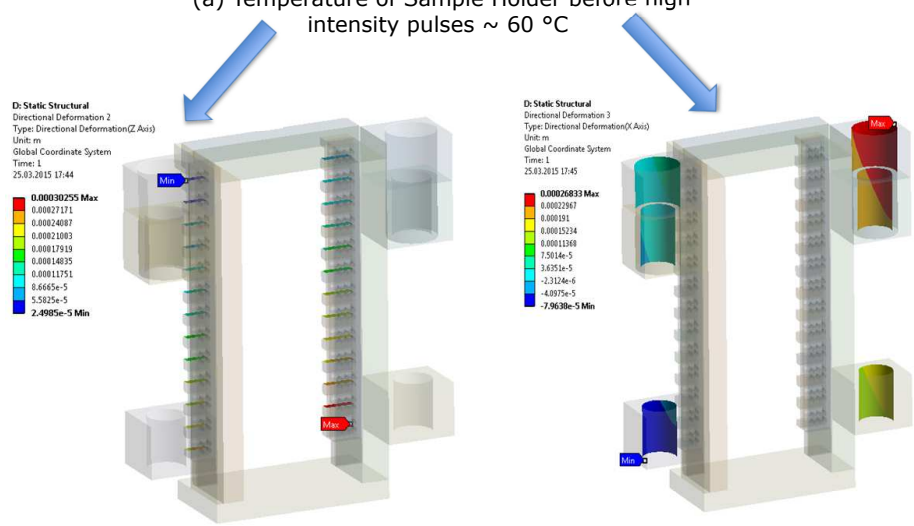

(b) Vertical expansion of sample holder.

Max $\vee$ expansion at target position $=0.3 \mathrm{~mm}$

(c) Horizontal displacement at the guiding bores. Max offset at guiding shaft: $0.26 \mathrm{~mm}$

Figure 4.27: Contour plots of the thermo-mechanical calculation performed of the sample holder. (a) Thermal load imposed into the model. (c) Vertical expansion (b) Displacement of the guiding shaft. 
Finally, a structural analysis of the deformation of the tank walls due to internal vacuum is included. This simulation had two main purposes; (i) resolve the stress state of the tank walls and identify any critical point where its structural stability could be compromised. (ii) Resolve the deformation of the tank and make sure this deformation would not affect the instrumentation and targets alignment.

Figure 4.28 shows a x200 oversized deformation plot with the von Mises stresses reached at the tank walls due to internal vacuum. As can be seen in the figure, triangular stiffeners were added in the corners of connection between the lateral bodies and the main tank body. These corners were acting as stress concentrators while with the addition of the stiffeners the reached stresses were $76 \mathrm{MPa}$, well below the yield strength of the stainless steel 304L used for the tank. It was checked as well that the vertical displacements of the areas where the sample holder and instrumentation guiding shafts were negligible.

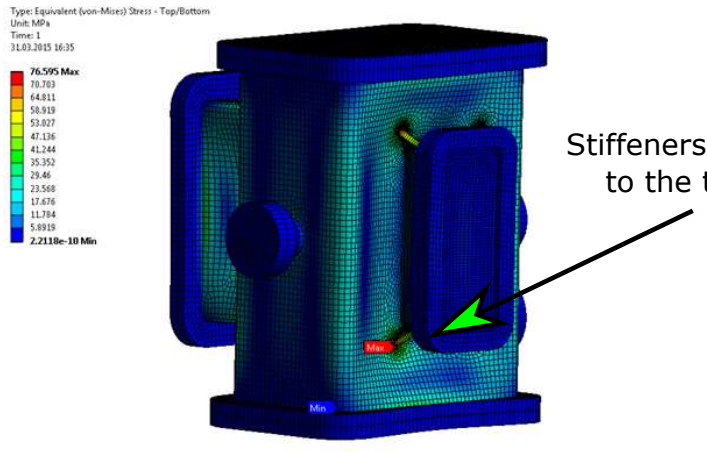

(a)

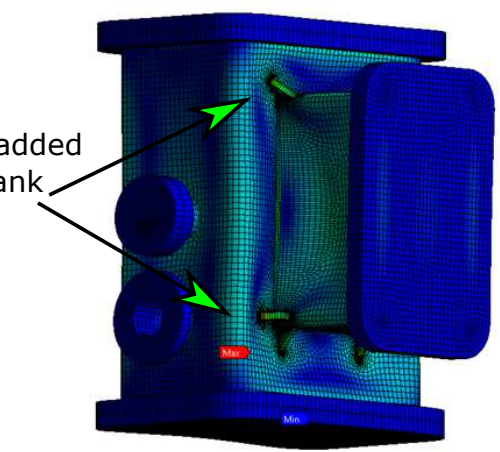

(b)

Figure 4.28: Contour plots of the structural simulations performed on the stainless steel vacuum tank subjected to the external atmospheric pressure. The plots show augmented deformation and von Mises stresses. Addition of triangular stiffeners in the corners of connection between the lateral bodies and the main tank body reduced significantly the reach stresses. 


\subsection{List of Investigated Target Materials}

Table 4.2 shows the list of the 13 targets irradiated in the experiment together with a picture of the sample holder of the picture, which shows the respective targets. The order of the targets in the table is consistent with the position of each of the targets in the sample holder. The selection of these target materials was done based on possible candidates for the future AD-Target, as described in the literature review of section 1.5 in Chapter 1. More detailed information regarding the supplier, manufacturing process and micro-structure of the different targets is provided in the next subsections 4.5.1-4.5.5.

Table 4.2: List of targets irradiated during the HRMT27 experiment.

\begin{tabular}{|c|c|c|c|c|c|}
\hline $\begin{array}{l}\text { Target } \\
\text { No }\end{array}$ & $\begin{array}{c}\text { Target } \\
\text { Identifier }\end{array}$ & Material & $\begin{array}{l}\text { Density } \\
{\left[\mathrm{g} / \mathrm{cm}^{3}\right]}\end{array}$ & $\begin{array}{l}\text { Target } \\
\text { Length } \\
{[\mathrm{mm}]}\end{array}$ & $\begin{array}{l}\text { Picture of the } 13 \text { targets } \\
\text { placed in the sample holder. } \\
\text { Same order of targets as rows } \\
\text { in the table }\end{array}$ \\
\hline \#13 & W-Ta & $\begin{array}{l}\text { Pure W } \\
\text { Cladded in } \\
2 \mathrm{~mm} \mathrm{Ta}\end{array}$ & - & 140 & \\
\hline \#12 & Ir3 & Pure Ir & 22.4 & 140 & \\
\hline \#11 & Ir2 & Pure Ir & 22.4 & 140 & \\
\hline$\# 10$ & Ir 1 & Pure Ir & 22.4 & 140 & \\
\hline$\# 9$ & W-La & $\mathrm{W}+\mathrm{La}_{2} \mathrm{O}_{3}$ & 19.3 & 140 & \\
\hline$\# 8$ & W3 & Pure W & 19.3 & 140 & \\
\hline$\# 7$ & W2 & Pure W & 19.3 & 140 & \\
\hline$\# 6$ & W1 & Pure W & 19.3 & 140 & \\
\hline$\# 5$ & Ta2 & Pure Ta & 16.6 & 170 & 0 \\
\hline$\# 4$ & Ta1 & Pure Ta & 16.6 & 170 & \\
\hline$\# 3$ & TZM2 & $\begin{array}{l}\text { Mo-Ti-Zr } \\
\text { Alloy }\end{array}$ & 10.2 & 240 & \\
\hline$\# 2$ & TZM1 & $\begin{array}{l}\text { Mo-Ti-Zr } \\
\text { Alloy }\end{array}$ & 10.2 & 240 & $\bar{a}$ \\
\hline$\# 1$ & Mo & Pure Mo & 10.2 & 240 & \\
\hline
\end{tabular}




\subsubsection{Iridium}

As introduced in the literature review of Chapter 1 , the inclusion of this material in the HRMT27 experiment is more than well justified by the fact that this is the current target core material, selected by its high density in the last AD-Target design at the late 80's. Figure 4.29 shows a picture of one of the HRMT27 iridium targets before irradiation. The raw material for this target was provided by Goodfellow [212]. The rod was manufactured from a drip melted ingot by electron beam melting. The drip melt ingot was then forged and swaged into a rod of $8 \mathrm{~mm}$ diameter. Figure 4.30 shows SEM analysis of the micro-structure of a similar Ir material (same supplier and manufacturing process) as the one used for the target in the experiment. In the figure it can be observed that the micro-structure is heterogeneous (probably due to annealing during the manufacturing process) and the grain size is in the order of a few microns.

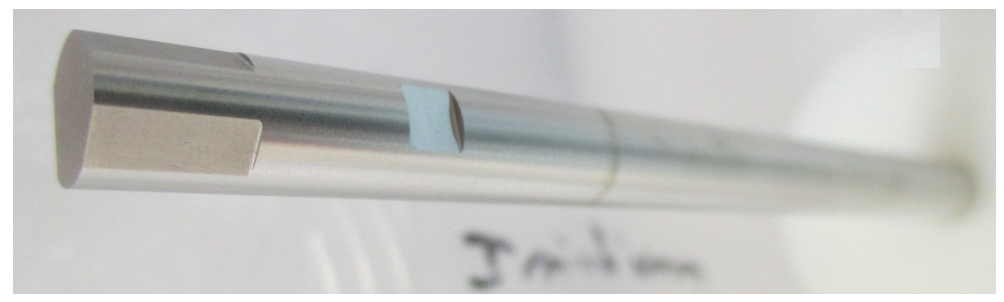

Figure 4.29: Picture of a Ir target prior irradiation in the HRMT27 experiment.

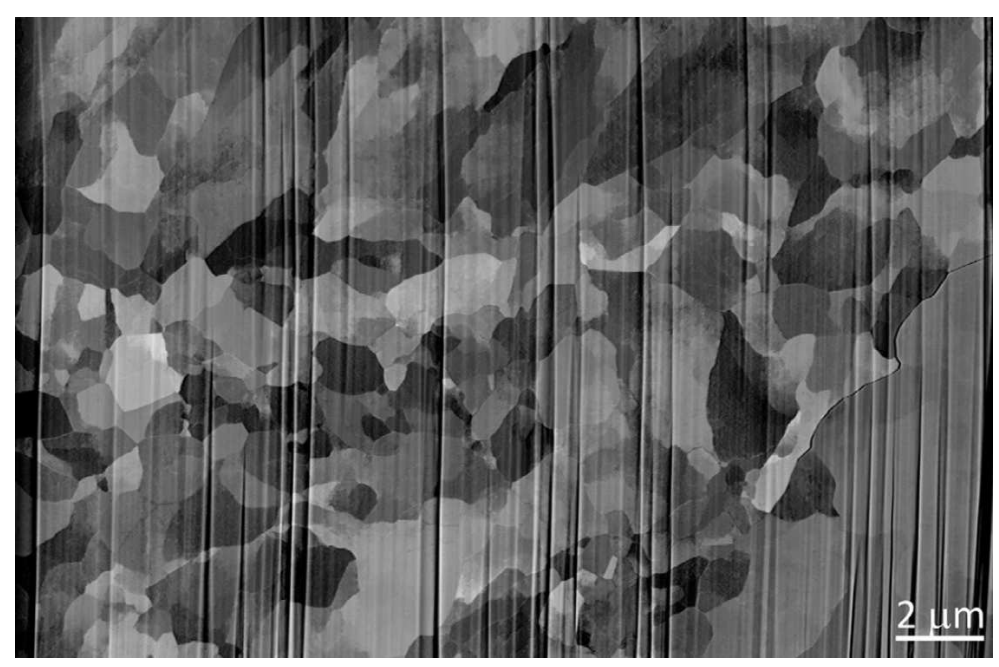

Figure 4.30: SEM observation of the micro-structure of a similar Ir material as the one used in the target. Courtesy of A. Perez Fontenla. 


\subsubsection{Pure Tungsten and Tungsten doped with Lanthanum Oxide}

As introduced in Chapter 1, the inclusion of pure Tungsten in the HRMT27 experiment was supported by, in addition to its very suitable density, the experience and interest on this material from in the scientific community, and in particular for its application to neutron spallation targets. For example, pure tungsten has been used as core material for the ISIS spallation source at RAL (Rutherford Appleton Laboratory) [90] and it is a possible candidate for the future design of CERN's n_TOF spallation target [91] and the target of the Neutrino Factory [92]. Furthermore, pure tungsten is the baseline candidate material for other future high powered targets such as the one of the European Spallation Source (ESS) in Lund [93] and the potential BDF (Beam Dump Facility) at CERN [94]. These applications also support the inclusion of pure tungsten in the experiment, since the information of its behavior under proton beam impacts could be transversely applied to them.

A picture of one of the pure $\mathrm{W}$ targets irradiated during the experiment was shown in figure 4.3. The raw material for this target was provided by PLANSEE. The rods were produced starting from metal powder, pressed, sintered and hot forged with stress relieve heat treatment after hot working. Maximum grain size guaranteed for this material is $40 \mu \mathrm{m}$. Figure 4.31 shows a microscope image of the cross section of a $8 \mathrm{~mm}$ rod similar to the irradiated targets. High uniformity and fine grains can be observed.

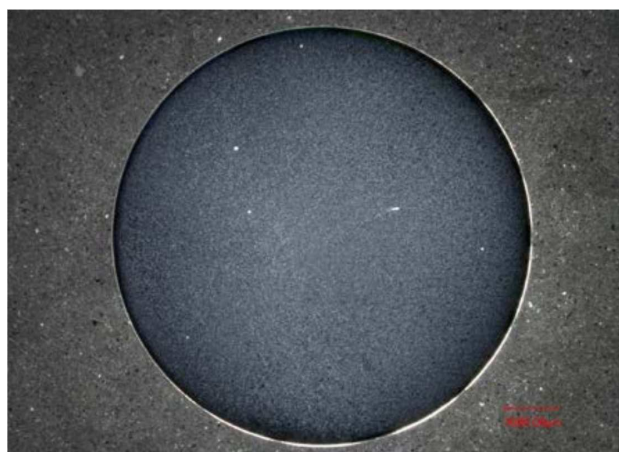

Figure 4.31: Microscope images of a cross section of a $8 \mathrm{~mm}$ tungsten rod similar to the irradiated targets. Courtesy of A. Perez Fontenla [213].

Furthermore, an additional target made of Tungsten doped with Lanthanum Oxide $\left(\mathrm{W}-\mathrm{La}_{2} \mathrm{O}_{3}\right)$ was added to the experiment to study the response of its improved mechanical properties at high temperature in comparison to the ones of pure $\mathrm{W}$ as described in section 1.5.3 of Chapter 1 . Similarly as the pure W targets, the raw 
material for the $\mathrm{W}-\mathrm{La}_{2} \mathrm{O}_{3}$ targets was provided by Plansee in form of rods, which were produced starting from metal powder, pressed, sintered and hot forged with stress relieve heat treatment after hot working.

\subsubsection{Tantalum}

As introduced in section 1.5.3 of Chapter 1, tantalum was added to the studies of this thesis and the present experiment due to its well known ductility in comparison to the other tested materials. Furthermore, tantalum, as already mentioned, presents very high resistance to spall. Given that, due to the large level of stresses taking place in the experiment, it is assumed that all of the targets would experience plastic deformation in any case. It was considered interesting to introduce tantalum in the experiment in order to compare possible failure mechanisms between materials with known different properties and ductility.

Figure 4.32 shows a picture of one of the Ta targets prior to irradiation in the HRMT27 experiment. The raw Ta material was provided by PLANSEE, corresponding to its designated "Ta melting quality". The rods were produced out of melted pre-material ingots, ground, and delivered in annealed condition. These rods had therefore a particularly low yield strength $(\geq 105 \mathrm{MPa})$ and very high elongation to break $(\geq 25 \%)[214]$.

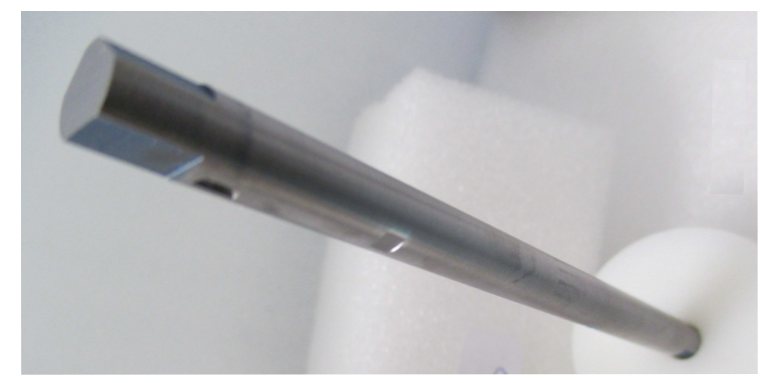

Figure 4.32: Picture of a Ta target prior irradiation in the HRMT27 experiment. Note that these targets (\#5Ta,\#6Ta) were slightly longer $(160 \mathrm{~mm}$ instead of $140 \mathrm{~mm}$ ) to compensate its longer interaction length due to its lower density in comparison to $\mathrm{W}$ and Ir. 


\subsubsection{Molybdenum and TZM}

It was considered interesting to add Mo and TZM to the HRMT27 experiment in order to assess the fact that a lower density material could have more chance to survive the conditions reached in the AD-Target core. In addition, molybdenum and its alloys (such as TZM) are of interest in future R\&D projects on targets at CERN, since it will be one of the target materials of the potential Beam Dump Facility [94].

A picture of the TZM target before irradiation is shown in figure 4.33. Raw Mo and TZM materials were provided by PLANSEE in form of rods of $8 \mathrm{~mm}$ diameter. The rods were produced starting by metal powered, pressed, sintered and hot forged with stress relieve heat treatment after hot working.

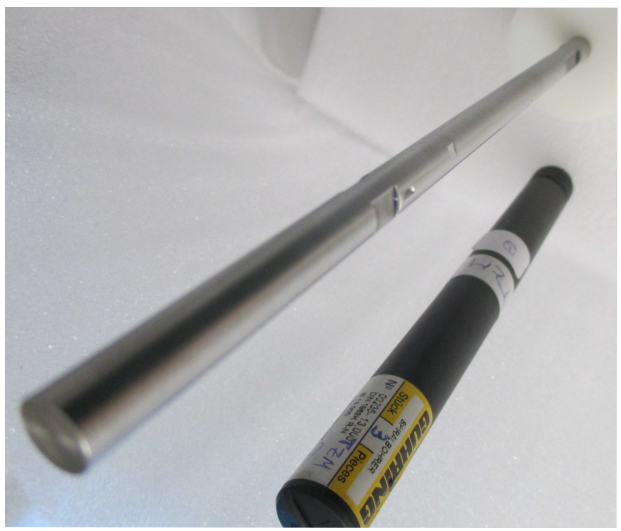

Figure 4.33: Picture of a TZM target prior to irradiation in the HRMT27 experiment. Note that these targets (\#1Mo,\#2TZM1 and \#3TZM2) had a length of $240 \mathrm{~mm}$ instead of $140 \mathrm{~mm}$ to compensate its longer interaction length due to its lower density

\subsubsection{Tungsten Cladded in Tantalum}

Finally, the last material that was irradiated was a tungsten target cladded in tantalum. The motivation of the inclusion of this target comes from the study presented in section 3.5 of Chapter 3 of the present thesis, in which it was numerically shown that the addition of a high density cladding could effectively reduce the level of tensile pressures reached in the core of the AD-Target. An experimental test of the efficacy of this strategy was necessary since, in reality, several uncertainties that are not taken into account by the simulations exist, such as the quality of the core-cladding interface and potential detachments due to the plastic deformation induced by the pressure wave. $R \& D$ of manufacturing and behavior of tantalum cladding for high density targets is also in the interest of other high 
powered target projects such as ISIS target at RAL [215, 216] and the potential new targets of $\mathrm{n}_{\text {_ TOF }}$ and the BDF facility at CERN. The interest of this Ta cladding in these facilities does not come from impedance mismatch or dynamic phenomena as in the AD-Target, but from the enhancement of corrosion protection of Ta against the high speed water cooling circuit surrounding the targets core. Nevertheless, lessons learned from manufacturing and response of this cladding in the present study could also benefit these applications.

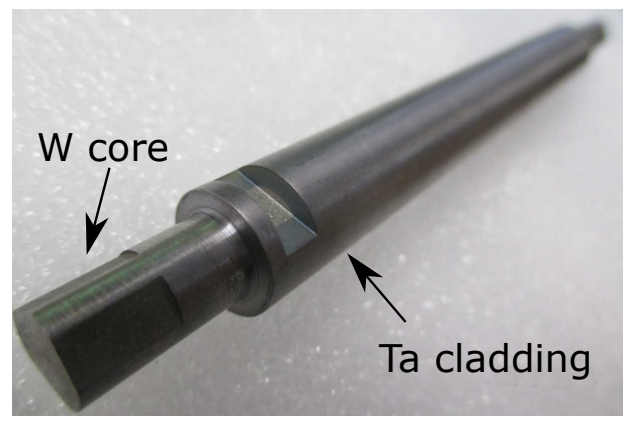

Figure 4.34: Picture of the $\mathrm{W}$ cladded in Tantalum target prior to irradiation in the HRMT27 experiment. Named \#13W-Ta in table 4.2.

Several strategies were initially considered for manufacturing this of Ta-cladded target. First attempt included a coating of Ta by chemical vapor depositions process (CVD) up to a thickness of $480 \mu \mathrm{m}$ [217]. However, this strategy was abandoned since the achieved thickness was not enough, the morphology of the coating was inhomogeneous (limiting its structural stability) as well as SEM observation indicated local detachments of the coating at the interface. A second strategy consisted in a W rod sleeved inside a Ta-canning [218] . However, several detachments were also observed by SEM in this case. Finally, the manufacturing method selected was the same as the currently used in the ISIS spallation target [215], consisting in Ta-cladding in tungsten via Hot Isostatic Pressing (HIPing) process. This process was carried out by Advanced Technology \& Materials Co. (AT\&M). Figure 4.34 shows the cladded target irradiated in the experiment. As can be observed in the picture, the finished target was only partially cladded in order to be compatible with the sample holder. This partial cladding was manufactured by machining out the cladding in the rod extremities. 
a)

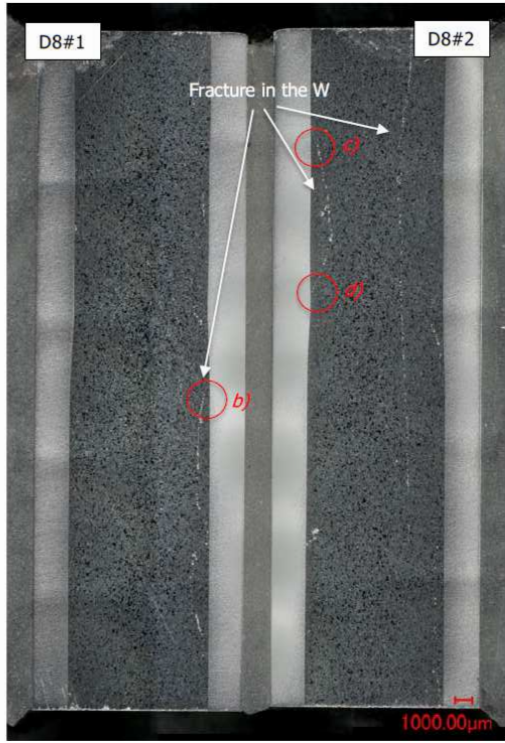

b)

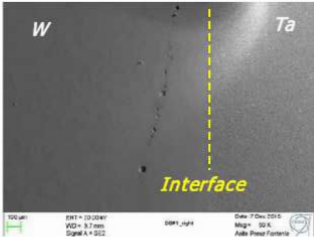

c)

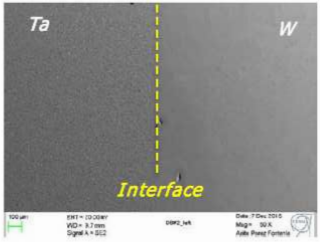

d)

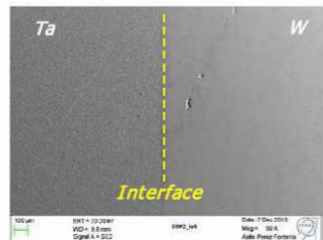

Figure 4.35: (a) Optical microscopy $(30 \mathrm{x})$ after mechanical polishing of a longitudinal cut of a twin W-Ta cladded target. (b-d) SEM images of some regions close to the W-Ta interface. As can be seen in the pictures, very good bonding and a continuous interface exists. Courtesy of A. Perez Fontenla [213]

Figures 4.35 and 4.36 show images of microscope and SEM analysis carried out in twin targets to the irradiated one. In figure 4.34-(b)-(d) it is shown by SEM that the interface and W-Ta bounding is very good. However, internal porosity and cracks shown in figure 4.34-(a) and grain inhomogeneity in 4.36 indicate that quality of this tungsten is not as good as the one of the pure $\mathrm{W}$ target provided by PLANSEE shown in figure 4.31, complicating the repeatability and direct comparisons between the response of non-cladded and cladded targets during the experiment. This grain inhomogeneity and big grain sizes was probably a consequence of recrystallization during the HIP process due to the high temperatures reached. 
a)

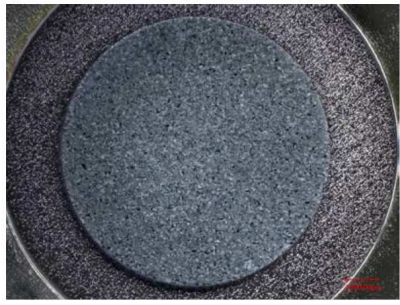

c)

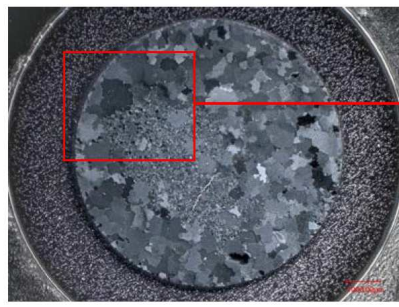

b)

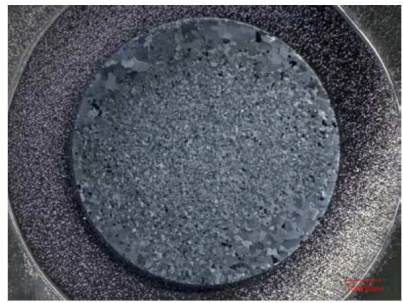

d)

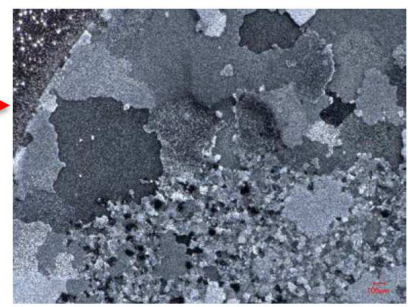

Figure 4.36: Optical microscopy $(30 \mathrm{x})$ in various transversal sections of a twin $\mathrm{W}$ Ta cladded target. d) Detail at 100x. Unfortunately, a substantial amount of porosity and inhomogeneity of the $\mathrm{W}$ material and grain growth can be observed. Therefore, quality of this tungsten is not as good as the one of the pure $\mathrm{W}$ target provided by PLANSEE, complicating the repeatability and direct comparisons between the response of non-cladded and cladded targets. Courtesy of A. Perez Fontenla [213] 


\subsection{Execution of the Experiment}

\subsubsection{Targets Alignment Procedure}

As anticipated in section 4.3.2, the accurate alignment of the $8 \mathrm{~mm}$ diameter experimental targets is one of the most important tasks in order to guarantee the success of the experiment. This alignment had to fulfill the following specifications:

- Each of the longitudinal axis of the target rods had to be aligned with the incoming beam.

- Mirrors of the on-beam position target had to be aligned with the optical instrumentation.

- BPKG/BTV (beam position monitors and beam transverse size monitoring) had to be aligned with tank and the on-beam-position target.

To accomplish these objectives a multistage procedure was conceived, consisting of:

1. Accurate measurement of target positions in the sample holder: In this step a coordinate measuring machine (CMM) was used in order to scan and measure with high precision the relative position of the axis of each of the targets with respect to a laser tracker prism installed on the top of the sample holder.

2. Alignment of the sample holder with the BPKG/BTV: Once the sample holder was installed inside the tank, the BPKG/BTV was aligned with the first target of the sample holder using as well a laser tracker prism installed on top of the BPKG/BTV assembly and the same laser tracker of the sample holder used in the previous step. The relative position of all the targets with respect the laser tracker of the sample holder was known from the CMM measurements of step 1. By taking these references, it was set as well the exact position for the step motor and the LVDT system, which controlled the vertical movement of the sample holder.

3. Instrumentation head alignment: The instrumentation heads pointing at the mirrors of the on-beam position were aligned by finely adjusting the instrumentation supports and tuning the optical lens. Possible minor deviations between the nominal positions of the different targets were compensated within the vertical size of the mirrors, which allow some margin of a few millimeters.

4. BPKG/tank/experimental table pre-alignment with "theoretical beam" position: in the surface building of the HiRadMat facility, the whole tank was pre-aligned with a reference of the "theoretical position of the beam" ad- 
justing all degrees of freedom (horizontal, vertical and angle rotation) of the experimental table. During this step the Polytec@ LDV, which was pointing at the target surface through an optical path done by mirrors, was aligned as well by using a configuration of mirrors that emulated the one in the tunnel.

5. Fine alignment in the HiRadMat tunnel using pilot pulses: the final alignment took place after the installation of the experiment in the beam line of the HiRadMat facility (TNC tunnel). At the beginning of each irradiation sequence at a given intensity, pilot pulses were sent whilst the sample holder was placed in the no-target position. Then, the whole tank with the BPKG/BTV system attached to it was remotely moved using the motorized experimental tables until the beam was hitting the center of the BTV screen (which had been previously aligned with the target axes in step 2). When the deviation of the beam was small, it was also possible to steer its impact position by the upstream magnet in the line.

With this procedure, it was estimated (based on the accuracy of the laser tracker) that the precision of the position of the targets with respect to the nominal beam axis was within $\pm 0.2 \mathrm{~mm}$. Nevertheless, excitation of bending modes during the experiment and the numerical analysis presented in section 6.5 of Chapter 6 suggest that a misalignment in the order of $0.6 \mathrm{~mm}$ could have been present.

\subsubsection{Operation Procedure}

In this section it is provided a description of the irradiation procedure conceived for the experiment, which is summarized in the flow diagram presented in figure 4.37. The procedure included response to possible abnormal situations in case of non-expected overheating of target rods, tank walls or interferometer heads. It was decided that each of the targets would be impacted by pulses of 7 different intensities, starting from $10^{11} \mathrm{ppp}$ up to $1.5 \cdot 10^{12} \mathrm{ppp}$ (at which AD-Target conditions are recreated as demostrated in section 4.4.1). In this context, it was found much more optimal to irradiate at a fixed intensity the 13 targets before moving forward to the next intensity. In this way, the waiting time necessary for heat removal from each impacted target was optimized. In addition, this strategy was also much more efficient for SPS operation and coordination with the CERN Control Center (CCC) since every change of pulse intensity requires considerable time of optics adjustments and beam tuning.

In this way, the experiment had a main sequence (light blue square in the flow diagram) in which each different target rod was be placed on-beam position to receive a beam impact. After the 13th target was irradiated and provided the proper waiting time to ensure cooling down of the rods, a higher intensity was demanded from the CCC (dark blue square in the flow diagram). 


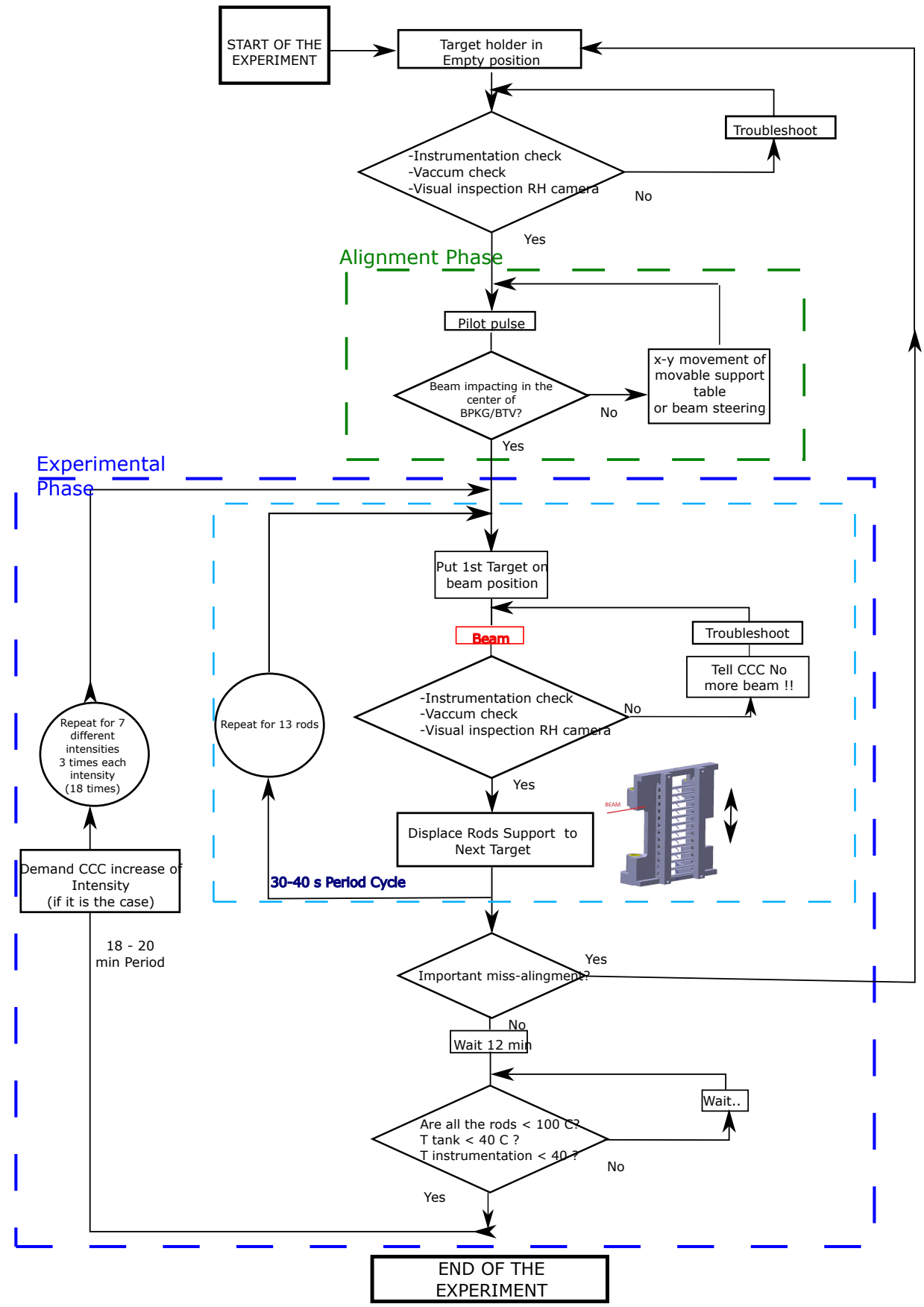

Figure 4.37: Flow diagram of the operation phases during the experiment. 


\subsubsection{Pulses Impacted on Targets}

As already mentioned, the targets were subjected to impacts of proton pulses ranging from low intensity up to the ones which recreated similar conditions as in the AD-Target following an increase of intensity in 7 steps. Table 4.3 shows a summary of all the pulses impacted in the 13 targets. A total of 139 pulses were successfully impacted during the 3 days of experiment. The conditions reached in the targets varied from adiabatic increases of temperature for the low intensity pulses initially estimated in $45{ }^{\circ} \mathrm{C}, 110{ }^{\circ} \mathrm{C}, 130{ }^{\circ} \mathrm{C}$ and $150{ }^{\circ} \mathrm{C}$ for Mo/TZM, Ta, $\mathrm{W} / \mathrm{WLa} / \mathrm{W}-\mathrm{Ta}$ and $\mathrm{Ir}$ respectively. Temperature increases up to approximately $800{ }^{\circ} \mathrm{C}, 1900{ }^{\circ} \mathrm{C}, 2100{ }^{\circ} \mathrm{C}$ and $2250{ }^{\circ} \mathrm{C}$ for Mo/TZM, Ta, W/WLa/W-Ta and $\mathrm{Ir}$ respectively were reached at the highest intensities.

Table 4.3: Summary of irradiation sequences and distribution of the 139 pulses during the experiment

\begin{tabular}{|c|c|c|c|c|c|c|c|c|c|c|c|c|c|}
\hline \multirow[b]{2}{*}{ Total Intensity } & \multicolumn{13}{|c|}{ Impacted pulses per Target } \\
\hline & $\# 1$ & $\# 2$ & $\# \mathbf{3}$ & $\# 4$ & $\# \mathbf{5}$ & $\# 6$ & $\# 7$ & $\# \mathbf{8}$ & $\# 9$ & $\# 10$ & $\# 11$ & $\# 12$ & $\# 13$ \\
\hline$\overline{\text { \# Bunches, bunch space [ns] }}$ & Mo1 & TZM1 & TZM1 & Ta1 & Ta2 & w1 & W2 & W3 & W-La & Ir1 & Ir2 & Ir3 & $\mathbf{W}$-та \\
\hline$\sim 1.00 \cdot 10^{11} \mathrm{ppp}$ & 3 & 2 & 2 & 2 & 2 & 2 & 2 & - & 2 & 2 & 2 & - & 2 \\
\hline 1 bunch & & & & & & & & & & & & & \\
\hline$\frac{\sim 2.15 \cdot 10^{11} \mathrm{ppp}}{12 \text { bunches, } 25 \mathrm{~ns}}$ & 3 & 3 & 3 & 3 & 3 & 3 & 3 & - & 3 & 3 & 3 & - & 3 \\
\hline$\frac{\sim 5.00 \cdot 10^{11} \mathrm{ppp}}{36 \text { bunches, } 25 \mathrm{~ns}}$ & 3 & 3 & 3 & 3 & 3 & 3 & 1 & - & 3 & 1 & 3 & - & 3 \\
\hline$\frac{\sim 7.00 \cdot 10^{11} \mathrm{ppp}}{36 \text { bunches, } 25 \mathrm{~ns}}$ & 1 & 1 & 1 & 1 & 1 & 1 & - & - & 1 & - & 1 & - & 1 \\
\hline$\frac{\sim 2.15 \cdot 10^{11} \mathrm{ppp}}{12 \text { bunches, } 25 \mathrm{~ns}}$ & 1 & 1 & 1 & 1 & 1 & 1 & 1 & - & 1 & 1 & 1 & - & 1 \\
\hline$\frac{\sim 9.20 \cdot 10^{11} \mathrm{ppp}}{36 \text { bunches, } 25 \mathrm{~ns}}$ & 1 & 1 & 1 & 1 & 1 & 1 & - & - & 1 & - & 1 & - & 1 \\
\hline$\frac{\sim 1.27 \cdot 10^{12} \mathrm{ppp}}{36 \text { bunches, } 25 \mathrm{~ns}}$ & 1 & 1 & - & 1 & - & 1 & - & - & 1 & - & 1 & - & 1 \\
\hline$\frac{\sim 1.56 \cdot 10^{12} \mathrm{ppp}}{36 \text { bunches, } 25 \mathrm{~ns}}$ & 1 & 1 & - & 1 & - & 1 & 1 & 9 & 1 & - & 1 & 1 & 1 \\
\hline
\end{tabular}

As observed in the table, there were some targets, such as \#3TZM1, \#5Ta2, 7\#W2, and \#11Ir2, which were irradiated only up to intermediate intensities. The objective was to study during the post irradiation examination the progressive damage in them. It is important to note as well in the table that, after the irradiation at $7 \cdot 10^{11}$, all the targets were subjected to a second irradiation sequence at $\sim 2 \cdot 10^{11} \mathrm{ppp}$. The purpose of this stepdown in intensity was to try and estimate on-line if internal damage on the targets was taking place already by comparing 
these responses with the ones recorded in the previous irradiation at $2 \cdot 10^{11} \mathrm{ppp}$ (before subjecting them to $5 \cdot 10^{11} \mathrm{ppp}$ and $7 \cdot 10^{11} \mathrm{ppp}$ ).

In addition, there were other targets such as \#8W3 and \#12Ir3 which were maintained unirradiated or "virgins" until the end of the experiment, when they were directly exposed to the maximum intensity. The purpose of this was to observe the response of these materials at these extreme loads without having been previously affected by lower intensity pulses. For this reason, one can consider that these targets are the most representative to real AD-Target material response.

\subsubsection{Beam Stability during Operation}

In this subsection a summary of the beam parameters measured during the experiment is presented. These parameters were measured by the beam position monitors (BTV and BPKG) attached upstream the experimental tank as shown in figure 4.17. It must be mentioned that the BPKG did not work properly during the experiment, giving inconsistent beam offsets with respect to the measurements of the BTV and the rest of beam position monitors of the HiRadMat line. These inconsistencies were taking place only when the high density targets were impacted by the beam (not during pilot pulses). For this reason, the malfunction of the BPKG was attributed to back-scattered prompt radiation arriving and affecting the BPKG electronics which, even if placed $35 \mathrm{~m}$ upstream, were not properly shielded. Fortunately, the BTV was fully operative during the whole experiment thanks to the relatively low intensity, large transverse size of the pulses and the short duration of the experiment which prevented screen saturation. The data presented in this section corresponds entirely to BTV measurements.
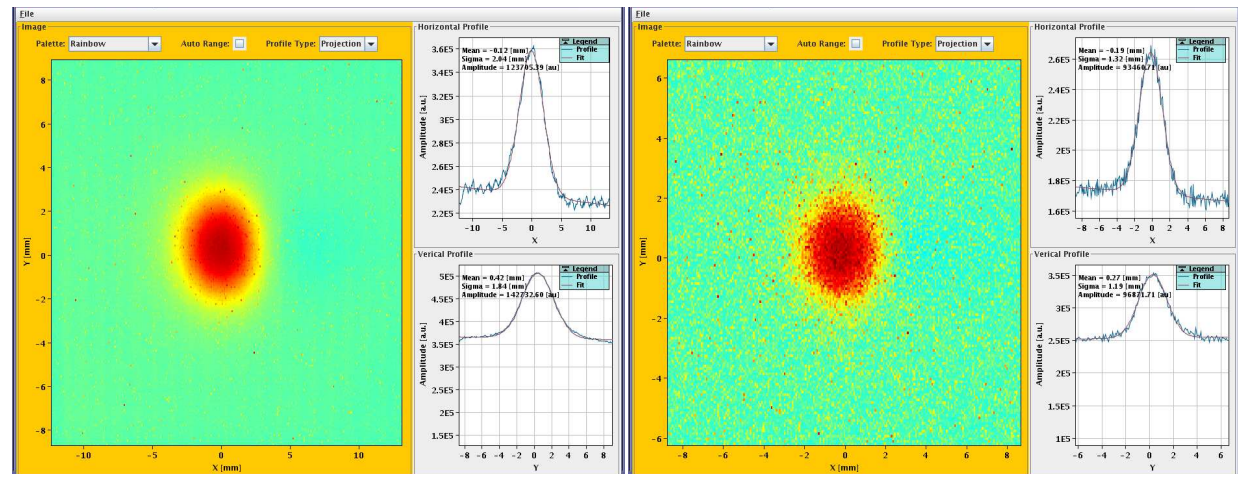

Figure 4.38: Two examples of BTV measurements during the experiment, corresponding to pulses \#7 and \#86, impacted on \#2TZM1 target during irradiation 1 and 4 respectively. Beam sizes and offsets can be easily extracted from these measurements. 
Figure 4.38 shows two examples of BTV measurements recorded during the experiment. The recorded image corresponds to the footprint left by the beam when passing though a scintillating screen. The image is then processed by a program which gives the size and position of the beam based on the detected brightness. It can be seen, for example, that the pulse shown in the left of figure 4.38 had a beam size of $2.04 \mathrm{~mm}$ (horizontal) x $1.84 \mathrm{~mm}$ (vertical) at $1 \sigma$ and its center had an offset of $-0.12 \mathrm{~mm}$ and $0.42 \mathrm{~mm}$ in the horizontal and vertical planes respectively. The center of the BTV was aligned with the target on beam position as described in section 4.6.1, therefore it can be considered that the offset measured by the BTV is equivalent as the impacted in the target.

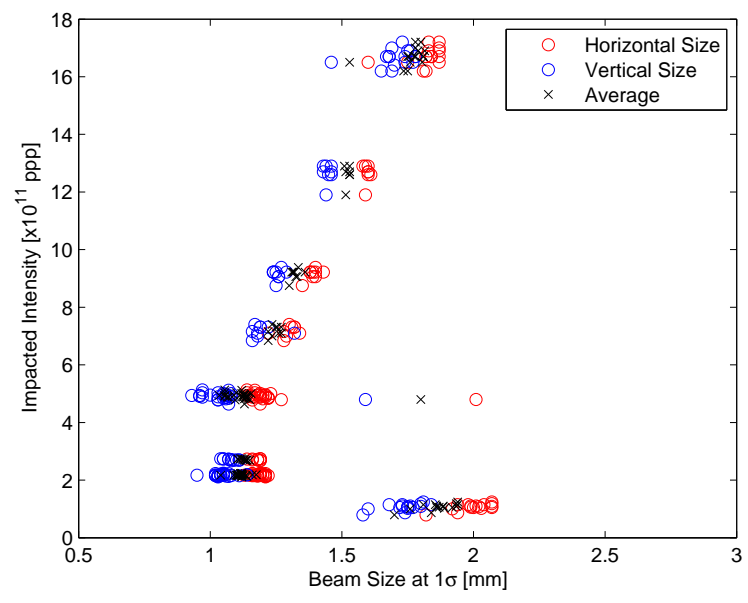

Figure 4.39: Measured horizontal and vertical beam sizes (at $1 \sigma$ ) as a function of intensity for all the impacted pulses during the experiment.

Figure 4.39 gives a summary of the sizes (at $1 \sigma$ ) of all the beams impacted during the experiment as a function of their intensity. The seven staggered irradiation intensities presented in table 4.3 can be noticed. In addition, it can be seen that there were different beam sizes depending on the intensity due to changes and tuning in the beam optics for each irradiation. For example, pulses at first irradiation intensity (consisting in single bunches) had an averaged beam size of $1.8 \mathrm{~mm}$ with a significantly larger scattering in comparison to the pulses at higher intensities (made by 12 or 36 bunches). It can be seen that beam sizes from $1.2 \mathrm{~mm}$ to $1.8 \mathrm{~mm}$ were impacted. These are relatively different than the nominal beam size set in $1.5 \mathrm{~mm}$. Nevertheless, these discrepancies are not expected to influence the results as long as these real beam parameters are taken into account for the experimental-numerical crosscheck (as is done in section 6.4 of Chapter 6). Furthermore, other beam parameters were also adapted on the fly during the 
experiment, such as the intensity for reaching AD-Target conditions, which was set in $1.6 \cdot 10^{12}-1.7 \cdot 10^{12} \mathrm{ppp}$ instead of the nominal $1.5 \cdot 10^{12} \mathrm{ppp}$ to compensate these discrepancies with respect nominal beam size (a more unfocused beam) and therefore deposit a similar peak of energy density in the targets. Finally, another feature interesting is the fact that the vertical beam sizes were in general $0.2 \mathrm{~mm}$ lower than the horizontal, meaning that all the impacted beams were slightly oval.

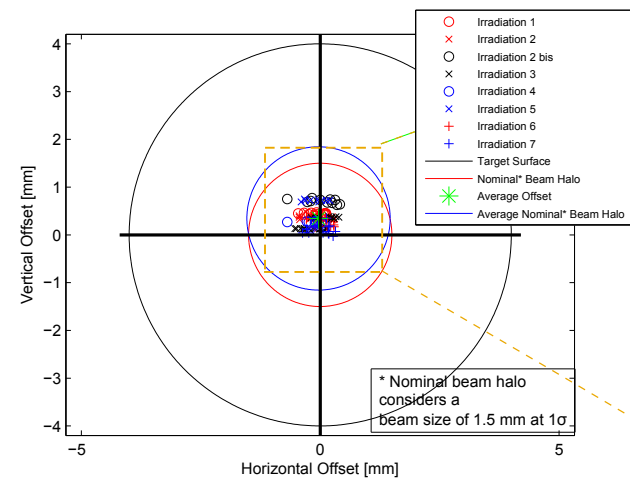

(a)

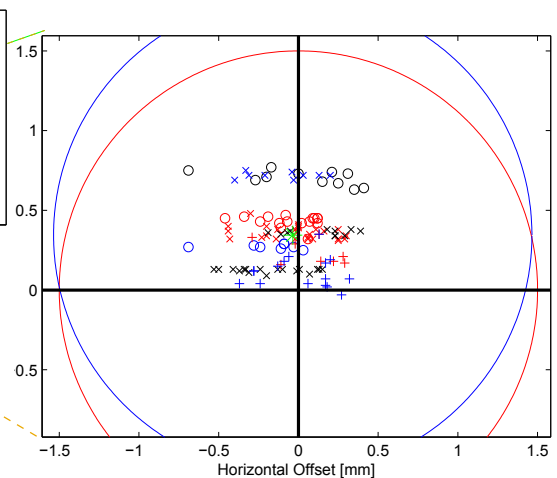

(b)

Figure 4.40: (a) Representation of all the measured positions of impact on the $8 \mathrm{~mm}$ targets during the experiment. (b) Zoomed region of the impacts showing an average offset of $-0.03 \mathrm{~mm}$ and $0.34 \mathrm{~mm}$ in the horizontal and vertical planes respectively.

To complete this analysis, the plots in figure 4.40 show the position of all the impacted pulses during the experiment, taking into account the vertical and horizontal measured offsets. In figure 4.40-(a) a circle representing the geometry of the $8 \mathrm{~mm}$ diameter target is included, giving an idea of the influence that these offsets could have. The representation of the beam halo (taken at $1 \sigma$ ) for the averaged offset is also included. In figure 4.40-(b) the region of the impact is zoomed. Deviation of the impact position from -0.5 to $0.5 \mathrm{~mm}$ in the horizontal plane and from 0 to $0.8 \mathrm{~mm}$ in the vertical took place. The averaged impact position was $-0.03 \mathrm{~mm}$ in the horizontal plane and $0.34 \mathrm{~mm}$ in the vertical, meaning that a systematic deviation of around $0.34 \mathrm{~mm}$ in the positive vertical plane was present. This offset represents around $8 \%$ of the target radius and could be one of the reasons of the observed bending of the targets during the experiment (explained more in derail in sections 5.2 and 6.3 of Chapters 5 and 6 respectively). Another interesting feature that can be observed in figure 4.40-(b) is that for a given intensity (and therefore beam optics) the beam pulse-to-pulse stability in the vertical plane was considerably better than in the horizontal plane, presenting a scattering within the range of only $0.1 \mathrm{~mm}$ in the first, while in the horizontal plane the scattering is in the order of $1 \mathrm{~mm}$. 


\subsubsection{Radiation Levels during Operation}

In this subsection some data regarding online radiation measurements recorded close to the experiment during operation is presented. This data was acquired by the RAMSES (RAdiation Monitoring System for the Environment and Safety) monitor PMIHR02 installed in the HiradMat beam line, around $40 \mathrm{~cm}$ from the experimental table and 1.5-2 $\mathrm{m}$ from the targets. This system uses ionization chambers to measure online radiation dose rate.

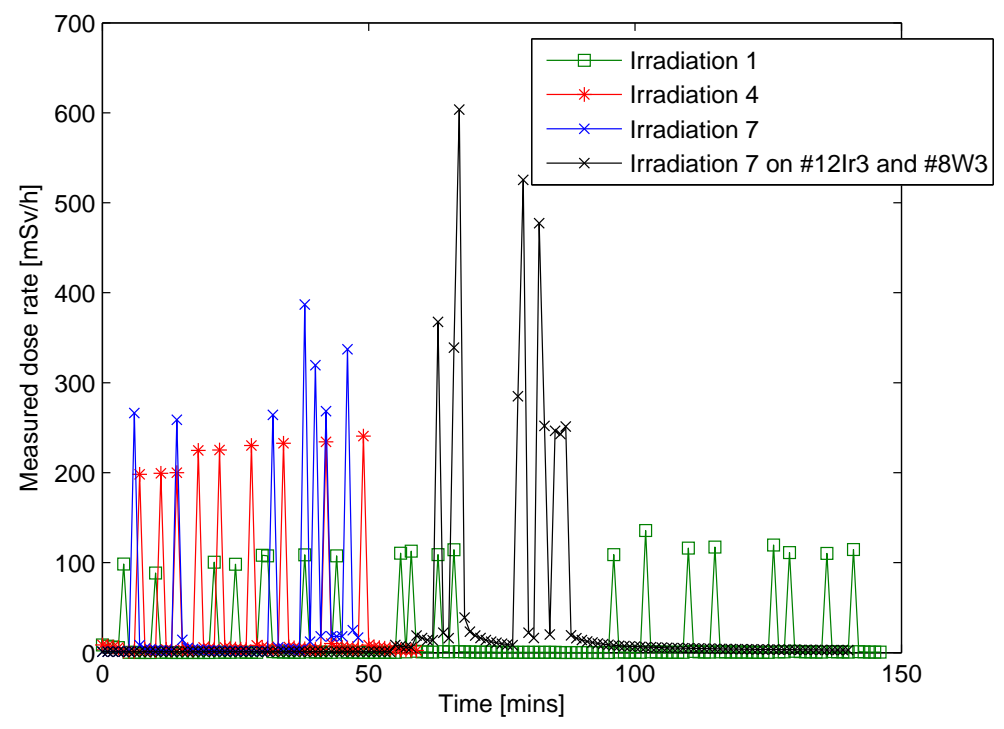

Figure 4.41: 1 min averaged dose rate measured during irradiations 1, 4 and 7 (pulse intensities $1.1 \cdot 10^{11} \mathrm{ppp}, 7.5 \cdot 10^{11} \mathrm{ppp}$ and $1.6 \cdot 10^{12} \mathrm{ppp}$ respectively). It is important to note that the $1 \mathrm{~min}$ averaged does not allow to observe real peaks due prompt radiation as well as that the high intensity measurements may be subjected to saturation due to lack of calibration for mixed particle fields.

Figure 4.41 shows as an example the recoded dose rate as a function of time for the irradiation 1,4 and 7 (pulse intensities $1.1 \cdot 10^{11} \mathrm{ppp}, 7.5 \cdot 10^{11} \mathrm{ppp}$ and $1.7 \cdot 10^{12} \mathrm{ppp}$ respectively). Each of the peak corresponds more or less to an impacting pulse in a target. This data, however, has to by analyzed carefully and only considered as qualitative, since there are several aspects that have to be taken into account; first of all, the acquisition rate presented in the plot corresponds to only 1 minute, this means that the indicated dose rate is an average of the dose rate during this time and therefore is completely unable to catch the prompt radiation peak which takes place in a few nanoseconds. In addition, the radiation monitors are not 
calibrated for the mixed particle fields seen during the operation and depending on the beam intensity the measurements can also be subjected to saturation. Hence, the values presented in the figures 4.41 and 4.42 are not necessarily proportional to the impacted intensity.

The 1 min averaged dose rate explains why no big changes in the measured radiation depending on the density of the target are noticed in figure 4.41. Also, it explains why there is a single peak reaching $600 \mathrm{mSv} / \mathrm{h}$ during the irradiation at highest intensity in targets \#12Ir3 and \#8W3. During this last irradiation, several pulses were impacted in the target \#8W3 spaced by less than 1 min. This peak and the two following ones represent in reality the cumulative dose of these pulses impacted consecutively.

Figure 4.42 shows the maximum 1 min averaged measured dose rate as a function of intensity for all the impacted pulses. As it can be seen in the plot, measured dose increases with impacted intensity. Although this increase does not keep a direct proportion due probably to the saturation just described. In addition, in this plot it can be also observed some variation regarding the measured dose rate for each impacted intensity. This variation can be explained by the difference of targets density and therefore beam-target interaction for a given intensity.

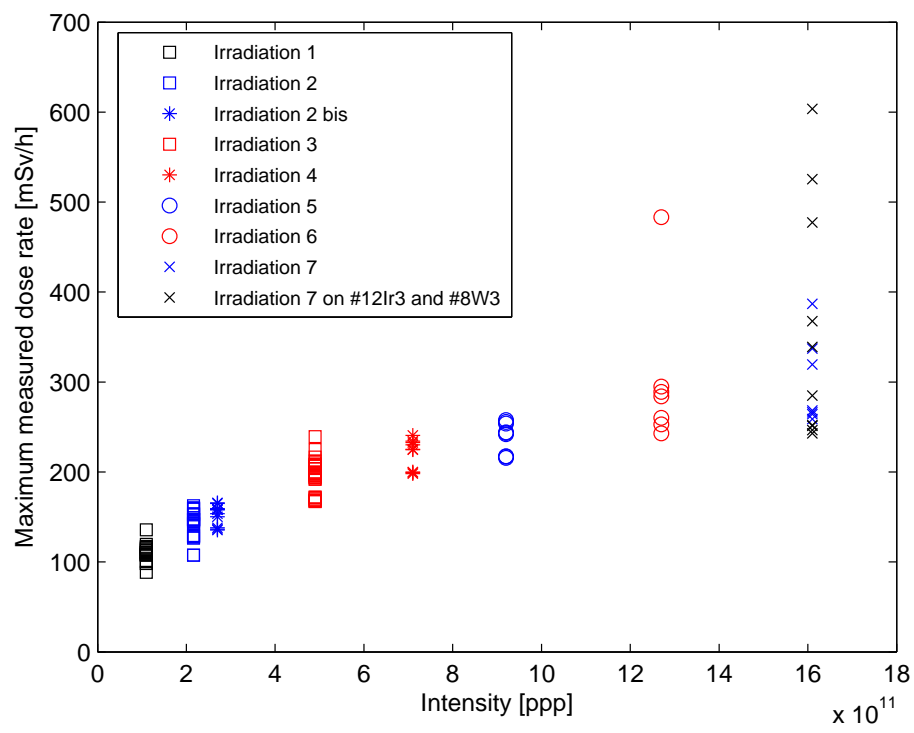

Figure 4.42: (a) Maximum 1 min averaged dose rate recorded during the experiment for all the impacted pulses as a function on the impacted intensity. Similarly as in figure 4.41, some of these values may be subjected to monitor saturation, explaining the lack of direct proportion between dose rate and intensity. 
Another very interesting plot is shown in figure 4.43. This plot was retrieved during the operation of the experiment and for this reason it keeps a much higher acquisition rate than the ones presented in figures 4.41 and 4.42. Therefore, higher and more realistic values of dose rate are presented in this figure (the dose average is done in a smaller time window being closer to the real prompt dose rate). Problem of saturation still applies to this plot though. In any case, the higher precision in the measurement allows to appreciate differences in the recorded dose rate depending on the density of the target and its state at this stage of the experiment. The plot shows the recorded dose rate during the last impacted pulses at the highest intensity (AD-Target conditions). The recorded time allows to identify which target was in the trajectory of the beam, bringing interesting insights.

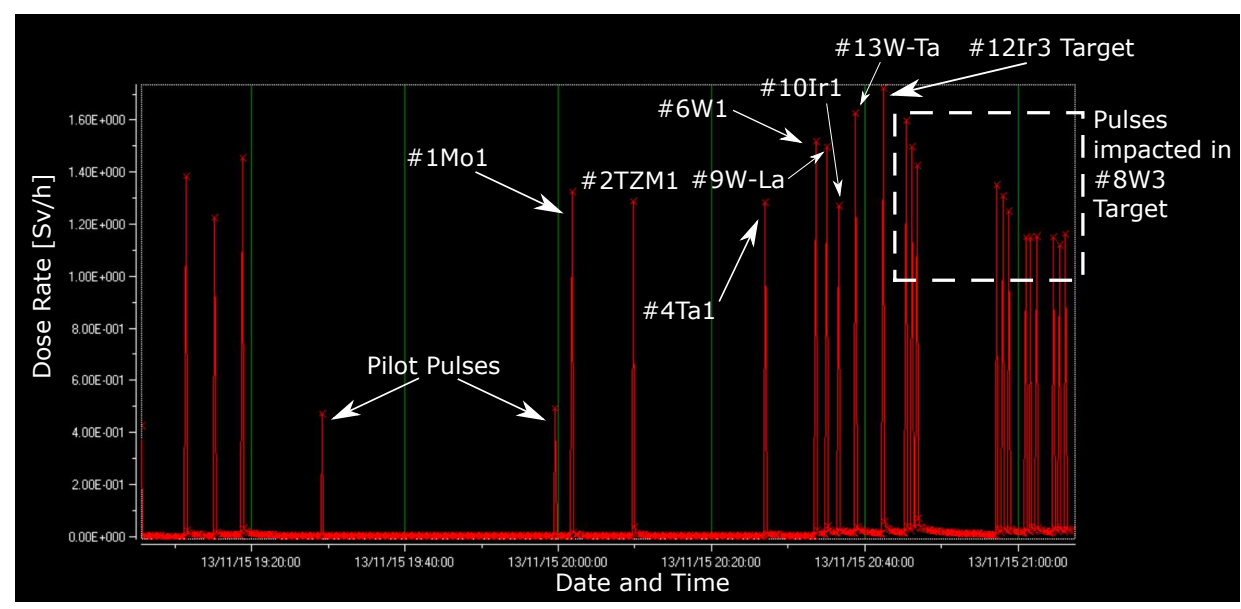

Figure 4.43: Dose rates of the last irradiation sequence (at the highest intensity) retrieved during experiment. Acquisition rate of this plot is significantly higher than $1 \mathrm{~min}$, providing more accurate values than the ones presented in figures 6.1.4 and 6.3. Each corresponding impacted target is identified and indicated in the plot.

For instance, the dose rate measured when the iridium target \#10Ir1 was impacted is considerable lower than the recorded with the tungsten targets \#6W1, \#9W-La (which have lower density) or the "virgin" iridium target \#12Ir3, being the latter the one that produced the highest dose rate $(1.7 \mathrm{~Sv} / \mathrm{h})$. This difference that could initially look like an inconsistency is in reality showing that the beam-matter interaction with the target \#10Ir1 was significantly reduced. The reduction was produced by a considerable target bending due to the precedent beam impacts, as it will be shown in detail in sections 6.1 .4 and 6.3 of Chapter 6. Dose rate recorded with \#4Ta1 is also subjected to this effect. The reduction of beammatter interaction due to bending is even more remarkable when observing the 
last 9 pulses, all of them impacted in the tungsten target \#8W3, which was also unirradiated until then. It can be clearly seen that after the first pulse, reaching $1.6 \mathrm{~Sv} / \mathrm{h}$, the recorded dose rate goes down with each impacted pulse, showing the progressive bending until reaching the state shown in picture 6.6 of section 6.1 .3 in Chapter 6. 



\section{Chapter 5}

\section{The HRMT27 Experiment: Online Results}

In Chapter 4 a presentation of the HiRadMat experiment was provided, including its motivation, goals, involved challenges and finalized design. The experiment intended to impact high intensity and energy proton pulses onto thin rods $(8 \mathrm{~mm}$ diameter and 140-240 mm length) of high-density materials such as iridium, tungsten, molybdenum and tantalum among others. In the experiment, 13 targets of these materials were placed inside a vacuum tank, supported by a vertical linear mobile sample holder capable of putting each of the targets in axis with the incoming proton beam.

The HRMT27 experiment took place in November 2015 using the HiRadMat facility at CERN, impacting 139 proton pulses during 3 days of experiment. A significant amount of online data was successfully recorded. Most of the online instrumentation has performed well, especially the OptoMETR LDV, which was robustly recording during the whole experiment and giving a high quality signal. Due to the distance and difficulties of alignment of the Polytec® LDV, which was placed in the bunker and pointing to the rod on beam position through an optical path guided by mirrors, the signal of this instrument presented high levels of noise. The interferometer on the other hand recorded significant amount of information from the tilted surfaces of the target. The beam position and its parameters were monitored through the BPKG/BTV. It is important to mention that the measurements taken by the BPKG showed strong inconsistencies with respect to the BTV measures and beam parameters measured upstream in the extraction line. These inconsistencies in the BPKG measurements were attributed to perturbation of the electrical signal due to the back-scattered prompt radiation coming from the impacted high density targets, since its DAQ was not shielded and placed far 
enough. Fortunately the BTV worked very well and its recordings on beam position and sizes could be used during the whole experiment without its saturation, thanks to the relatively large beam sizes and short duration of the experiment. Regarding the thermocouples, many of them were detached from the rods due to the high velocities and inertia forces reached. In spite of this (which was somehow expected), at least one thermocouple survived until the end of the experiment in 8 out of 13 targets. The pyrometer recorded only in the high intensity pulses due to its high lower range of acquisition (above $300{ }^{\circ} \mathrm{C}$ on the surface).

In the present chapter, some of the recorded signals resulting from pulse impacts that were particularly interesting will be presented. This data represents only a minor fraction of what it was recorded. However, it was selected in a way which allows to extract a general picture of the behavior of all irradiated materials at the different exposed conditions. With this purpose, just an example of temperature data recorded by the thermocouples and pyrometer is preliminary shown. Following this section, displacement and velocity data recorded mainly by the OptoMET R LDV is presented. This data is shown for the different irradiated materials classified in three different intensity ranges.

- Low intensity pulses: Considering impacted pulses of only $\sim 1 \cdot 10^{11} \mathrm{ppp}$ ( $1^{\text {st }}$ irradiation intensity). This intensity is in the order of $\sim 15$ times lower than the maximum irradiated intensity (at which equivalent conditions of $\mathrm{AD}$-Target are expected). At these intensities, the response of most of materials are still in the elastic regime. This is presented in section 5.2.

- Medium to high intensity pulses: From the $2^{\text {nd }}$ irradiation intensity, $\sim 2$. $10^{11} \mathrm{ppp}$, changes in the response of some of the very high density materials such tungsten or iridium were already observed. In this section, these changes are progressively presented for all the materials up to intensities of $\sim 1.5$. $10^{12}$. The discussion of the progressive internal damage in the targets is done by comparing the response of consecutive pulses impacted in the same target, as well as comparing the different response recorded for each of the materials. This is presented in section 5.3.

- High intensity pulses: In this section two special cases are presented, corresponding to the highest intensity pulses (AD-Target equivalent conditions) impacted onto the iridium and tungsten target that were left "virgin". These are therefore the most representative cases of the conditions reached at the AD-Target. This is presented in section 5.4.

Finally, after the individual presentation of measured signals, plots including relevant quantitative information extracted from many pulses and different materials are presented in section 5.5. These plots summarize most of the recorded data and are very useful for comparing them and withdrawing general conclusions. 


\subsection{Thermocouples and Pyrometer Recordings}

Figure 5.1 shows an example of the target surface temperature recorded by the thermocouples. This case corresponds to the impact of a beam of $1.27 \cdot 10^{12} \mathrm{ppp}$ in a tungsten target. As observed in the plot (b), a maximum temperature of $448{ }^{\circ} \mathrm{C}$ is reached at the point of measurement on the target surface $5.4 \mathrm{~s}$ after the pulse impact. This delay is due to the time needed by the heat to reach the surface from the inner areas of the target where deposited energies and temperatures are much higher. After reaching this peak, the temperature decreased due to heat conductance through the target supports and radiative heat transfer from the surface. After approximately 10 mins, the temperature in the target reached values of $42{ }^{\circ} \mathrm{C}$ as shown in Figure 5.1-(a). It is very interesting as well to differentiate the heat transfer stages taking place during the first instants after the pulse impact. Figure 5.1-(c) shows the temperature during the first $0.8 \mathrm{~s}$. It can be observed that a sudden increase of temperature of $23{ }^{\circ} \mathrm{C}$ occurs during the first $12.5 \mathrm{~ms}$. This is the minimum acquisition time of the thermocouple, meaning that this sudden rise of temperature was probably higher but could not be recorded.

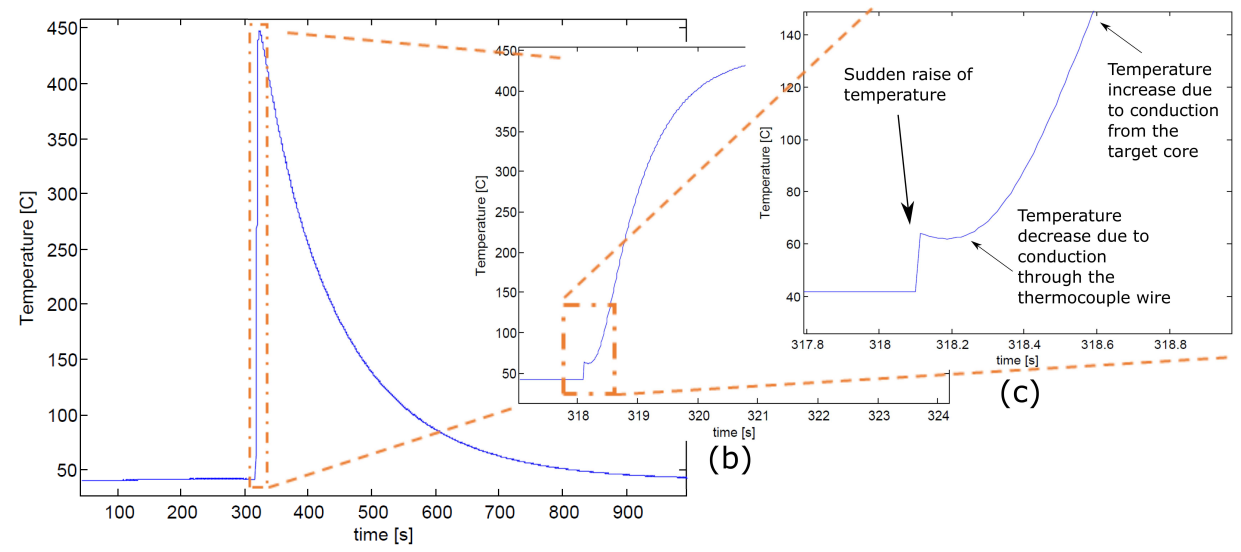

(a)

Figure 5.1: (a) Temperature recorded by thermocouples in a $\mathrm{W}$ target. (b) and (c) shows the recorded temperature during the first $6 \mathrm{~s}$ and $0.8 \mathrm{~s}$ respectively after the proton beam impact.

The cause of this sudden rise of temperature is the energy deposited directly in thermocouple material by the beam halo. After this sudden increase, a second stage can be seen where the temperature slightly decreases a few degrees, reaching a minimum $87.5 \mathrm{~ms}$ after the impact. A possible explanation for this decrease of temperature could be that the heat transfer by conduction through the constitutive metal wire of the thermocouple takes place initially faster than the heat transfer 
from the target material, which is at a higher temperature but does not present a perfect thermal contact with the thermocouple. Finally, after this slight decrease, the measured temperature starts rising due to the heat transfer from the target.

Figure 5.2-(a) shows the temperature recorded by the pyrometer at the target surface during $3 \mathrm{~ms}$ after a beam impact of $1.27 \cdot 10^{12} \mathrm{ppp}$ in target \#8W3. The initial temperature in the target was $520{ }^{\circ} \mathrm{C}$ due to previous impacts. In this figure, it is possible to observe clearly the fast heat transfer from inside the targets to the surface, reaching $652{ }^{\circ} \mathrm{C}$ after $3 \mathrm{~ms}$ of the pulse impact, corresponding to a rise of temperature of $130{ }^{\circ} \mathrm{C}$. The period of acquisition of the pyrometer is $0.25 \mu \mathrm{s}$ allowing, in principle, to record the adiabatic increase of temperature on the target surface after the beam impact as a consequence of the energy deposited by the beam halo (the pulse length is $0.9 \mu \mathrm{s}$ ). However, when observing in detail the recorded temperature during the first $20 \mu \mathrm{s}$ as shown in Figure 5.2-(b), it is possible to see that up to three oscillations of several hundreds of degrees appeared. The origin of these oscillations is still unexplained. A possible explanation could be that they are just noise caused by an electromagnetic pulse created during the impact and affecting the electronics (this phenomenon was clearly observed in the LDV readings). Nevertheless, after these oscillations, it can be observed that a rise of temperature of $25^{\circ} \mathrm{C}$ is taking place within the first $20 \mu s$ after the impact.

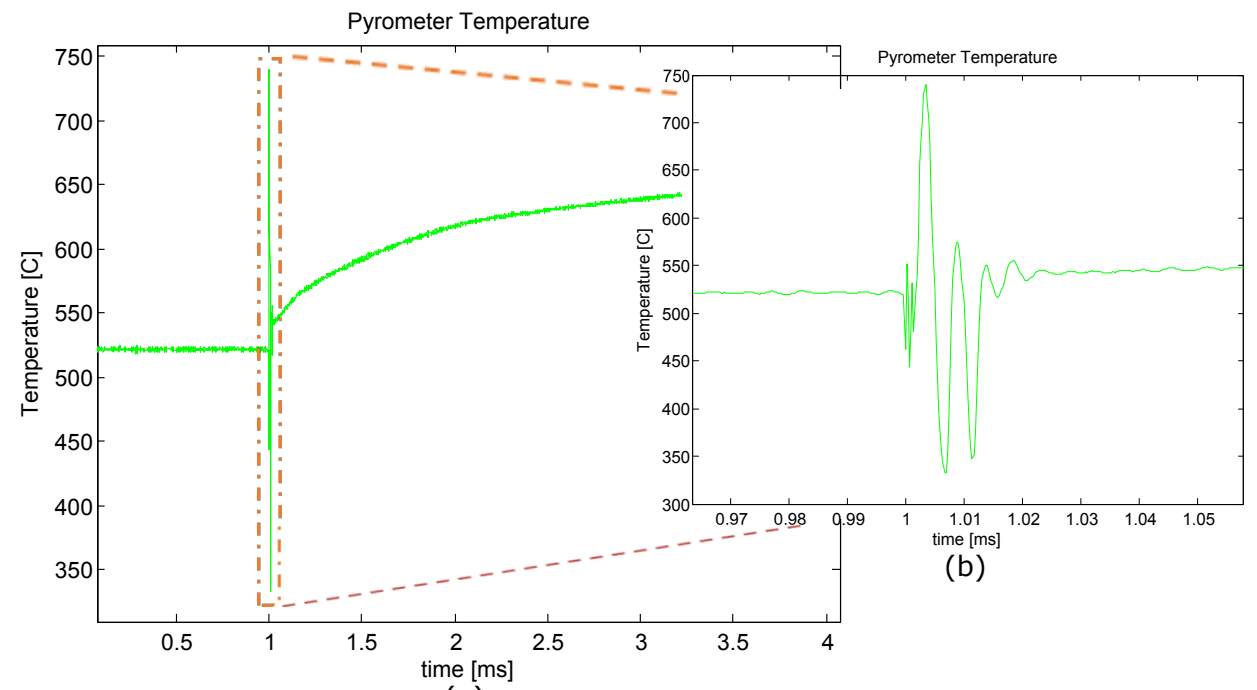

(a)

Figure 5.2: (a) Temperature recorded by the pyrometer at the \#8W3 target surface when impacted by $1.27 \cdot 10^{12} \mathrm{ppp}$. 


\subsection{Dynamic Response at Low Intensity Pulses}

In this section the recorded velocities and positions of a few pulses are presented in order to show interesting wave phenomena that were identified at the low intensity pulses. In addition, differences between material responses were already observed even at these low intensities. Figure 5.3 shows the measured velocity by the LDV OptoMETR on a TZM target after an impact of $1.15 \cdot 10^{11} \mathrm{ppp}$. The LDV, as the pyrometer, was measuring during $3 \mathrm{~ms}$ after the impact. It can be seen in the figure that the response on velocity presents a very low level of damping since the TZM material is still behaving in the elastic regime. This will be shown as well by Figure 5.8. Figure 5.3-(b) shows the velocity response during the first $70 \mu s$ after the pulse impact. As predicted by the calculations described in Chapter 3, it can be observed that high frequency radial waves with periods around $1.8 \mu$ s dominate the response. The periods of these radial waves or modes were also analytically predicted in section 2.2.2 of Chapter 2 .

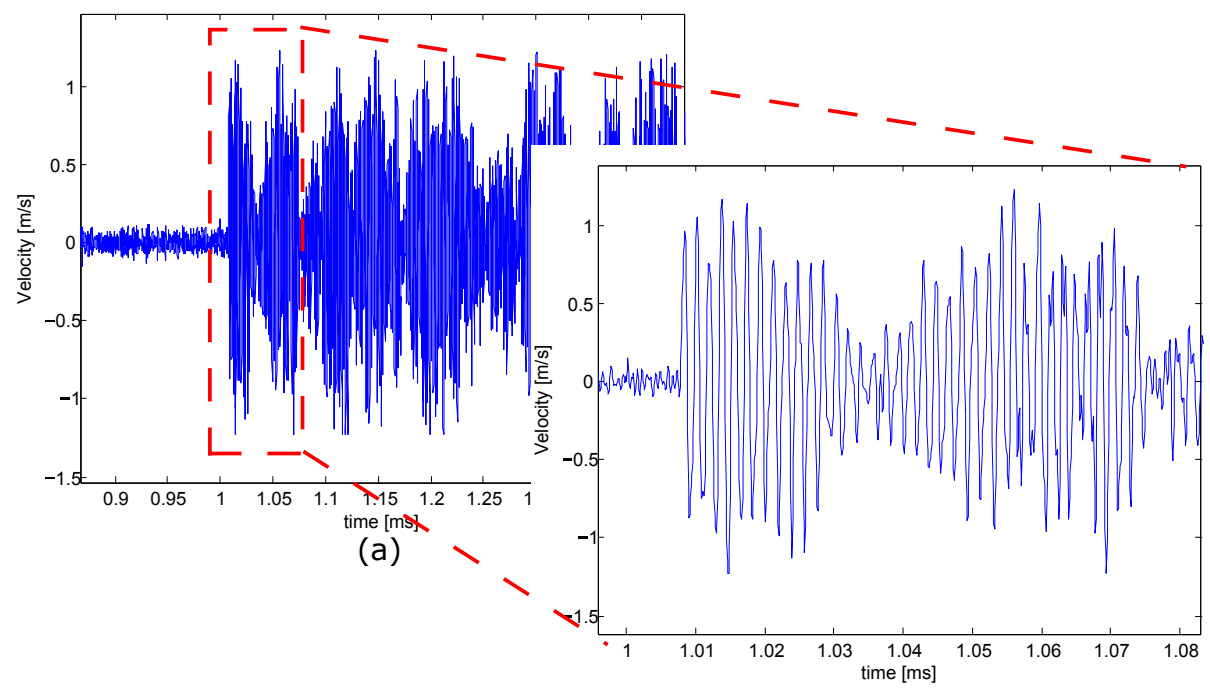

(b)

Figure 5.3: Velocity at the surface of target \#2TZM1 when impacted by $1.15 \cdot 10^{11} \mathrm{ppp}$ (elastic regime) recorded by the LDV OptoMET®

This radial wave dominance can be clearly observed in figure 5.4-(a), where a Fourier transformation has been applied to the signal. It can be seen that a peak at $555 \mathrm{kHz}$ dominates the frequency domain of the recorded velocity. It is interesting to remark that there is not single radial wave but a combination of many of them with slightly difference frequencies. This effect can be noticed in velocity 
response shown in figure 5.3-(a), in which several peaks in the velocity spaced by $\sim 100-130 \mu s$ are present. This is the typical shape of an amplitude modulated wave also known as "beat" in acoustics. This effect is caused by the superposition of two or more waves with similar amplitude and slightly different frequencies, which lead to a much lower frequency modulation of the overall wave envelope due to the alternation of their interference between "in phase" and "out of phase". From the $1.8 \mu$ s period $(550 \mathrm{kHz})$ of the dominant radial response observed, and the $\sim 100-130 \mu$ s period of the modulated one, it can be estimated that in reality there are several radial waves (or modes) with periods oscillating in the range of $1.75 \mu \mathrm{s}-1.85 \mu \mathrm{s}$. This can be also observed in the Fourier transformation of figure $5.4-(\mathrm{a})$, in which the peak at $550 \mathrm{kHz}$ is not isolated but surrounded by components from $540 \mathrm{kHz}$ to $550 \mathrm{kHz}$.

Origin of these different radial waves with slightly different frequencies is unknown but it can be claimed that they are related to the intrinsic generation and propagation of the radial waves, as this modulation also appears in simulations, even if assuming an infinitely elastic material. Local geometry effects such as the presence of the flat surface for the LDV measurement are not the cause either, since these beats have been also observed in simulations considering a perfect cylindrical geometry. A possible explanation could be the excitation of multiple radial modes which are sightly out of phase due to the fact that the energy of the proton beam is deposited in a discontinuous manner, since it is composed by multiple bunches.

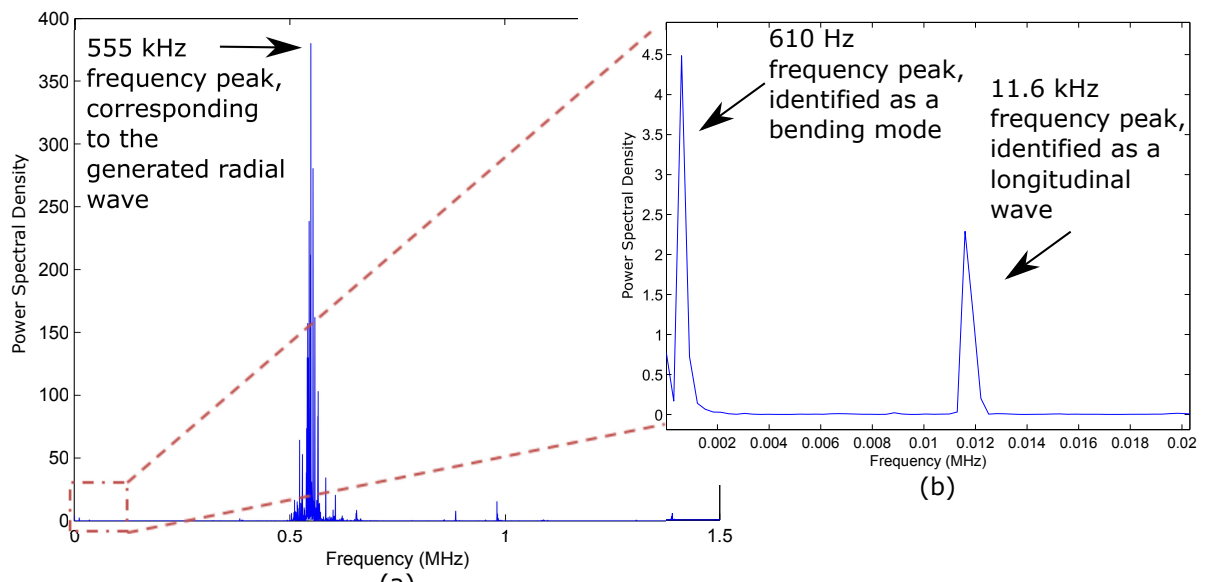

(a)

Figure 5.4: (a) Plot of frequency domain of the surface velocity of target \#2TZM1 when impacted by $1.15 \cdot 10^{11} \mathrm{ppp}$ (elastic regime). The plot shows how a radial wave with a frequency of $550 \mathrm{kHz}$ dominates. (b) Zoom of the lower frequency range where two other waves can be observed, at $610 \mathrm{~Hz}$ and $11.6 \mathrm{kHz}$. 
Similarly as in the Fourier analysis shown in figure 5.4-(a), figure 5.5 which shows the high frequency domain of the recorded velocity at low intensity pulses for the seven irradiated materials. It can be seen that the period corresponding to the dominant radial wave $(1.8 \mu \mathrm{s}$ for the case of Mo and TZM) changes for each of the irradiated material depending on their properties (young modulus, Poisson's ratio and density). These experimental measurements are fully consistent with the ones analytically predicted and presented in table 2.1 of section 2.2.2 of Chapter 2. In $\mathrm{W}$ and $\mathrm{W}$-La the radial wave presented a period of $2.32 \mu \mathrm{s}$, while in Ir and Ta of $2.22 \mu \mathrm{s}$ and $2.78 \mu \mathrm{s}$, respectively. The speed of sound in the Ta is the slowest one due to its considerable smaller bulk modulus in comparison with the rest of the materials. The radial wave of the tungsten target cladded in tantalum (W-Ta) has the higher period $(3.2 \mu \mathrm{s})$ since the total diameter of this target is $12 \mathrm{~mm}$ instead of $8 \mathrm{~mm}$. The frequency domain of the recorded velocity of the W-Ta cladded target presented in figure 5.5 shows also two different peaks at higher frequencies, most probably due to wave reflections at the $\mathrm{W} / \mathrm{Ta}$ interfaces.

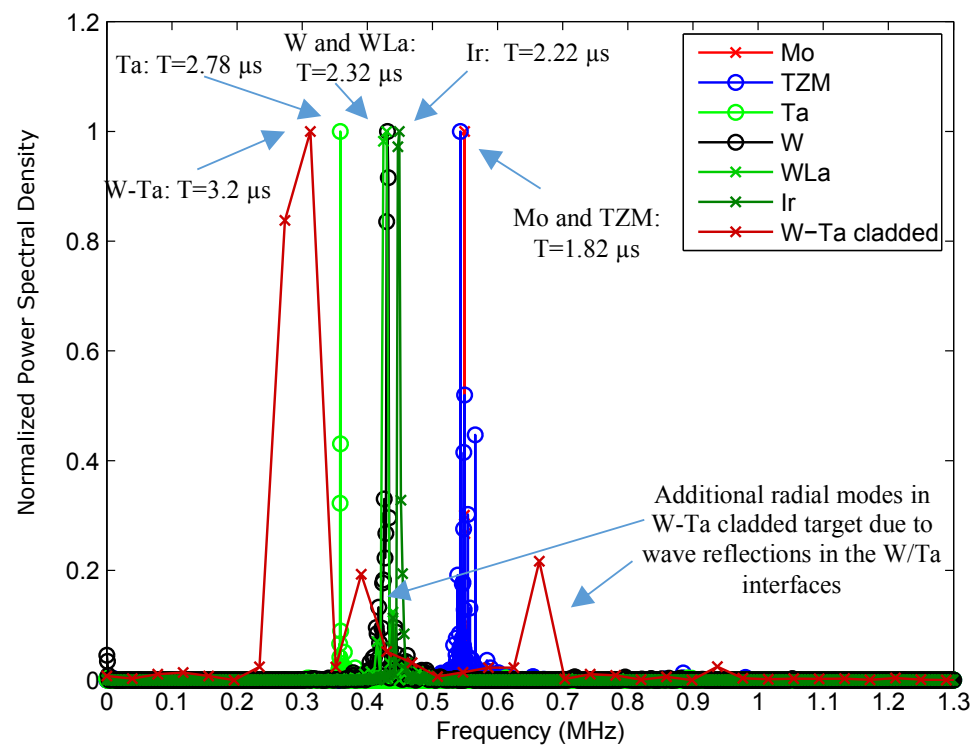

Figure 5.5: Summary plot of the frequency domains of the surface velocity recorded at low intensity pulses for the seven irradiated materials. Different periods of the recorded dominant radial wave consistent with the different speeds of sound in each material can be observed.

Figure 5.6-(a-c) shows the displacement response of the same TZM target as in figure 5.3, obtained from the integration of the measured velocity. In these figures, 
it can be seen that the target response is composed by three clearly differentiated waves: radial, longitudinal and bending mode. These three waves (or modes) can be observed as well in the frequency domain of the recorded displacement presented in figure 5.7-(a-c). It is important to note that the amplitudes of these three components of the frequency domain of displacement are significantly different to the ones of the velocity response showed in figures 5.4 and 5.5 since, from the point of view of displacement, the bending mode dominates followed by the longitudinal and radial wave. In the next paragraphs these three components are analyzed in detail.
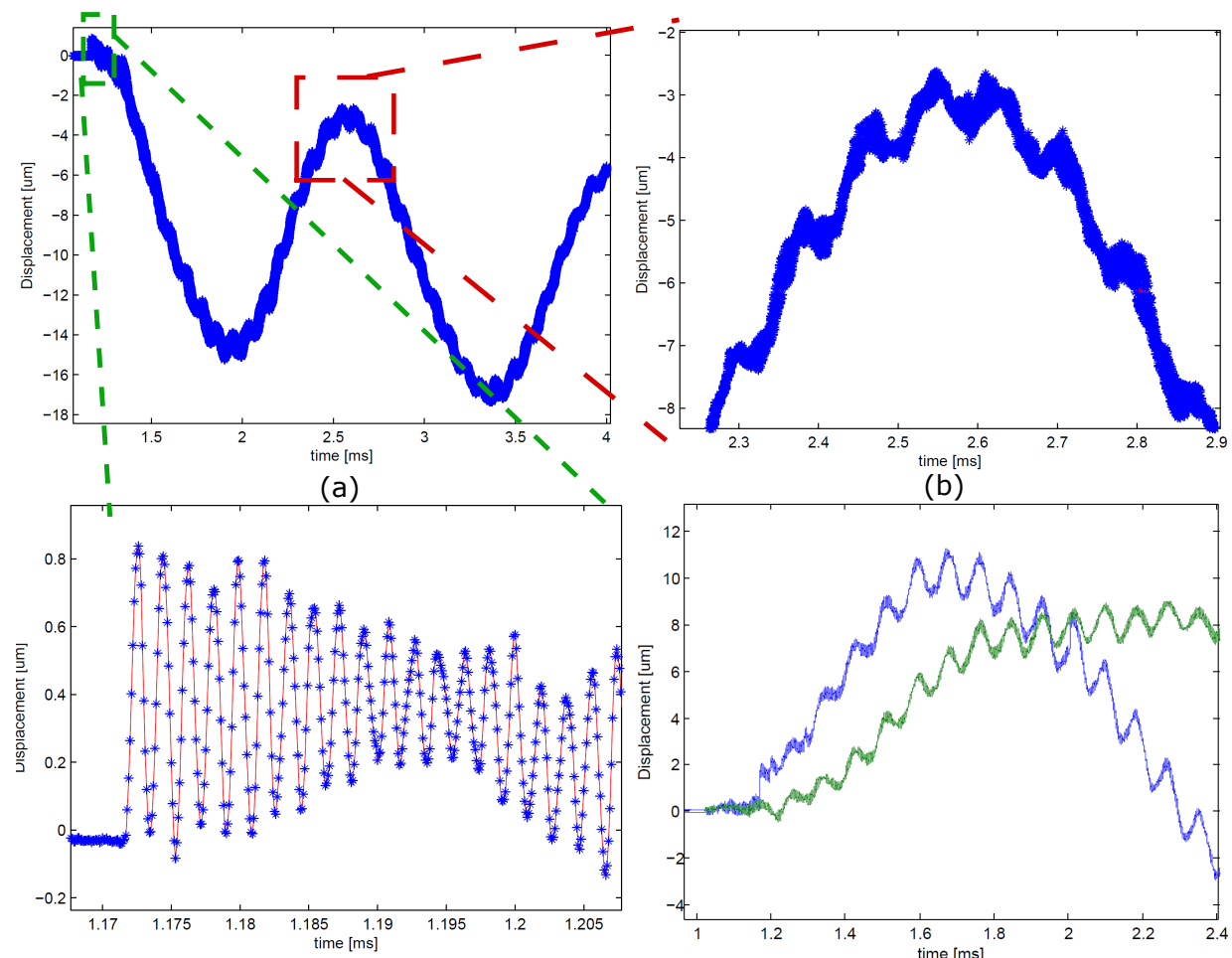

(b)

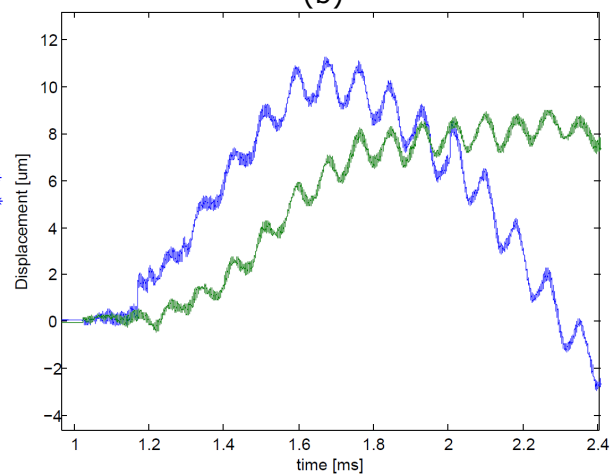

(d)

Figure 5.6: (a-c) Displacement response recorded by the LDV when impacting \#2TZM1 with $1.15 \cdot 10^{11}$ ppp. Bending modes wave, longitudinal waves and radial waves can be identified in (a), (b), (c) respectively. (d) Displacement recorded by the interferometer where the longitudinal waves can be identified as well (blue $=$ Interferometer 1 , Green $=$ Interferometer 2).

I In figure 5.6-(c) is shown the response during the first $40 \mu$ s after the beam impact. The radial wave recently described can be observed. For TZM and Mo under these conditions the radial wave is not damped, and it is still present even $3 \mathrm{~ms}$ after the impact and more than a thousands of oscillations. 
II In figure 5.6-(b) the displacement response in a window of $700 \mu$ s is presented, showing several oscillations of the longitudinal wave analytically predicted in section 2.14 of Chapter 2, as well as in the simulations of Chapter 3. This wave can be seen also in the frequency domain of figure 5.7-(b). This longitudinal wave has a period around $\sim 87 \mu$ s for Mo and TZM as predicted in table 2.2 and it was especially observable in these targets up to high ranges of irradiated intensities, due mostly to the more elastic response of these materials caused by their lower density and less deposited energy. Figure 5.6-(d) shows the surface displacement recorded by the interferometer in the inclined planes machined on the target. It can be observed that these longitudinal and radial waves were measured as well by this device.

III The third recorded wave presents a much lower frequency, as noticed in figure 5.6-(a) and figure 5.7-(a). This kind of wave, with a period of $\sim 1.64 \mathrm{~ms}$ $(610 \mathrm{~Hz})$, involves large displacements in comparison with the radial and longitudinal ones, and it has been observed for all the targets irradiated during the experiment at all the intensities (with significant changes in the period when increasing intensity). The origin of this wave is a bending mode caused by small offsets in the beam impact position and asymmetries in the clamping system of the targets. These bending modes appearing in rods impacted by proton beams have been identified and described previously in the literature (ref. [146]) as well as in the section 2.2.2 of the present work. Although in the study of ref. [146], the authors considered much larger off-axis impacts (0.6 times the radius) compared with the high levels of accuracy in the beam impacts achieved in this experiment (maximum eccentricities were in the order of $0.5 \mathrm{~mm}$ ). It could be the case, however, that even with high level of accuracy expected in the experiment, small offsets in the impact position were enough to excite these modes. In addition, these bending modes are strong candidates to explain the large levels of bending and deformations observed in all the targets at the end of the experiment and described in detail en section 6.3 of Chapter 6 , since the influence of these initial small offsets was cumulative and progressively amplified by the permanent bending deformation of the targets when successively impacted. The numerical prediction of this mode by hydrocode simulations is not straight forward in practice since it requires extremely long transient simulations to reach this low frequency. Furthermore, these modes are very sensible to the uncertainties on the real state of the spring clamping systems of the targets, which are difficult to recreate as boundary conditions and contact algorithms in Autodyn $\mathbb{R}$. This is why, a numerical analysis of the origin of these lower frequency waves has been performed using conventional ANYSY $®$ mechanical and it is presented in section 6.5 of Chapter 6 . 


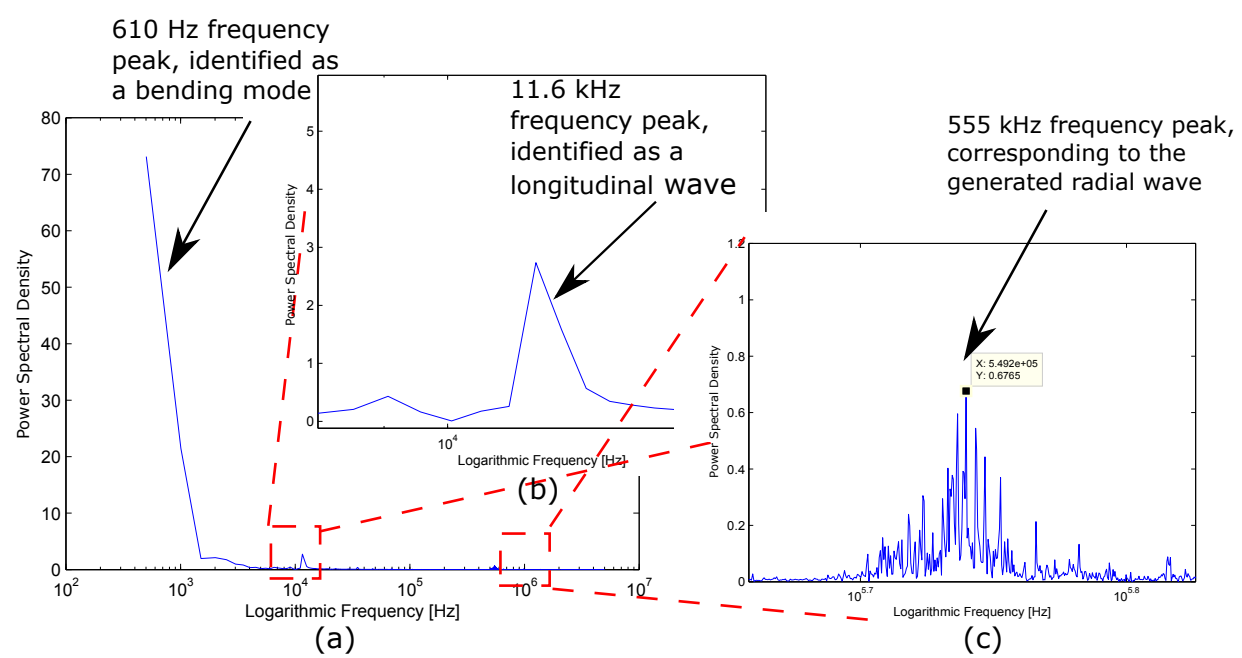

Figure 5.7: (a) Plot of frequency domain of the surface displacement of target \#2TZM1 when impacted by $1.15 \cdot 10^{11} \mathrm{ppp}$ (elastic regime). (b) and (c): Zoom of the higher frequency range where the longitudinal and radial wave can also be observed. Note the big difference in amplitudes in displacement in comparison with the frequency domain of velocities shown in figure 5.4.

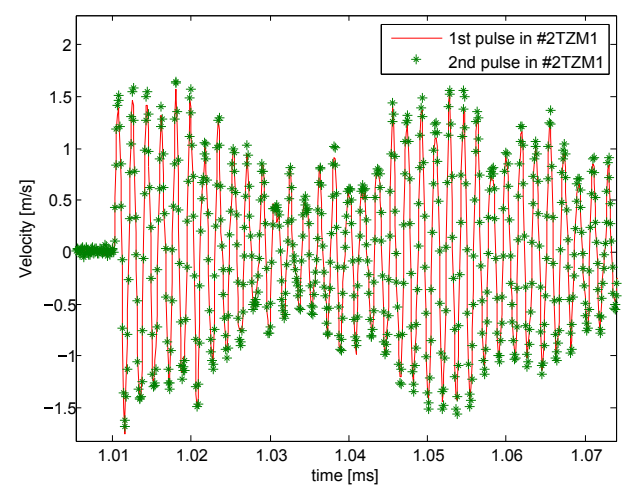

(a)

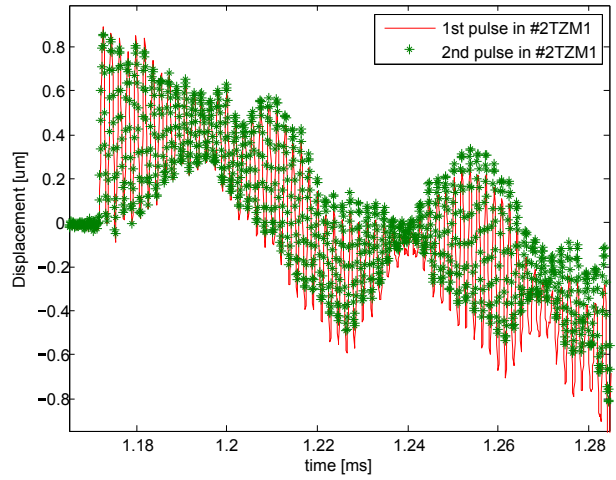

(b)

Figure 5.8: Comparison between recorded responses of two consecutive impacted pulses on \#2TZM1 at the lowest intensity $\left(1.15 \cdot 10^{11} \mathrm{ppp}\right)$. (a) Velocity recorded. (b) Position recorded.

As previously stated, important differences between the irradiated materials were observed even for the low intensity pulses. Figure 5.8 (a) and (b) show the comparison of the velocity and position response of the \#2TZM1 target when impacted by two successive low intensity pulses (red line is first pulse while green dots is the 
second). It is very interesting to see that the response produced by two pulses is almost the same, without almost any shift in the frequency or velocity (figure 5.9). This clearly indicates that the material responds elastically and the beam has not produced internal changes in the target. On the other hand, figure 5.10 shows a similar comparison between two consecutive low intensity pulses but in a tungsten target. First of all, it can be seen how the tungsten velocity response (figure 5.10) is very different compared to the one of TZM (figure 5.3). The surface velocity of tungsten is rapidly damped before $1 \mathrm{~ms}$. In addition, it can be observed in the comparison of the velocity between the first and the second impacted pulses that damping occurs even faster in the second. Looking in detail to the first $7 \mu \mathrm{s}$ after the pulse impact shown in figure 5.10-(b), it can be seen that a shift and change of period of the radial wave takes place after a few oscillations. All these hints clearly indicate that the tungsten material is already experiencing plastic deformation even for low intensity pulses $\left(1.09 \cdot 10^{11} \mathrm{ppp}\right)$. In any case, a clear radial wave can be still identified, suggesting that significant internal cracking is not taking place yet.

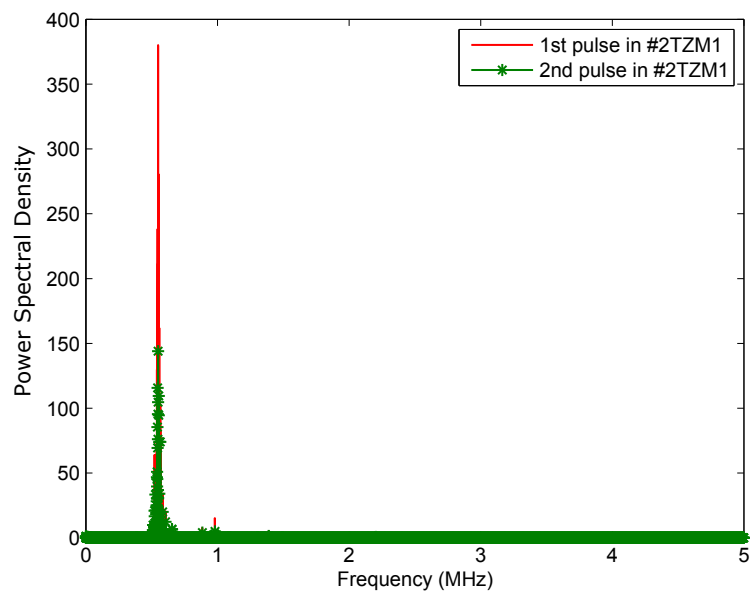

Figure 5.9: Comparison in the velocity frequency domain between recorded responses of two consecutive impacted pulses on \#2TZM1 at the lowest intensity $\left(1.15 \cdot 10^{11} \mathrm{ppp}\right)$. The plot suggests that permanent changes in this target material are not taking place yet (elastic regime) since the frequency of the recorded radial wave is the same. 


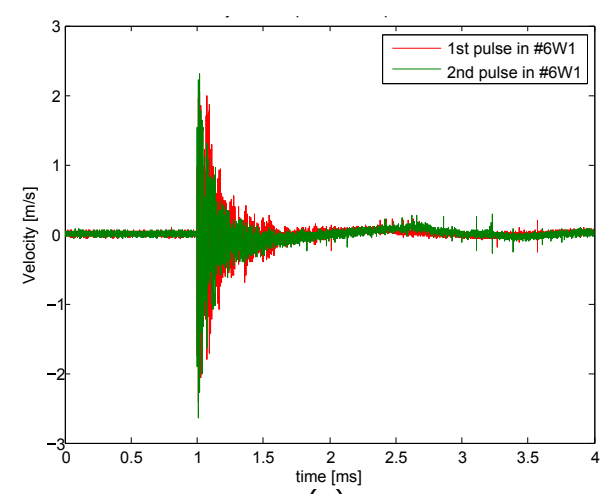

(a)

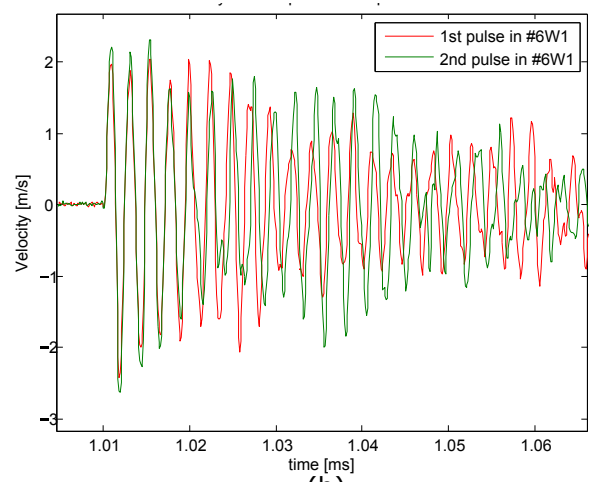

(b)

Figure 5.10: Comparison between recorded responses of two consecutive impacted pulses on $\# 6 \mathrm{~W} 1$ at the lowest intensity $\left(1.09 \cdot 10^{11} \mathrm{ppp}\right)$. (a) Velocity recorded during $3 \mathrm{~ms}$ (b) Detailed velocity during the first $60 \mu \mathrm{s}$.

Figure 5.11 shows the same comparison between the first two consecutive pulses but for iridium. As can be seen in the figure, larger velocities compared to the ones reached in TZM and tungsten were recorded, due to iridium higher density, interaction with the proton beam and subsequent deposited energy. Its response however seems to be less rapidly damped when compared with the tungsten one, as it takes approximately $2 \mathrm{~ms}$ to damp the velocity response after the first pulse. In any case, it can be observed in figure 5.11-(a) that the first pulse already produces plastic deformation in the material, but less pronounced as in tungsten since the radial response in the first and second pulse are clearly similar during a large number of oscillations, as shown in figure 5.11-(b).

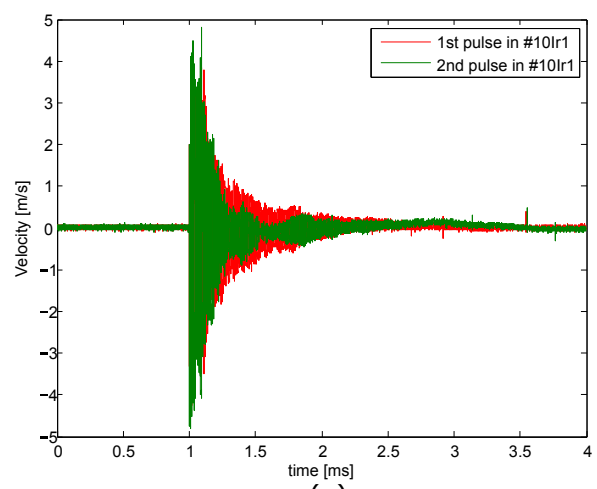

(a)

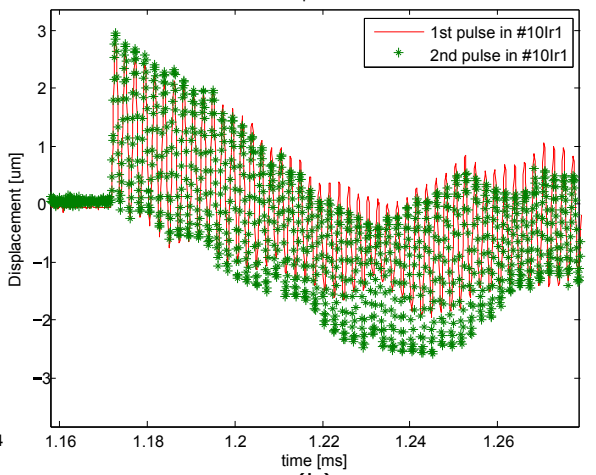

(b)

Figure 5.11: Comparison between recorded responses of two consecutive impacted pulses on \#10Ir1 at the lowest intensity $\left(1.08 \cdot 10^{11} \mathrm{ppp}\right)$. (a) Velocity recorded during $3 \mathrm{~ms}$ (b) Detailed radial displacement during the first $60 \mu \mathrm{s}$. 


\subsection{Change of the Dynamic Response when Increasing Intensity}

In this section, a discussion of the recorded response of the targets while progressively increasing the intensity is presented. The pulses at the intensities in which important changes in the dynamic response were identified are presented for the different materials.

\subsubsection{Targets Experiencing Plastic Deformation and Internal Damage}

Figure 5.12(a)-(b) shows the velocity response measured by the LDV when impacted by three consecutive pulses at $2.11 \cdot 10^{11} \mathrm{ppp}$ ( $2^{\text {nd }}$ irradiation intensity) for (a) tungsten and (b) iridium, respectively. These plots show the velocity only during the first $50 \mu$ s after the impact since after this time the velocity is almost completely damped. It can be observed how the response in both of the targets radically change in comparison with the ones shown in figures 5.10-(b) and 5.11-(a). Even the first pulse at this intensity (red) produced a fast damped and distorted radial wave. This effect is even more accentuated with the following pulses (green and blue). It can be seen how the maximum velocity reached in the tungsten target in the next pulses progressively decreases, reaching a maximum value of $4 \mathrm{~m} / \mathrm{s}$ in the third consecutive pulse while in the first one was $6 \mathrm{~m} / \mathrm{s}$. This could clearly indicate an internal damage and cracking in the material below the mirror of LDV measurement. It is interesting to compare this response with the one observed in the simulations when assuming a failure model as it was shown in figure 4.7-(b) of Chapter 3. The iridium (figure 5.12-(b)) behaves better than the tungsten, but in any case it can be observed how the radial wave starts to be distorted, in particular after some initial oscillations during the $3^{\text {rd }}$ consecutive impacted pulse. It is important to note at this point that the conditions reached at these intensities are still in the order of 7 times lower than the reached in the real AD-Target, i.e, the impacted intensity of $2.11 \cdot 10^{11} \mathrm{ppp}$ is estimated to produce an adiabatic temperature rise of only $300{ }^{\circ} \mathrm{C}$ (in comparison with the $2000{ }^{\circ} \mathrm{C}$ estimated in the AD-Target) and tensile pressures of - $0.8 \mathrm{GPa}$ (in comparison with the $-5 \mathrm{GPa}$ of AD-Target). 


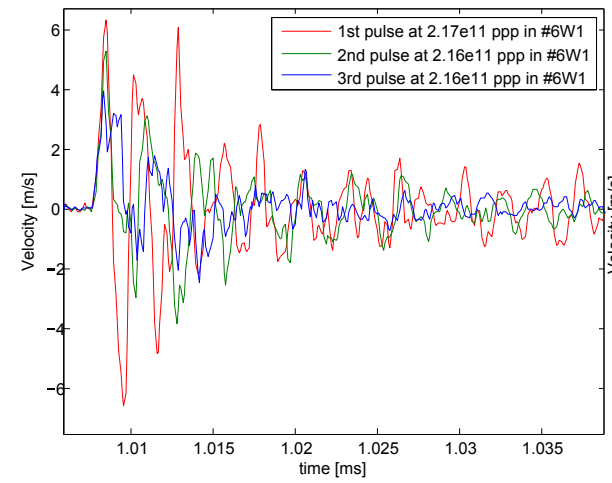

(a)

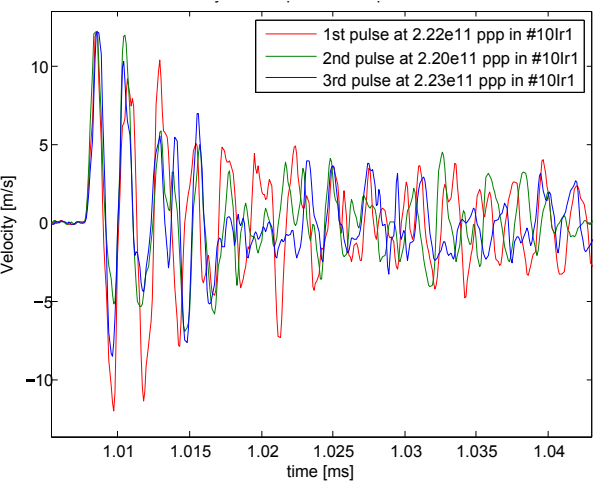

(b)

Figure 5.12: (a) Recorded surface velocity in \#6W1 target after being impacted three consecutive times by a beam of $2.11 \cdot 10^{11} \mathrm{ppp}$. (b) Recorded surface velocity in \#10Ir1 target after being impacted three consecutive times by a beam of $2.11 \cdot 10^{11} \mathrm{ppp}$. Changes in the frequency and a distorted radial wave can be observed in both cases, suggesting internal damage in the inner target material already at these conditions.

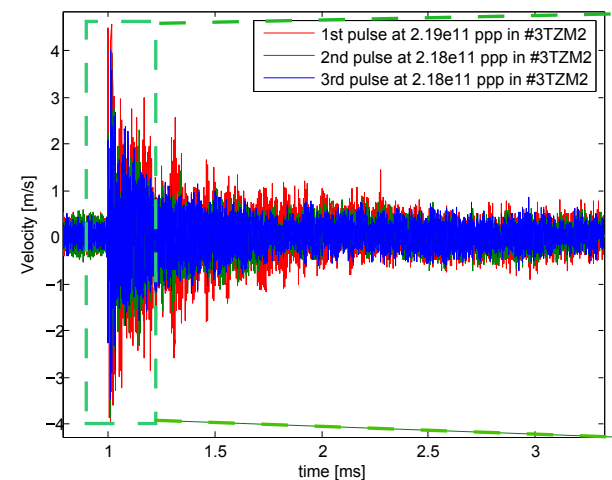

(a)

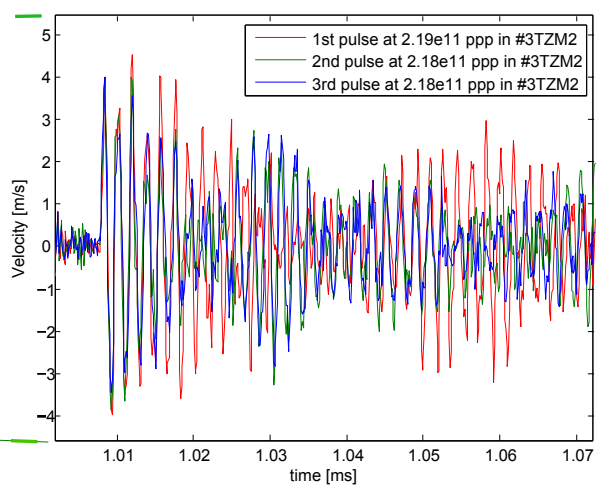

(b)

Figure 5.13: Recorded surface velocity in \#3TZM2 target after being impacted three consecutive times by a beam of $2.18 \cdot 10^{11} \mathrm{ppp}$. (b) Zoom of the recorded velocity during the first $60 \mu \mathrm{s}$. Changes in the radial wave suggest that the material is experiencing plastic deformation.

In a similar way, figure 5.13(a)-(b) shows the surface velocity of a TZM target when impacted by a beam of $2.2 \cdot 10^{11} \mathrm{ppp}$. The target shows a better response than tungsten and iridium but in any case it can be seen in figure 5.13(b) that the radial wave starts to be distorted after some oscillations generated by the $3^{\text {rd }}$ impacted pulse. This could indicate already that plastic deformation and changes in target material are taking place. Figure 5.14 shows the velocity response of the 
same TZM target but at a higher step of irradiated intensity $\left(4.95 \cdot 10^{11} \mathrm{ppp}\right)$. It can be observed that a faster damping of the wave takes place and lower maximum velocities are progressively reached even if the impacted intensity is double than in the previous case. In any case, the response of this TZM target at this higher intensity is still better than the one of tungsten and iridium at $2.2 \cdot 10^{11} \mathrm{ppp}$.

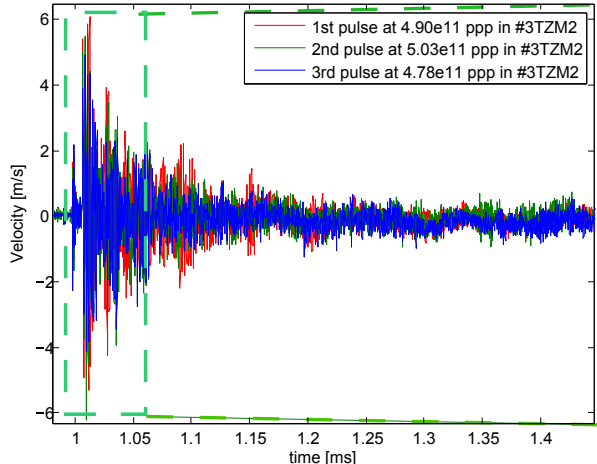

(a)

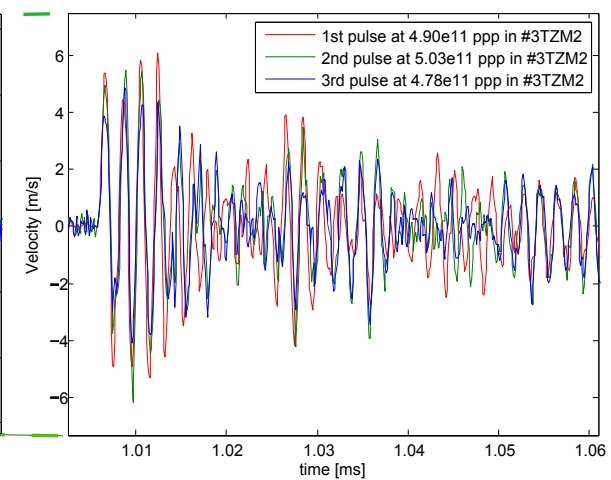

(b)

Figure 5.14: Recorded surface velocity in \#3TZM2 target after being impacted three consecutive times by a beam of $\sim 5 \cdot 10^{11} \mathrm{ppp}$. (b) Zoom of the recorded velocity during the first $50 \mu \mathrm{s}$. The damping of the wave and distorted response is accentuated.

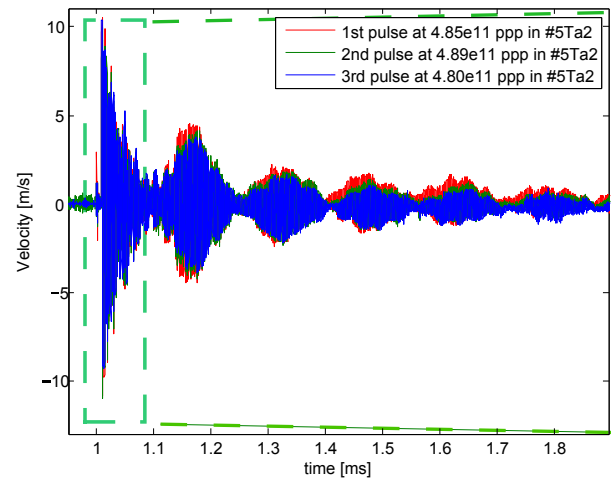

(a)

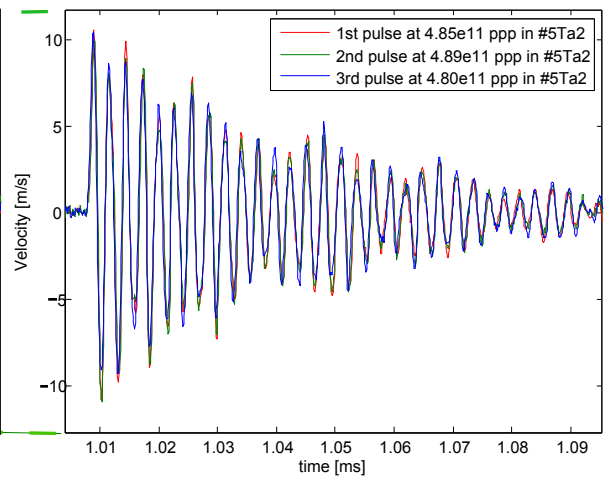

(b)

Figure 5.15: Recorded surface velocity in \#5Ta2 target after being impacted three consecutive times by a beam of $\sim 4.8 \cdot 10^{11} \mathrm{ppp}$. (b) Zoom of the recorded velocity during the first $80 \mu \mathrm{s}$.

Finally, figure 5.15 shows the velocity response of one of the tantalum targets impacted by a $4.95 \cdot 10^{11} \mathrm{ppp}$. Tantalum is without doubt the target that, at least from a dynamic point of view, behaved better during the experiment. It 
can be seen that while the rest of the target materials presented fast a distorted damping of the radial waves when increasing the intensity above $2.2 \cdot 10^{11} \mathrm{ppp}$, tantalum still presented a clean radial wave, even at the highest irradiation intensity as shown in figure 5.16. It is true that certainly the tantalum targets are experiencing plastic deformation, probably even from the first intensity due to its low yield strength. This can be observed in the small damping of the wave shown in figure 5.15-(a) during the consecutively impacted pulses, but in any case, this yielding phenomenon does not seem to have an effect in the radial wave propagation and, differently as happens in the rest of materials, it does not change its frequency. An important point to mention which could have had a contribution is the fact the tantalum targets were the most sensitive to bending modes and bending deformation previously exposed. As it will be described in section 6.3 of Chapter 6, the tantalum \#4Ta1 presented eccentricity deformation at the end of experiment of $4.5 \mathrm{~mm}$, meaning that the interaction between the tantalum target and the proton beam was highly reduced. In any case, the reached surface velocities while conserving the radial wave consistency are high enough (up to $10 \mathrm{~m} / \mathrm{s}$ and $20 \mathrm{~m} / \mathrm{s}$ ) for claiming that, even if the bending effect could have played a role, tantalum behaves much better than the rest of target materials. This better behavior of tantalum in comparison to the other materials is probably the most remarkable finding of the experiment.

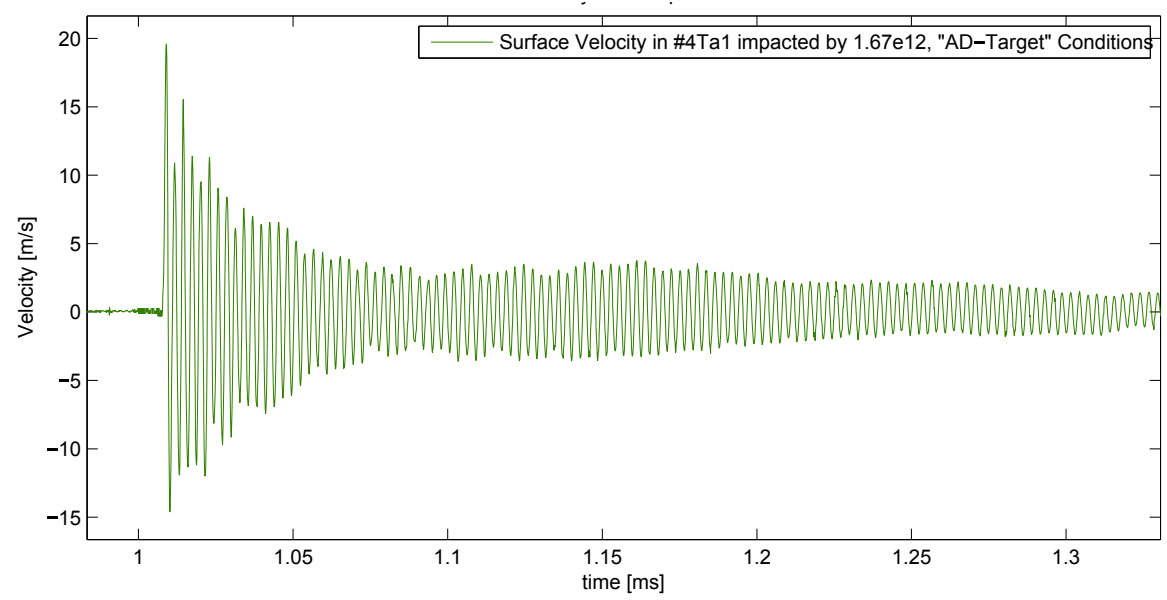

Figure 5.16: Recorded surface velocity in \#5Ta2 target after being impacted by a pulse of $1.67 \cdot 10^{12} \mathrm{ppp}$. It can be seen that the generated radial wave is still clear and undistorted. This is maybe one of the most important finding of the experiment. 


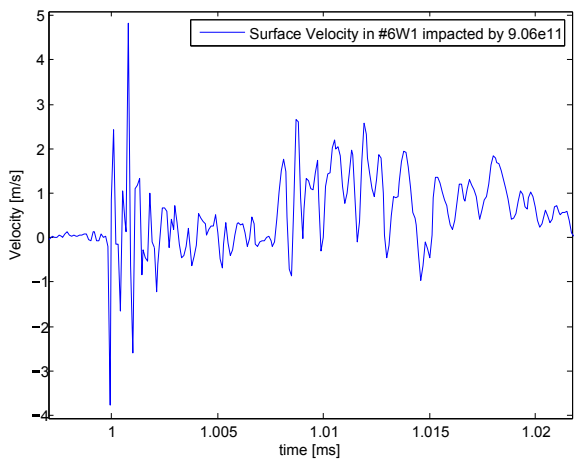

(a)

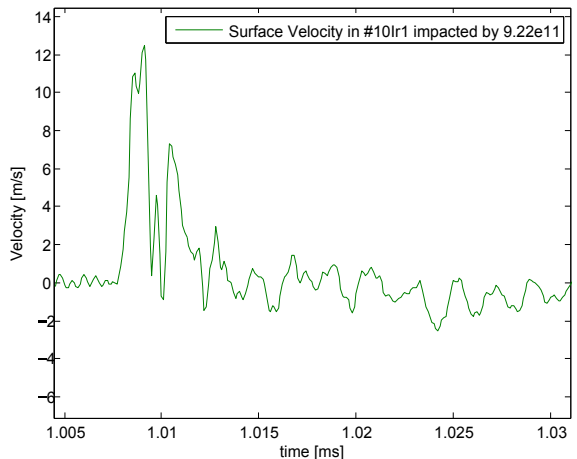

(c)

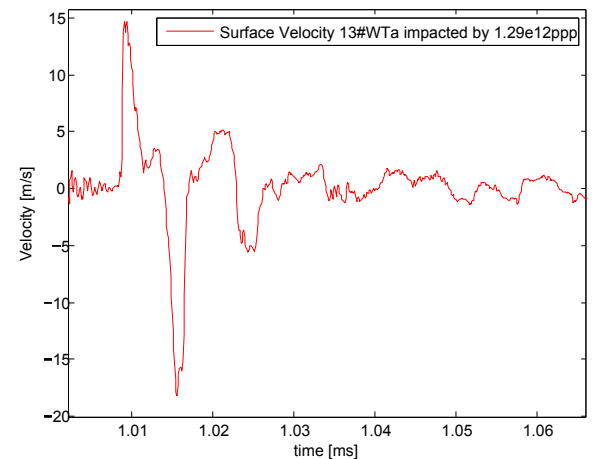

(b)

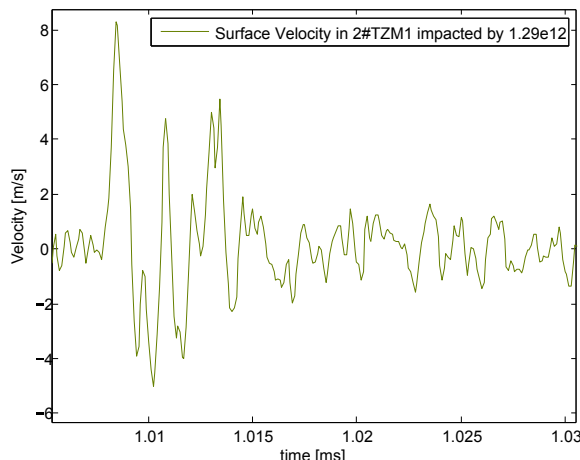

(d)

Figure 5.17: Examples of velocity recorded in tungsten, tungsten cladded in tantalum, iridium and TZM when impacted by intensities above $9.22 \cdot 10^{11} \mathrm{ppp}$. The distorted response of the radial waves measured suggests important internal material damage.

On the other hand, the response of all the targets (except the tantalum ones) when continuing increasing the irradiation intensity is similar to the already distorted response presented previously, with the difference that the damping and distortion of the radial wave is much more accentuated, presenting large changes in frequency, probably due to the interaction of the generated wave with the internal cracks in the material. Figures 5.17-(a)-(d) illustrate this response for tungsten, tantalumcladded tungsten, iridium and TZM respectively. 


\subsection{High Intensity Pulses. Extensive Target Damage}

In this subsection, two special cases are discussed. These cases correspond to the proton beam pulses at the maximum intensity, $1.67 \cdot 10^{12} \mathrm{ppp}$, which were impacted in the two "virgin" targets; \#8W3 and \#12Ir3 as it was shown in table 4.3. The interesting aspect of these cases is that these targets had not been exposed previously to any other impact which would have already produced changes in the material. Therefore, these are the most representative and closest cases to the conditions reached in the AD-Target.

\subsubsection{Tungsten at High Intensity}

Figures 5.18(a)-(b) show the surface velocity and displacement measured by the LDV OptoMET® when impacting a "virgin" tungsten target by a beam of 1.67 . $10^{12}$ ppp. A quite different response in comparison with the previously recorded can be observed. The response is characterized by an abrupt increase of velocity well above $20 \mathrm{~m} / \mathrm{s}$. It was not possible to record the maximum reached velocity due to instrument limitations. No radial wave is easily identified in the velocity plot. Instead, a single oscillation in velocity with a duration of $8 \mu$ s is observed in the figure 5.18(a). This sudden oscillation produces a jump in the displacement of $45 \mu \mathrm{m}$. After this, it is possible to observe the lower frequency vibration produced by the bending mode present in all the impacts.

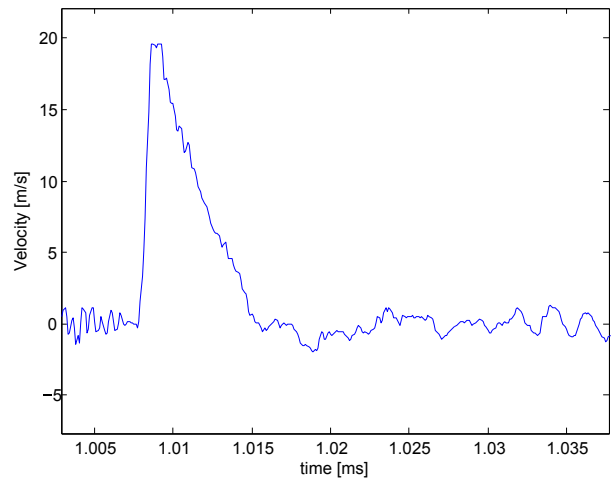

(a)

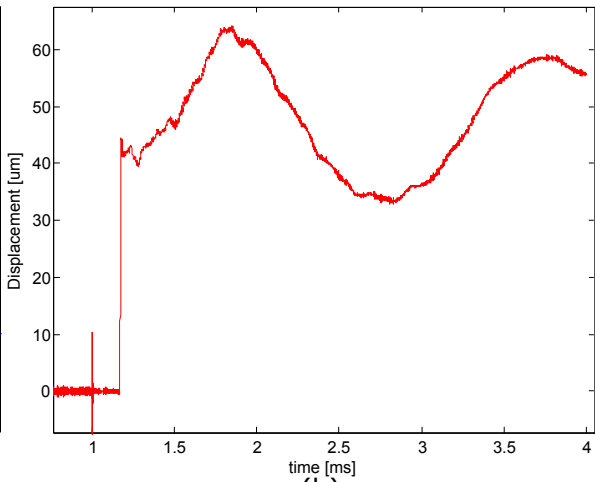

(b)

Figure 5.18: (a) Surface velocity recorded by the LDV OptoMET®during $35 \mu$ s after the first pulse of high intensity $\left(1.67 \cdot 10^{12} \mathrm{ppp}\right)$ impacted in the "virgin" \#8W3 target. (b) Surface displacement corresponding to the impact.

Figure 5.19 shows the temperature measured by the thermocouples in this target, not only during this first pulse, but also during eight other pulses of the same intensity which were consecutively impacted. The maximum temperature recorded 
on the target surface after the first pulse was $500{ }^{\circ} \mathrm{C}$. It is very interesting to observe how the maximum reached temperature progressively decreased even if the impacted intensity was the same in the following impact series. This decrease of the maximum temperature is explained by the reduction of target-beam interaction produced by the permanent bending deformation of the target, quantified after the experiment in a maximum eccentricity of $4.8 \mathrm{~mm}$ with respect to the initial target axis as it is shown in 6.1 of Chapter 6.

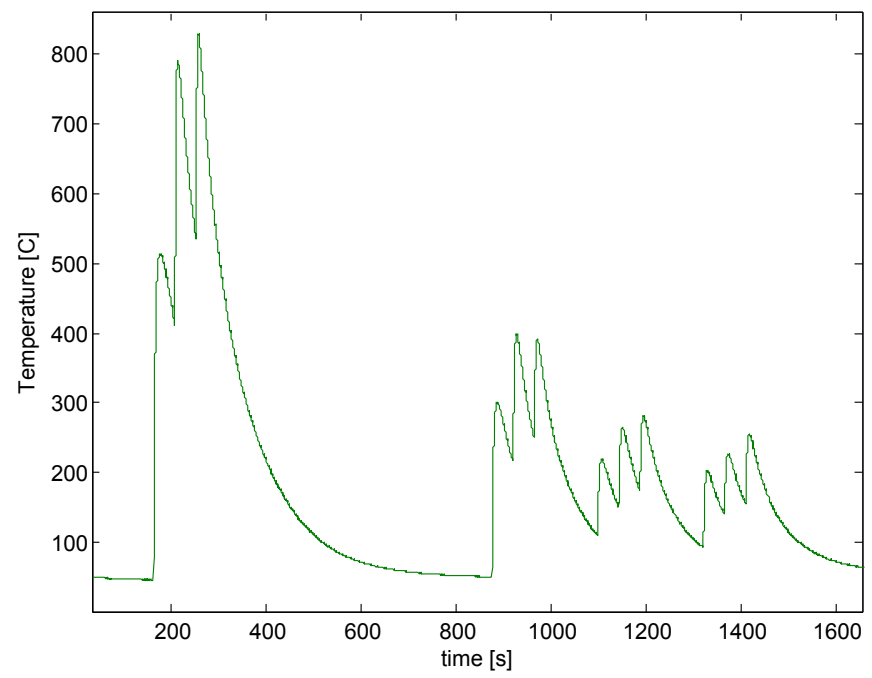

Figure 5.19: Temperature measured by one of the surviving thermocouples attached to the \#8W3 when impacted by twelve consequtive pulses of high intensity. The clear decrease of the maximum temperature reached is explained by the progressive bending of the target and reduction of beam-target interaction as discussed in section 6.3.

\subsubsection{Massive Fragmentation of the Iridium Target}

In the same way as done with the \#8W3 target, the \#12Ir3 iridium target was left "virgin" during experiment and impacted at the end by a pulse of $1.71 \cdot 10^{12} \mathrm{ppp}$. This impact produced an impressive response very different from the impacts in all the other targets. As can be seen in the picture of figure 5.20, the target was massively fragmented following a bulb-shape which is consistent with the longitudinal profile of deposited energy in the target shown in figure 4.5 of Chapter 4 . The cause of this mode of failure is probably the sudden and extensive internal cracking during the pulse impact, provoking that the inertia of the expansion rapidly propagated these cracks towards the target surface, resulting in the observed target splitting in longitudinal filaments. This filament splitting could be also related to the manufacturing method of the target, which was swaged after forging, leading 
to grain deformation on the longitudinal direction. Another reason could be that a change of phase (melting) took place in the inner part of the target, considering that the impacted intensity was slightly higher than initially foreseen and that iridium has a melting point of only $2450{ }^{\circ} \mathrm{C}$. This sudden change of phase could have created a shock wave which traveled towards the surface. However, this last option is excluded by recent investigations, such as more accurate simulations with the real beam parameters of this impact, which showed that the maximum temperature reached was only $2000{ }^{\circ} \mathrm{C}$, as well as post irradiation examinations of the core material by microscope, which did not show any track of material melting in the micro-structure. Even if the conditions reached on the target surface were extreme during the pulse impact, the online instrumentation still recorded data.

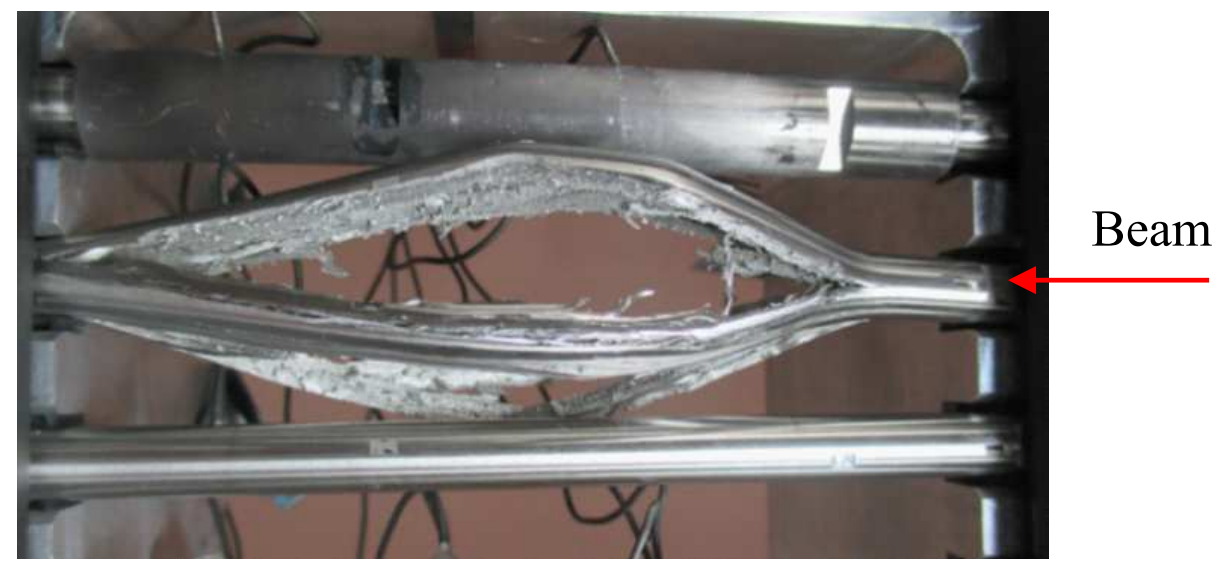

Figure 5.20: Picture of the state of the \#12Ir3 target after a single beam impact of $1.71 \cdot 10^{12} \mathrm{ppp}$.

Figures 5.21(a)-(c) show the velocity and displacement reached on the target surface. It can be seen that the LDV saturated as the surface velocity exceeded $20 \mathrm{~m} / \mathrm{s}$. In addition, it is very interesting to observe in figure 5.21-(b) that, during the first instants, this velocity response presents three oscillations after the pulse impact. These oscillations have initial periods of $0.9 \mu \mathrm{s}$, less than half of the period of radial wave in iridium which was measured in $2.3 \mu \mathrm{s}$, and could indicate that the fast cracking is progressively taking place when the material is subjected to the tensile part of the dynamic response, up to the point in which it can no longer sustain tensile stresses. Figure 5.21-(c) shows the surface displacement at the point of measurement obtained by the integration of the velocity. A deformation of $1.9 \mathrm{~mm}$ was recorded in less than $1 \mathrm{~ms}$. This displacement was probably even larger, considering that the recorded velocity was saturated. 


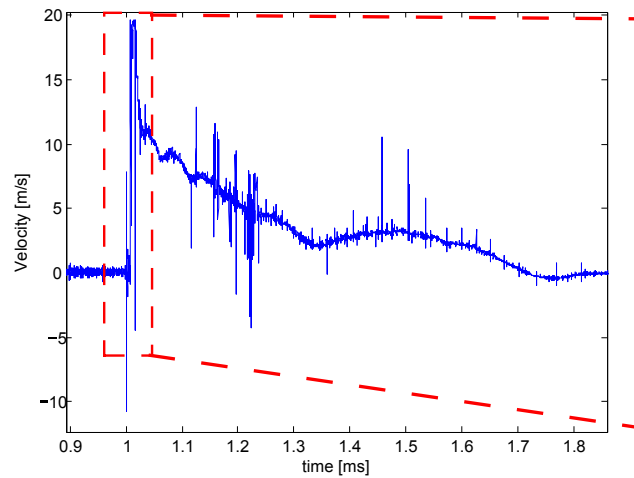

(a)

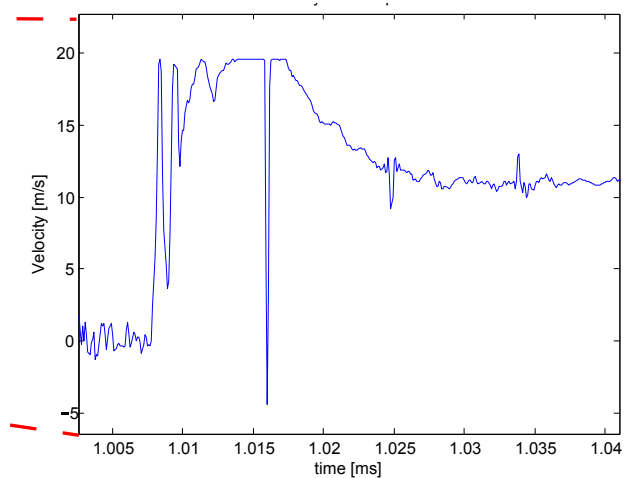

(b)

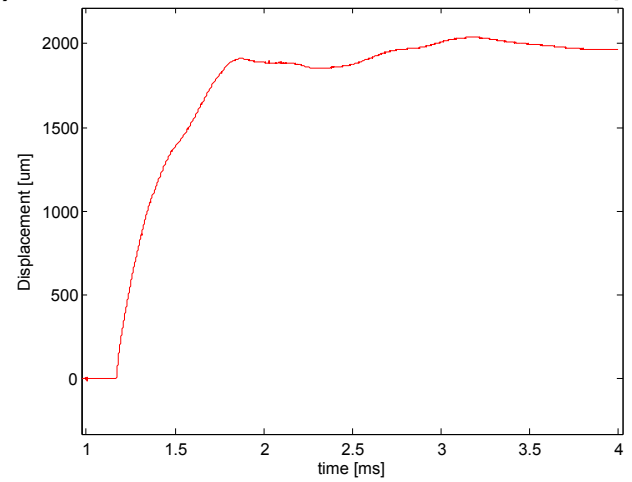

(c)

Figure 5.21: Response measured by the LDV during the impact which caused the massive fragmentation of the iridium \#12Ir3 target. (a) Velocity recorded during $800 \mu \mathrm{s}$. (b) Zoom of the during the first $30 \mu \mathrm{s}$. (c) Recorded displacement.

Figure 5.22(a) shows the temperature recorded by the pyrometer during the target fragmentation. It is interesting to observe that an extremely fast temperature oscillation was recorded at the target surface, within a time scale of $125 \mu \mathrm{s}$. The instrument saturated at its maximum range of acquisition, which was $1400^{\circ} \mathrm{C}$. An eighth-order-polynomial data fitting is presented in figure 5.22-(b), which suggests that the measured temperature could have reached up to $1500{ }^{\circ} \mathrm{C}$. After this peak, the measured temperature drops down to $700^{\circ}$ in $70 \mu \mathrm{s}$. It is certainly hard to explain this huge and fast drop of $800{ }^{\circ} \mathrm{C}$ by heat transfer processes. A possible explanation could be the displacement of the mirror where the pyrometer was pointing due to the target fragmentation, with the corresponding change of emissivity since this mirror was specifically painted in black. 


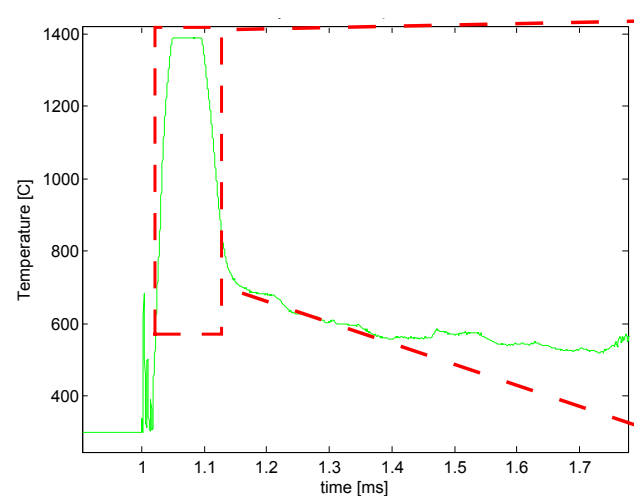

(a)

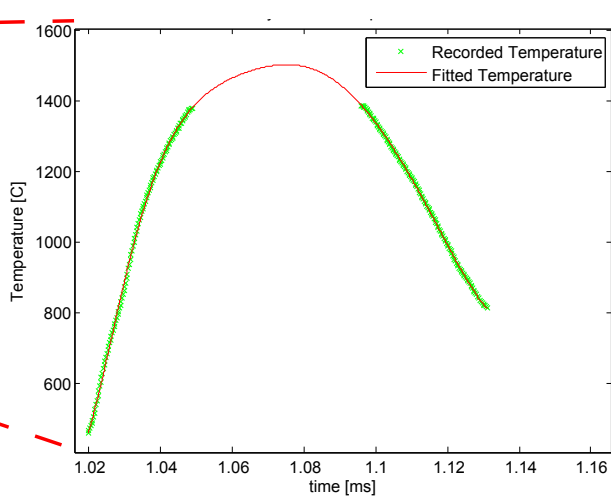

(b)

Figure 5.22: (a) Temperature recorded by the pyrometer during the impact which caused the massive fragmentation of the iridium \#12Ir3 target. (b) Data extrapolation of the temperature recorded during the peak taking place in the first $70 \mu$ s after the impact. 


\subsection{Summary of Response of the Irradiated Targets}

In this section a summary of the results obtained for all the irradiated materials is presented. This summary is carried out by showing specific quantitative information extracted from each single impact and plotting it as a function of the impacted intensity. Figure 5.23 shows the maximum surface velocity recorded by LDV OptoMETR for each single pulse. Normally, this maximum velocity is reached during the first radial oscillation after the pulse impact, therefore it is a good indicator of the state of the material and the level of internal damage suggested by the distorted and damped radial response discussed in section 5.3.

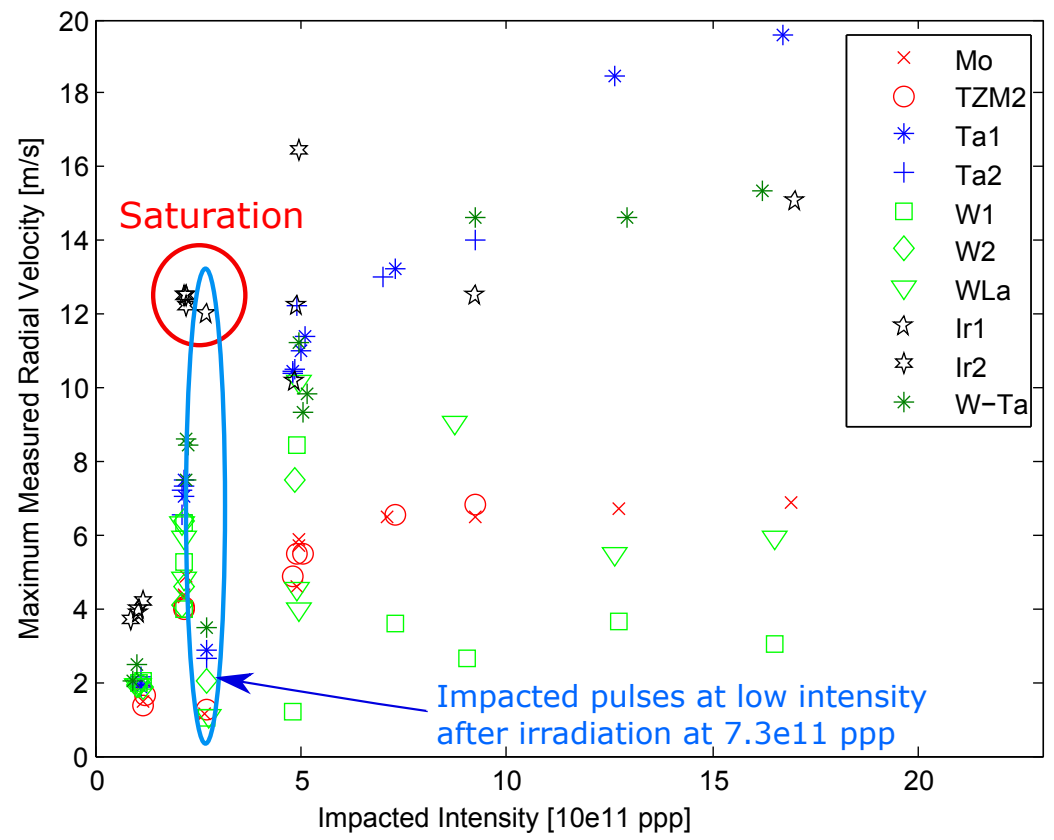

Figure 5.23: Summary of maximum recorded surface velocity during the experiment for the irradiated materials.

Important differences in the response of the materials can be observed. For example, it is certainly clear that tungsten (light green) was experiencing important damage from the $2^{\text {nd }}$ irradiation intensity, $2.17 \cdot 10^{11} \mathrm{ppp}$. It can be observed that during the subsequent impacted pulses at this intensity the tungsten targets presented low external velocities. In addition, in the next pulses at higher intensities, the maximum velocity reached was similar or even decreasing. Lanthanumdoped tungsten target (WLa) seems to have a slightly better behavior since the reached velocities are higher, but in any case it is possible to observe a drop in the 
maximum reached velocity when increasing intensity. On the other hand, the Tacladded tungsten target seems to present a better response, showing a monotonic increase of maximum velocities up to $5 \cdot 10^{11} \mathrm{ppp}$. Molybdenum and TZM targets show a similar response, reaching always almost the same maximum velocity when increasing the intensity above $5 \cdot 10^{11} \mathrm{ppp}$. The iridium targets presented very high velocities during the first pulses, causing the saturation of the instrument when these velocities were above $12.5 \mathrm{~m} / \mathrm{s}$. However, after irradiation at $5 \cdot 10^{11} \mathrm{ppp}$ of the iridium targets, it is possible to observe a large drop of the maximum reached velocity. Nevertheless, it is interesting to observe that iridium presented a better response than tungsten even if its density is $20 \%$ more. In any case, it is believed that all these targets present internal damage from the first or second irradiation sequence as suggested by the changes in the measured radial wave. Tantalum targets are the only ones which external reached velocities continued monotonically growing during the whole experiment, reinforcing what was observed looking at its "clean" a and non-distorted dynamic response, which was present even at the highest intensity. It is interesting to note as well in the figure 5.23 the effect of permanent damage and plastic hardening by noticing the significant lower velocities reached in all the materials at the second irradiation of $2.7 \cdot 10^{11} \mathrm{ppp}$, performed after exposing them already to $7.3 \cdot 10^{11} \mathrm{ppp}$.

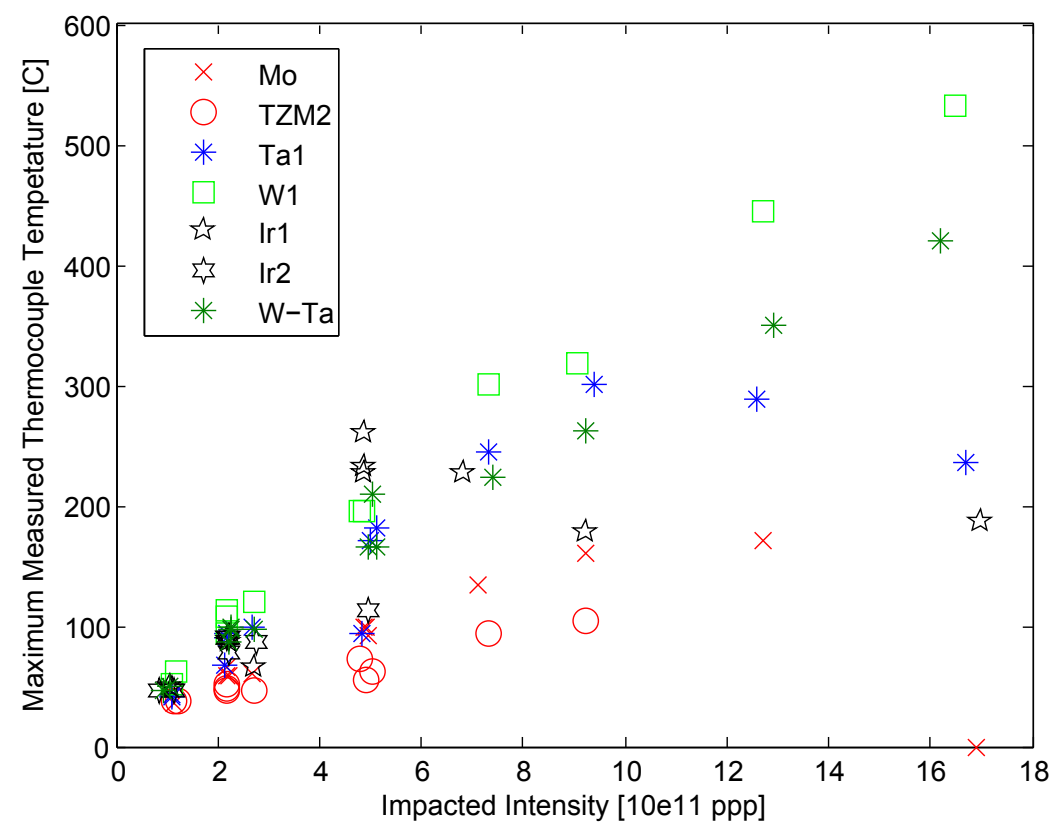

Figure 5.24: Summary of maximum recorded temperature by the thermocouples during the experiment. 
In a similar way as figure 5.23, figure 5.24 shows the maximum temperature measured by the thermocouples, which were attached to the target surface, as a function of the impacted intensity. The plot shows only the thermocouples which survived the inertia forces and continued attached until the end of the experiment so their measurements can be considered reliable. It is interesting to observe a monotonically increase of the temperature with the intensity for all the targets except tantalum and iridium. However, this monotonic increase is far from being directly proportional, even in the targets that did not present large levels of bending deformation at the end of the experiment (see table 6.1). This fact could suggest a reduction of beam-target interaction due internal cracking in the targets. In addition, it can be observed a very clear drop in maximum temperatures reached in \#10Ir1 and \#5Ta1 targets from $5 \cdot 10^{11} \mathrm{ppp}$ and $9 \cdot 10^{11}$ ppp respectively. This reduction is a direct consequence of the decrease of beam-target interaction due to the large and progressive bending deformation that these targets were presenting. As it will be discussed in section 6.3, the tantalum and iridium targets were presenting maximum eccentricities of more than $4 \mathrm{~mm}$ at the end of the experiment, meaning that, in their central regions, they were only reached by the beam halo. This could explain why the target \#10Ir1 did not present a massive fragmentation like the "virgin" iridium target (\#12Ir3) even if a high intensity beam was sent as well to the former. The reduction of beam-target interaction taking place in the tantalum target due to bending deformation could have had as well a contribution to the better dynamic response observed in this target. However, figure 5.24 demonstrates that this could not have been the only reason, as the maximum recorded velocity in tantalum was always monotonically raising.

At this point, it is important to clarify an apparent incoherence in the response of target \#5Ta1 in the figures 5.23 and 5.24. As already mentioned, the recorded velocity of this target is monotonically increasing with the intensity in the former, while the temperature is decreasing in the latter. This decrease is attributed to the reduction of beam-target interaction due to progressive bending of the target. One may claim that this decrease of interaction should have produced also a reduction in the maximum recorded velocity at high intensity. This, however, is not necessarily true if one takes into account the position at which velocity was measured, which it was placed close to the upstream support of the target. In this location, the bending deformation is much less accentuated and therefore not influencing that much the energy deposited there by the beam, leading to the high velocities recorded and presented in figure 5.23. 


\subsection{Discussion}

This chapter presented a detailed analysis of the most significant online results recorded during the 139 high intensity, $440 \mathrm{GeV}$, proton pulses impacted onto 13 targets of Ir, Mo, TZM, Ta, W, W-La and W cladded in Ta. A large amount of online data of the targets surface velocity, displacement and temperatures was measured. The radial and longitudinal waves (or modes) whose periods were analytically predicted in Chapter 2 as well as by hydrocode simulations in Chapter 3 were recorded, in particular at low intensity pulses. A third lower frequency wave was also recorded and identified as an excited bending mode resulting from minor off-axis deviation of the beam impacts. In addition, it was possible to infer progressive alteration and damage in the target materials while increasing the impacted intensity by changes in the surface radial velocity of the targets recorded online.

The main outcome of the experiment is that most of the irradiated targets (all except tantalum) were suffering significant internal damage even from reached conditions 5-7 times lower than the ones taking place in the real AD-Target, indicating that the iridium core of such target may be seriously damaged and fragmented from the very beginning of operation. The predicted radial compressive-to-tensile wave studied in detail in Chapter 3 is confirmed to be the main damaging phenomena and probably the cause of the drop in antiproton production often observed during the first days of operation, since fragmentation of the target core may lead to loss of its effective density and primary beam/target interaction.

The excellent response of tantalum in the experiment (which showed a non-distorted radial wave even at the highest intensity pulses, and therefore indicating absence of internal cracks probably due to energy release processes through plastic yielding rather than crack formation) makes it the strongest candidate material for the future AD-Target design. Furthermore, the identification of the radial compressiveto-tensile wave as main short-term damaging phenomenon motivates the pursuit of strategies to mitigate the effect of this wave in the new design, such as relaxation of beam primary optics or changes in the target core geometry.

In the next chapter, first post irradiation examinations such as measurements of the targets deformation, pictures, and ultrasound inspections will complement the online results just presented. In addition, experimental vs simulations cross checks are included, assessing the validity of the used hydrocodes. 


\section{Chapter 6}

\section{The HRMT27 Experiment: First PIEs and Experiment vs Simulation Comparisons}

In Chapters 4 and 5 a detailed description of the HRMT27-RodTarg experiment and measured online results were respectively presented. The present chapter completes these two with the first PIEs (Post Irradiation Examinations) performed after the vacuum tank opening and targets dismounting. Pictures with the state of the targets after irradiation as well as measurements of their bending deformation done by CMM (coordinate measuring machine) are shown. In addition, simulations vs experimental data comparisons are presented for $\mathrm{Ta}, \mathrm{W}$ and $\mathrm{Ir}$ at different regimes, from low intensity pulses (in which internal fracture was not taking place yet) to middle and high intensities at which internal failure was occurring. Benchmarking of the minimum hydrostatic pressure model for $\mathrm{W}$ and Ir using the experimental data recorded is included in section 6.4.2. Finally, a summary discussion of main achievements of the experiment and proposed improvements is presented in section 6.6. 


\subsection{Pictures of the Targets after the Experiment}

After 3 months of radioactive cooling, the residual dose of the irradiate targets was low enough to allow tank opening. After 6 months the targets were dismounted from the sample holder and photographed individually. At this time the residual doses at contact of the targets were from 30 to $80 \mu \mathrm{Sv} / \mathrm{h}$, depending on the material and the corresponding accumulated protons on target (POT). In the next subsections these pictures are shown and discussed.

\subsubsection{Mo and TZM targets}

Figure 6.1(a)-(c) shows the post irradiated Mo and two TZM targets respectively. As it can be seen in the picture, a significant change of the color in the surface took place, attributed to surface oxidation. It must be mentioned that this color change did not happen during irradiation but after water immersion of the targets during ultrasound test (UT) measurements. Therefore it was not caused by the proton irradiation.
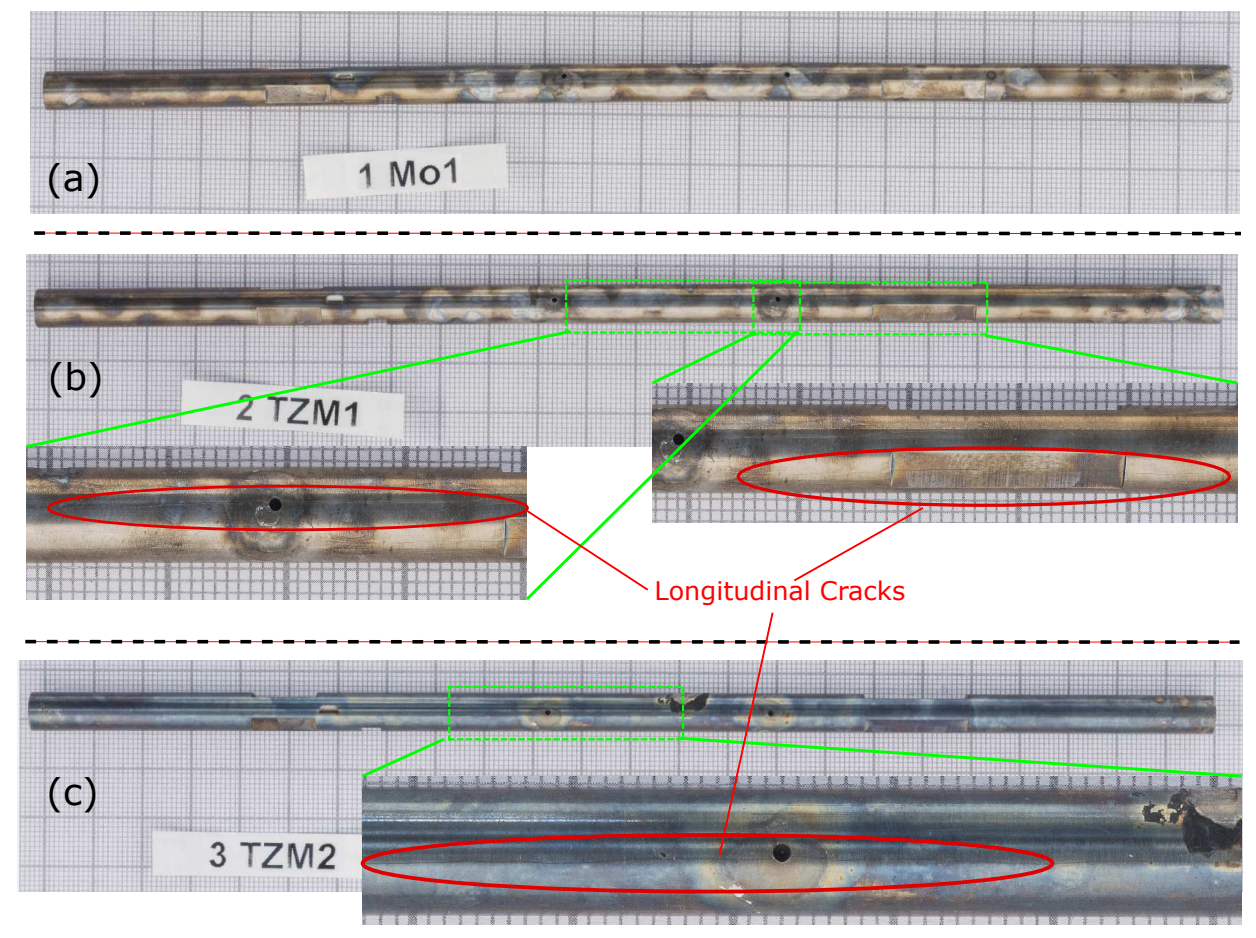

Figure 6.1: Pictures of Mo and TZM targets after the experiment. Several longitudinal cracks are perceptible along the targets 
In the pictures it is possible to observe that the three targets show a perceptible level of bending. Quantification and causes of this bending are analyzed in detail in the next section of the present chapter (section 6.3). \#1Mo1 target received a total of $7.3 \cdot 10^{12}$ POTs, and \#2TZM1 and \#3TZM2 of $8.4 \cdot 10^{12}$ and $4.3 \cdot 10^{12}$ respectively. \#1Mo1 and \#2TZM1 targets were irradiated up to the maximum intensity while \#3TZM2 was only irradiated up to intermediate intensities $\left(\sim 9.1 \cdot 10^{11} \mathrm{ppp}\right)$.

Several longitudinal cracks can be observed in the pictures, even in \#3TZM2 target which did not see the most extreme conditions. Internal cracks were also detected by ultrasound inspections. This is consistent with the hypothesis extracted by online measurements presented in section 5.3 of Chapter 5 , in which it was deduced that Mo and TZM targets were suffering internal damage from impacted intensities of $2.1 \cdot 10^{11}$ to $5 \cdot 10^{11} \mathrm{ppp}$.

\subsubsection{Ta targets}

Figures 6.2 and 6.3 show pictures of the \#4Ta1 and \#5Ta2 targets respectively. These targets were irradiated by a total of $7.2 \cdot 10^{12}$ and $4.2 \cdot 10^{12}$ POTs. \#4Ta1 was irradiated up to the maximum intensity, $1.6 \cdot 10^{12} \mathrm{ppp}$, while \#5Ta2 only up to an intermediate intensity of $\sim 9.2 \cdot 10^{11} \mathrm{ppp}$.

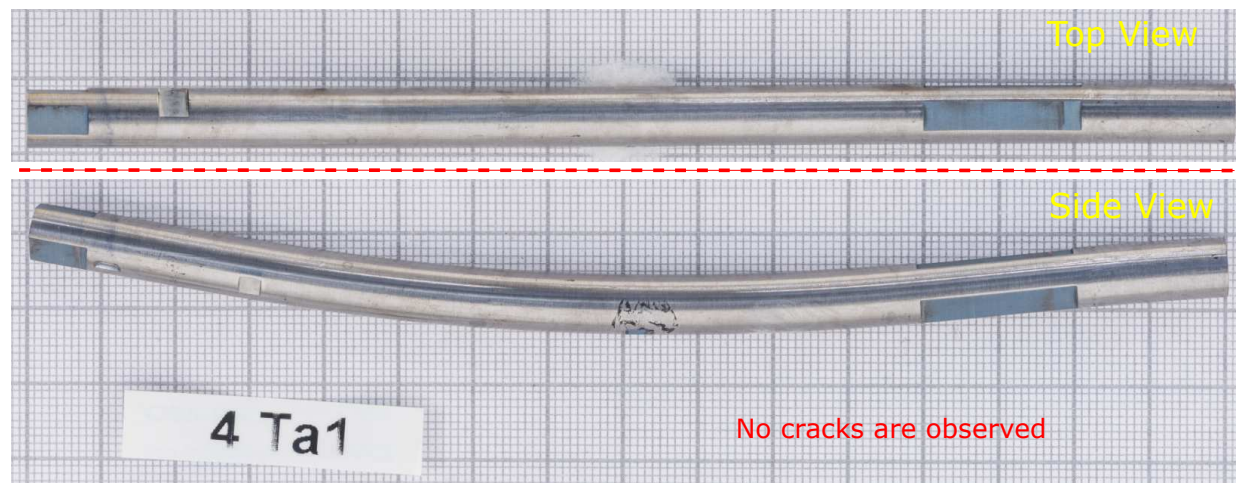

Figure 6.2: Pictures of \#4Ta1 targets in two different views. This target was exposed to AD-Target conditions without presenting internal cracking apparently, demonstrating the superior response of $\mathrm{Ta}$ in comparison to the rest of irradiated materials. The large level of bending in discussed in section 6.3.

As it can be seen in the pictures, both targets present a very large level of bending (quantified in table 6.1 of section 6.3). The bending is more accentuated in target \#4Ta1 since it saw high intensity beams. Apart from bending, it is very interesting to mention that no cracks were observed in any of these targets, neither in the surface nor internally by ultrasound inspections. Therefore, it seems that tantalum 
is the only material that survived experiment without presenting internal cracking. This result is consistent with the "clean" and not distorted radial wave which was was measured online even at highest intensity in section 5.3 of Chapter 5 . Future foreseen PIEs consisting in slicing the targets and observing their microstructure by means of optical microscope and SEM will be carried out to confirm this hypothesis and to study the changes in the material due to extensive yielding.

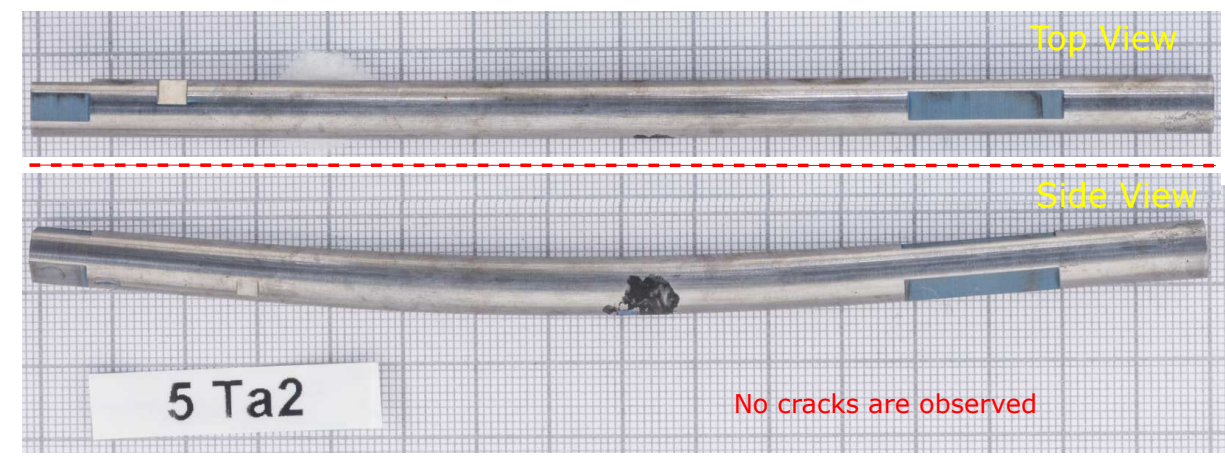

Figure 6.3: Pictures of \#5Ta2 target in two different views. This target was irradiated up to $9.2 \cdot 10^{11} \mathrm{ppp}$ (60\% of the AD-Target conditions). The black spot observed in the target is the high emissivity paint required for the pyrometer measurements.

\subsubsection{W and W-La targets}

Figures $6.4,6.5,6.6$ and 6.7 show pictures of the \#6W1, \#7W2, \#8W3 and \#9WLa targets respectively. These targets were irradiated by a total of $6.4 \cdot 10^{12}$, $1.6 \cdot 10^{12}, 2 \cdot 10^{13}$ and $7 \cdot 10^{12}$ POTs. \#6W1 and \#9WLa were irradiated up to the maximum intensity $\left(1.6 \cdot 10^{12} \mathrm{ppp}\right)$ while \#7W2 only up to an intermediate intensity of $4.8 \cdot 10^{11}$ ppp. \#8W3 target was only irradiated with the highest intensity pulses, receiving up to 12 consecutive pulses of $\sim 1.6 \cdot 10^{12} \mathrm{ppp}$.

In figure 6.4 it can be observed the large extend of cracks of the \#6W1 target. These cracks are mostly longitudinal but also transverse cracks are present, the latter initiated close to one of the thermocouple holes, which was acting as stress concentrator. Spall of a fragment close to the transverse crack can be also observed, caused by the inertia and high tensile wave reached close to the surface. The high brittleness of tungsten is clearly revealed by the state of the targets after the experiment, which are apparently the most cracked even when compared with the other irradiated materials also known to be brittle. Internal cracking of tungsten was also shown by ultrasound tests.

In figure 6.5 it can be observed the \#6W2 target after irradiation. This target, even if only exposed $5 \cdot 10^{11}$ ppp (3 times less than AD-Target conditions), presents also a large extend of longitudinal cracks. In the same way as target \#6W2, it 


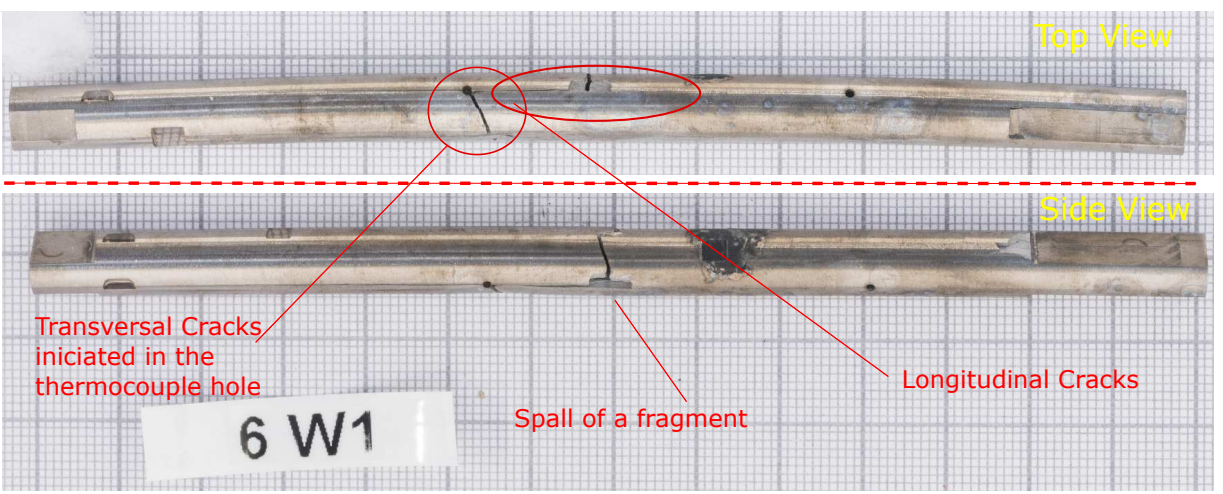

Figure 6.4: Pictures of \#6W1 target in two different views. This target was irradiated up to $1.6 \cdot 10^{12} \mathrm{ppp}$. A large extend of cracking and even spalling can be observed. The black spot observed in the target is the high emissivity paint required for the pyrometer measurements.

also presents transverse cracks initiated close to one of thermocouple holes. The cracks observed were also detected by ultrasound inspections. This high level of cracking even from middle and low intensity is consistent with what it was observed in the online measurements presented in section 5.3 of Chapter 5 , in which it was deduced that tungsten targets were suffering internal damage from intensities from $2.1 \cdot 10^{11} \mathrm{ppp}$.

In figure 6.6 is shown the \#8W3 target after irradiation. This target received up to 12 consecutive pulses of $\sim 1.6 \cdot 10^{12} \mathrm{ppp}$ (similar as AD-Target conditions). As expected, a large number of longitudinal cracks are present. In addition, the target was extensively bended (discussed more in detail in section 6.3). This target is the tungsten one that better represents the potential response of this material at AD-Target conditions.

Finally, figure 6.7 shows a picture of the \#9WLa target, made of tungsten doped with lanthanum oxide. This target was irradiated from low intensity up to the maximum intensity $1.6 \cdot 10^{12} \mathrm{ppp}$. As can be seen in the picture, and also observed by ultrasound tests, the target is also considerably cracked. Longitudinal and transverse cracks are observed. This is consistent with the fact, already mentioned in section 1.5.3 of the introduction, that tungsten doped with lanthanum does not necessarily present an improved behavior in terms of brittleness in comparison to pure tungsten. In this context, it is hard to notice at this stage (from the pictures and online data recorded) any significant improvement of the response of this target with respect to the pure tungsten one shown in figure 6.5. 


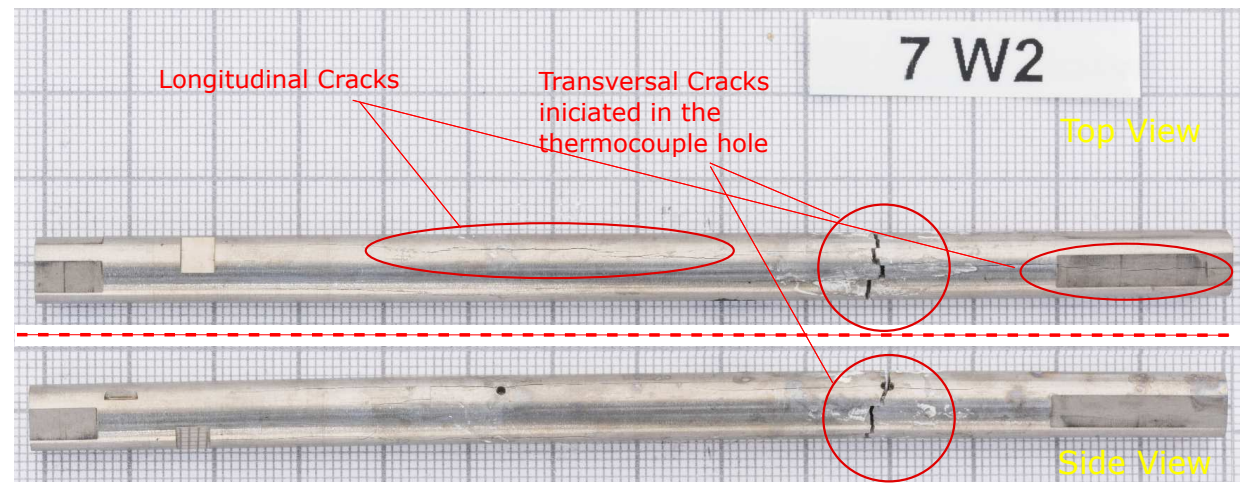

Figure 6.5: Picture of \#7W2 target in two different views. This target was irradiated up to $5 \cdot 10^{11} \mathrm{ppp}$ (3 times less than AD-Target conditions). A large extend of cracking is also observed for this target

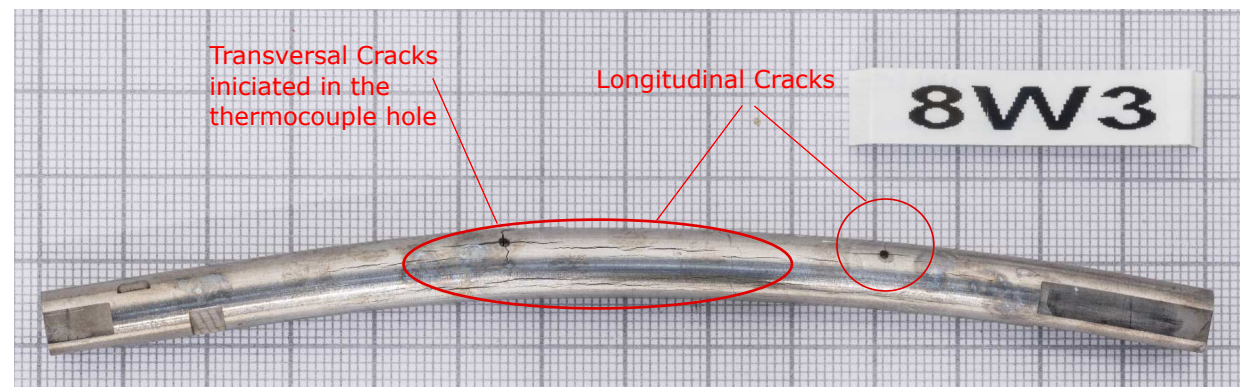

Figure 6.6: Picture of \#8W3 target, irradiated by 12 consecutive high intensity pulses of $1.6 \cdot 10^{12} \mathrm{ppp}$

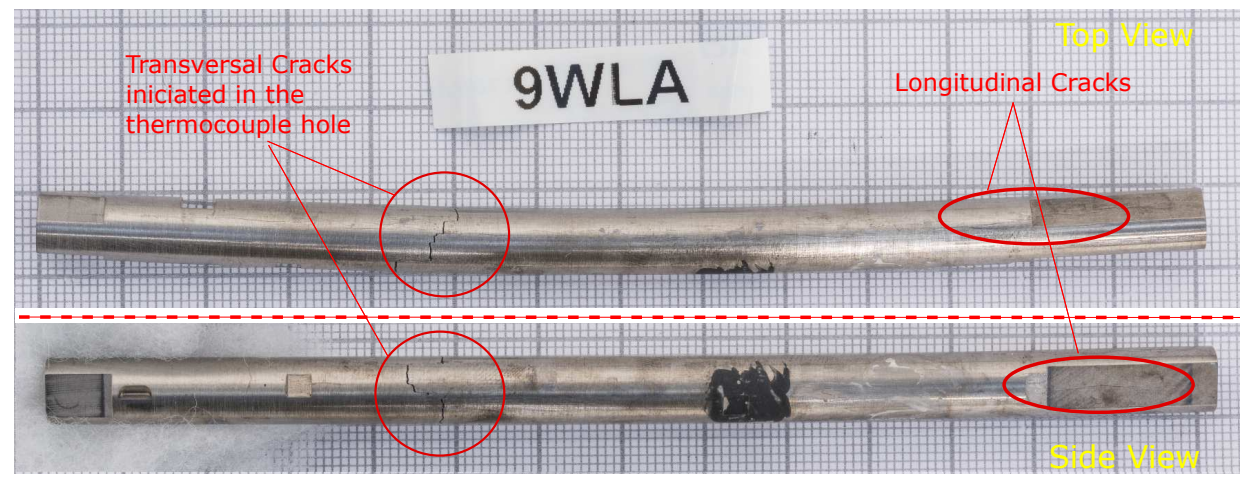

Figure 6.7: Picture of \#9WLa target in two different views. This target was irradiated up to $1.6 \cdot 10^{12} \mathrm{ppp}$. 


\subsubsection{Ir targets}

Figures 6.8, 6.9 and 6.10 show pictures of the \#10Ir1, \#11Ir2 and \#12Ir3 targets respectively. These targets were irradiated by a total of $7.11 \cdot 10^{12}, 1.6 \cdot 10^{12}$, and $1.71 \cdot 10^{12}$ POTs, with the latter (\#12Ir3) receiving only a single high intensity pulse. \#10Ir1, was irradiated up to the maximum intensity $\left(1.6 \cdot 10^{12} \mathrm{ppp}\right)$ while \#11Ir2 only up to an intermediate intensity of $4.8 \cdot 10^{11} \mathrm{ppp}$.

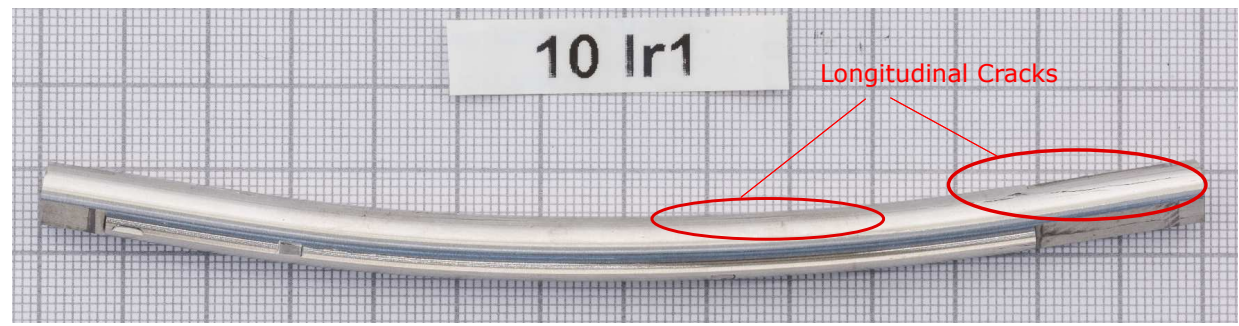

Figure 6.8: Picture of \#10Ir1 target irradiated up to $1.6 \cdot 10^{12} \mathrm{ppp}$. Several longitudinal cracks as well as a large level of bending of the target took place.

It is possible to observe in figure 6.8 several longitudinal cracks in the \#10Ir1 target, irradiated up to the maximum intensity. In addition, this target presented a large level of bending deformation (discussed in section 6.3). However, in comparison to the tungsten targets, no traverse cracks have been observed in the surface. Furthermore, the comparison of the amount of longitudinal cracks and the level of bending suggests that iridium presents, at first sight, a more ductile response than tungsten.

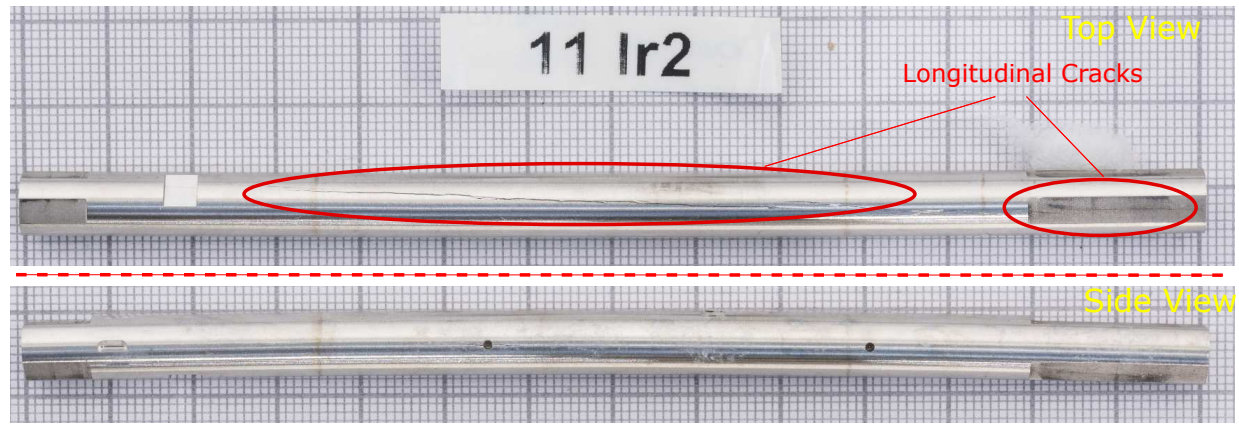

Figure 6.9: Picture of \#11Ir2 target in two different views. This target was irradiated up to $4.8 \cdot 10^{11} \operatorname{ppp}(3$ times less than $\mathrm{AD}$-Target conditions).

Figure 6.9 presents the \#11Ir2 after irradiation up to an intermediate intensity of $4.8 \cdot 10^{11} \mathrm{ppp}$. In the same way as TZM and tungsten targets, the presence of longitudinal cracks already at this stage supports the online measurements presented in section 5.3 of Chapter 5 . 
Finally, figure 6.10 presents state the target \#12Ir3, already introduced in section 5.4.2. This target massively fragmented after the impact of a single pulse of of $1.71 \cdot 10^{12} \mathrm{ppp}$ (AD-Target conditions) ${ }^{1}$. The fragmentation of the target took place following a bulb-shape which is consistent with the longitudinal profile of deposited energy in the target. The reason why this target presented such type of fragmentation while the target \#10Ir2 (which apparently saw the same intensity) did not, is that the level of bending of the latter, caused by the successive previous pulses, made that the high intensity beam barely interacted with this target, as it will be shown in section 6.3 .

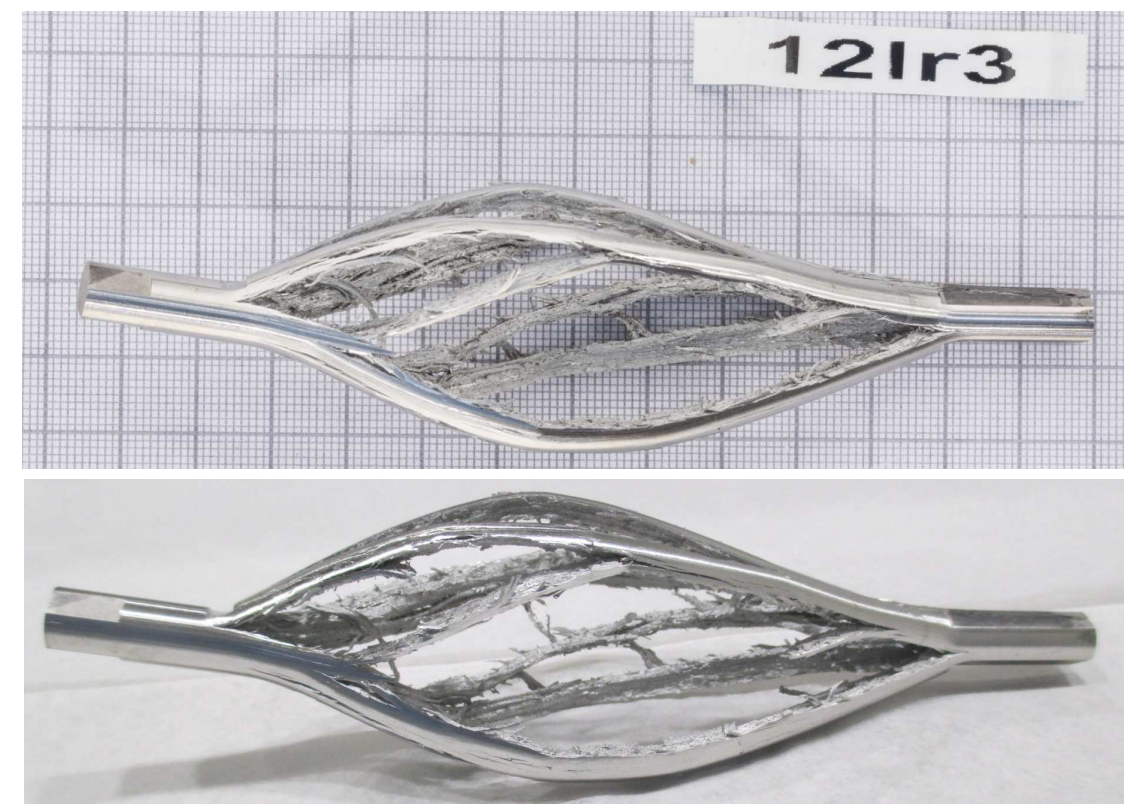

Figure 6.10: Pictures of the fragmentation of target \#12Ir3 after a single impact of a proton beam of $1.71 \cdot 10^{12} \mathrm{ppp}$ with a size of $1.82 \times 1.65 \mathrm{~mm}$ at $1 \sigma$.

The fracture shape consisting of filament splitting shown in figure 6.9 could be consistent also with the manufacturing method of the target, which was swaged after forging, leading to grain deformation in the longitudinal direction. Simulation showed in figure 6.11 , considering the exact beam intensity $\left(1.71 \cdot 10^{12} \mathrm{ppp}\right)$ and size $(1.82 \times 1.65 \mathrm{~mm}$ at $1 \sigma)$ of this pulse estimates that the maximum temperature reached was $2016{ }^{\circ} \mathrm{C}$. Thus, rejecting the hypothesis that this sort of fracture

\footnotetext{
${ }^{1}$ It is true that this intensity is slightly higher than the initially foreseen as "AD-Targe conditions", which was $1.5 \cdot 10^{12}$ ppp. This higher intensity was requested during the experiment in order to compensate for the observed beam sizes which were also higher than the ones initially foreseen due to beam-line optics $(1.82 \times 1.65 \mathrm{~mm}$ at $1 \sigma$ instead of $1.5 \times 1.5 \mathrm{~mm})$. With this correction, the maximum temperature reached was in the order of $2000{ }^{\circ} \mathrm{C}$ as shown by figure 6.11 , equivalent to the beam parameters initially foreseen.
} 
was initiated by internal melting of the target. Absence of a melted phase was as also confirmed by microscope examinations, which did not show any track of melting material in the micro-structure (such as spherical particles generated after an eventual fast re-solidification)

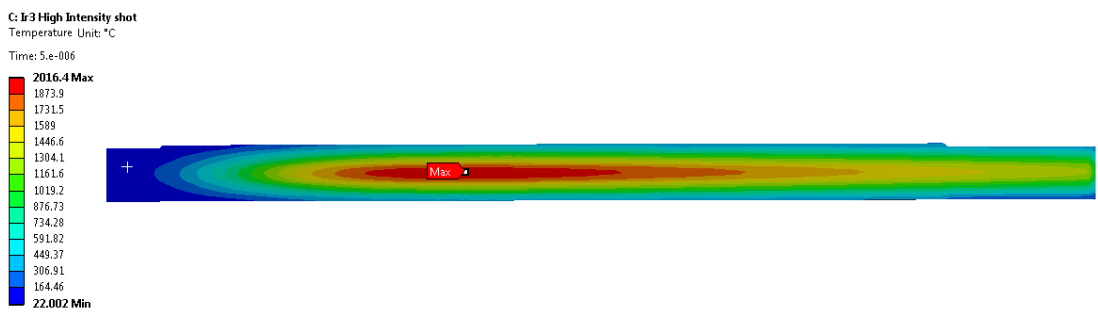

Figure 6.11: Simulated temperature profile of the \#12Ir3 target produced as a consequence of the impact of the proton beam of $1.71 \cdot 10^{12} \mathrm{ppp}$ intensity and size of $1.82 \times 1.65 \mathrm{~mm}$ at $1 \sigma$, which caused the massive fragmentation shown in figure 6.10 .

\subsubsection{W-Ta cladded target}

Figure 6.12 shows a picture of the tungsten target cladded in tantalum. This target received a total of $7.22 \cdot 10^{12}$ POTs and it was irradiated up to $1.62 \cdot 10^{12} \mathrm{ppp}$.

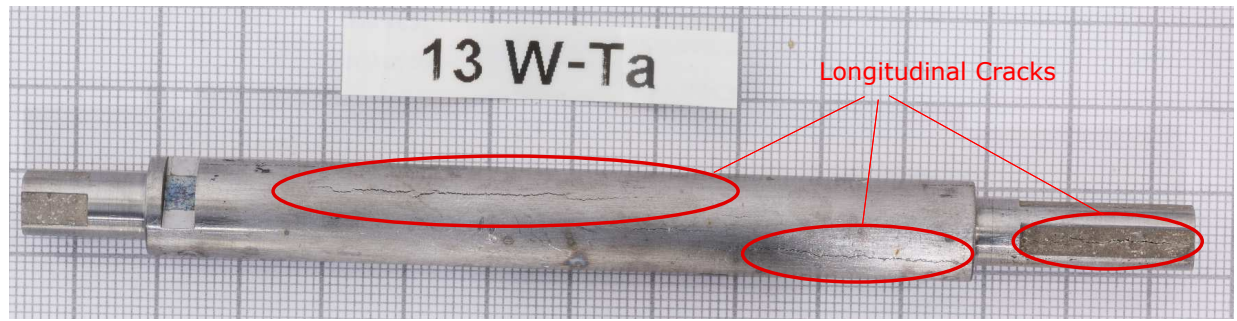

Figure 6.12: Picture of \#13WTa target after the experiment. This target was irradiated up to $1.62 \cdot 10^{12}$ ppp. (AD-Target conditions). Several longitudinal cracks, both in the tantalum cladding surfaces and in the un-cladded tungsten of the extremities can be observed.

It seems that the addition of the cladding did not improved the target response since several longitudinal cracks are present both in the tantalum cladding surfaces and in the un-cladded tungsten of the extremities. Ultrasound tests of the target also confirmed the presence of cracks in the bulk material. It is not fully explained why the tantalum material of the targets \#4Ta1 and \#5Ta2 did not crack, even when exposed to the direct proton beam impact, while tantalum of the cladding of this target did. A possible explanation is the large magnitude of hoop stresses reached in the Ta cladding due to the sudden expansion of the tungsten core. In 
addition, it must be mentioned that the properties of the tantalum used for the cladding can differ significantly in comparison to ones of the \#4Ta1 and \#5Ta2 targets due to differences in the manufacturing process and supplier.

\subsection{Graphite Clamps Damage}

As stated in the definition of objectives of the experiment, presented in section 4.2 of Chapter 4, it was not within its scope to assess the state of the graphite matrix surrounding the AD-Target core. However, it was shown by hydrocode simulations in section 3.8 of Chapter 3 that the stresses in this graphite matrix are also very high due to the propagation of the generated pressure wave in the high density core, threatening its structure.
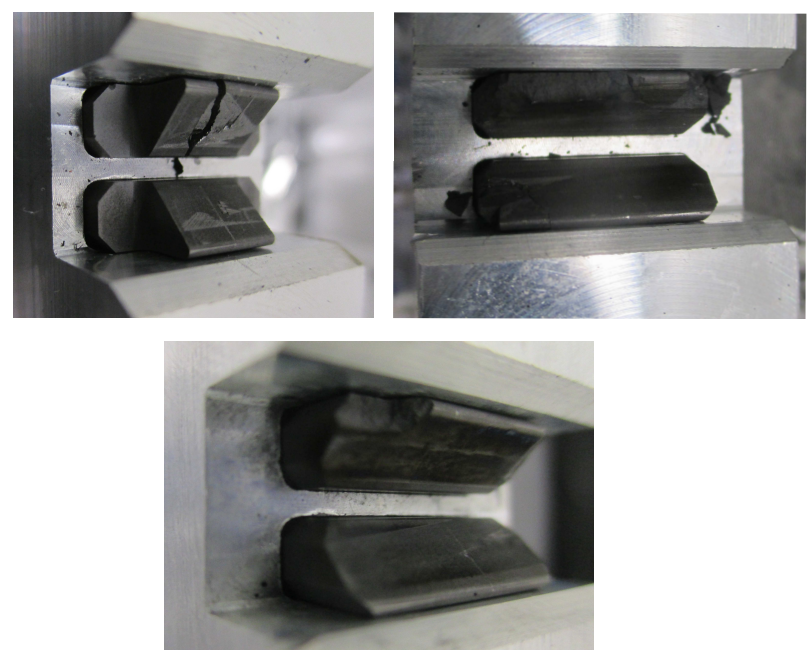

Figure 6.13: Fractured graphite clamps observed during the dismounting of the targets. This could give important hints of the state of the real graphite matrix in the AD-Target.

Nevertheless, even if the purpose of the experiment was not to check the graphite response, it was very interesting to observe during the dismounting of the targets after the experiment that several of the v-shaped graphite clamps were cracked, as shown in figure 6.13. This could give important hints of the state of real graphite matrix in the AD-Target core and its damage predicted by simulations and presented in figure 3.13. Also if one takes into account that the graphite in the clamps shown in the picture were supposed to have the ability to absorb part of the incoming wave thanks to the presence of the springs, while in the real $\mathrm{AD}$-Target the core is completely embedded and therefore is exposed to even more unfavorable conditions. 


\subsection{Measurements of Targets Deformation}

As observed in most of the pictures just presented, one of the most remarkable observations about the state of the targets at the end of the experiment is, apart from an extensive number of longitudinal cracks, the large level of bending deformation that they suffered. As described in section 5.2 of Chapter 5, this permanent deformation was probably caused by a bending mode excited due to minor off-axis in the impacting position which could have been up to $0.4 \mathrm{~mm}$. The excitation of these modes is simulated in the section 6.5 of the present chapter.

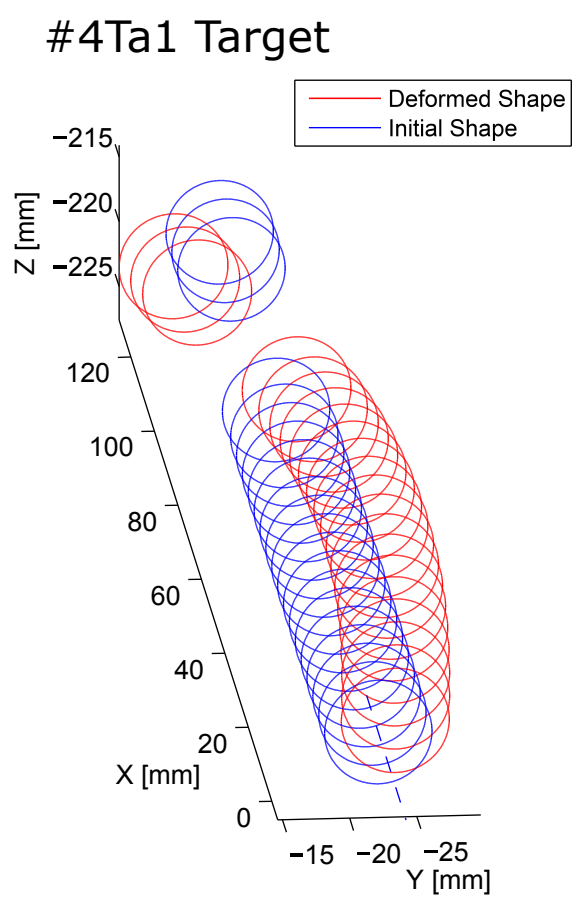

(a)

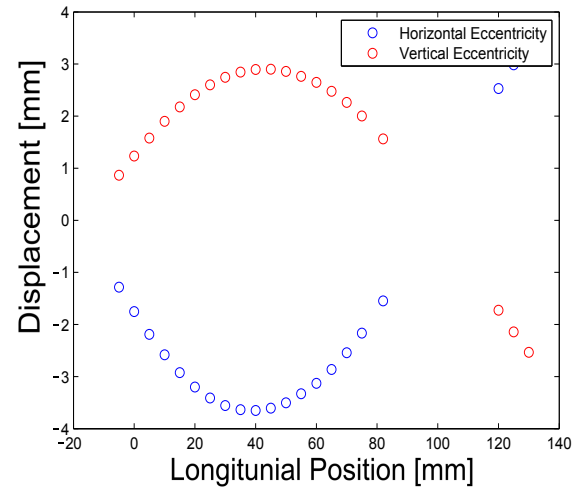

(b)

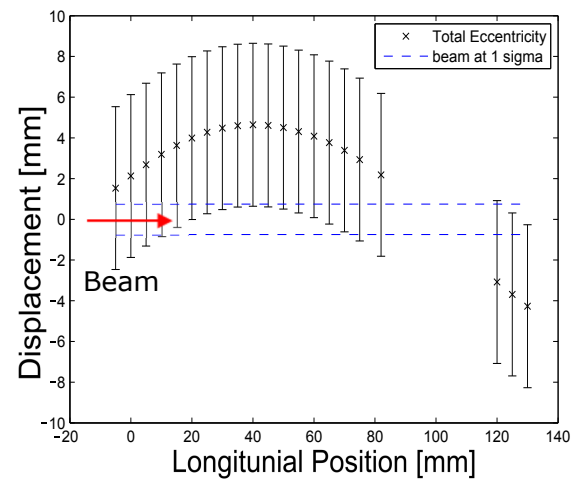

(c)

Figure 6.14: (a) Reconstruction of the deformed shape of the \#4Ta1 target after the experiment. (b) Horizontal and vertical eccentricity as a function of longitudinal position. (c) Plot of the total eccentricity of the tantalum target showing how the beam would barely interact with it.

In order to quantify the targets deformations, CMM measurements were done on the targets after the experiment before dismounting them from the sample holder. Hence, these measures could be compared with the ones which were taken before 
the experiment and used in the alignment procedure described in section 4.6.1 of Chapter 4. In this context, figures 6.14-6.16 show the reconstructed shapes of the targets and comparison with the original shapes of \#4Ta1, \#6W1 and \#10Ir respectively. In these figures it is plotted as well the external shape of the target together with a hypothetical incoming beam, which size at 1 sigma is indicated.

It is worth noting to mention that all the targets, no matter the material, deformed through the same direction (left and up with respect to the beam trajectory). This fact reinforces the hypothesis that this bending was provoked by a systematic minor error in the alignment which led to systematic off-axis impacts.

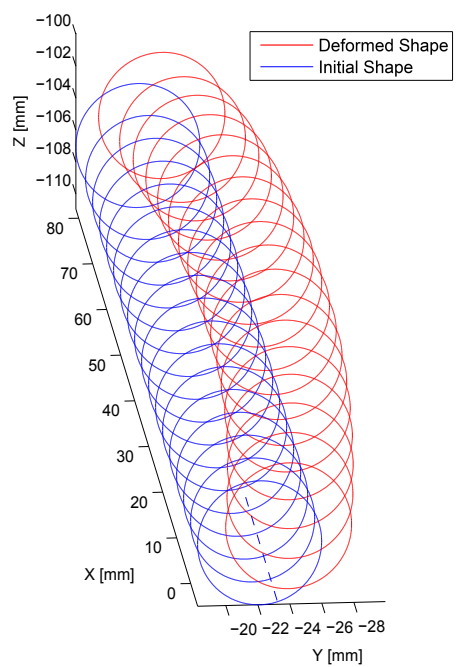

\section{\# 10Ir1 Target}

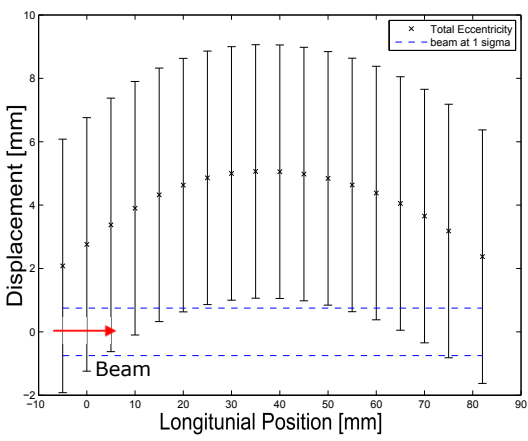

(b)

(a)

Figure 6.15: (a) Reconstruction of the deformed shape of the \#10Ir1 target after the experiment. (b) Plot of the total eccentricity of the iridium target showing how the beam would barely interact with it. This could explain why this target did not massively fragmented as \#12Ir3.

As can be seen in the plots of Figure 6.14-(c) and 6.15-(b), the large level of deformation of the tantalum and iridium targets at the end of the experiment (close to $5 \mathrm{~mm}$ ) caused that the beam halo was barely touching the targets periphery at their longitudinal center. These plots of course are showing a hypothetical situation since during the last impacted pulses at high intensities the target deformation was not yet reaching this level. But in any case, observing as well the eccentricities at intermediate states shown in Table 6.1, and from what it was observed online in the cameras during the experiment, it can be stated that before the last intensity pulses the deformation of these targets was already very advanced. This fact explains why the iridium target \#10Ir1 shown in figure 6.8 did 
not massively fractured as the \#12Ir3 of figure 6.8. The highest intensity beam was barely interacting with the target \#10Ir1 as shown by figure 6.15 .

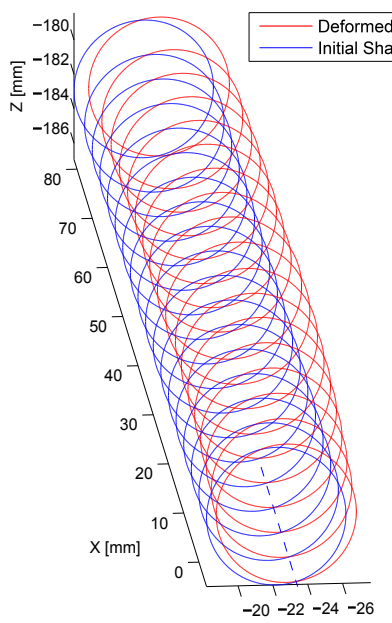

\#6W1 Target

(a)

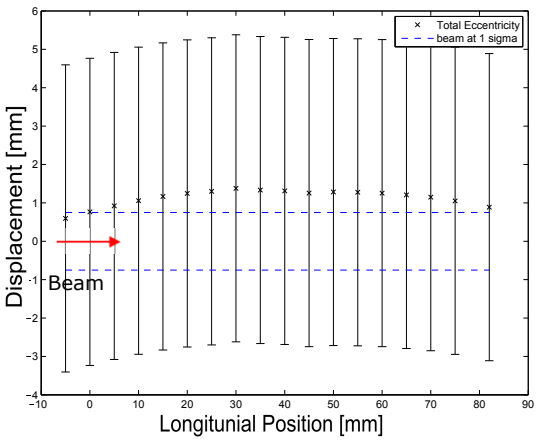

(b)

Figure 6.16: Reconstruction of the deformed shape of the \#6W1 target after the experiment. (b) Plot of the total eccentricity of the tungsten target. Tungsten targets presented much lower levels of bending deformation.

Finally, table 6.1 summarizes the maximum eccentricity reached at the end of the experiment in every single target. It can be observed that iridium and tantalum were the most sensitive materials to these bending deformations. Molybdenum and TZM were the least sensitive, probably due to lower and more homogenous longitudinal distribution of deposited energy because of their inferior density and maybe greater length. Tungsten seems to have a better response as well to this bending mode, especially taking into account its higher density and the high temperatures that were reached in its core. However, the tungsten target \#8W3 suffered from a very large deformation, probably caused by the first impacted beam at high intensity, as suggested by the progressive decrease of measured temperature shown by figure 5.19 of section 5.4.1. Finally, the tungsten target cladded in tantalum \#13WTa seems to have a better response as well, probably due to its increased robustness and moment of inertia provided by its $4 \mathrm{~mm}$ larger diameter in comparison with the rest of the targets. 
Table 6.1: Summary of maximum eccentricity of the irradiated targets measured after the experiment

\begin{tabular}{|c|c|c|}
\hline Target & $\begin{array}{l}\text { Maximum Intensity } \\
\text { Impacted [ppp] }\end{array}$ & $\begin{array}{l}\text { Maximum target eccentricity } \\
\text { at the end of the experiment } \\
{[\mathrm{mm}]}\end{array}$ \\
\hline$\# 1 \mathrm{Mo}$ & $\begin{array}{c}1.69 \cdot 10^{12} \\
\text { Full intensity }\end{array}$ & $x_{1}$ \\
\hline \#2TZM1 & $\begin{array}{l}1.67 \cdot 10^{11} \\
\text { Full intensity }\end{array}$ & 0.37 \\
\hline \#3TZM2 & $\begin{array}{l}9.22 \cdot 10^{11} \\
\text { Medium Intensity }\end{array}$ & 0.45 \\
\hline \#4Ta1 & $\begin{array}{l}1.67 \cdot 10^{12} \\
\text { Full intensity }\end{array}$ & 4.5 \\
\hline$\# 5 \mathrm{Ta} 2$ & $\begin{array}{c}9.22 \cdot 10^{11} \\
\text { Medium Intensity }\end{array}$ & 3.35 \\
\hline \#6W1 & $\begin{array}{l}1.65 \cdot 10^{12} \\
\text { Full intensity }\end{array}$ & 1.4 \\
\hline \#7W2 & $\begin{array}{c}4.84 \cdot 10^{11} \\
\text { Medium Intensity }\end{array}$ & 0.7 \\
\hline \#8W3 & $\begin{array}{c}4.84 \cdot 10^{12} \\
9 \text { pulses in a row }\end{array}$ & 4.8 \\
\hline \#9WLa & $\begin{array}{c}1.65 \cdot 10^{12} \\
\text { Full intensity }\end{array}$ & 1.9 \\
\hline \#10Ir1 & $\begin{array}{c}1.7 \cdot 10^{12} \\
\text { Full intensity }\end{array}$ & 5 \\
\hline$\# 11 \mathrm{Ir} 2$ & $\begin{array}{c}4.95 \cdot 10^{11} \\
\text { Single pulse }\end{array}$ & 0.9 \\
\hline$\# 12 \operatorname{Ir} 3$ & $\begin{array}{c}1.71 \cdot 10^{12} \\
\text { Full intensity }\end{array}$ & Fragmented \\
\hline \#13WTa & $\begin{array}{l}1.62 \cdot 10^{12} \\
\text { Full intensity }\end{array}$ & 0.56 \\
\hline
\end{tabular}




\subsection{Comparisons between Hydrocode Simulations and Experimental Results}

As mentioned in Chapter 4, one of the objectives of the thesis was to crosscheck the accuracy of the material models employed by the hydrodcodes, due to its inevitable extrapolation when applied to the AD-Target problem. In this section, comparisons between the simulated velocity at the targets' surface and experimental data is provided. This comparison is presented for three different regimes; (i) at the lowest intensity pulses, (ii) at middle intensity when internal cracking is already taking place, (iii) at the highest intensity pulses for the special cases of \#8W3 and \#12Ir3 targets.

These simulations received as input montecarlo FLUKA simulations which were taking into account the actual beam parameters impacted and recorded during the experiments, such as intensity, beam size (recorded by the BTV) and corresponding off-axis, which was also recorded by the BTV and presenting values from 0 to $0.4 \mathrm{~mm}$.

\subsubsection{Comparisons at low intensity pulses}

In this subsection numerical and experimental crosschecks are provided for $\mathrm{W}$, Ir and $\mathrm{Ta}$ in the lowest intensity pulses, at which the target materials did not experience yet a great amount of plastic deformation nor internal damage. For these simulations, no failure model is included in the simulations. However, the material response beyond plastic deformation is considered by the corresponding strength model.

\section{Tantalum}

Figure 6.17 shows the comparison between the simulated and recorded velocity for tantalum target \#5Ta2 when first impacted at low intensity $\left(1.1 \cdot 10^{11} \mathrm{ppp}\right)$. For this simulation, a J-C strength model extracted by Split Hopkinson bar tests up to $3800 \mathrm{~s}^{-1}$ and $1000{ }^{\circ} \mathrm{C}$ [78] was used. Mie-Grüneisen EOS is included. It is surprising the high level of a agreement between simulations and experimental velocity, in particular in terms of amplitude and frequencies during the first oscillations. This good agreement allows to rely on the stresses in the bulk material predicted by the simulations, at least at these conditions. The maximum adiabatic increase of temperature in this target at this impact is estimated to be in the order of $130{ }^{\circ} \mathrm{C}$. The oscillating strain rates reach values up to $6000 \mathrm{~s}^{-1}$.

The relative low increase of temperature (in comparison with further high intensity pulses) is already enough to induce plastic deformation in a wide region of the target, as shown in figure 6.18 , in which a plot indicating the areas where the 
reached von Mises stresses are above the yield strength $\sim 1 \mu$ s after the impact is presented. This plastic deformation apparently did not produce internal cracks in the material, as shown by the clear radial wave recorded and the preliminary PIEs.

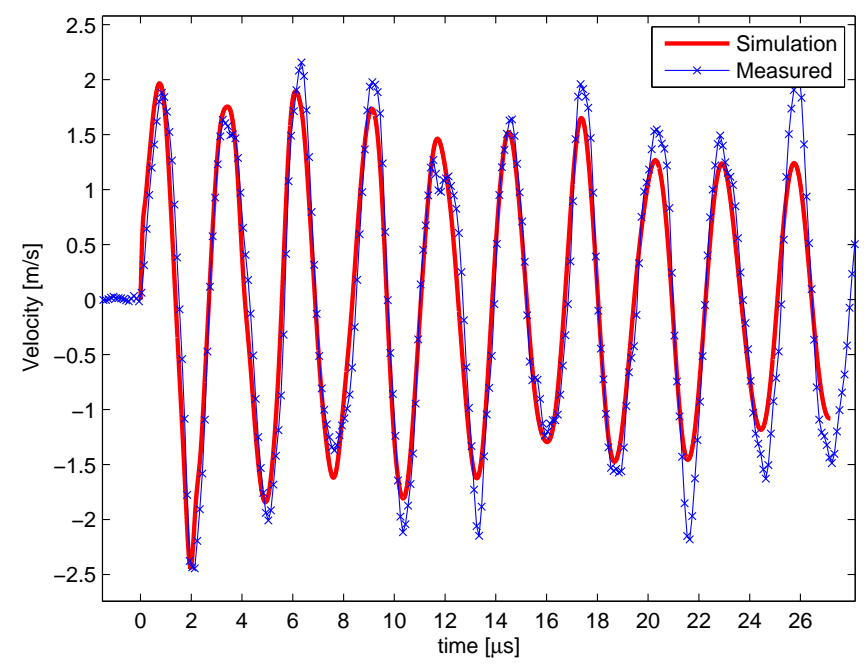

Figure 6.17: Recorded surface velocity vs hydrocode simulations of \#5Ta2 target impacted by $1.1 \cdot 10^{11} \mathrm{ppp}$. Tantalum response was simulated using a J-C strength model from ref. [78].

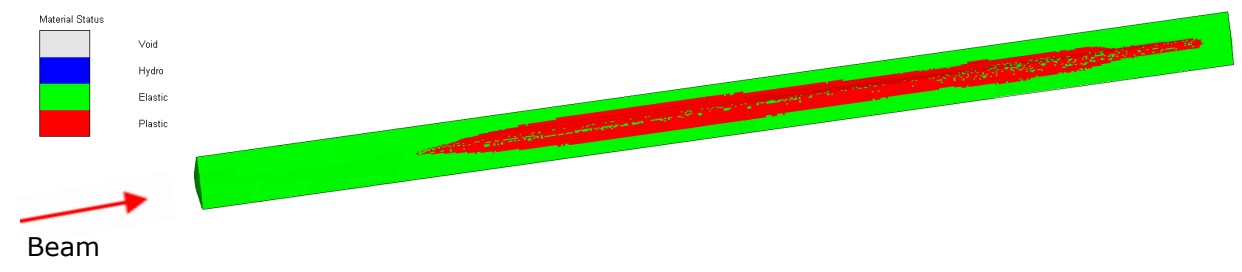

Figure 6.18: Contour plot showing the estimated region that suffered plastic deformation in the \#5Ta2 target at $\sim 1 \mu$ s after the impact of $1.1 \cdot 10^{11} \mathrm{ppp}$. 


\section{Tungsten}

In a similar way, figure 6.19 shows the comparison between the simulated and recorded velocity for \#6W1 target when first impacted at low intensity (1.09 . $\left.10^{11} \mathrm{ppp}\right)$. This simulation is considering a Z-A strength model recently extracted by dynamic testing in Politecnico di Torino up to $1200{ }^{\circ} \mathrm{C}$ and $10^{4} \mathrm{~s}^{-1}$. MieGrüneisen EOS is included. No failure model is included. Also in this case, the agreement between simulations and experimental velocity is very good, in particular in terms of amplitude and frequencies during the first oscillations. Accumulation of minor errors produces some frequency mismatches when the transient develops in time. Nevertheless, the high level of agreement in the radial wave velocity suggests the good aptitude of EOS and strength models employed by the simulations, allowing to be confident in the magnitude and nature of the predicted pressure wave inside the target material. The adiabatic increase of temperature in this target at these impact conditions is also estimated to be in the order of $140{ }^{\circ} \mathrm{C}$, producing a maximum tensile pressure of $380 \mathrm{MPa} \sim 0.9 \mu$ s after the impact. Figure 6.20 shows that plastic deformation is taking place already at these conditions, localized in the central longitudinal areas of the target where more energy is deposited. However, it must be mentioned that according the used Z-A fitted by dynamic testing, the predicted yield strength of tungsten is quite low, so the size of the region becoming plastic deformation may be over-predicted.

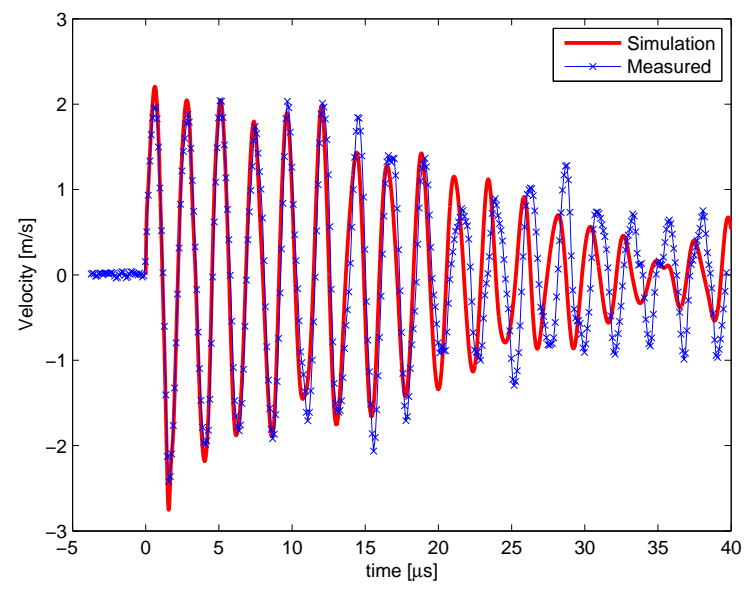

Figure 6.19: Recorded surface velocity vs hydrocode simulations of \#6W1 target impacted by $1.09 \cdot 10^{11} \mathrm{ppp}$. Tungsten response was simulated using a Z-A strength model recently extracted by dynamic testing in Politecnico di Torino. 


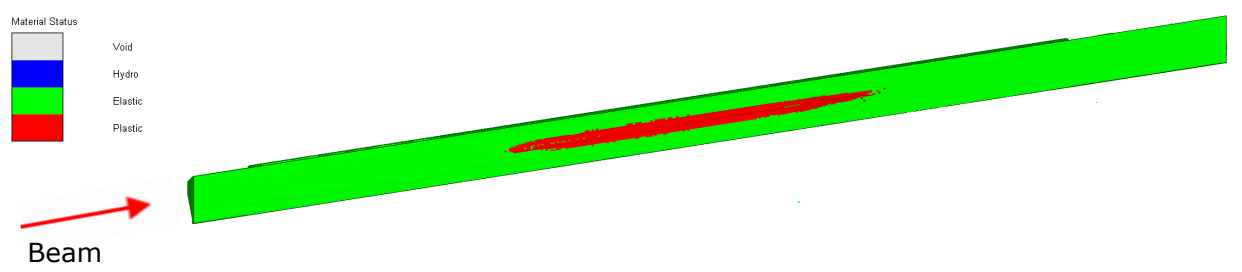

Figure 6.20: Contour plot showing the estimated region that suffered plastic deformation in the \#6W1 target at $\sim 1.2 \mu$ s after the impact of $1.09 \cdot 10^{11}$ ppp (first impacted pulse). This simulation used a Z-A strength model recently extracted. According to this model, the yield strength is very low, so the size of the region becoming plastic deformation may be over-predicted

\section{Iridium}

Finally, in figure 6.21 the comparison between the simulated and recorded velocity for \#11Ir2 when first impacted at low intensity $\left(1.15 \cdot 10^{11} \mathrm{ppp}\right)$ is shown. This simulation is considering a $\mathrm{J}-\mathrm{C}$ strength model recently extracted by dynamic testing in Politecnico di Torino up to $1200{ }^{\circ} \mathrm{C}$ and $10^{4} s^{-1}$. Mie-Grüneisen EOS is included. No failure model is included. The adiabatic increase of temperature in this target at these impact conditions is estimated in the order of $170{ }^{\circ} \mathrm{C}$, higher than previous cases due to iridium higher density.

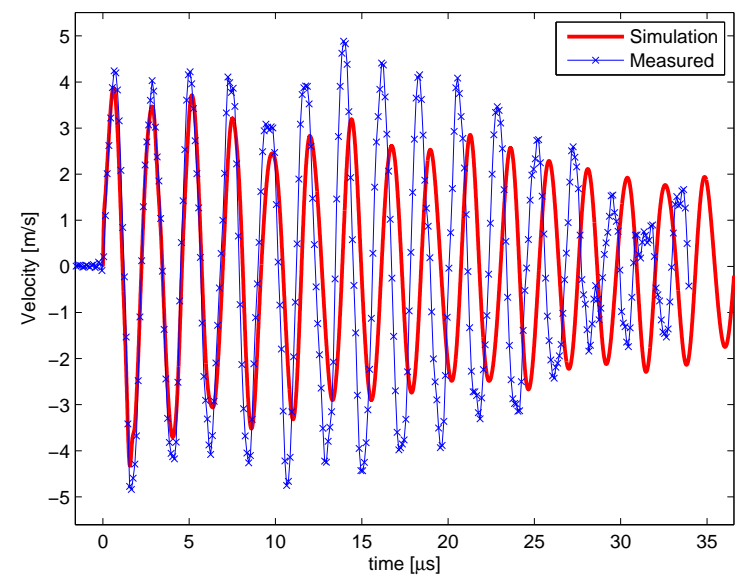

Figure 6.21: Recorded surface velocity vs hydrocode simulations of \#11Ir2 target impacted by $1.15 \cdot 10^{11} \mathrm{ppp}$. Tungsten response was simulated using a J-C strength model recently extracted by dynamic testing in Politecnico di Torino [67]. 
Again, very good agreement between simulations and experimental velocity is obtained, especially in amplitude and frequencies during the first oscillations. Accumulation of minor errors produces frequency mismatches when the transient develops in time above $12 \mu \mathrm{s}$. A possible reason of this is that, according to the strength model used in the simulations, no plastic deformation is taking place yet, while it could be the case in reality, in particular when comparing what predicted for tungsten. It is very interesting as well to observe the higher velocities recorded and predicted for iridium in comparison to tungsten (almost the double during first oscillation). This is due, not only to the higher deposition of energy in iridium, but also for its significant higher thermal expansion coefficient. The prediction of these expansions and velocities reinforced the good performance of EOS employed, in which the thermal expansion is taking into account through the Grüneisen parameter.

\subsubsection{Medium Intensities and Failure Model Benchmarking}

Comparisons between experimental data vs simulations at more demanding conditions (higher intensity pulses) is presented in this subsection for tantalum, tungsten and iridium. As one can imagine, the numerical prediction in such scenarios is more challenging, since cracking and permanent changes in the targets material was already taking place. Nevertheless, this effect was taken as a chance for bench-marking failure models. This was done by performing several simulations per scenario assuming different values of $\mathrm{P}_{\text {min }}$ at which the material would crack and therefore do not withstand further tensile or shear stresses.

\section{Tantalum}

Figure 6.22 shows the experimental velocity vs numerical comparison for \#4Ta1 target at the $2^{\text {nd }}$ irradiation intensity $\left(2.15 \cdot 10^{11} \mathrm{ppp}\right)$. As can be observed in the figure, very good agreement between simulations and experiment is still achieved, indicating the modeled conditions are representative. Maximum adiabatic rise of temperature associated to this impact is estimated to be $415^{\circ} \mathrm{C}$. No failure model is included in the simulations. Maximum strain rate reached is $6 \cdot 10^{3} \mathrm{~s}^{-1}$ and maximum tensile pressure is in $-820 \mathrm{MPa}$. It is very interesting to see that at these conditions, a great amount of the target has suffered plastic deformation, as it is shown by figure 6.23. As anticipated in the case at the lowest intensity, this plastic deformation does not seem to induce cracks in tantalum. 


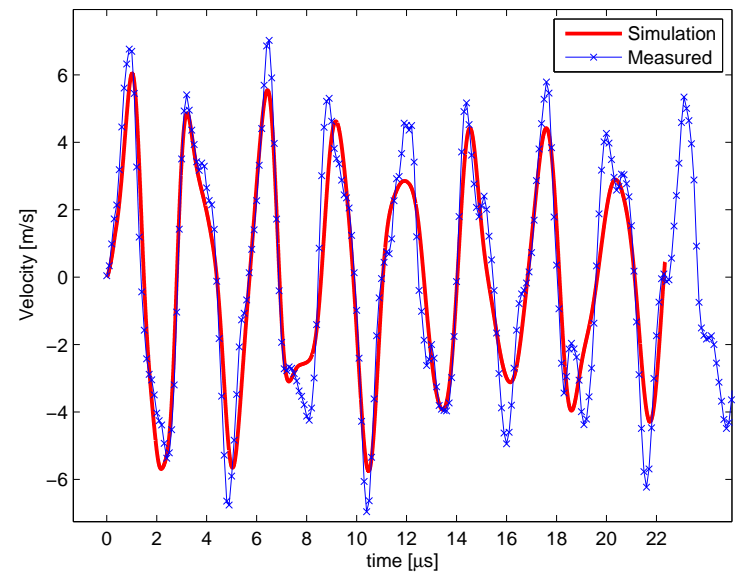

Figure 6.22: Recorded surface velocity vs hydrocode simulations of \#4Ta1 target impacted by $2.15 \cdot 10^{11}$ ppp. Tantalum response was simulated using a J-C strength model from ref. [78].

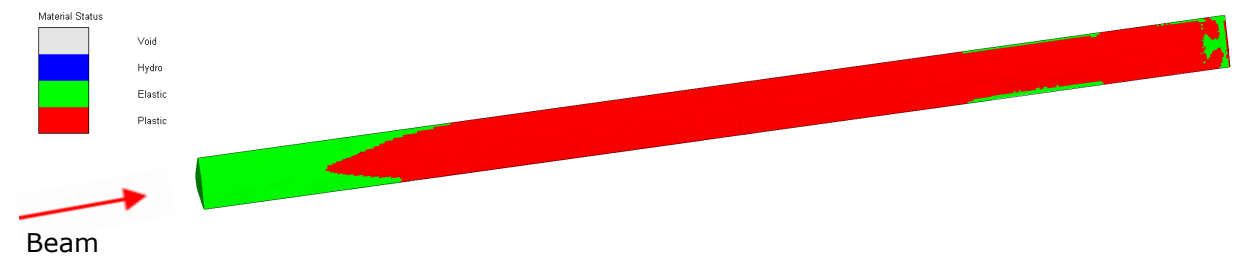

Figure 6.23: Contour plot showing that a great amount of the \#4Ta1 material suffered plastic deformation from the $2^{\text {nd }}$ irradiation intensity at $2.15 \cdot 10^{11} \mathrm{ppp}$.

\section{Tungsten}

Figures 6.24(a)-(b) show experimental data vs simulations comparisons for the first pulse at $2.17 \cdot 10^{11} \mathrm{ppp}\left(2^{\text {nd }}\right.$ irradiation intensity) impacted onto the \#6W1 target. In figure (a) the numerical simulations is not considering any failure model while in (b) a minimum hydrostatic pressure failure model has been bench-marked, finding a value $\mathrm{P}_{\min }=-850 \mathrm{MPa}$ as the one that better fits the experimental data. As can be seen in the plot, the addition of the failure model in the simulation provides a great improvement, in particular for the prediction of the recorded fast damp a distortion of the radial wave. This accurate prediction when including internal damage in the simulations reinforces the hypothesis drawn in Chapter 5 , suggesting that the distortion of the radial wave recorded was symptom of internal cracking, rather than just hardening due to plastic deformation. It is important 
to mention that this distortion in tungsten was observed from the $2^{\text {nd }}$ irradiation intensity (conditions 7 times lower as the ones reached in the AD-Target).

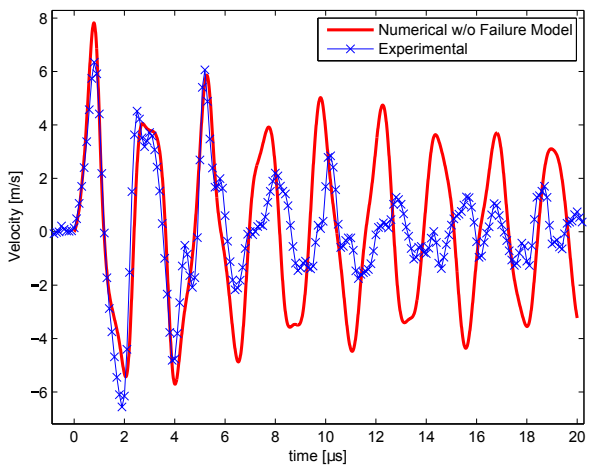

(a)

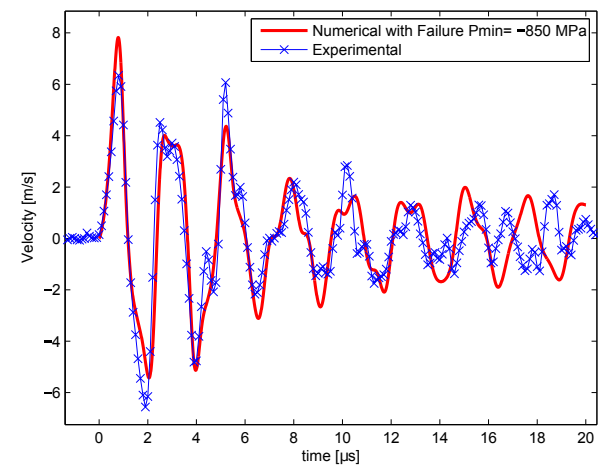

(b)

Figure 6.24: Recorded surface velocity vs hydrocode simulations of the \#6W1 target impacted by $2.17 \cdot 10^{11} \mathrm{ppp}$ (a) Without considering a failure model in the simulations. (b) Considering a failure model with $\mathrm{P}_{\min }=-850 \mathrm{MPa}$

Figure 6.25 shows a snapshot of the internal damage predicted at the target \#6W1 after impacted by $2.17 \cdot 10^{11} \mathrm{ppp}$. The adiabatic increase of temperature associated to this failure is in the order of $530{ }^{\circ} \mathrm{C}^{2}$. Strain rates in the order of $6 \cdot 10^{3} \mathrm{~s}^{-1}$ were reached in the central failed areas while up to $3 \cdot 10^{3} \mathrm{~s}^{-1}$ were reached in the surrounding, not failed, areas. Extensive plastic deformation is predicted in the areas surrounding the failure.

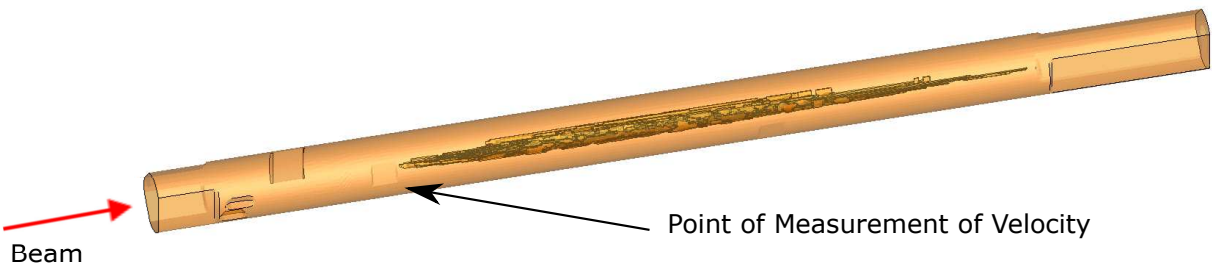

Figure 6.25: Contour plot of the predicted state of the \#6W1 target after impacted by $2.17 \cdot 10^{11} \mathrm{ppp}$, showing the failed region. The threshold for failure was set in $\mathrm{P}_{\min }=-850 \mathrm{MPa}$ consistently with the bench-marked response shown in figure 6.24-(b).

\footnotetext{
${ }^{2}$ This is considerably higher than the double of the $130{ }^{\circ} \mathrm{C}$ of $\Delta T$ previously presented in section 6.4 .1 (associated to $1.17 \cdot 10^{11} \mathrm{ppp}$ ) since in these first pulses at low intensity the beam sizes were considerably higher than the ones requested (around $1.8-2 \mathrm{~mm}$ at $1 \sigma$ ) while in the $2^{\text {nd }}$ irradiation intensity they were more focused (around $1.1 \mathrm{~mm}$ at $1 \sigma$ ).
} 


\section{Iridium}

Similarly as with tungsten, figure (a)-(b) show the experimental data vs simulations comparisons for the first pulse by $2.22 \cdot 10^{11} \mathrm{ppp}\left(2^{\text {nd }}\right.$ irradiation intensity) impacted onto the \#10Ir1 target. In figure (b) the failure model has been benchmarked to a value of $-900 \mathrm{MPa}$, achieving with this value the best fitting. It is remarkable the prediction of magnitude of damping and distortion of the radial wave when adding the failure model, in particular after the $3^{\text {rd }}$ oscillation.

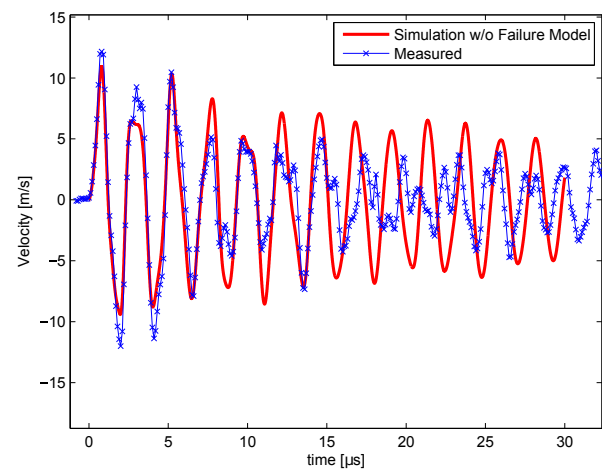

(a)

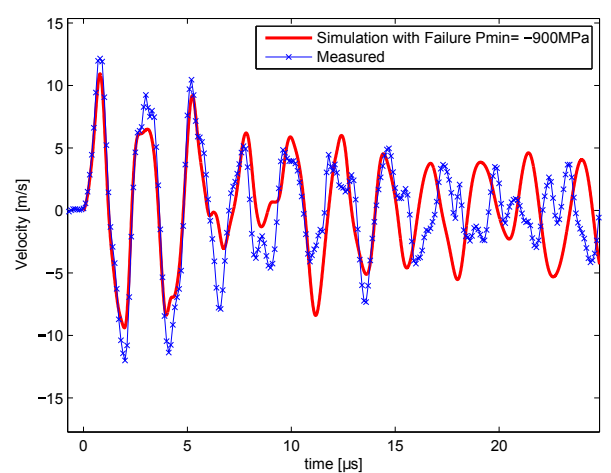

(b)

Figure 6.26: Recorded surface velocity vs hydrocode simulations of target \#10Ir1 impacted by $2.22 \cdot 10^{11} \mathrm{ppp}$ (a) Without considering a failure model in the simulations.(b) Considering a failure model with $P_{\min }=-900 \mathrm{MPa}$

The simulation with the bench-marked model predicts an adiabatic rise of temperature of $530{ }^{\circ} \mathrm{C}$, similar as in tungsten. The strain rate oscillates, reaching a maximum in the order of $8 \cdot 10^{3} s^{-1}$ in the center and $4 \cdot 10^{3} s^{-1}$ in the periphery. Figure 6.27 shows a snapshot of the internal damage predicted in the iridium target at these conditions, which are still several times lower as the ones reached in the AD-Target core.

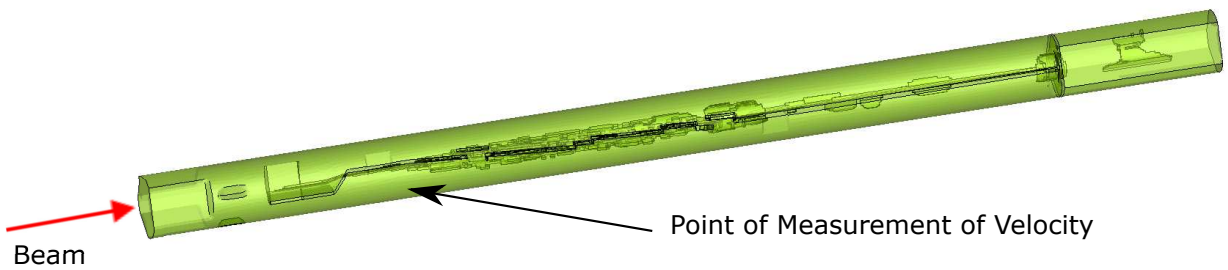

Figure 6.27: Contour plot of the predicted state of the \#10Ir1 target after impacted by $2.22 \cdot 10^{11} \mathrm{ppp}$, showing the failed region. The threshold for failure was set in $P_{\min }=$ $-900 \mathrm{MPa}$. 


\subsubsection{Comparisons at High Intensity Pulses for \#8W3 and \#12Ir3 targets}

Finally, comparisons between experimental and simulated target velocity is presented for two additional pulses, corresponding to the special cases in which a high intensity pulse (recreating AD-Target conditions) was impacted in the targets \#8W3 and \#12Ir3, which were not previously irradiated during the experiment. A benchmarking of the failure model has been done in the same way as in the cases just presented in the previous sections.

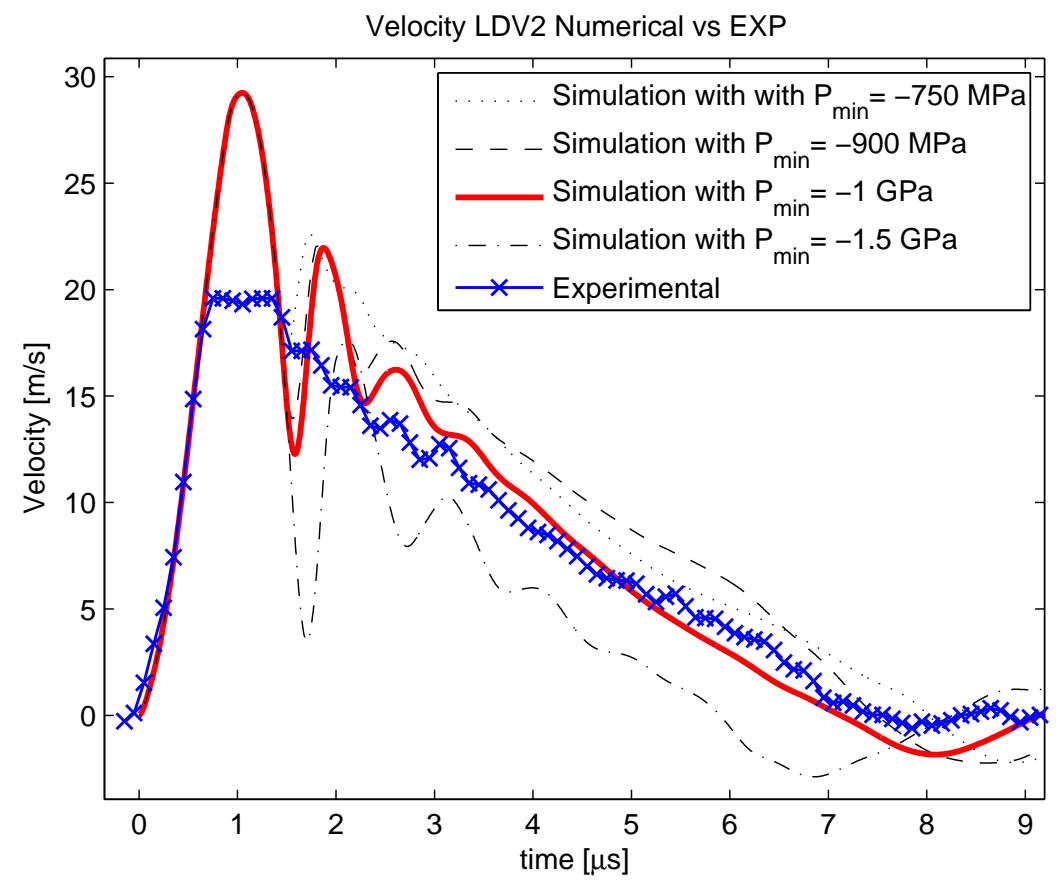

Figure 6.28: Recorded surface velocity vs hydrocode simulations of target \#8W3 impacted by $1.67 \cdot 10^{12} \mathrm{ppp}$ (similar AD-Target conditions). Several simulated response considering different threshold for failure are presented, being $P_{\min }=-1 \mathrm{GPa}$ the one that achieves the best prediction.

Figure 6.28 shows the failure model fitting performed to the velocity recorded during $9 \mu$ s when impacting a pulse of $1.67 \cdot 10^{12}$ ppp for the first time. This pulse case was already introduced in figure 5.18 of section 5.4.1 in Chapter 5. It can be see that the LDV saturated above $20 \mathrm{~m} / \mathrm{s}$ (its velocity limitation), not being able to record the peak of velocity. 
In the plot, several simulations with different values of $P_{\min }$ for failure threshold from $-750 \mathrm{MPa}$ to $1.5 \mathrm{GPa}$ are shown. All of them predict the same first rise of velocity (since material is not yet failing), reaching up to $30 \mathrm{~m} / \mathrm{s}$. The tendency of the simulated velocity fits very well the experimental data recorded before saturation of the LDV. After the peak, it can be seen that the simulation considering $\mathrm{P}_{\text {min }}=-1 \mathrm{GPa}$ achieves the best prediction. In this simulation a second peak of velocity is predicted, caused by the reflection of the radial wave in the interface with the central area already fragmented. However, this second peak was not recorded.

Figure 6.29 shows the snapshot of the internal damage predicted in the target at these conditions of impact, showing that the majority of internal volume fails. The maximum adiabatic increase of temperature associated to this pulse is in the order of $1680{ }^{\circ} \mathrm{C}$. This temperature is lower than the initially foreseen of $2000{ }^{\circ} \mathrm{C}$ since the real beam size obtained was $1.8 \mathrm{~mm}$ at $1 \sigma$ instead of $1.5 \mathrm{~mm}$. Maximum strain rate reached in the first oscillation before fracture is estimated in $2.5 \cdot 10^{4} \mathrm{~s}^{-1}$.

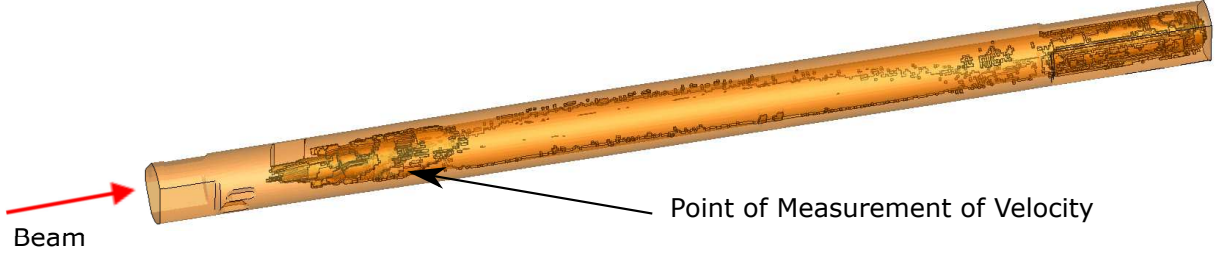

Figure 6.29: Contour plot of the predicted state of the \#8W3 target after impacted by $1.67 \cdot 10^{12} \mathrm{ppp}$, showing the failed region, which covers almost all the internal volume. The threshold for failure was set in $\mathrm{P}_{\min }=-1 \mathrm{GPa}$, consistent with the best data fitting obtained in figure 6.28 .

In a similar way, figure 6.30 shows the failure model benchmarking for the velocity recorded in the pulse at the highest intensity impacted onto the \#12Ir3 target, which provoked the massive fragmentation presented in the picture of figure 6.10. To fit this response via hydrocodes simulations was more difficult than for the case of $\# 8 \mathrm{~W} 3$, probably due to the peculiarities of this massive fragmentation involving large deformation and effects not taken into account by the code, which could have played an important role in the fracture, such as the anisotropy of the material caused by the manufacturing process. The minimum hydrostatic pressure failure model used may be too simple to predict all these phenomena. Saturation of the LDV recordings above $20 \mathrm{~m} / \mathrm{s}$ makes also impossible to know which was the maximum surface velocity reached. Nevertheless, different simulations with tensile pressure thresholds between -1.2 and -1.7 GPa have been carried out and are presented in figure 6.30. The best data prediction was achieved by assuming a failure pressure of $-1.4 \mathrm{GPa}$. Sudden rise of velocity and its drop after 5-10 $\mu \mathrm{s}$ is predicted, as well as intermediate oscillations after the first velocity peak, which 
would reach up to $50 \mathrm{~m} / \mathrm{s}$ according to simulations. However, in the experimental data recorded, these oscillations went down to velocities of a $5 \mathrm{~m} / \mathrm{s}$, while in the simulated response they are staying above $30 \mathrm{~m} / \mathrm{s}$. The experimental data also showed that after the drop of velocity, a stabilization took place around $10 \mathrm{~m} / \mathrm{s}$ for at least $50 \mu \mathrm{s}$ (meaning that the external face of the target was progressively detaching), while in the simulations velocity drops faster well below $5 \mathrm{~m} / \mathrm{s}$ in only $20 \mu s$.

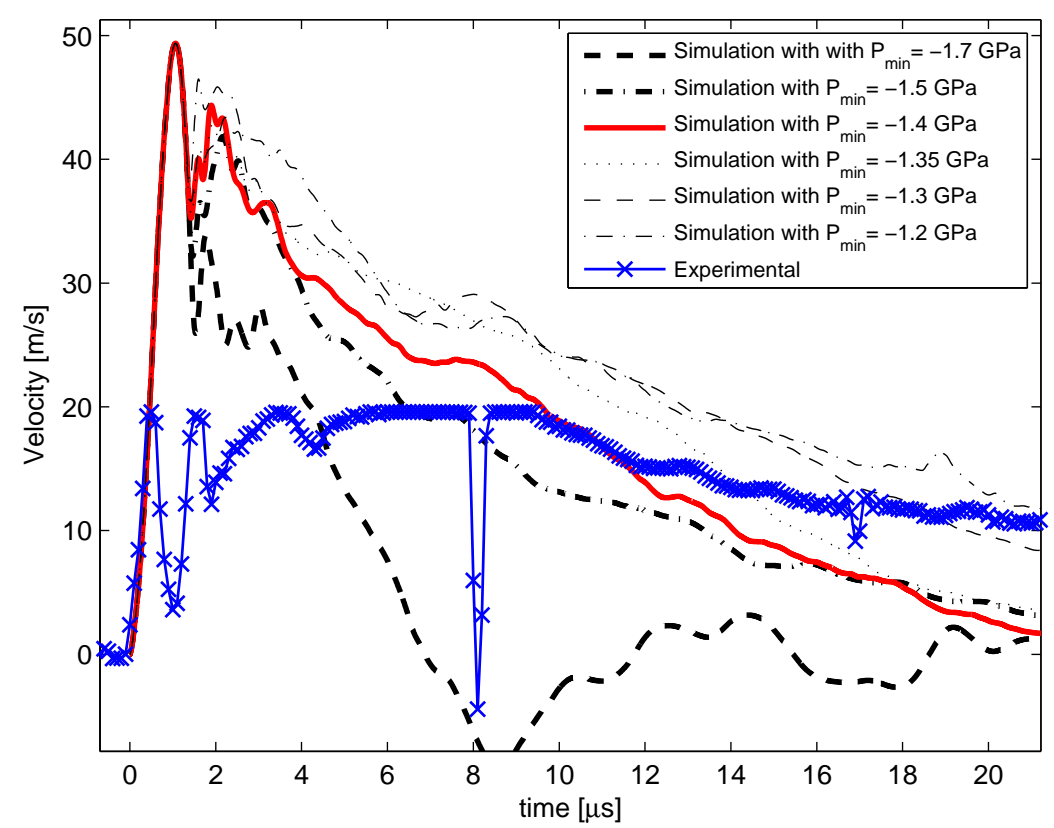

Figure 6.30: Recorded surface velocity vs hydrocode simulations of target \#12Ir3 impacted by $1.71 \cdot 10^{12} \mathrm{ppp}$ (similar to AD-Target conditions). Several simulated responses considering different thresholds for failure are presented, being $\mathrm{P}_{\min }=-1.4 \mathrm{GPa}$ the one that achieves the best prediction.

Finally, figure 6.31 shows the snapshot of the predicted state of the \#12Ir3 target $100 \mu s$ after the impact, assuming a failure threshold of $-1.4 \mathrm{GPa}$. The maximum adiabatic increase of temperature associated to this pulse is in the order of $2000{ }^{\circ} \mathrm{C}$ as is shown already in figure 6.11. Maximum reached strain rates are in the order of $4 \cdot 10^{4} \mathrm{~s}^{-1}$. It is very interesting to observe in the snapshot how the mode of failure shown in figure 6.10 is at least quantitatively recreated, presenting the partial detachment of the external regions following this sort of filament shape. It is important to note that this mode of failure is recreated without including any 
material anisotropy in the model, suggesting that it could be more governed by the type of load rather than the manufacturing process of the rod.

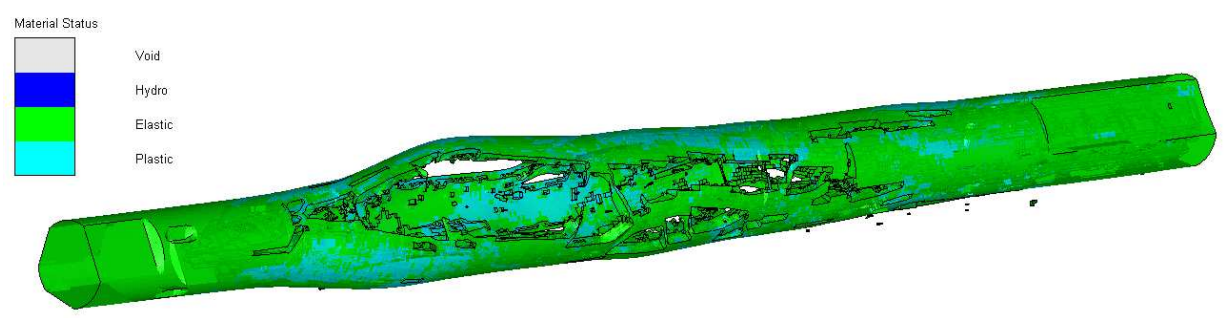

Figure 6.31: Contour plot of the predicted state of the \#12Ir3 target $100 \mu$ s after impacted by $1.71 \cdot 10^{12} \mathrm{ppp}$, showing detachment of external surfaces as observed in reality in figure 6.10 . The threshold for failure was set in $P_{\min }=-1.4 \mathrm{GPa}$, consistent with the best data fitting obtained in figure 6.30.

\subsection{Simulations of Targets Bending and Experimental Crosscheck}

The targets bending taking place during the experiment was a no foreseen phenomenon during the experiment design. As it was remarked in section 6.3 , this permanent bending was observed for all the irradiated targets. In addition, it interesting to note that this bending followed a similar direction for all the targets. The low frequency and high amplitude wave recorded by the LDV and interferometers and presented in section 5.2 of Chapter 5 represented an important hint regarding the origin of this bending deformation. It was already discussed that a bending mode could have been excited due to offsets in the beam impact position with respect to the target or asymmetry of the clamping system.

It was considered interesting to delve into the origin of this phenomenon and validation of the aforementioned hypotheses in order find possible improvements in future experiments or to apply the acquired knowledge when using targets in form of rods. The method for validating the hypothesis was to try to simulate the recorded displacement and, once this is done, to learn about the physics behind it and find strategies to mitigate this effect. For this reason, it was necessary to build a model of the target and the clamping system as much realistic as possible. In that sense, hydrocode simulations using Autodyn $\AA$ were not the most suitable tool since the clamping system (including contact algorithms and springs) cannot be recreated. In addition, the small time steps (below nanoseconds) required by the CFL (Courant-Friedrichs-Lewy) condition and numerical stability of the explicit solver of Autodyn (B) would have led to very long simulations to reach the milliseconds period of the recorded wave. On the other hand, ANYSY® Mechan- 
ical was found to be suitable for this purpose, since its implicit solver can deal with larger time steps and there is a wide number of types of boundary conditions that can be implemented, as well as spring elements.

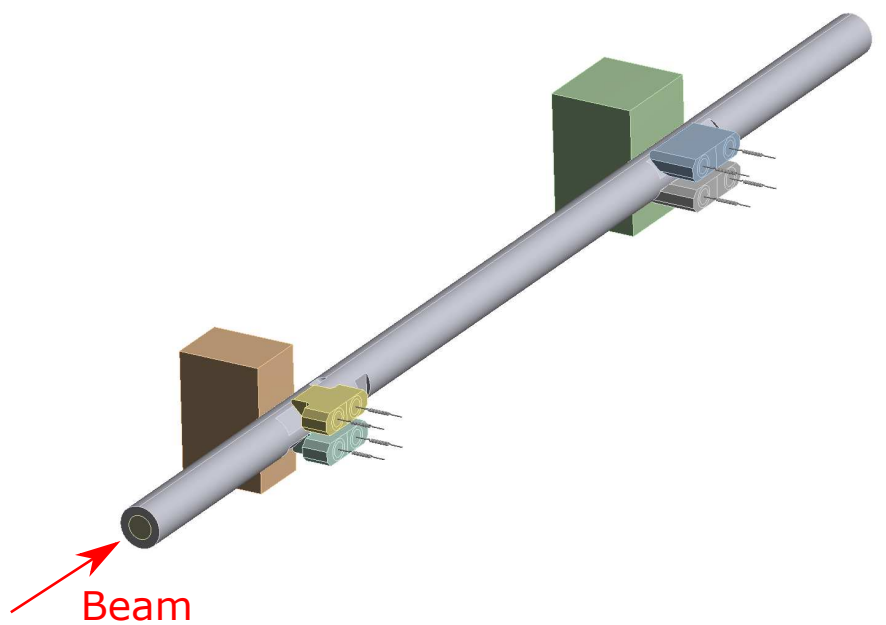

Figure 6.32: Geometry of the ANYSY® model of the TZM target and graphite clamping system (including springs) used for recreating of the observed bending mode.

Figure 6.32 shows the geometry employed for the simulations. The geometry selected corresponds to one of the TZM targets, since it was in these targets where the lower frequency wave was more clearly recorded. In addition, the lower density of this material with respect the other targets produced a response within the elastic regime at low intensity pulses, which was considered the most appropriate for being recreated since it avoids further uncertainties on the material response beyond plastic deformation. Four spring elements with the realistic elastic modulus and compressions ratio as the ones present in the experimental setup were included in the model. Boundary conditions were added on the horizontal planes of the graphite clamps in order to constrain their displacement in the vertical direction in the same way as the aluminum frame of the sample holder was doing. Frictionless contacts between the graphite clamps and the rod surfaces were included so there is not physical bonding between the clamps and the target.

Simulations were carried out in two steps. First, a thermal transient simulation was performed by importing the deposited energy in the target, which was estimated by FLUKA simulations. The beam parameters in terms of beam size and offsets of this FLUKA simulation were consistent with the ones recorded by the BTV during the experiment, in particular with the first beam impacted onto the \#3TZM2 target, which had an intensity of $1.2 \cdot 10^{11} \mathrm{ppp}$ a beam size of $1.8 \mathrm{x} 2 \mathrm{~mm}$ at $1 \sigma$ 
and an offset of $\sim 0.4 \mathrm{~mm}$ in the vertical plane. The experimental data used for the simulations crosschecks also correspond with this pulse. The second part of the simulation consisted in a transient structural up to $300 \mathrm{~ms}$ duration where the temperature field of the thermal simulation (adiabatic rise of temperature due to the beam impact) was applied. In order to have a reasonable simulation time, the time step selected for the transient simulation was progressively increasing up to $10 \mu \mathrm{s}$ after the first $15 \mu \mathrm{s}$ during which the waves started developing. This means that there is a loss of information of the faster phenomena such as the radial waves which are not taken into account in the simulation. Nevertheless, as it will be seen, the results still had physical meaning and slower phenomena such as the longitudinal waves and bending modes could be observed.

The computational model also included the mirror at which the LDV was pointing, so the simulated displacement at this point could be directly compared with the data recorded. First simulations carried out using the beam offsets recorded by the BTV did not show the observed bending wave with a clear and sufficiently identifiable amplitude. At this point, it is important to remark that the center of the BTV was not necessarily the center of the target since, even though they were aligned by means of meteorology measurements and laser trackers as described in section 4.6.1, this alignment involved several movable parts and components and it is not inconceivable that at the time of the experiment, the BTV-Target alignment was not as accurate as initially foreseen. This lead to the hypothesis that an unmeasured systematic offset on the impact position was taken place. For this reason, several parametric simulations were carried out considering small offsets in the horizontal plane with respect to the impact position shown by the BTV recordings.

Figure 6.33 shows the simulated displacement for several cases of the introduced parametric analysis, together with the experimental data recorded. As can be observed, the bending mode was not excited when the beam offset recorded by the BTV was simulated (which corresponds to $\sim 0$ offset in the horizontal plane). However, a wave with a similar period of the one recorded appeared in the simulations that were considering horizontal offsets from $0.35 \mathrm{~mm}$. Moreover, a similar amplitude of $14 \mu \mathrm{m}$ as the one recorded was achieved with a simulation considering an offset of $0.575 \mathrm{~mm}$. The wave period and amplitude match strongly confirm that, indeed, the origin of the lower frequency recorded wave was the excitation of a bending mode due to beam impact offset. This offset, found in the order of only $0.5 \mathrm{~mm}$, is compatible with a possible BTV-Target misalignment if one takes into account that they were separated by more than $1 \mathrm{~m}$. Another important remark that can be observed in the parametric analysis is the great sensitivity in the displacement response to small variations in the impact offset. This could explain the limited repeatability observed in the experimental data recorded in terms of amplitude of this lower frequency bending mode for different pulses and targets. 


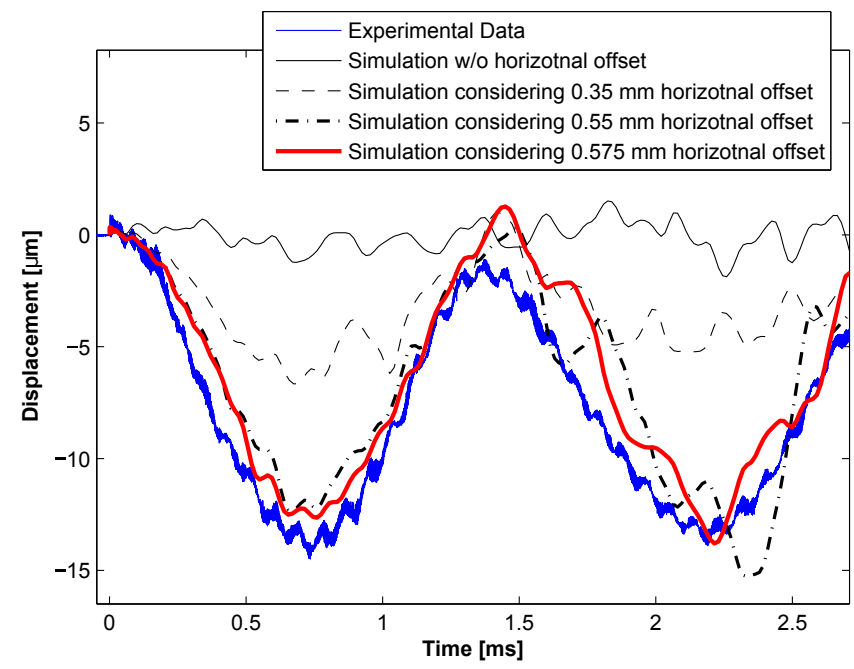

Figure 6.33: Comparison of the displacement measured experimentally by the LDV and the simulated displacement considering different beam impact offsets in the horizontal plane. Courtesy of N. Solieri.

To conclude, a very important lesson taught by the measurement of this bending mode and the numerical analysis performed to find its origin, is its appearance from a very small misalignment in the impact position. For the particular case of the TZM target studied, it was found that only a $0.5 \mathrm{~mm}$ offset (which corresponds to $6 \%$ of the rod radius) was sufficient to excite it. This was not expected during the design phase and must be taken into account for future experiments as well as general beam intercepting devices when using thin rods as targets.

\subsection{Main Outcomes of the Experiment and Proposed Improvements}

Chapters 4, 5 and 6 of this thesis present a detailed description of the HRMT-27 -Rodtarg experiment, including its motivation, goals, design, online results, first PIEs and simulation vs experimental comparisons.

As stated in Chapter 4, the motivation of the experiment was to solve the uncertainties existing in the response of the AD-Target core, to understand the level of the destructive effect of the generated pressure wave and its potential influence in the antiproton production. Based on that, the goals of the experiment were to recreate the $\mathrm{AD}$-Target conditions in different high density targets, measuring online data that could be contrasted with the predictions of simulations and try to 
identify the limits of the materials of interest at these conditions. In addition, another goal was to search for a high density material that could behave significantly better than the current core material.

Even if the goals of the experiment were very ambitious, it can be stated that the experiment has been a success and most of these goals have been fulfilled. The conditions reached in the $\mathrm{AD}$-Target core and the generated radial waves by the beam impact were recreated while being able to record extensive online data. This data was extremely useful, not only for comparisons with simulations, but because it was already telling online when the internal damage in the materials was taking place by the observed distortion of the radial wave. The particular geometry of the experimental targets and used beam parameters, based on thin rods and short pulses which could greatly excite these radial waves or modes, was demonstrated very useful for this purpose. The change and distortion of the radial wave is so clear that this experimental methodology could be also useful for other general experiments, even non-related to accelerator technology, to learn about fracture limits of materials at very high temperatures and strain rates.

The change and distortion of the measured radial wave indicated that all the materials except tantalum suffered internal cracking from low irradiation intensities, associated to rises of adiabatic temperatures from $200{ }^{\circ} \mathrm{C}$ for Mo and TZM $\left(3^{\text {rd }}\right.$ irradiation intensity) up to $530{ }^{\circ} \mathrm{C}$ for $\mathrm{W}$ and $\mathrm{Ir}\left(2^{\text {nd }}\right.$ irradiation intensity). This is well confirmed already by the preliminary PIEs which show the extended damage of the targets after the experiment as well as by the great agreement between experimental and numerical simulations, in particular when adding failure models which predict this radial wave distortion. The consequence of this early failure in the irradiated materials (in particular of Ir and W) is that even the simulations presented in Chapter 3, which were predicting a significant internal damage in the AD-Target core, were considerably underestimating this damage since in these simulations it was assumed a $P_{\min }=-2.3 \mathrm{GPa}$ for $\mathrm{W}$ (taken from spall strength experiments), while the experiment demonstrated that this internal fracture may be taking place from -850 MPa and -900 GPa for W and Ir respectively. By looking at the state of the \#12Ir3 target, massively fragmented just after the pulse impact at AD-Target conditions, one can imagine the extend of internal fracture of the real AD-Target iridium core. This fracture will definitely affect its effective density and antiproton production. This drop in production may not be even noticed by operation as is taking place from the very beginning. Drops of antiproton production of $20 \%$ have been reported after a few days of operation, which could be just consequence of the extension of this internal damage and dispersion of the cracked material towards the periphery of the core (taking also into account that the graphite matrix may be also cracked). This phenomenon, if confirmed, could leave room for a great improvement in antiproton production if the target material withstands the proton beam impacts without fragmenting. Foreseen opening of a spent AD-Target could confirm this hypothesis. 
It has been found very good agreement between hydrocode simulations and experimental data recorded, even at high intensity pulses. It is interesting to see that this agreement takes place even if the strength models are partially extrapolated as stated in Chapter 3. For example, in figure 6.28 of section 6.4.3 very good agreement was achieved in $\mathrm{W}$ impacted at the highest intensity even if the reached strain rates at these conditions were in the order of $2.5 \cdot 10^{4} \mathrm{~s}^{-1}$, well above the ones at which the used strength model was extracted. Two possible explanations for this have been found; one is that the effect of thermal softening in the strength of the material due to the high temperatures reached is much more dominant than strain rate hardening, making that the extrapolation in strain rate is not relevant. The second is that, due as well to this high temperature softening, the yield strength of the materials suddenly drops. This makes that the deviatoric component of the stress is limited by yielding while nothing prevents the hydrostatic component to grow. The consequence of this is that the response is more governed by the EOS than by the strength model, making that the extrapolations and inaccuracies of the latter are not that important.

Tantalum response is without doubt the best within all the materials tested, presenting absence of macroscopic internal cracks even after irradiated at the highest intensity. This is confirmed until now by the online recorded non-distorted radial wave and current PIEs, consisting in visual inspections and Ultrasound Tests. This fact makes tantalum the strongest candidate material for the future AD-Target design. The absence of cracks is attributed to the low yield strength of tantalum and high elongation to break, which cause energy release through plastic yielding rather than crack formation. As a matter of fact, simulations crosschecks shown that the tantalum target was experiencing plastic deformation in a great part of its volume even from the lowest irradiation intensities. More detailed foreseen PIEs after slicing the irradiated targets such as optical metallography and SEM will bring important insights on the microscopic effects of this yielding, as well as revealing the presence of possible microscopic cracks. The study of the effect of this yielding in tantalum and its potential hardening effect is therefore one of the main interest of future works, since this will dictate the long term performance of this material (together with radiation damage, but it seems Ta has a relatively good response in that sense, as described in section 1.5.4 of Chapter 1). In any case, tantalum targets experienced relatively large plastic deformation during the experiment (it was impacted 13 times) if one considers that this was taking place already from the lowest intensity pulses. Even if in the long term its crack resistance properties can be jeopardized, it can be claimed that tantalum response is in any case better than the rest of materials, which fracture from the first impacted pulse. Future HiRadMat experiments will be devoted to study tantalum behavior under the impact of a larger number of pulses at high intensities to check this effect of yielding. Different geometries to try to minimize the effect of the radial wave and tensile pressures reached will be also tested. 
Of course, there is always room for improvements and things that could have gone better during the experiment after the acquired experience. For example, the adiabatic rise of temperature at targets surface was hardly measured by the pyrometer even if the acquisition rate was supposed to be enough. One of the reasons for that was the high minimum measuring threshold of temperature $\left(300{ }^{\circ} \mathrm{C}\right)$ of the pyrometer. This was set in such as way because, during the design phase, the AD-Target conditions (highest intensity pulses) were used as references for the instrumentation selection. At these conditions, the expected surface temperatures were above $1000{ }^{\circ} \mathrm{C}$ and for being able to measure this temperature the lower range had to be $300{ }^{\circ} \mathrm{C}$. However, later on during the experiment many of the impacted pulses were at lower intensities, since by the time high intensity pulses were impacted most of the targets were already extensively fragmented or bended, reducing the beam-target interaction and not reaching these high temperatures. Another problem associated to the pyrometer and still not fully explained is a recorded huge oscillation in the pyrometer signal observed during first microseconds, which is attributed to prompt radiation.

Another effect not foreseen during the design of the experiment was the extensive bending of the targets taking place due to the excited bending mode. As demonstrated by numerical simulations, this occurred even if the achieved offsets of the beam impacts were very low, even from a few tens of millimeters and less than $5 \%$ of the rods diameters. Nevertheless, this bending deformation is also a very useful lesson learned for the AD-Target core design or future HiRadMat experiments. A form of avoiding this effect could be just to reduce the length of the target by slicing it in independent smaller rods. In a similar way, the observed fragmentation of some of the graphite clamps may tell that this material is not the most suitable for the AD-Target containing matrix and that maybe another material, which is more flexible and could absorb and damp the incoming pressure waves from the target core, could avoid this problem. The preliminary concepts for the new AD-Target core configuration shown in Chapter 7 follow this lessons learned, proposing a target core composed by several small rods of different diameters embedded in a layered flexible expanded graphite (EG) matrix. 


\section{Chapter 7}

\section{Conceptual Design of the New AD-Target}

This last Chapter is devoted to the application of the acquired knowledge in this thesis for proposing a new optimized design of the AD-target. It must be mentioned at this point that the present proposal is a conceptual design and not necessarily the final one, since there are some aspects, such as details of the final core configuration, which have to be defined yet. Nevertheless, the strategies to follow are very well set.

As stated in the introduction, the design of the new AD-Target has to fulfill certain requirements in order to optimize the antiproton production. In addition, it has to provide a safe and robust operation, taking into account problems that have appeared during the past operation. These requirements can be summarized in:

- For optimizing antiproton production, the amount of material (and its density) surrounding the target core has to be minimized in order to reduce antiproton re-absorption. In addition, the structural stability of the target core may play a fundamental role, since its internal fracture would lead to a reduction of its effective density and beam-material interaction. Simulations and experiments performed in this thesis have shown that most probably the current iridium core is fragmented from the very beginning of operation. The new target core configuration would aim at avoiding this fragmentation by changes in the material and geometry.

- In addition to the fracture of the target core, simulations presented in Chapter 3 have shown that the graphite matrix is also subjected to very high dynamic stresses coming from the radial wave generated in the core. These 
stresses are well above the strength of graphite and therefore this matrix may be also damaged close to the core interface. The damage can have important detrimental effects such as improper positioning of the target core (which may be also fragmented) or loss of thermal contact between the core and the matrix. Therefore, it would be desirable that in the new design the matrix is made by a low density material that can withstand the pressure wave coming from the target core.

- Finally, the new design has to guarantee an efficient heat removal of the energy deposited inside the target. In the old design this is done by a water cooling system, while in the new one it is proposed to be substituted by a pressurized air system. The latter removes the problem of water activation and complications of its treatment (currently a station for controlling purity and radio contamination of the cooling water has to be present). Furthermore, cooling by air reduces drastically the risks and concerns of leaks in the circuit, making the system much more robust.

The next sections include the proposal of a new geometry and material configuration of the AD-Target, which aims at fulfilling the mentioned requirements. The information is structured in 3 main sections: (i) First, the new configuration of the target core and containing matrix is presented, including first prototyping activities. (ii) Followed by the proposal and CFD simulations of the pressurized air cooling system and (iii) a structural analysis of the target windows subjected to the thermal loads from the proton beam is presented, including a detailed fatigue analysis. Conclusions summarizing the main outcomes and the relevance of the present thesis are finally withdrawn to enclose the chapter and the thesis. 


\subsection{Proposed Core Configuration and Containing Matrix}

Figure 7.1 shows a proposal of the new configuration of the AD-Target core and containing matrix. External dimensions of the matrix are kept the same as in the old design (15 mm external diameter). This matrix will be inserted in the titanium body as shown in the section 7.2. On the other hand, three main characteristics differ significantly from the old design:

\subsubsection{New core material: Tantalum}

Baseline core material in the new design is tantalum instead of iridium. This decision is taken based on the results of the HRMT-27 experiment presented in Chapters 5 and 6 , which indicated that the high ductility of tantalum could prevent its internal fracture when subjected to the tensile pressure wave generated as a consequence of each proton beam impact or, at least, have an improved behavior in comparison to the other investigated materials. As it was showed in Chapter 6, iridium and the rest of high density materials tested in the experiment presented extensive cracking even from impacted intensities several times lower than the ADTarget conditions, while tantalum targets only presented ductile deformation. It is believed that potential absence of cracks in the tantalum core will avoid the drop of antiproton production historically observed at the beginning of operation when using iridium (now attributed to loss of effective density due to internal cracking).

The lower density of tantalum $\left(16.7 \mathrm{gr} / \mathrm{cm}^{3}\right)$ compared to iridium $\left(22.56 \mathrm{gr} / \mathrm{cm}^{3}\right)$ is compensated by an increased length of the target core (76 mm instead of $55 \mathrm{~mm}$ ) which will provide an equivalent interaction length. The detriment caused for being farther from the ideal situation in which the target is a punctual source (due to the a longer target core) is expected to be partially compensated by optimizing target/horn distance and the reduction of volume and density of the surrounding target material.

\subsubsection{New core geometry configuration}

As can be seen in the figure 7.1, the geometry of the target core is significantly different. The target core is sliced in small rods with diameter variation, being enlarged in every slice in comparison to the old design (up to $8 \mathrm{~mm}$ diameter in the central slices compared to the previous $3 \mathrm{~mm}$ diameter). The motivation of this increase of diameter is coming from the lessons learned in section 3.4 of Chapter 3 of this thesis, in which it was shown by hydrocode simulations that the destructive compressive-to-tensile radial wave present in the target core is generated as a consequence of a constructive interaction between the end-of-pulse tensile wave and the natural radial wave. This constructive interaction can be mitigated by 


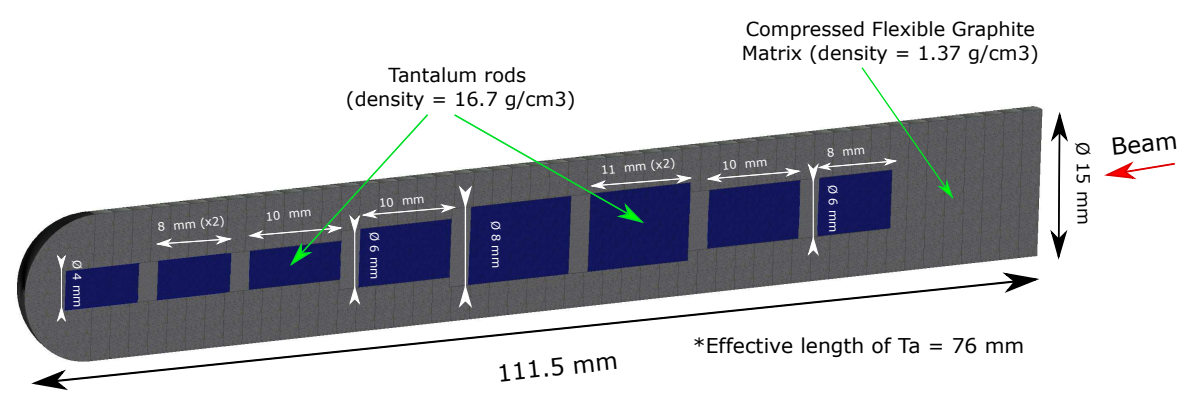

Figure 7.1: Half view of a proposed configuration of the new AD-target core, consisting in different Ta rods with different diameters embedded in a flexible Expanded Graphite (EG) matrix.

increasing the target diameter and therefore reducing the radial wave frequency. The target diameter variation in the different slices is selected consistently with the longitudinal profile of energy deposition, being larger in the areas in which higher energy density is deposited (higher magnitude of tensile pressures) and being reduced in the slices towards the downstream window. This decrease of diameter closer to the downstream window is done in order to reduce the antiproton re-absorption in the core periphery. This was the main motivation to have a target core as thin as possible in the old design, however, for the new design it is considered that the antriproton re-absorption contribution of the periphery material in central slices (with larger diameter) is very low since most of the antiprotons created there are scattered towards the downstream with very low angles.

The slicing of the target core in smaller and shorter rods is also motivated by the targets bending observations during HRMT27 experiment (section 6.3 in Chapter 6 ). It is clear that shorter rods will reduce this effect originated from excitation of bending modes due to beam impact offsets.

It is important to remark that the geometrical dimensions presented in figure 7.1 are still a first proposal which has to be validated by means of FLUKA simulations to confirm the positive impact in antiproton production, as well as by hydrocodes to check the generated pressure waves inside the slices. Geometry optimization can still take place, but in any case the concept and strategy is well defined. 


\subsubsection{Compressed Expanded Graphite (EG) as target core containing matrix}

Third proposed change has to do with the containing matrix material. In the old design this matrix was made by graphite, but hydrocode simulations shown in section 3.8 suggest that this matrix may be fractured due to the propagation of the radial wave from the target core. It is for this reason that this conventional graphite is replaced in the new design by a layered configuration of EG (commercially named as SIGRAFLEXß) ). Sheets of EG are manufactured from natural graphite flakes with well-ordered high-crystalline structure, fixed and subjected to high temperature thermal shocks in which the graphite flakes experience a huge unidirectional expansion, creating worm-like graphite flakes. Graphite worms are then compressed into foils and sheets without the use of any binder or filler [219].

Sheets of EG behave as a soft and flexible material (young modulus under compression in the transverse direction $0.5 \mathrm{GPa}$ ). One of the main commercial use of EG sheets is in seals and gaskets as a rubber substitute in high temperature applications [220]. Despite they can suffer from delamination when bended, this is not expected to take place if they are arranged and compressed in parallel. EG sheets present very high thermal conductivity in the in-plane direction $(250 \mathrm{~W} / \mathrm{mK})$ and very low density (around $1 \mathrm{gr} / \mathrm{cm}^{3}$ ) which can be adapted depending on the compression of the grade. Furthermore, it has very good stability at high temperatures, in particular in inert atmosphere, since it is just constituted by carbon. These characteristics make it very attractive as matrix material of the AD-Target. In addition, the flexibility and softness of the EG could be very beneficial for absorbing and damping the radial wave coming from the target core without breaking.

The layered matrix configuration proposed is shown in figure 7.1. The idea is to have several hollowed disks of EG, $3 \mathrm{~mm}$ thickness, surrounding the target core. These layers would be compressed during the assembly of the target applying pressures of the order of $30 \mathrm{MPa}$ (see next section 7.1.4). Radial expansions of the EG layers due to Poisson's effect will ensure a very good thermal contact between the target core and the matrix material. High in-plane thermal conductivity provides efficient heat removal towards the periphery. In addition, the pre-compressed state of the matrix could help for keeping the matrix-core contact along their interface when receiving the radial waves and against eventual plastic deformation of the tantalum core. Another important aspect in the EG matrix configuration proposed in figure 7.1 is the presence of single EG disks between the slices of the tantalum core. These EG disks are independent from the external hollowed disks and play a very important role during the compression of the assembly since they compensate the incomprehensibility of the Ta core with respected the latter. In addition, these inner EG disks could reduce wave transmission between the different core slices.

Few aspects have to be investigated to confirm the viability of the proposed EG configuration for the AD-Target matrix: 
I Level of compressibility of the layered EG matrix in terms of necessary pressure and deformation for obtaining substantial Poisson's effect to guarantee good thermal contact between the matrix and the core.

II Validate an assembly procedure for obtaining the compressed EG matrix around the target core and encapsulation in the titanium envelope by electron-beam welding.

III Behavior of the target core-matrix interface during operation when successive radial waves will be transferred to the matrix. The key aspect to understand is if the matrix interface could be damaged or suffer plastic deformation to the extent in which it is not able to recover the Ta surface shape and therefore, leading to the appearance of gaps. Note that the radial waves have compression and tensile phases and in the interface the deformations produced during the tensile period could be different in both materials, forming such gaps or small holes.

The high anisotropy of the EG material and layered configuration makes hard to predict accurately its behavior by simulations. Furthermore, other uncertainties such as porosity or possible air tapered in the layers complicates the prediction of deformation and reaction forces at compression phase during assembling. In addition, it has not been found in the literature detailed information about the mechanical properties of EG (such as Poisson ratio) when compressed to a large extend, which can also differ significantly depending on the grade (density and thickness). It is for these reasons that an experimental and prototyping approach is being carried out to answer the three uncertainties just mentioned. 


\subsubsection{Prototyping Activities for Manufacturing an EG-Matrix Embedding the Target Core}

The adopted strategy and preliminary results for answering the three main concerns just mentioned are presented in this subsection:

\section{Mechanical Characterization of EG}

First, different mechanical tests have been performed for answering the level of compressibility necessary for a sufficient radial expansion of the EG by Poisson effect. The goal of these tests was to obtain macroscopic information of the material response which could be directly applicable to the prototyping activity, rather than a detailed characterization of the intrinsic material properties, which would require a much deeper analysis due to the complex internal structure of the material. In this context, three main types of tests have being performed: (i) Uni-axial tension in-plane direction. (ii) Uni-axial compression in the perpendicular plane. (iii) Radial expansion of a single disk during compression.

Figure 7.2 shows the true strain-stress curve of a single sheet, $2.5 \mathrm{~mm}$ thickness, of EG graphite subjected to tension in its in-plane direction. Geometry of used specimens is shown in the right hand side of the figure. As it can be observed, EG presents a very soft and weak response, with a young modulus in tension of only $530 \mathrm{MPa}$ and with very large deformation to break. It starts presenting yielding below $1 \mathrm{MPa}$ of stress.
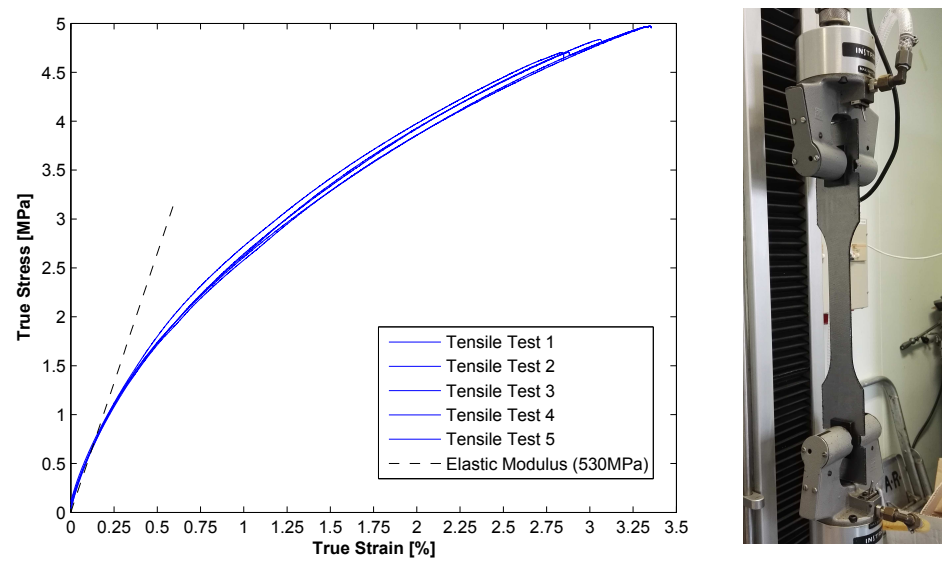

Figure 7.2: Tensile true strain-stress curve of a single sheet of EG of -2.5 mm thicknesstested on its plane direction (see picture in the right). 


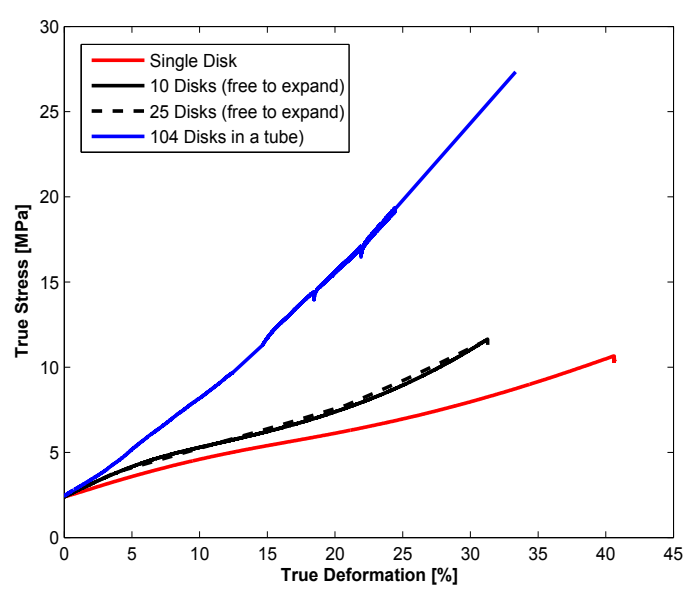

(a)

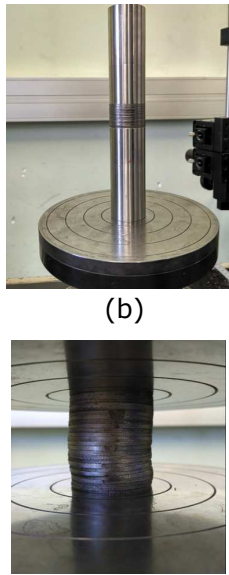

(c)

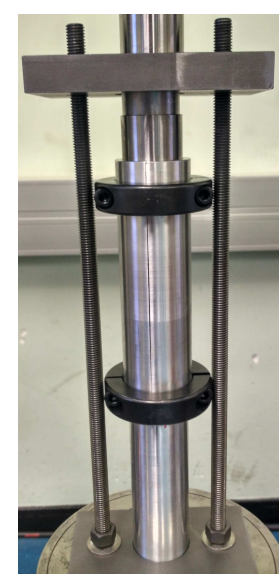

(d)

Figure 7.3: (a) True compressive strain-stress curves in the perpendicular plane direction of EG disks. Four different configurations are presented; single disk of $40 \mathrm{~mm}$ diameter free to expand, stacks of 10 and 25 disks free to expand (pictures (b) and (c)) and (d) a stack of $40 \mathrm{~mm}$ diameter hollowed disks embedded in a tube equivalent to the prototype geometry of figure 7.5 .

In a similar way, different compressive tests of EG in the perpendicular plane direction have been performed. These tests are particularly interesting since in the proposed target matrix configuration the EG layers will be compressed in this way. Relevant information such as the level of achievable deformation and necessary forces during assembly can be extracted from these tests. Specimens in form of disks of $40 \mathrm{~mm}$ diameter were used, sharing geometry with the scaled prototype of the target presented in the figure 7.5 of the next section. Necessary forces and deformation ratios are not necessarily the same when compressing a single disk (free to expand radially), a stack up of disks (free to expand), or a stack of disks embedded in a tube. These three configurations have been compressed and results are presented in figure 7.3. The obtained strain-stress curves are plotted in the plastic region, after an initial pre-stress of 2.4 MPa. Important differences in the stiffness of the response between the configuration tested can be observed. These differences are attributed to the interface behavior between layers or other effects such as trapped air in the porous EG material. A stack of 10 or 25 disks (figures 7.3(b)(c)), for example, presents more resistance to compressive deformation than a single disk. In that sense, the most interesting curve for our purpose is the one corresponding to the in-tube compression which picture is shown in figure 7.3-(d). This configuration is an equivalent geometry of the proposed target and prototype of figure 7.5, including the hollowed disks and central metal rods representing the target core. Obtained forces for compression of this configuration are therefore directly applicable to the scaled prototype presented in the next section. As can 
be seen in the blue curve of figure 7.3-(a), a deformation of $30 \%$ can be achieved by a pressure of $24 \mathrm{MPa}$, equivalent to $30 \mathrm{kN}$ (3 tons) of force for the proposed prototype geometry.
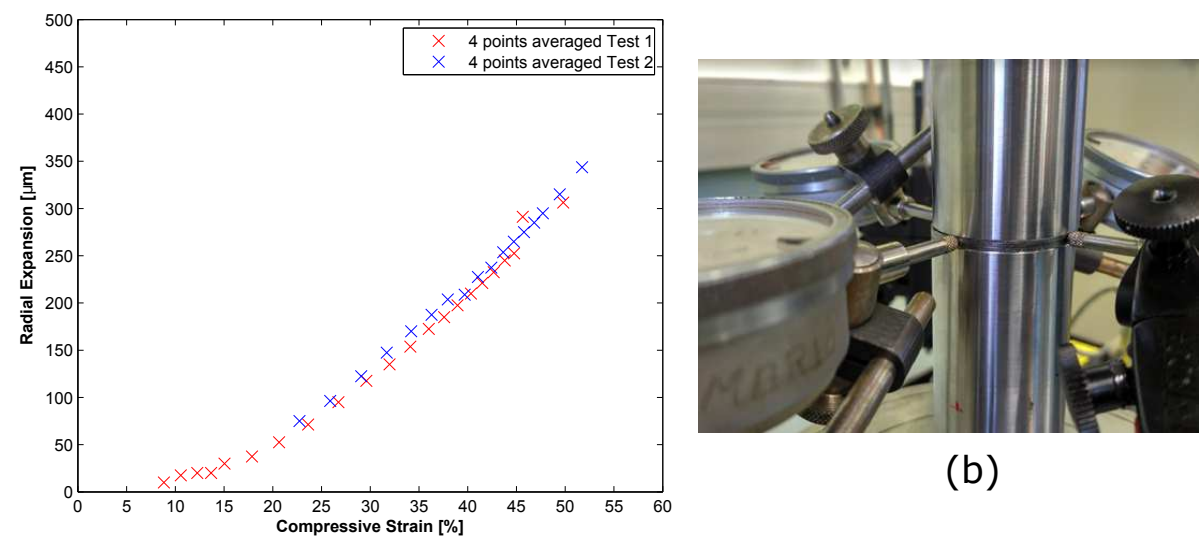

(b)

(a)

Figure 7.4: (a) Measured radial expansion due to Poisson's effect of a single EG disk submitted to compression. (b) Picture of the used experimental setup based on 4 dial calipers.

Finally, in figure 7.4-(a) an estimation of the radial expansion due to Poisson's effect of a $40 \mathrm{~mm}$ diameter disk of EG is shown. This plot has been obtained by compressing a single disk (free to expand radially) while measuring its change in diameter by four dial calipers as show in figure 7.4-(b). By putting together this plot with the level of in-tube achievable compression shown in figure 7.3 , it could be claimed that with a compressive deformation of $30 \%$ obtained by an in-tube compression of $24 \mathrm{MPa}$, radial expansions of 150-250 $\mu \mathrm{m}$ could be achievable. This radial expansion should be enough to fill possible gaps due to EG and Titube casing tolerances and for guaranteeing a radial pressure contact of a few MPa between the target core and containing matrix. 


\section{Assembling of a scaled Prototype and Irradiation in HiRadMat}

Using the information of mechanical tests provided in the previous subsection, a first assembly of a scaled prototype of target core and EG containing matrix is built. The reason for scaling is to be able to irradiate directly the assembled prototype in HiRadMat. As it was described in Chapter 4, a scaling of the dimensions in AD-Target is necessary for recreating equivalent conditions in HiRadMat facility due to the much higher proton beam energy of the latter $(440 \mathrm{GeV}$ instead of $26 \mathrm{GeV}$ ), which, as a consequence, requires beam defocusing and scaling up of the geometry.

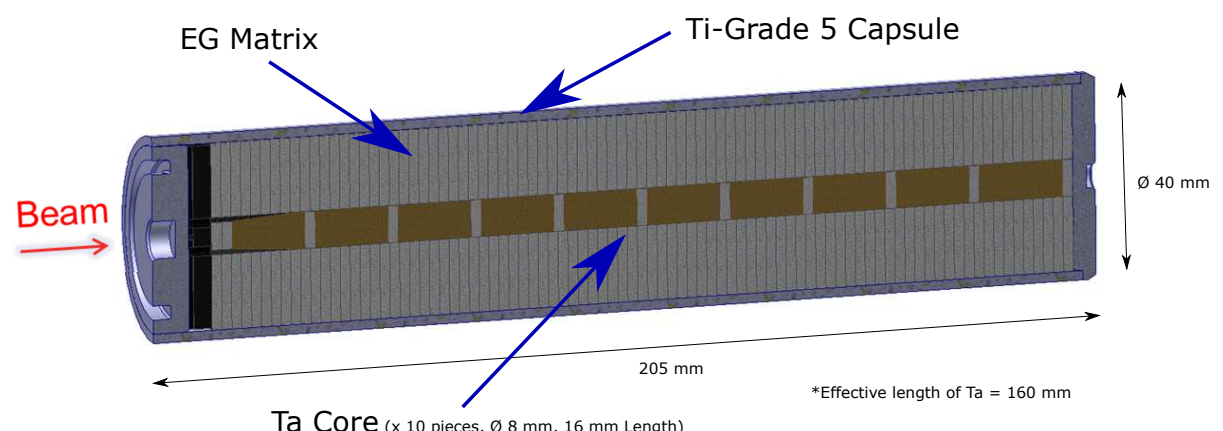

Figure 7.5: Half-cut representation of the built scaled prototype of target, containing a sliced $8 \mathrm{~mm}$ diameter Ta core embedded in a EG-matrix and Ti-6Al-4V capsule.

Figure 7.5 shows a representation of the first scaled prototype of the EG-embedded tantalum core, which will be irradiated in HiRadMat by a beam with the same characteristics as the one used to recreate AD-Target conditions in HRMT27. The target core consists in ten small rods of pure Ta, $8 \mathrm{~mm}$ diameter, with a total effective length of $160 \mathrm{~mm}$, being therefore the same effective geometry as in HRMT27. This experiment has been named HRMT42-TaScat.

In this case, differently from HRMT27, the target core is embedded in a $40 \mathrm{~mm}$ diameter matrix consisting in hollowed EG disks. This matrix is compressed inside a $2 \mathrm{~mm}$ thickness Ti grade 5 alloy tube, and closed by two lids which are electronbeam welded. The total length of the assembly is $205 \mathrm{~mm}$. The achieved level of compression of the EG matrix is in the order of $30 \%$. The use of the same $8 \mathrm{~mm}$ diameter for the Ta target core as in HRMT27 will allow a direct comparison of the response of that single irradiated target with respect the sliced and embedded rods in the present assembly. In this way, one could expect to draw conclusions of the beneficial effects of sliding the target (such as reducing bending) and, at the same time, re-validate the good response of tantalum when impacted by a higher number of high intensity pulses than in HRMT27. This does not prevent in any case the intention of using several sliced rods with different and larger diameters in the new AD-Target design as introduced in figure 7.1. However, it was considered 
more convenient to start with a simpler prototype in which the diameter of all the sliced rods are the same, and adding gradually more complexity in future prototypes.

Figure 7.6-(a) shows the stacks of $40 \mathrm{~mm}$ diameter EG hollowed disks and small $8 \mathrm{~mm}$ diameter metal rods prior to assembly and compression in an aluminum tube. This Al-tube assembly corresponds to the one previously presented in the picture of figure 7.3-(d) and which strain-stress response during compression it was shown in figure 7.3-(a). The objective of this first assembly was to quantify the mentioned response and to validate the mounting procedure consisting of using two half cylinders for guiding of the EG-disks during compression. Figure 7.6-(b) shows a X-ray image of the compressed assembly in which the different sliced rods of the target core can be observed. Foils of $0.1 \mathrm{~mm}$ thickness of copper were also added between some of the EG layers in order to track the uniformity of the achieved compression, since the low density EG is transparent to the X-rays. By using this technique it was possible to observe that undesired interactions between the core rods and EG hollowed disks did not take place, as well as that compressions ratios ranging from $35 \%$ (in the top side) to $21.6 \%$ (in the bottom) were achieved. This non-uniform compression is attributed to the friction of the EG disks with the external tube, due to the radial expansion by Poisson effect. It was concluded any case that this non-uniform is unavoidable and acceptable.

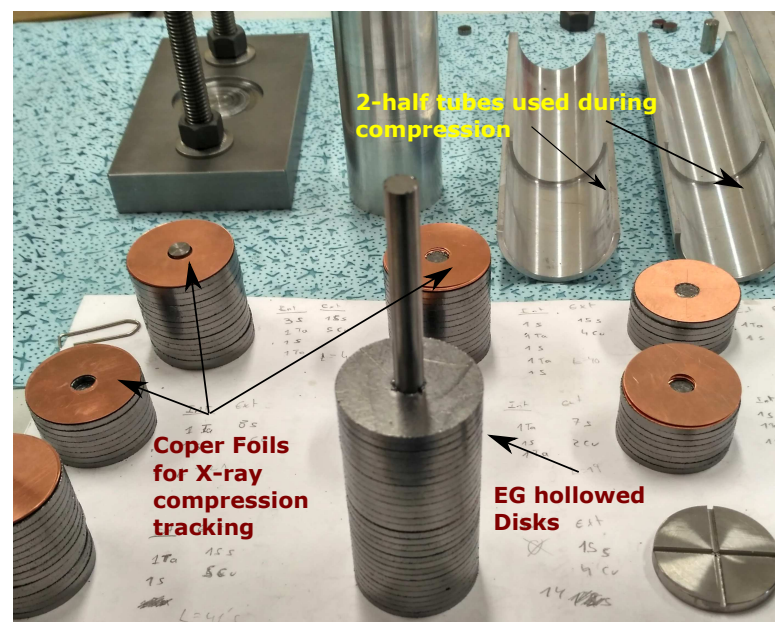

(a)

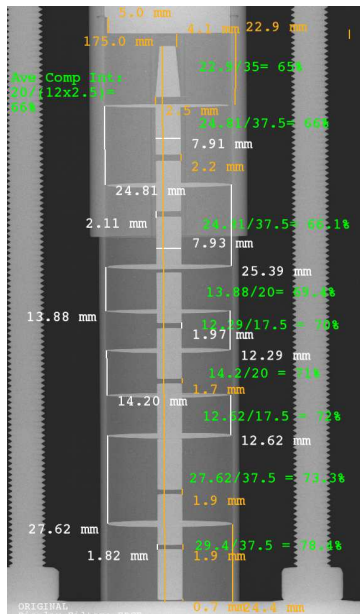

(b)

Figure 7.6: (a) Picture of the stacks of EG hollowed disks, core, and copper foils prior to assembling and compression in $\mathrm{Al}$ tube, together with two half cylinders used for guiding the EG during compression. Compression curve and setup has been already shown in figure 7.3-(a)(d). (b) X-Ray image of the compressed setup used for verifying the achieved compression ratios. 


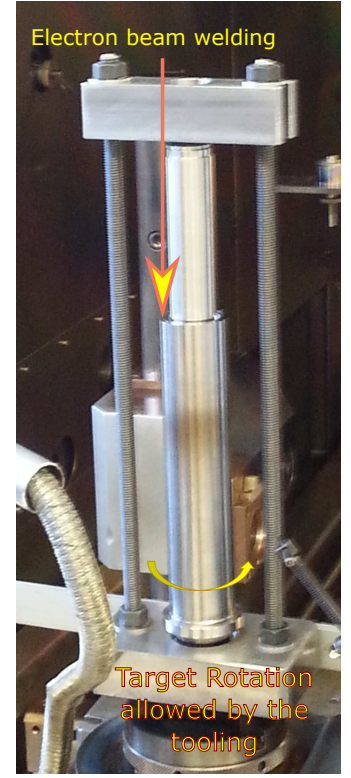

(a)

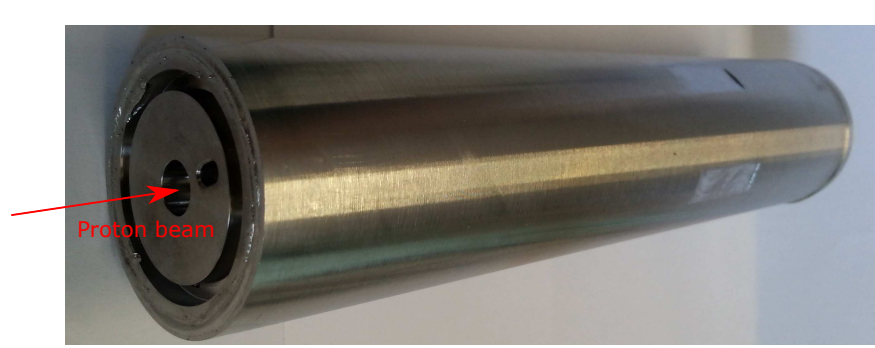

(b)

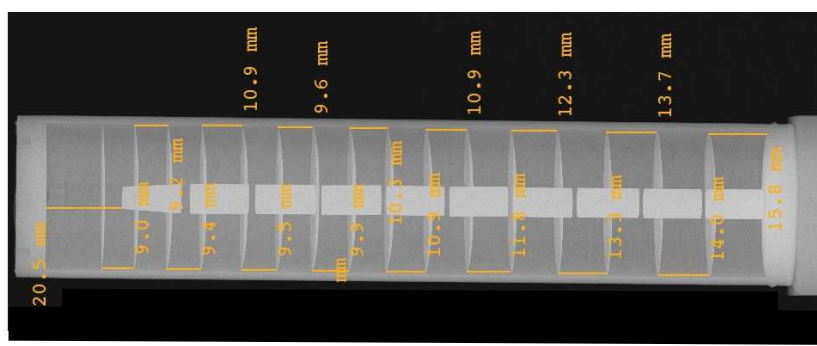

(c)

Figure 7.7: (a) Picture of the scaled prototype in its dedicated compression tooling prior to e-beam welding. (b) Picture of closed target after welding. (c) X-ray image of the interior of the target.

Once the assembling process was validated, a first prototype was built, using in this case a Ti-6Al-4V tube with one of the ends closed with a lid which was previously e-beam welded. Figure 7.7-(b) shows the picture of the assembled scaled prototype. E-beam welding of the upper lid while maintaining the EGmatrix compressed was done with the aid of the tooling shown in figure 7.7-(a). This tooling was equipped with radial and axial bearings which allowed rotation of the target with respect to its axis inside the vacuum chamber of the machine. In this way, the electron beam (coming from the top in the picture) could go over the lid-tube radial interface and close the capsule. The tool maintained the EG matrix at a pressure of $25 \mathrm{MPa}$ while welding. Figure 7.7-(c) shows a X-ray image of the closed target, in which the sliced Ta target can be observed. Foils of $\mathrm{Cu}$ were also added between some of the EG-layers so they compression ratio can be evaluated. An overall compression of $27 \%$ in the EG was achieved, although it is possible to observe that the compression was not really uniform. At the upstream parts higher levels of compression were reached $(40 \%)$ while in the downstream only $10 \%$. Again, this is due to the high friction between the EG and the Ti tube at the interface while compressing from the upstream side due to the radial EG expansions. Less tight tolerances in the radial adjustment between the EG matrix 
and Ti tube will be used in the future to overcome this issue, as well as performing the compression in different steps.

This prototype was irradiated in HiRadMat in May 2017. Figure 7.8 shows the target assembled in the experimental sample holder. The experimental setup is re-used from the HRMT27 experiment, with a different sample holder. The irradiation of this prototyped target is done sharing experimental setup with $\mathrm{Si}$ crystals (shown at the top of the picture), which will be also irradiated in the context of another project related to crystal collimation. Prior to irradiation, a 3 -D tomography of the target using synchrotron radiation has been performed at the European Synchrotron Radiation Facility (ESRF) in Grenoble. Images of the Ta-EG interface with $15 \mu \mathrm{m}$ resolution has been obtained with this technique. After irradiation in HiRadMat, another tomography of the target is foreseen in order to look for possible changes, gaps, or detachments in the interface due to plastic deformation in the Ta core or displacement of the EG matrix due to the radial wave.

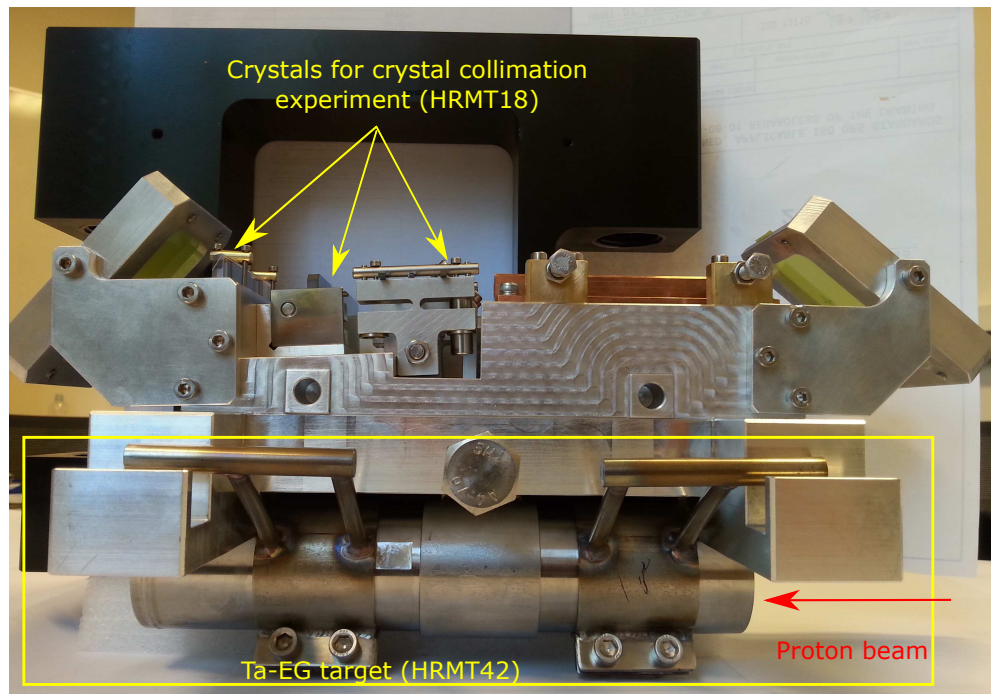

Figure 7.8: (a) Ta-EG target assembled in the sample holder prior to installation and irradiation in HiRadMat (named HRMT42 experiment). The target shares experimental setup with 3 crystals that were also irradiated in the context of crystal collimation R\&D (HRMT18-CRY2 experiment). 


\subsection{Proposed Pressurized Air Cooling System}

\subsubsection{Proposed Geometry}

In addition to the new configuration of the target core and containing matrix, a new external target geometry is proposed. The purpose of this new geometry is to adapt the target to a pressurized air cooling system (instead of the water system of the past design) and to optimize the amount of mass surrounding the target material. Figure 7.9 shows a representation of the new proposed external geometry, which consists of two independent casings made of Ti-6Al-4V. Inside the internal casing the target containing matrix and target core already shown in figure 7.1 would be fitted by compression following a similar procedure as the one of the prototype presented in the previous section. The pressurized cooling air would flow between the two Ti-alloy casings, following a serpentine machined externally in the internal one. The channel of the serpentine has a cross section of $3 \times 2 \mathrm{~mm}$. Assembling of the inner and outer casing could be done by shrink-fitting in order to provide a very good thermal contact.

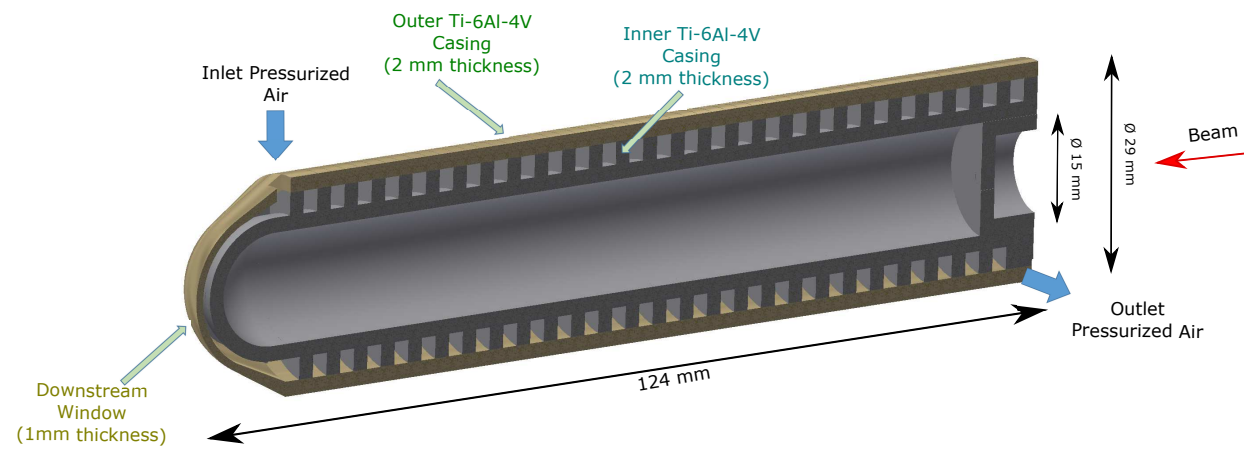

Figure 7.9: (a) Half-view representation of the new proposed geometry of the ADTarget envelope, consisting in a double Ti-6Al-4V casing with an internal serpentine circulated by pressurized air.

The inlet of the cooling system would be close to the downstream window of the target, cooling it specifically by flowing through a gap of $1 \mathrm{~mm}$ between the inner and outer casings. This extra cooling of the downstream window is included in order to reduce temperatures and thermal stresses there, since this is the most critical part of the assembly and could suffer from fatigue during long term operation. The double wall downstream window serves also as an extra containment to radionuclide release in case of internal casing failure.

Next subsections include CFD and thermo-mechanical analyzes of the new proposed geometry during operation, as well as dimensioning of the air cooling flow parameters. 


\subsubsection{CFD Simulations and Cooling Flow Parameters}

First step of the validation of the new proposed geometry consists in the definition of flow parameters necessary for cooling the target. For this purpose, CFD calculations have been performed using ANSYS-CFX®. This computational model will allow to estimate the amount of heat that can be removed from the target by the convection of the cooling flow. A conjugate heat-transfer model including both solid and fluid domains is therefore implemented. The objective of the simulation is to obtain the convective heat transfer coefficient (HTC) - $h$ - along the serpentine walls for the considered flow parameters (pressure and mass flow rate), as well as to estimate the increase of temperature in the cooling fluid and potential pressure drop along the serpentine.

In order to simplify the CFD model, only steady state simulations have been performed. These simulations are therefore considering that the power is constantly deposited in the target core by the beam. This consideration is supported by the assumption that, even if in reality the heat is deposited in a very discontinuous manner in the core, the time necessary for heat diffusion from the core to the serpentine will homogenize in time the reached temperatures close to serpentine, being closer to a steady state solution, especially from the point of view of the fluid. This assumption is also particularly valid if ones takes into account that the main outcomes of this simulation are the flow parameters (fluid velocities/pressure/temperatures and HTC at the serpentine surface). This HTC $-h-$ will be applied later on to the ANSYS mechanical transient thermal model shown in section 7.2.3, which does take into account the fast deposition of energy in the target core and its diffusion towards the serpentine.

The pressurized air flow is considered as an ideal gas in the simulations. Pressure inside the air flow serpentine is considered 20 bars, which would be delivered by a commercial compressor. In reality, the circulating flow will be in an open circuit with the exhaust inside the AD-Target hall. In this way, this potentially activated air at the exhaust will be treated by the close ventilation system already present in the area which, for safety reason, keeps the area with a slight negative pressure relative to the atmospheric pressure. For this reason, maximum allowed cooling flow for avoiding overcharging the area ventilation system is set at $60 \mathrm{Nm}^{3} / \mathrm{h}$. Given these input parameters. The boundary conditions of the CFD model are:

I Pressure of 20 bars and room temperature at the inlet (it is considered that a commercial cooling and air-dryer stage is present after the compressor).

II A mass flow rate of $1.8 \mathrm{gr} / \mathrm{s}$ is set in the outlet, equivalent to a volumetric flow at normal conditions of $5.47 \mathrm{Nm}^{3} / \mathrm{h}$ (well below the maximum allowed value just mentioned). 
III The energy deposited by the beam is applied in form of internally generated power in the solid domain of the model by a tabular spacial-load. This tabular load interpolates the deposited energy obtained by FLUKA simulations to the mesh of the CFD model. For this simulation it is assumed that the core is made of iridium, since this is the most conservative case due to its high density and deposited energy, even if the baseline target core configuration in the new design is tantalum. The total energy deposited per pulse is $2480 \mathrm{~J}$. Since it is a steady state simulation, the deposited energy is averaged over $9.6 \mathrm{~s}$ which correspond to the minimum time between consecutive pulses in the most conservative future operation. This leads to a total power of $260 \mathrm{~W}$ which has to be removed by the cooling flow.

A Shear-Stress-Transport (SST) turbulence model [221] is used in the fluid domain. This model combines the k-omega turbulence model and k-epsilon turbulence model such that the k-omega is used in the inner region of the boundary layer (so effects like convection and diffusion of turbulent energy are taken into account) and switches to the k-epsilon in the free shear flow.

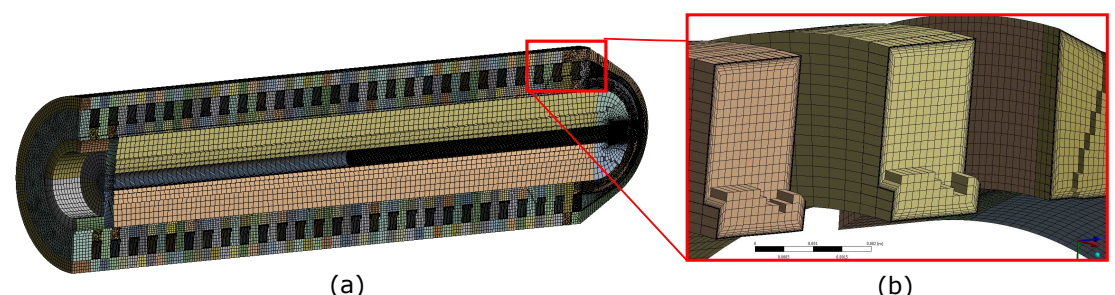

(a)

(b)

Figure 7.10: (a) Image of the mesh used in the conjugate heat transfer CFD calculations, showing the solid and fluid domain. (b) Zoom-in of the mesh in the fluid domain, showing the inflation layers close to the solid domain interface.

Figure 7.10(a) shows the mesh of the CFD model which contains 6048595 elements. 12 inflation layers are included close to fluid-solid interface as shown in figure $7.10(\mathrm{~b})$, with a first layer size of only $1 \mu \mathrm{m}$ in order to simulate accurately the flow at the boundary layer and therefore the convective heat transfer. With this inflation layer, a maximum $y+$ factor of 0.8 is achieved in the cooling channel. Maximum mesh skewness factor is 0.55 , while most of the elements stay on 0.05 . Minimum orthogonal quality is 0.64 while most of the elements stay on 0.94 .

Figure 7.11(a-d) shows respectively the velocity, pressure, temperature and achieved HTC along the cooling channel. Table 7.1 summarizes the inlet and outlet parameters according to the CFD results. Mean velocity of the fluid is around $15 \mathrm{~m} / \mathrm{s}$, leading to a pressure drop in the circuit of around 1 bar. Increase of temperature of the fluid along the channel is around $75{ }^{\circ} \mathrm{C}$, which is considered acceptable for the exhaust in the AD-Target hall. The HTC shown in figure 7.11(d) has been 
estimated by a post-processing script which takes the calculated heat flux at each point on the channel's surface and divides it by the $\Delta \mathrm{T}$ between the same point on the surface and the bulk fluid, taken from an offset point at center of the channel. These HTC and fluid bulk temperatures are extrapolated and applied in tabular

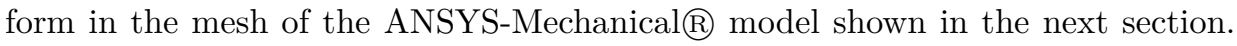
It can be seen that with the given fluid parameters a HTC in the range between 1000 and $1500 \mathrm{~W} / \mathrm{m}^{2} \mathrm{~K}$ is achieved.
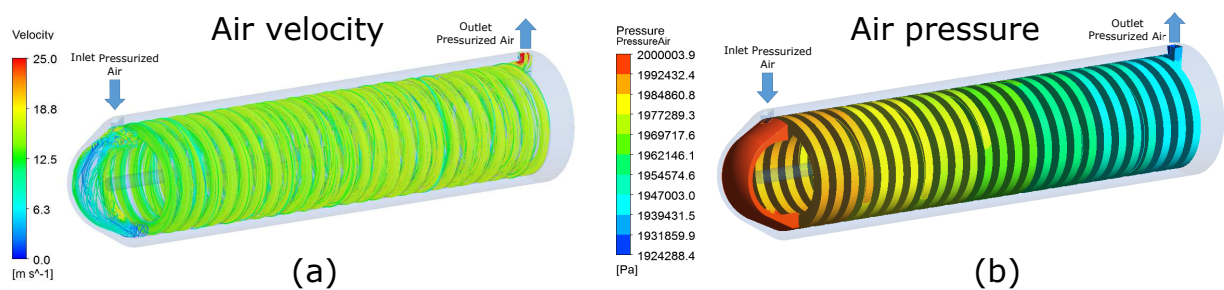

(b)
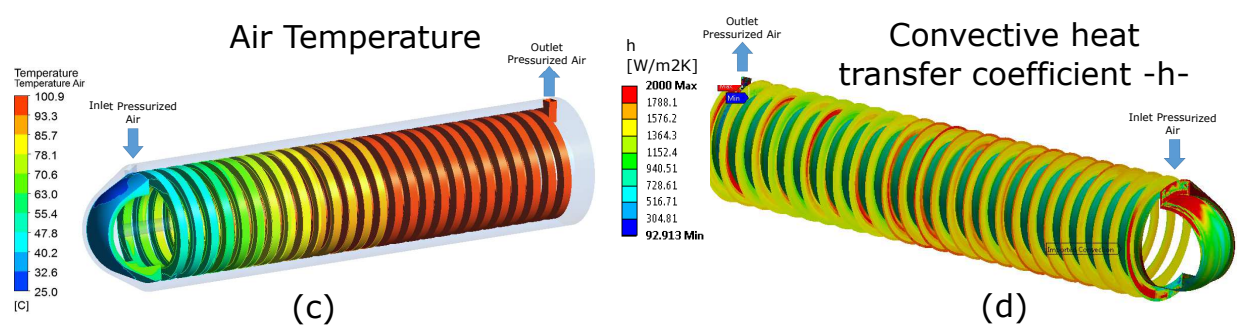

Figure 7.11: Contour plots of the air flow along the cooling serpentine: (a) Fluid velocity, (b) Fluid pressure, (c) Fluid Temperature, (d) HTC at the serpentine's wall.

Table 7.1: Summary of air parameters in the new proposed AD-Target cooling system.

\begin{tabular}{ccc}
\hline Parameter & Inlet & Outlet \\
\hline Pressure $[\mathrm{bars}]$ & 20 & \multicolumn{2}{c}{19.2} \\
Mass Flow $[\mathrm{gr} / \mathrm{s}]$ & \multicolumn{2}{c}{1.8} \\
Volumetric Flow at Normal Conditions $\left[\mathrm{m}^{3} / \mathrm{h}\right]$ & \multicolumn{2}{c}{5.47} \\
Temperature $\left[{ }^{\circ} \mathrm{C}\right]$ & 25 & \multicolumn{2}{c}{100} \\
Mean Velocity $[\mathrm{m} / \mathrm{s}]$ & \multicolumn{2}{c}{15} \\
HTC achieved $\left[\mathrm{W} / \mathrm{m}^{2} \mathrm{~K}\right]$ & \multicolumn{2}{c}{$1000-1500$} \\
\hline
\end{tabular}




\subsubsection{Thermal Analysis of the Target Assembly}

After the CFD calculations of the cooling fluid, thermo-mechanical calculations using ANSYS-Mechanical@ have been performed in order to know accurately the temperatures reached in the Ti-6Al- $4 \mathrm{~V}$ envelope taking into account the transient deposition of energy in the target core. The HTC shown in figure 7.11 has been extrapolated and applied to the mesh of the serpentine's surface in the model, together with the fluid bulk temperature for each corresponding position. As usual, the energy deposited by the primary proton beam has been applied to the model by interpolation of results from FLUKA Monte Carlo Simulations. Iridium has been considered as target core material as this is the most conservative case in terms of deposited power, even if in the reference configuration the target core will be made of Tantalum. Thermal contact conductance (TCC) between the different parts such as core, containing EG matrix, and Ti-6Al-4V envelope is assumed to be perfect. This assumption, even if far from reality, is conservative in terms of temperatures and stresses reached on the Ti-6Al-4V envelope, which is the most critical part from the point of view of heat management, since the graphite matrix and target core made of a refractory metal will easily withstand the high reached temperatures.

The simulation has been carried out in two steps in order to estimate accurately the temperatures reached during the extended operation. First, a steady state simulation considering a constant power deposition equivalent to a proton pulse impact every $9.6 \mathrm{~s}$ has been performed. Then, a transient simulation starting from the steady state temperature profile and considering three proton pulse bursts is carried out. Figure 7.12 summarizes the obtained temperatures at different times and components.

Figure 7.12-(a) shows the temperature profile in the whole target assembly right before a proton pulse impact (which is the same as the steady state solution). It can be seen that during the $9.6 \mathrm{~s}$ of cooling between two consecutive pulses the temperature in the core drops down to $130{ }^{\circ} \mathrm{C}$. It can be observed also a longitudinal gradient of temperature due to the high anisotropy in thermal conductivity of the EG-matrix. Plot of figure 7.12-(b) shows the time-history of the maximum temperature reached in the hypothetical iridium core. The maximum reached temperature above $2200{ }^{\circ} \mathrm{C}$ is similar as in the past design. Nevertheless, this maximum temperature would be reduced down to $1700{ }^{\circ} \mathrm{C}$ if the final core target material in the new design is tantalum, due to its lower density.

Similarly as in figure 7.12-(a), figure 7.12-(c) shows the steady-state temperature profile in the Ti- $6 \mathrm{Al}-4 \mathrm{~V}$ envelope. It can be seen that the half volume of the envelope towards the upstream part stays at $100{ }^{\circ} \mathrm{C}$, while the downstream window is efficiently cooled down to $30^{\circ} \mathrm{C}$. Figure 7.12 -(d) shows the time history of the maximum temperature reached in the envelope taking into account the proton pulse impacts. A maximum reached temperature of $320{ }^{\circ} \mathrm{C}$ takes place in the 
upstream window, becoming also a critical part. A more detailed analysis of the temperature and stresses in the windows is included in the next section.

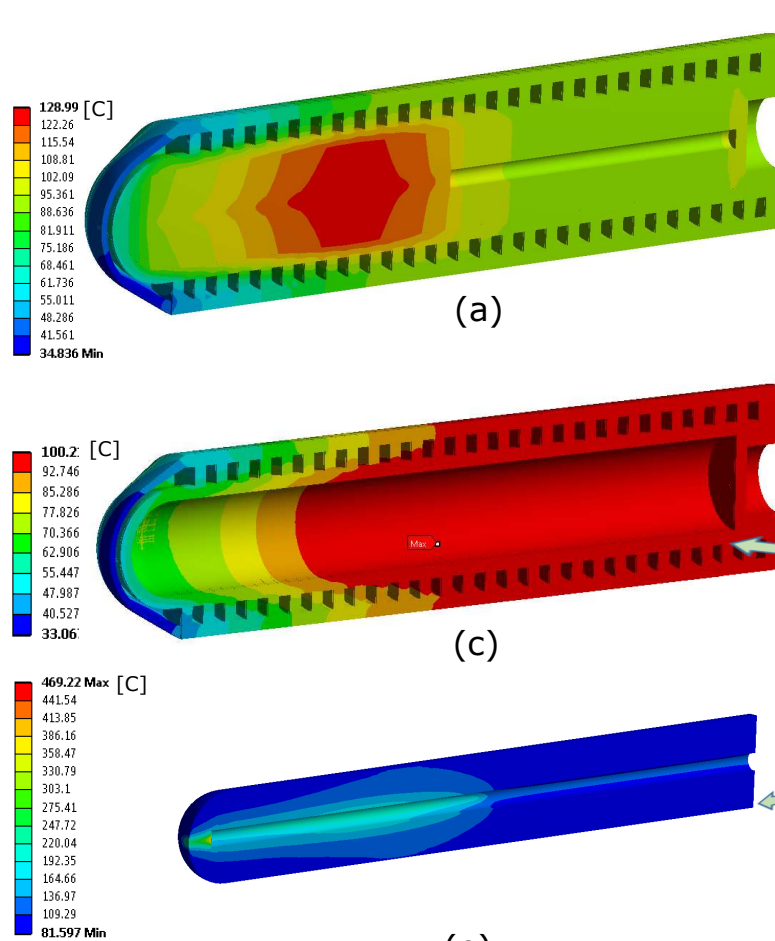

(e)

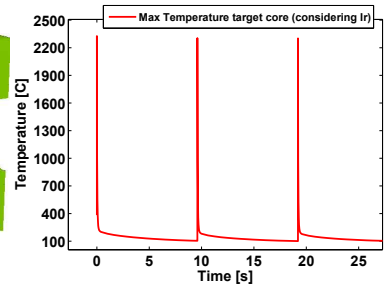

(b)

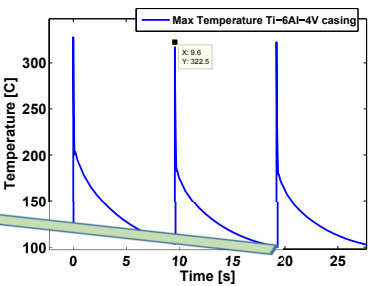

(d)

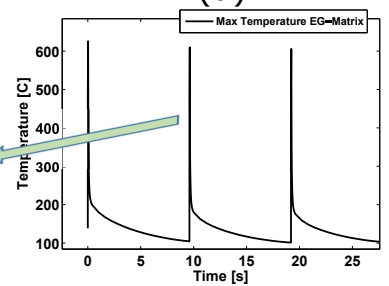

(f)

Figure 7.12: Summary of reached temperatures at different times and parts of the new proposed AD-Target. (a) Whole assembly at steady state. (b) Maximum temperature history in the core. (c) Ti-6Al-4V envelope at steady state. (d) Maximum temperature history in the Ti-6Al-4V envelope. (e) EG-matrix at the end of the pulse burst. (f) Maximum temperature history in the EG-matrix.

Finally, figures 7.12-(e) and (f) show the temperature reached in the EG-matrix. Figure 7.12-(e) shows the temperature profile right at the end of the proton beam impact. A maximum temperature of $470{ }^{\circ} \mathrm{C}$ is reached in the downstream part of the matrix. Figure 7.12-(f) plots the time history of temperature, showing that a maximum temperature of $620{ }^{\circ} \mathrm{C}$ is reached a few milliseconds after the pulse impact in some specific areas due to the heat diffusion from the target core, which is at much higher temperature. The bulk temperature of the EG-matrix drops down to $100{ }^{\circ} \mathrm{C}$ during the $9.6 \mathrm{~s}$ of cooling. 


\subsection{Structural and Fatigue Analysis of the Ti-6Al-4V Beam Windows}

As mentioned in the introduction of the present thesis, as well as pointed out in the previous section, windows of the target are the critical parts of the target envelope since the have to withstand the direct impact and energy deposition of the primary proton beam. These windows and, in particular, the downstream one, have to be properly cooled down and verified against stresses and fatigue. Indeed, overheating and thermal fatigue was identified as the source of a downstream window failure in an old rhenium target in the middle $80 \mathrm{~s}$, leading to contamination of the area [29].

Figure 7.13 shows the temperature profile at the end of the proton pulse burst and transient time history of the upstream and downstream windows. As can be seen the in the figure, maximum reached temperatures are $280{ }^{\circ} \mathrm{C}$ and $320{ }^{\circ} \mathrm{C}$ in the downstream and upstream windows respectively. There temperatures are reached in only $0.4 \mu \mathrm{s}$ similarly as it is taking place in the target core. The time history shows how these temperatures go down in a few seconds, in particular in the downstream window, which is more efficiently cooled as is close to the inlet of the pressurized air.
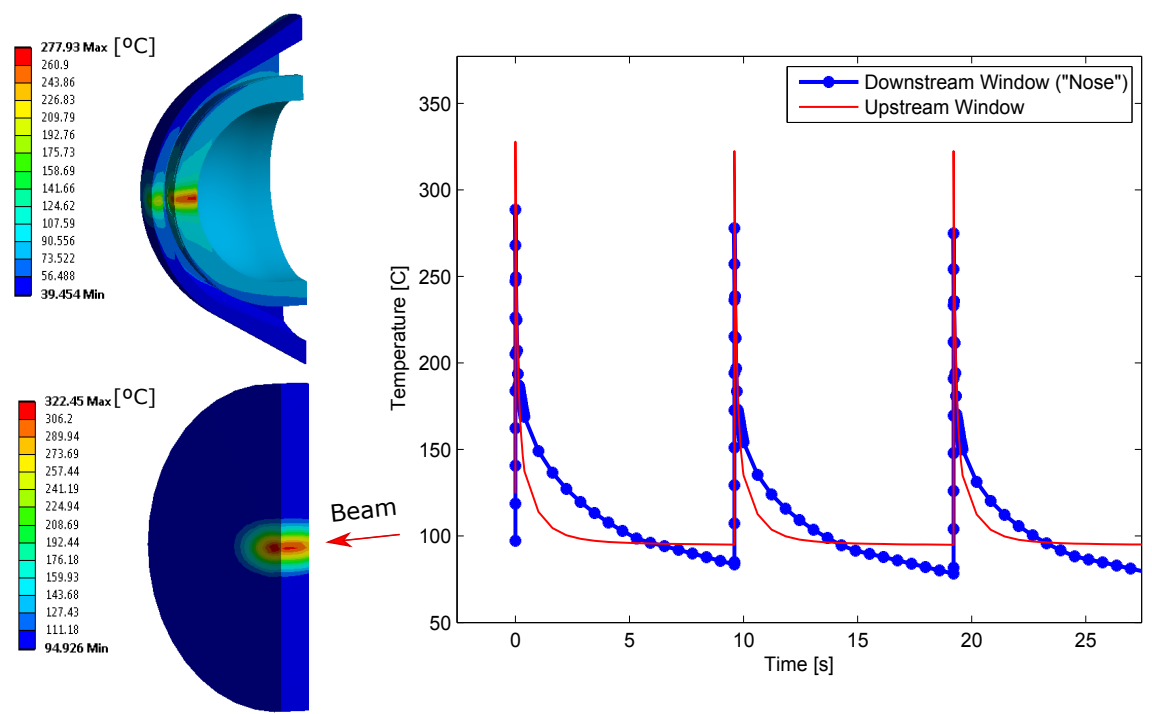

Figure 7.13: (left) Temperature profile at the end of the proton pulse impact at the upstream and downstream windows. (Right) Time history of the maximum temperature reached in the windows during three consecutive proton pulse impacts. 


\subsubsection{Stresses Reached in the Windows}

In the same way as happening in the target core, the extremely fast increase of temperature in the windows will make that the dynamic stresses dominate the quasi-static response. Transient-structural calculations of the pulse impact are therefore performed. Nevertheless, the quasi-static pre-stressed state of the windows right before the proton pulse impact due to their remaining temperature from previous pulses (figure 7.12-(c)) is also taken into account. This is done in the simulation by defining a previous load step with time integration off in which this remaining temperature profile is applied. The pressure load of 20 bars of the cooling air for the case of the double-walled downstream window is also applied during this initial load step and maintained in the transient (second time step).
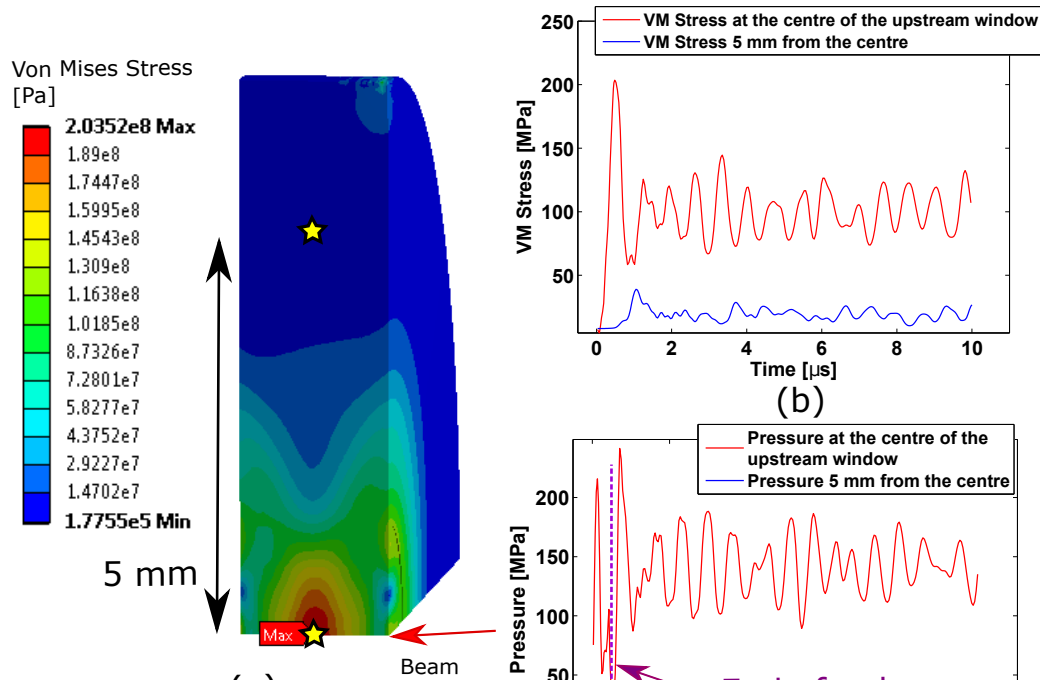

(b)

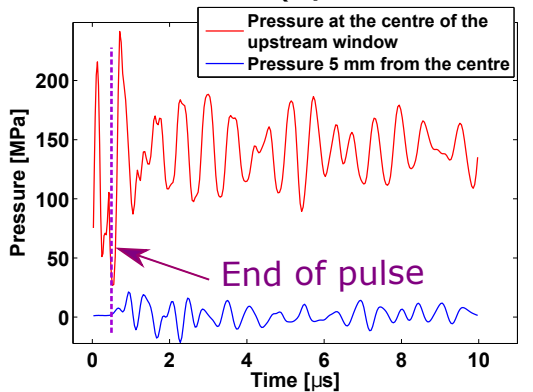

(c)

Figure 7.14: (a) Snapshot of the dynamic stress distribution in the upstream window at the end of the beam impact. (b-c) Time history of the VM stress and pressure at the center and periphery of the upstream window during $10 \mu$ s after the impact.

Figure 7.14(a-c) shows the VM stress distribution as well as the stress and pressure time transient response in the upstream Ti-6Al-4V window. The contour plot of the figure 7.14(a) correspond to the VM stress distribution right after the end of the proton pulse impact (temperature profile shown in figure 7.13). Figures 7.14(b) and (c) show the transient response of VM stress and pressure respectively during $10 \mu$ s after the pulse at the windows center (red) and $5 \mathrm{~mm}$ towards the periphery 
(blue). Maximum VM stress of $200 \mathrm{MPa}$ is reached in the center of the window at the end of the pulse burst $(0.480 \mu \mathrm{s})$. A high frequency wave with a period of $0.7 \mu \mathrm{s}$ (corresponding to the thickness of the window) dominates the dynamic response. The amplitude of this wave is significantly reduced towards the windows periphery as shown in the plot. Figure $7.14(\mathrm{c})$ shows also the pressure response at these positions. It is interesting to note the presence of end-of-pulse tensile wave as the ones described in Chapter 3. In any case, it can be seen that the wave subjects the window only to compressive states.

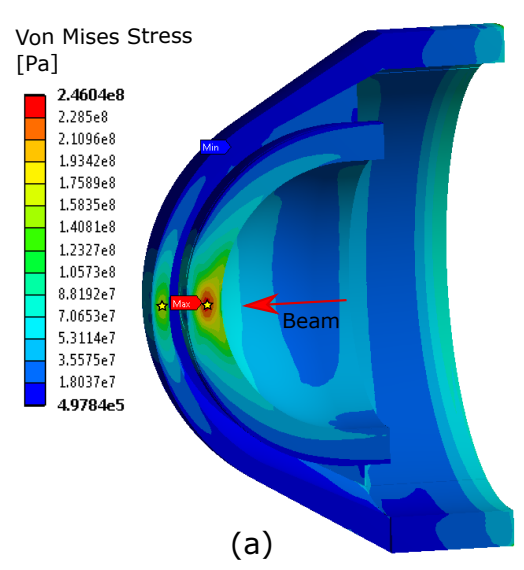

(a)

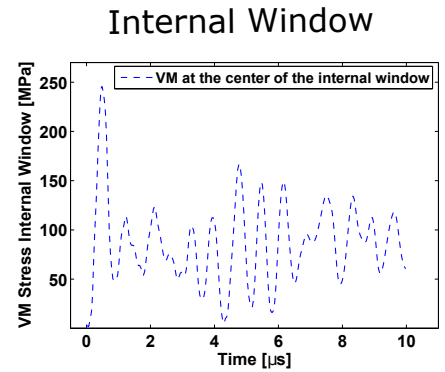

(b)

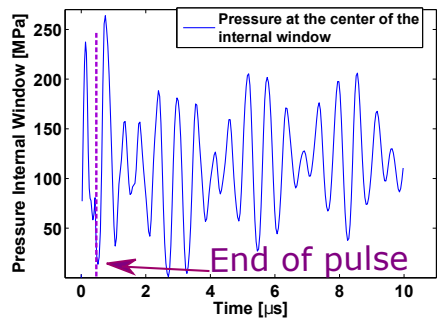

(c)

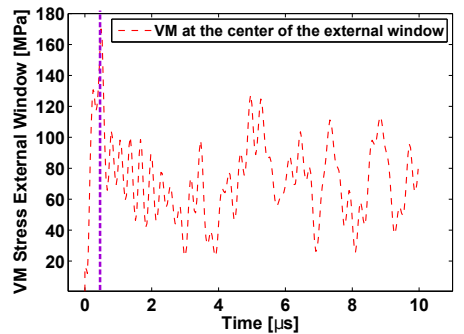

(d)

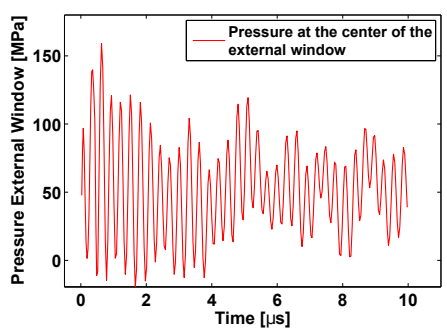

(e)

Figure 7.15: (a) Snapshot of the dynamic stress distribution in the double walled downstream window at the end of the beam impact. (b-c) Time history of the VM stress and pressure at the center of the internal downstream window during $10 \mu \mathrm{s}$ after impact. (d-e) VM stress and pressure at the center of the external downstream window. 
In a similar way, figure 7.15(a-e) shows the VM and pressure response at the downstream double-window. Figure 7.15(a) shows the VM stress distribution in the geometry at the end of the pulse. The internal window has a thickness of $2 \mathrm{~mm}$ while the external one $1 \mathrm{~mm}$. At can be seen in figures 7.15(b) and (d), the highest stresses are present in inner window, reaching $245 \mathrm{MPa}$, while 170 $\mathrm{MPa}$ are reached in the external one. Reached stresses are therefore higher in the downstream windows than in the upstream, even if temperatures in the former are slightly lower. Nevertheless, everything is also subjected only to compressive states as shown in figures 7.15(c)-(e). Also a high frequency wave dominates, with a period of $0.6 \mu \mathrm{s}$ in the inner window and only $0.3 \mu \mathrm{s}$ in the external (due to its lower thickness).

\subsubsection{Fatigue Analysis}

As shown in the previous plots, it is clear that the stresses present in the target windows are well below the yield strength of Ti-6Al-4V at $300{ }^{\circ} \mathrm{C}$, which is around $650 \mathrm{MPa}$. Fatigue damage due to continue pulse heating and induced stress waves are therefore the main concern that has to be taken into account. A detailed fatigue analysis is presented here for this purpose. For the analysis, it is considered that during 200 days of operation per year, the proton beam will be impacted every $16 \mathrm{~s}$ on average in the most conservative operation scenario. This leads to approximately $10^{6}$ proton pulses per year. In this context, looking at the results recently presented in figures 7.14 and 7.15 , it is interesting to note that each of these pulses will submit the windows to two main types of cyclic load:

i The cyclic load corresponding to the pulse burst, in which the minimum stress is the one corresponding to the steady stress state distribution right before the pulse burst, and the maximum is the one reached a few instants after the pulse impact. The maximum stresses of this cyclic load therefore correspond to $200 \mathrm{MPa}, 245 \mathrm{MPa}$ and $170 \mathrm{MPa}$ for the upstream and interior/exterior downstream windows respectively.

ii In addition to this cyclic load, it can be observed in figures 7.14 and 7.15 that each pulse leads to a dynamic response in which a very a high frequency elastic wave subjects the material to many load cycles until is damped. For the fatigue analysis, it is conservatively assumed that this high frequency elastic wave would take $30 \mathrm{~ms}$ to damp, which corresponds to $10^{5}$ oscillations/pulse $\left(10^{11} /\right.$ year $)$. These stress oscillations take place around an averaged stress level. Even if in reality the amplitude of these oscillations varies, it has been assumed that its constant and conservatively set in the maximum oscillation observed in figures 7.14-7.15. 
Table 7.2 summarizes the considered mean alternating stress for the two different types of cycles assumed at the upstream and downstream windows. Considered target lifetime is 5 years.

Table 7.2: Summary of mean and alternating stresses reached in the AD-Target windows considered in the fatigue analysis.
(i) Low freq. Cycle
(ii) High freq. Cycle

Number of Cycles

Life-time (5 years)

$$
5 \cdot 10^{6}+10^{11}
$$

\begin{tabular}{lcccc}
\hline Part & $\begin{array}{l}\text { Mean } \\
\text { Stress } \\
{[\mathrm{MPa}]}\end{array}$ & $\begin{array}{l}\text { Alternating } \\
\text { Stress [MPa] }\end{array}$ & $\begin{array}{l}\text { Mean } \\
\text { Stress } \\
{[\mathrm{MPa}]}\end{array}$ & $\begin{array}{l}\text { Alternating } \\
\text { Stress [MPa] }\end{array}$ \\
\hline $\begin{array}{l}\text { Upstream Window } \\
\begin{array}{l}\text { Downstream Window } \\
\text { Internal }\end{array}\end{array}$ & 106 & 97 & 105 & 38 \\
$\begin{array}{l}\text { Downstream Window } \\
\text { External }\end{array}$ & 123 & 77 & 78 & 48 \\
\hline
\end{tabular}

Ref. [222] is of great interest for performing the fatigue analysis given the load conditions presented. In this publication, stress-cycle (S-N) curves for electropolished Ti-6Al-4V tested at $300{ }^{\circ} \mathrm{C}$ are provided. These tests have been performed in tensile load up to $10^{8}$ cycles with a stress ratio $\mathrm{R}=0.1$ (which means that the minimum load stress is 0.1 times the highest). Tensile yield and ultimate strength at $300{ }^{\circ} \mathrm{C}$ have been evaluated in $660 \mathrm{MPa}$ and $750 \mathrm{MPa}$ respectively. S-N curve shows that failure at $10^{8}$ cycles take place under a max stress of $400 \mathrm{MPa}$, which corresponds to a mean stress of $220 \mathrm{MPa}$ and alternating stress with an amplitude of $180 \mathrm{MPa}$ when considering $\mathrm{R}=0.1$. Unfortunately, there is not available data up to $10^{11}$ cycles. Nevertheless, a extrapolation has been performed since the $\mathrm{S}$ $\mathrm{N}$ curves are almost linear when plotted in logarithmic terms [223]. Figure 7.16 shows the extrapolation performed, giving that a failure at $10^{12}$ cycles would take place under a max load of $250 \mathrm{MPa}$, corresponding to a mean load of $137 \mathrm{MPa}$ and $112 \mathrm{MPa}$ amplitude of alternating stress. This linear extrapolation is considered to be conservative since, in reality, the S-N curves are not linear but slightly convex. 


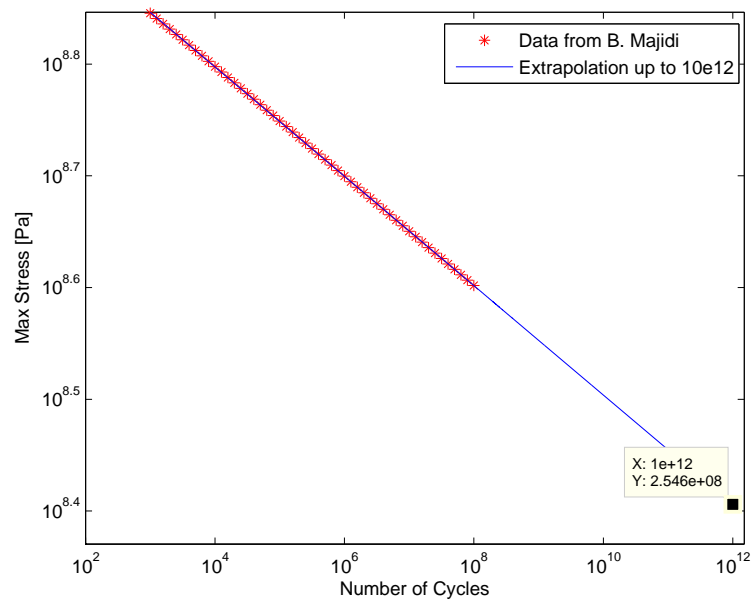

Figure 7.16: S-N curve of Ti-6Al-4V tested at $300{ }^{\circ} \mathrm{C}$ obtained from ref. [222], presented in logarithmic scale and linearly extrapolated to $10^{12}$ cycles for the present analysis. From this extrapolation, it is considered that Ti-6Al-4V would fail at $10^{12}$ cycles under a maximum load of $250 \mathrm{MPa}$ with $\mathrm{R}=0.1$.

In order to compare the data presented in table 7.2 with the one extracted from reference [222], the Goodman criterion is applied [224], which is one of the the most conservative [225]. This criterion is based in plotting the alternating load conditions in terms of the amplitude of the alternating stress $\left(\sigma_{a}\right)$ and the mean stress $\left(\sigma_{m}\right)$ in a $\sigma_{m}-\sigma_{a}$ chart. In the same chart, the so called Goodman line is also drawn following the expression 7.1:

$$
\sigma_{a}=\sigma_{e}\left[1-\left(\frac{\sigma_{m}}{\sigma_{u}}\right)\right]
$$

Where $\sigma_{e}$ is the fatigue limit corresponding to a fully reversed cycle $\mathrm{R}=-1$, i.e. when the mean stress $\sigma_{m}$ is equal to zero. $\sigma_{u}$ is the ultimate tensile strength. The Goodman line can be therefore drawn using the data from ref. [222], which provides $\sigma_{m}$ and $\sigma_{a}$ at which fatigue failure at $300{ }^{\circ} \mathrm{C}$ takes place after $10^{8}$ cycles. With this point, which corresponds to $-250,180$ - MPa, and the yield strength at $300{ }^{\circ} \mathrm{C}$ (also provided in ref. [222]) which corresponds to the point -0,750- MPa, the Goodman line is drawn as shown in figure 7.17-(a). The Goodman criterion states that no fatigue failure will take place at a given number of cycles as long as the alternating load falls below the Goodman line.

Figure 7.17(a)-(b) plots the operation points of the three target windows according to the Goodman criterion for the two types of cyclic load presented in table 7.2. 
The Goodman line of figure 7.17-(b) for $10^{12}$ cycles has been drawn using the extrapolated point obtained in figure 7.16. It can be seen that the operation points for both cycling loads fall in the safe zone. In addition, the PalmgrenMiner's rule has been applied for assessing the combination of the two cyclic loads (as the Goodman criterion only considers them independently).

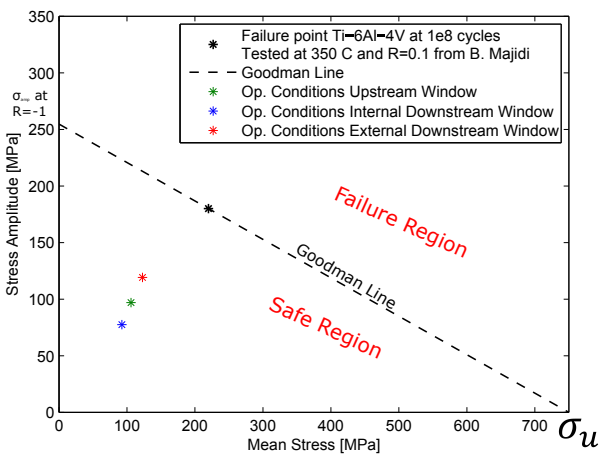

(a)

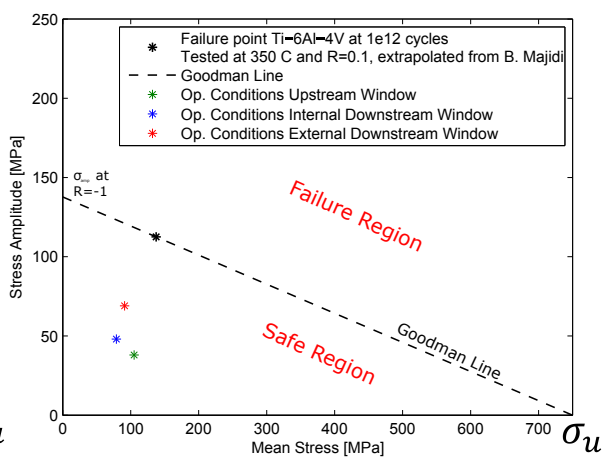

(b)

Figure 7.17: Application of Goodman fatigue criterion for the two types of cyclic loads presented in table 7.2. It can be seen that both loads fall in the safe region for the $10^{8}$ and $10^{12}$ cycles.

Palmgren-Miner's rule [226] assumes that the total life of a component can be estimated by adding up the percentage of life consumed for each given cyclic load. In order to apply this rule, it has been considered that the cyclic load (i) would fail at $10^{8}$ cycles while the load (ii) at $10^{12}$ (even if demonstrated by figures 7.17(a)-(b) that this is not the case). It can be seen that even under these very conservative and unrealistic assumptions, only $55 \%$ of the life time is exhausted when considering 5 years of operation.

The present analysis has shown that the new proposed design of the target envelope and cooling system would effectively remove the deposited power in the target core and survive under fatigue over 5 years of the most conservative operational scenario. Elecro-polishing of the envelope is foreseen in order to extend the life of the windows and to make the analysis applicable (as the fatigue data was obtained from elecro-polished samples). The assumptions considered in the fatigue analysis have been very conservative, in particular taking into account that the target windows are in reality subjected mainly to compressive loads, which are significantly more favorable than the tensile curves considered in the analysis. The only aspect that has not be taken into account is the radiation damage on the window, and its detrimental effect on the mechanical properties since, unfortunately, no fatigue data of irradiated Ti-6Al-4V tested at very high cyclic load has been found in the literature. Best study found on this corresponds to ref. [227], in which $590 \mathrm{MeV}$ 
proton irradiated Ti-6Al-4V has been fatigue tested at $350{ }^{\circ} \mathrm{C}$ up to $10^{4}$ cycles. According to this study, fatigue behavior is not significantly affected by radiation at high number of low amplitude cycles. A reduction of fatigue life $20-50 \%$ was observed only at high strains and low cycles, attributed to an increase in the stress level due to radiation induced embrittlement. Nevertheless, these high strains levels are not expected to be reached in the AD-Target windows and, even if a $50 \%$ reduction of the life time would take place, the 5 years life analysis just presented still holds.

\subsection{Conclusions of the Thesis}

Chapter 7 encloses the present thesis with different analyzes and first prototyping activities of the new AD-Target design. Acquired knowledge and lessons learned in the previous chapters have been applied for proposing a new concept of target which core is made of small rods of tantalum embedded in an EG-matrix and encapsulated in a double-envelope, air cooled, Ti-6Al-4V assembly.

This thesis has covered several multidisciplinary topics for proposing a new design of this unique device. On one hand, the thesis covers topics related to particle accelerators technology and new particle production targets, including physics behind these devices and main relevant operation parameters. Then, from the literature review presented in Chapter 1, it was clear that one of the key aspects in the antiproton production yield and main cause of its failure in the past is the survival of the high density target core material exposed to the extreme dynamic conditions every time is impacted by the primary proton beam. From this starting point, detailed studies of the response of the target core material from the mechanical point of view have been carried out. These studies tried to reduce the existing uncertainties about what happens to this high density material when exposed by the proton beam impact to a rise of temperature of $2000{ }^{\circ} \mathrm{C}$ in less than $0.5 \mu \mathrm{s}$ and the successive generated pressure waves. To answer these questions, very specific computational tools named hydrocodes (which are applied normally to highly dynamic phenomena such as simulations of ballistics or explosions) have been used.

The hydrocode analysis of the target core response presented in Chapter 3 have brought important insights on the material response at a very high level of detail. The material characterization of pure iridium (which was not found in the literature) and implementation of strength models in the hydrocodes, which take into account the material response beyond plastic deformation at high strain rate and temperatures, have allowed obtaining accurate simulations and learning that a high frequency radial compressive-to-tensile pressure wave is generated after each pulse. In addition, it has been discovered the presence of end-of-pulse tensile waves and their fundamental role for being responsible of the observed compressive- 
to-tensile pressure waves. Knowledge about this phenomenon could be used for finding strategies to reduce the magnitude of the reached tensile stresses, such as the use of claddings or increasing the core diameter. These findings can be also valuable for the design of other types of BIDs consisting in thin rods exposed to a highly dynamic load. In addition, these hydrocodes simulations have shown that not only the target core is subjected to stresses which compromise its structural integrity, but also the containing graphite matrix close to the core interface, due to the transmission of the radial pressure wave from the core.

It has been also shown that, even if the hydrocode simulations are a very powerful tool, numerical simulations are not enough to answer some of the key questions regarding the material response of the $\mathrm{AD}$-Target core, such as which is its mode of fracture at the exposed conditions and at which level of stresses the fracture takes place, as well as the influence of this fracture in the effective density and antiproton production. In addition, unavoidable extrapolation of the strength models in the simulations brought concerns about their accuracy. For this reason, a challenging first-of-its-kind experiment called HRMT27 and described in Chapters 4, 5 and 6 was carried out. In this experiment, several rods of high density materials such as Ir, W, W-La, Mo, TZM and Ta where brought to equivalent conditions as reached in the AD-Target core by impacting them with $440 \mathrm{GeV}$ protons beams using the HiRadMat facility at CERN. Extensive online instrumentation measured displacement, velocity and temperature at their surfaces with an acquisition rate of $\mathrm{MHz}$ and despite the high levels of prompt radiation involved. The design and implementation of the experiment described in Chapter 4 was a breakthrough by itself and has brought useful experience, such as the use of passive devices like optical heads and fibers for data acquisition resistant to radiation, finding polishing technologies to obtain roughness in the order of nanometers in very hard materials like iridium, or the use of accurate beam position monitors BTVBPKG for this type of experiments. In addition, high quality data of the predicted radial wave was measured and confirmed the accuracy of the hydrocode simulations performed. All of the irradiated target materials except tantalum showed internal cracking from conditions 5-7 times below the present in the AD-Target. Not only that, the observed changes in the measured radial waves when progressively increasing the impacted intensity allowed to find thresholds of damage in the materials of interest. In this way, failure models for tungsten and iridium were benchmarked. Massive fragmentation observed in the iridium target exposed to equivalent $\mathrm{AD}$-Target strongly suggests that core of the aforementioned target is cracked from the first received pulse and that the effective antiproton production can be substantially reduced by this fact in the current operation. Tantalum, on the other hand, apparently survived the AD-Target conditions without presenting internal cracking thanks to its high ductility, and for this reason it became the baseline core material for the new design. 
A conceptual design and first prototyping activities of the new AD-Target were presented in the last chapter. In this design, the core of the target is made of different small rods of tantalum with different diameters. Larger diameters than the current $3 \mathrm{~mm}$ rod are proposed to try to reduce the tensile pressures in the core. In addition, the target is embedded in a matrix of compressed Expanded Graphite (EG) instead of graphite. The high flexibility of EG is expected to be beneficial for having deformation instead of fracture in the containing matrix, and also for damping the radial wave. Furthermore, its pre-compressed state could provide a very good contact with the core interface due to Poisson effect and guarantee a good thermal contact between the EG matrix and the tantalum core even if plastic deformation takes place in the latter. A first scaled prototype following this concept has been successfully built and it will be tested in HiRadMat soon to verify these hypothesis. Another important difference in the new proposed design in comparison with the current one is the cooling system, which has been replaced by a serpentine circulated by air at 20 bars. CFD and thermo-mechanical calculations have been carried out to validate this concept and to define the cooling flow characteristics. A detailed fatigue analysis of the target windows, guaranteeing 5 years of operation is also included.

There are still several open points left in this thesis. First, the induced radiation damage and its influence in the mechanical properties of the target core has not been studied in detail. This is due to the lack of literature regarding high energy proton induced radiation damage, even less in the materials involved such as iridium. In addition, it was considered that, due to the high stresses reached in the core even from the first pulse, relevance of radiation induced damage will take place at long term and with a secondary influence. It is true, however, that the better expected performance of tantalum could then make radiation induced damage more relevant. Nevertheless, aperture of one of the spent Ir targets which has been in operation for year is foreseen. This analysis of the irradiated target core could bring interesting insights on changes in the material micro-structure and properties due to radiation damage which could help for understanding better this concern. In addition, a $280 \mathrm{MeV}$ proton irradiation campaign to study radiation damage in high density materials which are candidate for the AD-Target core has been launched. This irradiation is currently taking place using the BLIP facility at Brookhaven National Laboratory (BNL) in US in the context of the RaDIATE international collaboration [228].

Another open point left in the thesis is the long term survival of Ta as target material when impacted by a large number of pulses, since the material will undergo plastic deformation in each pulse and successive hardening could brittle the material. Unfortunately, the HRMT27 experiment did not have the chance to subject the Ta target to a high number of proton pulse impacts enough to observe this effect. In that sense, the new prototype of Ta-embedded in EG described in last chapter will be irradiated again in HiRadMat soon and impacted by a higher num- 
ber of pulses to try to study this effect during the subsequent PIEs. Furthermore, another HiRadMat experiment testing several prototypes of AD-Target is foreseen in 2018. This experiment, called PROTAD, will test different core configurations, in terms of small rods of different diameters embedded in a EG matrix. Finally, the construction of more than one definitive AD-Target, with different core configurations (even still using iridium instead of tantalum), is also foreseen. In this way, tests during the first months of real operation could be done to verify which solution gives the highest antiproton production. 


\section{Bibliography}

[1] T. Eriksson. AD Consolidation for operation beyond 2010. Tech. rep. CERNAB-2008-068-OP. Geneva: CERN, 2008 (cit. on p. 1).

[2] P. Larochelle. "Machines and Methods for Trapping Antihydrogen". PhD thesis. MIT, 2010 (cit. on p. 2).

[3] http://asacusa.web.cern.ch/ASACUSA/ (cit. on p. 2).

[4] M. Amoretti et al. "Production and detection of cold antihydrogen atoms". In: Nature 419.6906 (2002), pp. 456-459. ISSN: 0028-0836. DOI: 10.1038/ nature01096 (cit. on p. 2).

[5] http://alpha.web.cern.ch/ (cit. on p. 2).

[6] S. Aghion et al. "Detection of low energy antiproton annihilations in a segmented silicon detector". In: Journal of Instrumentation 9.06 (2014), P06020 (cit. on p. 2).

[7] V. Chohan et al. Extra Low ENergy Antiproton (ELENA) ring and its Transfer Lines: Design Report. Geneva: CERN, 2014 (cit. on p. 2).

[8] T Eriksson et al. "Upgrades and consolidation of the CERN AD for operation during the next decades". In: CERN-ACC-2013-0036 (2013), 3 p (cit. on p. 3).

[9] C. Torregrosa, A. Perillo-Marcone, and M. Calviani. Designs Characteristics and Main Inherent Concerns of the Antiproton Decelerator Target. Tech. rep. CERN, 2015 (cit. on p. 3).

[10] M. Calviani and E. Nowak. FLUKA implementation and preliminary studies of the AD-target area. Tech. rep. 2012 (cit. on pp. 3, 5, 9, 17, 66). 
[11] D. Möhl. "Production of low-Energy Antiprotons". In: Hyperfine Interaction 109 (1997), pp. 33-41 (cit. on pp. 3, 6, 10, 15).

[12] M.R. Bellone et al. The Design and Prototype Test of the CERN Antiproton Production Target. Tech. rep. SPS/80-9/ABT. Geneva: CERN, 1980 (cit. on pp. $3,7,8,14)$.

[13] I. Efthymiopoulos et al. "HiRadMat: A new irradiation facility for material testing at CERN". In: San Sebasti $\tilde{A}_{j} n$, Spain: Proceedings of IPAC2011, 2011, pp. 1665-1667 (cit. on pp. 4, 99).

[14] H. Koziol and D. Möhl. "The CERN Low-Energy Antiproton Programme: the Synchrotrons". In: Phys. Rep. 403-404.CERN-AB-2004-098-ADM (2004), 271-280. 11 p (cit. on p. 5).

[15] S. Gilardoni. Fifty years of the CERN Proton Synchrotron: Volume 2. Comments: 58 pages, published as CERN Yellow Report. Geneva: CERN, 2013 (cit. on p. 5).

[16] K. Knie et al. "Concept for the Antiproton Production Target at FAIR". In: Conf. Proc. C1205201 (2012), WEPPD030. 3 p (cit. on p. 5).

[17] P. Sievers. Some remarks concerning the $\bar{p}-$ production target. Tech. rep. SPS-ABT-Tech-Note-78-11. Geneva: CERN, 1978 (cit. on pp. 6, 13).

[18] M. Hori and J. Walz. "Physics at CERN's Antiproton Decelerator". In: Prog. Part. Nucl. Phys. 72 (2013), pp. 206-253 (cit. on p. 6).

[19] T.W. Eaton. A Comparison between the Observed Decreases in Antiproton Yield from Passive Targets and the Theoretical Predictions Based upon the Concept of Stress and Temperature Enhanced Void Growth. Tech. rep. PS/AA/Note 85-9. Geneva: CERN, 1985 (cit. on pp. 6, 12, 13, 15).

[20] T.W. Eaton. Radiation Damage in Antiproton Targets. Tech. rep. PS/AA/Note 85-4. Geneva: CERN, 1985 (cit. on pp. 6, 15).

[21] C.D. Johnson, E. Jones, and T.R. Sherwood. "Antiproton yield expectations for the ACOL project". In: CERN-PS-87-19-AA (1987), 3 p (cit. on p. 6).

[22] P. Sievers. Elastic stress waves in matter due to rapid heating by an intense high-energy particle beam. Tech. rep. CERN-LabII-BT-74-2. Geneva: CERN, 1974 (cit. on pp. 6, 41). 
[23] C.D. Johnson. "Present and Future Possibilities of Antiproton Production from Fixed Targets at CERN". In: Hyperfine Interaction 44 (1998), pp. 2130 (cit. on pp. $7-10,13,29$ ).

[24] E Jones. "ACOL, CERN's upgrade of the antiproton accelerator complex". In: CERN-PS-86-30-AA (1986), 15 p (cit. on pp. 7-9).

[25] C. Johnson. A self-focusing mercury jet target. Tech. rep. CERN-NUFACTNOTE-113. Geneva: CERN, 2002 (cit. on p. 8).

[26] T.W. Eaton et al. "Conducting targets for $\bar{p}$ production of ACOL: past experience and prospects". In: CERN-PS-85-18-AA (1985), 3 p (cit. on p. 8).

[27] T.W. Eaton, C.D. Johnson, and R. Jones. Recent work on the production of antiprotons from pulsed current targets. Tech. rep. CERN-PS-86-15-AA. Geneva: CERN, 1986 (cit. on pp. 8, 9).

[28] D Boimond et al. Consolidation of the $400 \mathrm{kA}$ magnetic horn for AAC antiproton production. Tech. rep. CERN-PS-94-02-AR. Geneva: CERN, 1994 (cit. on pp. 9, 10).

[29] A.H. Sullivan. The Release of Radioactivity from Rhenium Targets in AA. Tech. rep. TIS-RP-TM-85-40. CERN, 1985 (cit. on pp. 9, 16, 226).

[30] C.D. Johnson. "Experience with High Flux Targets - Summary of presentation". In: Princeton, 1998 (cit. on pp. 9, 12).

[31] G. Carron et al. "Recent machine studies and improvements of the CERN antiproton accumulator". In: CERN-PS-87-23-AA (1987), 3 p (cit. on p. 10).

[32] M. Calviani. AD Target Area Summary Status, a First Analysis from EN/STI. CERN internal presentation. 2011 (cit. on pp. 10, 11).

[33] T. Jenkins and T.W. Eaton. Adiabatic Beam Heating in the Pre-stressed Antiproton Production Target. Tech. rep. CERN, 1985 (cit. on p. 13).

[34] T.W. Eaton and C. Carter. The Practical Difficulties Inherent in the Design of a Reliable Active Solid Antiproton Target for ACOL. Tech. rep. PS/AA/Note 85-11. Geneva: CERN, 1985 (cit. on pp. 13, 15). 
[35] T.W. Eaton, F. Gamba, and M. Ross. A closed circuit air cooling system for the new design of passive targets for ACOL. Tech. rep. PS/AA/Note 86-5. CERN, 1986 (cit. on p. 13).

[36] L. Davison. Fundamentals of Shock Wave Propagation in Solids. Shock Wave and High Pressure Phenomena. Springer Berlin Heidelberg, 2008. ISBN: 9783540745693 (cit. on p. 13).

[37] B. Autin et al. Design study of a proton-antiproton colliding beam facility. Tech. rep. CERN-PS-AA-78-3. 1978 (cit. on p. 14).

[38] T.W. Eaton. Some metallurgical aspects of antiproton targets. Tech. rep. PS-AA-Note-86-12. Geneva: CERN, 1986 (cit. on pp. 14, 23).

[39] S.S. Hecker, D.L. Rohr, and D.F. Stein. "Brittle fracture in iridium". In: Metallurgical Transactions A 9.4 (1978), pp. 481-488 (cit. on pp. 14, 21).

[40] Y.W. Chang, J. Guildys, and S.H. Fistedis. Two-dimensional Hydrodyamic Analysis for Primary Containment. Tech. rep. Argone National Laboratory, 1969 (cit. on p. 15).

[41] T.W. Eaton. The Significance of the Specific Acoustic Impedance of Materials in determining the magnitude of the Major Shock Wave Effects in Antiproton Targets. Tech. rep. PS/AA/Note 85-1. Geneva: CERN, 1985 (cit. on p. 15).

[42] T.W. Eaton. An Estimation of the Loss in Antiproton Yield Caused by Decreases in Target Density over Times Equal to the Duration of the Proton Pulse. Tech. rep. PS/AA/Note 86-3. Geneva: CERN, 1986 (cit. on pp. 15, 29).

[43] G.S. Was. Fundamentals of Radiation Materials Science: Metals and Alloys. Springer Berlin Heidelberg, 2007. ISBN: 9783540494720 (cit. on p. 16).

[44] T.W. Eaton et al. Calculations of beam energy deposition, adiabatic temperature rise and thermal stress in the windows of high density passive targets. Tech. rep. PS-AA-Note-85-14. Geneva: CERN, 1985 (cit. on p. 17).

[45] A. Ferrari et al. FLUKA: A multi-particle transport code (program version 2011). Geneva: CERN, 2011 (cit. on pp. 17, 44). 
[46] A. Bertarelli. An analytical model to study transient thermal stresses in graphite target rods hit by off-axis beam for cngs facility. Tech. rep. ESTME-2003-006. CERN, 2003 (cit. on pp. 18, 41).

[47] J. Morgan. Pbar Production Target Performance in Run II. Tech. rep. Illinois, US: Pbar Note 683, Fermilab, 2003 (cit. on pp. 18, 19).

[48] B.W. Riemer. The Spallation Neutron Source, Target Design and Operating Experience. Presented at A\&T Seminar CERN. Sept. 2013 (cit. on p. 18).

[49] Particle Accelerators for Science and Innovation UK wiki (cit. on p. 18).

[50] M. Calviani et al. Conceptual Design of the SHiP Target and the Target Complex. Tech. Proposal Annex SHIP-TP-2015-A4 EDMS. 1465053. CERN, 2015 (cit. on p. 18).

[51] M.D. Church and J.P. Marriner. "THE ANTIPROTON SOURCES: Design and Operation". In: Annu. Rev. Nucl. Part. Sci. 43 (1993), p. 253 (cit. on pp. 18, 20).

[52] B.C. Brown et al. "Fermilab main injector: High intensity operation and beam loss control". In: Phys. Rev. ST Accel. Beams 16 (2013), p. 071001 (cit. on p. 18).

[53] J. Morgan. "FNAL Pbar Target Station. Overview and Target Issues". In: FNAL Pbar Target Station. High-Power Targetry Workshop, 2003 (cit. on p. 19).

[54] E.K. Ohriner. "Processing of Iridium and Iridium Alloys". In: Platinum Metals Rev. 52.3 (2008), pp. 186-197 (cit. on p. 20).

[55] N.K. Wahl. "Natural-convective high-temperature oxidation of iridium". MA thesis. Montana State University, 1974 (cit. on p. 20).

[56] L.B. Hunt. In: Platinum Metals Rev. 31 (1987), pp. 32-41 (cit. on p. 20).

[57] B. Fischer, A. Behrends, and D. Freund. "High Temperature Mechanical Properties of the Platinum Group Metals". In: Platinum Metals Rev. 43 (1999), pp. 18-28 (cit. on p. 21).

[58] H. Inouye. "Platinum Group Alloy Containers for Radioisotopic Heat Sources". In: Platinum Metals Rev. 23 (1979), pp. 100-108 (cit. on p. 21). 
[59] B.L. Mordike and C.A. Brookes. "The tensile properties of iridium at high temperatures". In: Platinum Metals Rev. 4 (1960), pp. 94-99 (cit. on p. 21).

[60] P. Panfilov and A. Yermakov. "Mechanisms of inherent and impurity induced brittle intercrystalline fracture in pure FCC-metal iridium". In: International Journal of Fracture 128.1 (2004), pp. 147-151 (cit. on p. 21).

[61] P. Panfilov and A. Yermakov. "On brittle fracture in polycrystalline iridium". In: Journal of Materials Science 39.14 (2004), pp. 4543-4552 (cit. on p. 21).

[62] T.C. George and M.F. Stevens. High temperature impact properties of DOP26 iridium. Tech. rep. 77980253. Los Alamos National Laboratory, 1988 (cit. on p. 21).

[63] T.C. George. High-strain-rate, High-temperature Biaxial Testing of DOP26 Iridium. Tech. rep. LA-11065. Los Alamos National Laboratory, 1988 (cit. on p. 21).

[64] B. Song et al. Dynamic High-Temperature Characterization of an Iridium Alloy in Tension. Tech. rep. Report: SAND2015-8380. Sandia National Laboratory, 2015 (cit. on pp. 21, 69).

[65] B. Song et al. "Dynamic High-temperature Testing of an Iridium Alloy in Compression at High-strain Rates". In: Strain 50 (2014), pp. 539-546 (cit. on pp. 21, 69).

[66] J.H. Schneibel, C. . Carmichael, and E.P. George. High Strain Rate Tensile Testing of DOP-26 Iridium. Tech. rep. Report ORNL/TM 2007/81. Oak Ridge National Laboratory, 2007 (cit. on pp. 21, 69).

[67] M. Scapin et al. "Experimental results and strength model identification of pure iridium". In: International Journal of Impact Engineering 106 (2017), pp. 191-201 (cit. on pp. 21, 70, 72, 73, 90, 192).

[68] H.R. Ogden F.F. Schmidt. The engineering properties of tungsten and tugnsten alloys. Tech. rep. DMIC Report 191. Batelle Memorial Institute, Defence Metals Information Center, 1963 (cit. on pp. 21, 22).

[69] E. Lassner and W.D. Schubert. Tungsten: Properties, Chemistry, Technology of the Element, Alloys, and Chemical Compounds. Springer US, 2012. ISBN: 9781461549079 (cit. on p. 22). 
[70] A.M. Lennon and K.T. Ramesh. "The thermoviscoplastic response of polycrystalline tungsten in compression". In: Material Science and Engineering A276 (1998), pp. 9-21 (cit. on pp. 22, 69, 70).

[71] T. Dummer et al. "Effect of strain rate on plastic flow and failure in polycrystalline tungsten". In: Acta Materialia 46.17 (1998), pp. 6267 -6290. ISSN: 1359-6454 (cit. on p. 22).

[72] Y. Sahin. "Recent Progress in Processing of Tungsten Heavy Alloys". In: Journal of Powder Technology 2014 (2014) (cit. on p. 22).

[73] K. T. Ramesh and R.S. Coates. "Microstructural influences on the dynamic response of WHAs". In: Metal. Trans. 23A (1992), p. 2625 (cit. on p. 22).

[74] A. Zurek and G. Gray III. "Dynamic strength and strain rate effects on fracture behavior of tungsten and tungsten alloys". In: Journal de Physique IV Colloque 01.C3 (1991), pp. 631-637 (cit. on p. 22).

[75] M. Scapin. "Mechanical characterization and modeling of the heavy tungsten alloy IT180". In: International Journal of Refractory Metals and Hard Materials 50 (2015), p. 258 (cit. on p. 22).

[76] J. Das, G. Appa Rao, and S.K. Pabi. "Microstructure and mechanical properties of tungsten heavy alloys". In: Materials Science and Engineering: A 527.29-30 (2010), p. 7841 (cit. on p. 22).

[77] S. Yadav and K.T. Ramesh. "The mechanical properties of tungsten-based composites at very high strain rates". In: Materials Science and Engineering: A 203 (1995), pp. $140-153$ (cit. on p. 22).

[78] S.R. Chen and G.T. Gray. "Constitutive behavior of tungsten and tantalum: experiment and modelling". In: Proceedings of 2nd International Conference on Tungsten and Refractory metals. p. 489-498. Princeton: Metals Powder Industries Federation, 1995 (cit. on pp. 22, 25, 70, 71, 189, 190, 194).

[79] G.P. Skoro et al. "Yield strength of molybdenum, tantalum and tungsten at high strain rates and very high temperatures". In: Journal of Nuclear Materials 426.1-3 (2012), pp. 45-51 (cit. on pp. 22, 98).

[80] D.H. Lassila, F. Magness, and D. Freeman. Ductile-Brittle Transition Temperature testing of tungsten using the three-point bend test. Tech. rep. UCRLID-108258. Lawrence Livermore National Lab., 1991 (cit. on p. 22). 
[81] D.H. Lassila and G.T. Gray III. "Ductile-brittle transition behavior of tungsten under shock loading". In: J. Physique IV 4 (1994), pp. C8-349 (cit. on p. 22).

[82] J.M. Steichen. "Tensile properties of neutron irradiated TZM and tungsten". In: Journal of Nuclear Materials 60.1 (1976), pp. 13 -19 (cit. on pp. 22, 28).

[83] S.A. Maloy et al. "The Effect of $800 \mathrm{MeV}$ Proton Irradiation on the Mechanical Properties of Tungsten". In: Material Transactions 43.4 (2002), pp. 633-637 (cit. on p. 22).

[84] M. Rieth et al. "Review on the EFDA programme on tungsten materials technology and science". In: Journal of Nuclear Materials 417.1-3 (2011). Proceedings of ICFRM-14, pp. 463-467 (cit. on p. 23).

[85] "Recent progress in research on tungsten materials for nuclear fusion applications in Europe". In: Journal of Nuclear Materials 432.1-3 (2013), pp. 482-500 (cit. on p. 23).

[86] S. Buono, C. Catinaccio, and C. Rubbia. A tungsten-Rhenium alloy as a beam window material for the energy amplifier. Tech. rep. CERN-ETINTERNAL-NOTE-96-024. Geneva: CERN, 1996 (cit. on p. 23).

[87] Y. Nemoto et al. "Microstructural development of neutron irradiated W-Re alloys". In: J. Nucl. Mater. 283-287 (2000), pp. 1144-1147 (cit. on p. 23).

[88] M. Fokuda et al. "Microstructural development of tungsten and tungstenrhenium alloys due to neutron irradiation in HFIR". In: J. Nucl. Mater. 455 (2014), pp. 460-463 (cit. on p. 23).

[89] L.L. Snead. In: The Effect of Neutron Irradiation on PFCs at DEMORelevant Conditions. San Diego US: 19th Plasma Surface Interactions Conference, Journal of Nuclear Materials, 2010 (cit. on p. 23).

[90] J.W.G. Thomason. "Upgrades to ISIS for the new second target station". In: Proceedings of EPAC08 (2008) (cit. on pp. 23, 131).

[91] M. Calviani. Preliminary Conceptual Design of the nTOF Spallation Target \#3. Tech. rep. TOF-TAR-EN-0001. CERN, 2016 (cit. on pp. 23, 131). 
[92] J.R.J. Bennett. "UK Studies of Solid Targets for Neutrino Factories". In: Nuclear Physics B - Proceedings Supplements 149 (2005), pp. 262 -264 (cit. on pp. 23, 131).

[93] S. Peggs and R. Kreier. ESS Technical Design Report. ESS, 2012 (cit. on pp. 23, 131).

[94] G. De Lellis. "SHiP: a new facility with a dedicated detector for neutrino physics". In: PoS EPS-HEP2015.CERN-SHiP-PROC-2016-009 (2015), p. 037 (cit. on pp. 23, 27, 28, 131, 133).

[95] M. Mabuchi et al. "Tensile properties at elevated temperature of W-La2O3". In: Materials Science and Engineering: A 214.1 (1996), pp. $174-176$ (cit. on p. 24).

[96] M. Fukuda et al. "Microstructure development of dispersion-strengthened tungsten due to neutron irradiation". In: Journal of Nuclear Materials 449.1-3 (2014), pp. $213-218$ (cit. on p. 24).

[97] V. Shirokova et al. "Comparison of damages in tungsten and tungsten doped with lanthanum-oxide exposed to dense deuterium plasma shots". In: Journal of Nuclear Materials 435.1-3 (2013), pp. 181 -188 (cit. on p. 24).

[98] M. Rieth and B. Dafferner. "Limitations of $\mathrm{W}$ and W-1\%La2O3 for use as structural materials". In: Journal of Nuclear Materials 342.1-3 (2005), pp. $20-25$ (cit. on p. 24).

[99] S.M. Cardonne et al. "Tantalum and its alloys". In: International Journal of Refractory Metals and Hard Materials 13.4 (1995), pp. 187 -194. ISSN: 0263-4368 (cit. on p. 24).

[100] Defense Metal Information Center. The Engineering Porperties of Tantalum and Tantalum Alloys. Tech. rep. DMIC Report 189. Battelle Memorial Institute, 1963 (cit. on p. 24).

[101] Mechanical Properties of Pure Tungsten. PLANSEE webpage. 0 (cit. on pp. 24, 79).

[102] S. Nemat-Nasser, J.B. Isaacs, and M. Liu. "Microstructure of high-strain, high-strain-rate deformed tantalum". In: Acta Mettalurgica Inc. 46.4 (1998), pp. 1307-1325 (cit. on p. 25). 
[103] K.G. Hoge and A.K. Mukherjee. "The temperature and strain rate dependence of the flow stress of tantalum". In: Journal of Materials Science 12.8 (1977), pp. 1666-1672 (cit. on p. 25).

[104] G. Regazzoni and F. Montheillet. "Mechanical properties at high rates of strain". In: Proceedings of the 3rd conference on the mechanical properties of materials at high rates in Oxford. 1984 (cit. on p. 25).

[105] F.J. Zerilli and R.W. Armstrong. "Description of tantalum deformation behavior by dislocation mechanics based constitutive relations". In: Journal of Applied Physics 68.4 (1990), pp. 1580-1591 (cit. on p. 25).

[106] G.T. Gray III et al. Constitutive Equations for Annealed Metals Under Compression at High Strain Rates and High Temperatures. Tech. rep. LA12669-MS. Los Alamos National Laboratory, 1994 (cit. on p. 25).

[107] K.S. Vecchio. "High-strain, high-strain-rate deformation of tantalum and tantalum-tungsten alloys". In: Journal de Physique IV C8 (1994), p. 301 (cit. on p. 25).

[108] L.E. Murr et al. "Shock-induced deformation twinning in tantalum". In: Acta Metallurgica Inc. 45.1 (1997), pp. 157-175 (cit. on p. 25).

[109] R. Kapoor S. Nemat-Nasser. "Deformation behavior of tantalum and a tantalum tungsten alloy". In: International Journal of Plasticity 17 (2001), pp. 1351-1366 (cit. on p. 25).

[110] G. Ravichandran. "Influence of Processing on the High Strain Rate Behavior of Refractory Metals - A Review". In: Materials and Manufacturing Processes 9.6 (1994), pp. 1031-1046 (cit. on p. 25).

[111] L. Hojun et al. "Physically-based strength model of tantalum incorporating effects of temperature, strain rate and pressure". In: Modelling and Simulation in Materials Science and Engineering 24.5 (2016), p. 055018 (cit. on p. 25).

[112] N.R. Barton et al. "A multiscale strength model for extreme loading conditions". In: Journal of Applied Physics 109 (2011), p. 073501 (cit. on p. 25).

[113] R. Becker et al. A tantalum strength model using a multiscale approach: version 2. Tech. rep. LLNL-TR-417075. Lawerence Livermore National Laboratory, 2009 (cit. on p. 25). 
[114] D. Errandonea. "Can metals be a liquid glass?" In: Nature Materials 8 (2009), pp. $170-171$ (cit. on p. 25).

[115] M.D. Furnish et al. "Variability in Dynamic Properties of Tantalum: Spall, Hugoniot Elastic Limit and Attenuation". In: AIP Conf. Proc. 845 (2006), pp. 615-618 (cit. on p. 25).

[116] F. Llorca, G. Roy, and P. Antoine. "Ductile damage of tantalum under spalling effects. Experimental and metallurgical analysis". In: J. Physique IV 10 (2000), p. 775 (cit. on p. 25).

[117] A.K. Zurek et al. "Micromechanics of spall and damage in tantalum". In: Journal of Materials Processing Technology 60.1 (1996), pp. 261 -267 (cit. on p. 25).

[118] W.R. Thissell et al. "Dynamic Failure Resistance of Two Tantalum Materials with Different Melt Practice Sequences". In: Shock Compression of Condensed Matter 706 (2003), p. 495 (cit. on p. 25).

[119] S.V. Razorenov et al. "The spall strength and Hugoniot elastic limit of tantalum with various grain size". In: Shock Compression of Condensed Matter 1426 (2012), pp. 991-994 (cit. on p. 25).

[120] F.W. Wiffen. "The microstructe and swelling of neutron irradiated tantalum". In: Journal of Nuclear Materials 67 (1977), pp. 119-130 (cit. on p. 26).

[121] M.L. Grossbeck and F.W. Wiffen. Swelling and tensile properties of EBRII-irradiated tantalum alloys for space reactor applications. Tech. rep. CONF850103-10. Oak Ridge National Laboratory, 1987 (cit. on p. 26).

[122] R.D. Brown J.R. Cost. "Mechanical properties of $800 \mathrm{MeV}$ proton-irradiated metals". In: Effect of Radiation on Material Properties: 11th International Symposium. Ed. by ASTM STP. Vol. 782. Philadephia, 1982, pp. 917-926 (cit. on p. 26).

[123] R.D. Brown, M.S. Wechsler, and C. Tschalar. "Tensile properties of several $800 \mathrm{MeV}$ proton-irradiated bcc metals and alloys". In: Effect of Radiation on Material Properties: 13th International Symposium (Part II). Ed. by ASTM STP. Vol. 956. Philadephia, 1987, pp. 131-140 (cit. on p. 26). 
[124] H. Ullmaier and F. Carsughi. "Radiation damage problems in high power spallation neutron sources". In: Nuclear Instruments and Methods in Physics Research B 101 (1995), pp. 406-421 (cit. on p. 26).

[125] J. Chen et al. "Mechanical properties of pure tantalum after $800 \mathrm{MeV}$ proton irradiation". In: Journal of Nuclear Materials 298 (2001), pp. 248-254 (cit. on p. 26).

[126] N. Simos et al. "Studies of material properties under irradiation at BNL linear isotope producer (BLIP)". In: Proceedings of HB2010, Morschach, Switzerland (2010) (cit. on p. 26).

[127] N. Simos. BLAIRR. Presentation at BLAIRR Forum. Brookhaven National Laboratory. 2016 (cit. on p. 26).

[128] J.A. Shields. Applications of Molybdenum Metal and its Alloys. IMOA International Molybdenum Association. 2013 (cit. on p. 27).

[129] E.L. Baker et al. "Development of Molybdenum Shaped Charge Liners". In: Proceedings of the TMS Symposium on Molybdenum and Molybdenum Alloys. 1988 (cit. on p. 27).

[130] Mechanical Properties of Molybdenum and its Alloys. PLANSEE webpage. 0 (cit. on pp. 27, 28).

[131] J.D. Campbell and T.L. Briggs. Dep. of Engineering Sci. Tech. rep. 1969 (cit. on p. 27).

[132] S.R. Chen, P. J. Maudlin, and G.T. Gray III. Mechanical properties and constitutive relations for molybdenum under high-strain-rate deformation. Tech. rep. LA-UR-98-1870. Los Alamos National Laboratory, 1998 (cit. on p. 28).

[133] C. Chen et al. "A comparative study of a back propagation artificial neural network and a Zerilli-Armstrong model for pure molybdenum during hot deformation". In: International Journal of Refractory Metals and Hard Materials 25.5-6 (2007), pp. 411 -416. ISSN: 0263-4368 (cit. on p. 28).

[134] G.I. Kanel et al. "Spall strength of molybdenum single crystals". In: Journal of Applied Physics 74 (1993), p. 7162 (cit. on p. 28). 
[135] K. Baumung et al. "Beam-Matter Experiments with High-Power Proton Beams on KALIF". In: Shock Waves Marseille III: Shock Waves in Condensed Matter and Heterogeneous Media (1995), pp. 261-266 (cit. on p. 28).

[136] L.B. Lundberg. A Critical Evaluation of Molybdenum and Its Alloys for Use in Space Reactor Core Heat Pipes. Tech. rep. LA-8685-MS. Los Alamos National Laboratory, 1981 (cit. on p. 28).

[137] B.L. Cox and F. Wiffen. "The ductility in bending Molybdenum Alloys Irradiated between $425 \mathrm{C}$ and 1000 C". In: Journal of Nuclear Materials 85 (1979), pp. $901-905$ (cit. on p. 28).

[138] C.L. Snead et al. Voids and other neutron-produced microstructure in Mo and Mo-0.5 at.\% Ti as studied by positron-annihilation techniques. Tech. rep. BNL 28868. Brookhaven National Laboratory (cit. on p. 28).

[139] B.V. Cockeram, R.W Smith, and L.L. Snead. "The influence of fast neutron irradiation and irradiation temperature on the tensile properties of wrought LCAC and TZM molybdenum". In: Journal of Nuclear Materials 346 (2005), pp. 145-164 (cit. on p. 28).

[140] M. Li et al. "Deformation and fracture properties in neutron irradiated pure Mo and Mo alloys". In: Journal of Nuclear Materials 376 (2008), pp. 240246 (cit. on p. 28).

[141] "Postirradiation tensile properties of Mo and Mo alloys irradiated with 600 MeV protons". In: Journal of Nuclear Materials 212 (1994), pp. 1283 -1287 (cit. on p. 28).

[142] N. Simos, H. Ludewig, and N. Catalan-Lasheras. "Thermo-mechanical Response of the Halo Intercepts with the SNS Proton Beam". In: ed. by Particle Accelerator Conference. Chicago, US, 2001 (cit. on p. 30).

[143] W. Stein et al. Thermal Stress Analyses for the NLC Positron Target. Tech. rep. SLAC-LCC-0088. Stanford: SLAC, 2002 (cit. on p. 30).

[144] O. Caretta et al. "Response of a tungsten powder target to an incident high energy proton beam". In: Phys. Rev. ST. Accel. Beams 17 (2014), p. 101005 (cit. on p. 30).

[145] A. Ahmad et al. "Generic study on the design and operation of high power targets". In: Phys. Rev. ST. Accel. Beams 17 (2014), p. 024701 (cit. on p. 30). 
[146] A. Dallocchio. "Study of Thermo-mechanical effects induced in Solids by High Energy Particle Beams: Analytical and Numerical Methods". PhD thesis. Torino: Politecnico di Torino, 2008 (cit. on pp. 30, 41, 157).

[147] M.A. Meyers. Dynamic Behavior of Materials. John Wiley \& Sons, 1994 (cit. on pp. 33, 34, 49, 63, 69).

[148] J. Zukas. Introduction to Hydrocodes. Elsevier Science, 2004 (cit. on pp. 34, $36,38,51,52,57)$.

[149] J.R. Airey. "The vibration of cylinders and cylindrical shells". In: Arch. d. Math. u. Phys. 20.3 (1913), pp. 289 -294 (cit. on p. 40).

[150] S.Y. Lin. "Coupled vibration and natural frequency analysis of isotropic cylinders or disks of finite dimensions". In: Journal of Sound and Vibration 185.2 (1995), pp. 193 -199. ISSN: 0022-460X. DOI: https://doi.org/10. 1006/jsvi.1995.0374 (cit. on pp. 40,41).

[151] H. Richter. "Simulating Transient Effects of Pulsed Beams on Beam Intercepting Devices". PhD thesis. Viena: Technischen Universitï $\frac{1}{2} \mathrm{t}$ Wien, 2011 (cit. on pp. 41, 53).

[152] R.D. Blevins. Formulas for natural frequency and mode shape. Van Nostreand Reinhold, 1979. DOI: 0-442-20710-7 (cit. on p. 43).

[153] N. Metropolis. "The beginning of the Monte Carlo Method". In: Los Alamos Science Special Issue (1987) (cit. on p. 44).

[154] Giuseppe Battistoni et al. "Overview of the FLUKA code". In: Annals of Nuclear Energy 82 (2015), pp. 10 -18. ISSN: 0306-4549. DOI: http://dx . doi.org/10.1016/j.anucene.2014.11.007 (cit. on p. 45).

[155] ANSYS Mechanical User's Guide. ANSYS Inc. 2013 (cit. on pp. 46, 50).

[156] ANSYS Mechanical APDL Command Reference. ANSYS Inc. 2013 (cit. on p. 46).

[157] MPDB software. JAHM Software, Inc. (Cit. on p. 47).

[158] P. Jacob and L. Goulding. An Explicit Finite Element Primer. NAFEMS, 2002. ISBN: 9781874376453 (cit. on pp. 49, 50).

[159] ANSYS Autodyn User Manual, Release 14.5. 2012 (cit. on pp. 51, 63, 64). 
[160] J. Von Neumann and R.D. Richtmyer. "A Method for the Numerical Calculation of Hydrodynamic Shocks". In: Journal of Applied Physics 21 (1950), p. 232 (cit. on p. 52).

[161] T.W. Eaton. Hydrodinamic Calculations on Solid and Liquid Targets. Tech. rep. Geneva, Switzerland: PS/AA/Note 84-9, CERN, 1984 (cit. on p. 52).

[162] D.C. Wilson et al. In: Hydrodynamic Calculations of 20-TeV Beam Interactions with the SSC Beam Dump. Washington DC, US: Particle Accelerator Conference (PAC 93), IEEE, 1993 (cit. on p. 52).

[163] Century Dynamics Ltd for CERN. AUTODYN Study of the Structural Behaviour of the Main LHC Absorber Block in the Case of a Total Beam Dilution Failure. Tech. rep. Horsham, UK: Century Dynamics, 2001 (cit. on p. 52).

[164] H. Richter et al. "Simulation of target response due to uranium ion beam impact". In: The European Physical Journal A 42 (2009), pp. 301-306 (cit. on pp. 53,64).

[165] A. Bertarelli et al. "Nuclear Instruments and Methods in Physics Research B". In: The European Physical Journal A b308 (2013), pp. 88-89 (cit. on pp. 53, 61).

[166] A. Bertarelli et al. In: Behaviour of advanced materials impacted by high energy particle beams. Journal of Physics: Conference Series 451012005 , 2013 (cit. on pp. 53, 64).

[167] M. Scapin, L. Peroni, and A. Dallocchio. "Effects induced by LHC high energy beam in copper structures". In: J. Nucl. Mater 420 (2012), p. 463 (cit. on p. 53).

[168] M. Scapin et al. "Numerical Simulations of Tungsten Targets hit by LHC Proton beam". In: ed. by IV International Conference on Computational Methods for Coupled Problems in Science and Engineering. Kos Island, Greece (cit. on p. 53).

[169] M. Scapin. "Shock-wave and high strain-rate phenomena in matter: modeling and applications". PhD thesis. Torino: Politecnico di Torino, 2013 (cit. on p. 53).

[170] M. Scapin, L. Peroni, and A. Dallochio. "Numerical modeling of materials under extreme conditions". In: ed. by Nicola Bonora. Springer, 2014. 
Chap. Thermo-mechanical modelling of high energy particle impacts, pp. 87106 (cit. on p. 53).

[171] N.A. Tahir et al. A Design of a Target for Antiproton Production at the Future FAIR Facility. Tech. rep. FAIR-ACCELERATORS-08. Darmstadt, Germany: FAIR, 2008 (cit. on p. 53).

[172] N.A. Tahir et al. Design of Antiproton Target for FAIR. Tech. rep. FAIRACCELERATORS-12. Darmstadt, Germany: FAIR, 2012 (cit. on p. 53).

[173] N.A. Tahir et al. "Impact of high energy high intensity proton beams on targets: Case studies for Super Proton Synchrotron and Large Hadron Collider". In: Phys. Rev. ST. Accel. Beams 15 (2012), p. 051003 (cit. on p. 53).

[174] S. Eliezer, A. Ghatak, and H. Hora. Fundamentals of Equations of State. Singapore: Word Scientific Pub Co Inc ISBN 9810248334, 2012 (cit. on p. 55).

[175] L. Avrami P. Harris. Some Physics of the Grüneisen Parameter. Tech. rep. 4423. Picatinny Arsenal, 1972 (cit. on p. 56).

[176] D. J. Steinberg. Equation of State and Strength Properties of Selected Materials. Lawrence Livermore National Laboratory, 1996 (cit. on pp. 58, 64, $69,70)$.

[177] H.J. Tillotson. Metallic Equations of State for Hypervelocity Impact. Tech. rep. US: Airforce Special Weapon Center, 1962 (cit. on p. 58).

[178] Brodie and Hormuth. The PUFF-66 Computer Program. Tech. rep. AFWLTR-66-48. Albuquerque: Air Force Weapons Laboratory, 1968 (cit. on p. 59).

[179] G.I. Kerley and T.L. Christian Frear. Sandia Equation of State Database: sesian file. Tech. rep. Albuquerque, US: United State department of Energy, 1993 (cit. on p. 59).

[180] S.P. Lyon and J.D. Johnson. SESAME: The Los Alamos National Labortory Equation of State Database. Tech. rep. LA-UR-92-3407. LANL (cit. on p. 59).

[181] G.R. Johnson and W.A Cook. "A constitutive model and data for metals subjected to large strains, high strain rates and high temperatures". In: ed. by Seventh international symposium on ballistics. The Hague, Netherlands, 1983 (cit. on p. 60). 
[182] B. Hopkinson. "A Method of Measuring the Pressure Produced in the Detonation of High Explosives or by the Impact of Bullets". In: Philos. Trans. R. Soc. London 213 (1914), p. 437 (cit. on p. 60).

[183] J.H. Hollomon. "Tensile Deformation". In: Trans. AIME 162 (1945), pp. 268290 (cit. on p. 60).

[184] F.J. Zerilli and R.W. Armstrong. "Dislocation-mechanics-based constitutive relations for material dynamics calculations". In: Journal of Applied Physics 61 (1987), p. 1816 (cit. on p. 61).

[185] A.S. Khan R. Liang. "A critical review of experimental results and constitutive models for $\mathrm{BCC}$ and $\mathrm{FCC}$ metals over a wide range of strain rates and temperatures". In: International Journal of Plasticity 15.9 (1999), pp. 963980 (cit. on p. 61).

[186] D.J. Steinberg, S.G. Cochran, and M.W. Guinan. "A constitutive model for metals applicable at highstrain rate". In: Journal of Applied Physics 51 (1980), p. 1498 (cit. on p. 62).

[187] D.J Steinberg and C.M. Lund. "A constitutive model for strain rates from 10e-4 to 10e6 s-1". In: Journal of Applied Physics 65 (1989), pp. 1528-1533 (cit. on p. 62).

[188] D.L. Preston, D.L. Tonks, and D.C. Wallace. "Model of plastic deformation for extreme loading conditions". In: Journal of Applied Physics 93.1 (2003), p. 211 (cit. on p. 62).

[189] P.S. Follansbee and U.F. Kocks. "A constitutive description of the deformation of copper based on the use of the mechanical threshold stress as an internal state variable". In: Acta Metallurgica 36 (1988), pp. 81-93 (cit. on p. 62).

[190] T. Wierzbicki and Y. Bai Y. Bao Y-W Lee. "Calibration and evaluation of seven fracture models". In: International Journal of Mechanical Sciences 47 (2005), pp. 719-743 (cit. on pp. 63, 64).

[191] D.E. Grady. "Local inertial effects in dynamic fragmentation". In: Journal of Applied Physics 53 (1982), p. 322 (cit. on p. 64).

[192] D.E. Grady. "The spall strenght of condenser matter". In: J. Mech. Phys. Solids 36.3 (1988), pp. 353-384 (cit. on p. 64). 
[193] G.R. Johnson and W.H. Cook. "Fracture characteristics of three metals subjected to various strains, strain rates, temperatures and pressures". In: Engineering Fracture Mechanics 21.1 (1985), pp. 31-48 (cit. on p. 65).

[194] A. Ferrari et al. FLUKA: A Multi-Particle Transport Code. Tech. rep. Geneva, Switzerland: CERN, 2005 (cit. on p. 66).

[195] DYNLab: Impact and high strain-rate testing laboratory (cit. on pp. 68, 72).

[196] L. Hu, P. Miller, and J. Wang. "High strain-rate spallation and fracture of tugnsten by laser-induced stress waves". In: Material Science and Engineering $A$ b308 (2013), pp. 88-99 (cit. on pp. 70, 71, 87, 98).

[197] L. Peroni et al. "Investigation of Dynamic Fracture Behavior of Graphite". In: Key Engineering Materials 569-570 (2013), pp. 103-110 (cit. on pp. 70, $71,92)$.

[198] M. Scapin et al. "Tensile Behavior of T91 Steel Over a Wide Range of Temperatures and Strain-Rate up to $10 \mathrm{e} 4$ s-1". In: Journal of Materials Engineering and Performance 23.8 (2014), pp. 3007-3017 (cit. on p. 73).

[199] J. Baird. Explosive Shocks and Impedance Mismatch in Armatures. Tech. rep. University of Missouri-Rolla, US: Rock Mechanics and Explosives Research Center, 2003 (cit. on p. 75).

[200] M.A. Cook. The Science of Industrial Explosives. Tech. rep. Salt Lake City, Utah, US: IRECO Chemicals, 1974 (cit. on p. 75).

[201] J.H. Kim et al. "Evaluation of thermal shock strengths for graphite materials using a laser irradiation method". In: Materials Science and Engineering A 387 (2004), p. 385 (cit. on p. 92).

[202] W.L. Greenstreet. Mechanical Properties of Artificial Graphite A survey report. Tech. rep. ORNL-4327. Oak Ridge National Laboratory, 1968 (cit. on p. 92).

[203] Entegris. Properties and characteristics of graphite. Tech. rep. Entegris, 2013 (cit. on p. 92).

[204] F. Stefanini and K. Kershaw. Design for handling and maintenance in radiation areas at CERN - Introduction. Tech. rep. Internal Engineering Note EDMS no. 1518744. CERN, 2015 (cit. on p. 103). 
[205] T. Bogey and R. Jones. "The beam position system of the CERN Neutrino to Grand Sasso proton beam line". In: Proceedings of DIPAC. Venice, Italy, 2007 (cit. on p. 113).

[206] E. Bravin et al. "A new TV beam observation system for CERN". In: Proceedings of DIPAC. 2005 (cit. on p. 113).

[207] SCHOTT AG. TIE-42 Radiation Resistant Optical Glasses. Technical Report. 2007 (cit. on p. 113).

[208] LED PowerStars - OSLON. ILS Intelligent Led Solutions (cit. on p. 116).

[209] Y. Yuri, T. Ishizaka, and T. Yuyama. "Use of Gafchromic Films to Measure the Transverse Intensity Distribution of a Large-area Ion Beam". In: ed. by IBIC. Tsukuba, Japan, 2012 (cit. on p. 119).

[210] CID8825D Color Radiation Hardened Camera (cit. on p. 120).

[211] Full HD WDR Day/Night Fixed Network Camera DCS-3716. D-Link (cit. on p. 120).

[212] Goodfellow Ltd. (Cit. on p. 130).

[213] A. Perez Fontenla. Quality study of tungsten (W) rods and tantalum (Ta)cladded $W$ material. Tech. rep. EDMS no: 1566868. CERN, 2015 (cit. on pp. 131, 135, 136).

[214] A. Obererlacher. Product Specication TaM rod. Tech. rep. PS-MPR-112. Plansee, 2014 (cit. on p. 132).

[215] Y. Ma et al. "An Experiment Using Neutron Diffraction to Investigate Residual Strain Distribution in a Hot Isostatic Pressed (HIPPED) Target Plate". In: Materials Today: Proceedings 2 (2015), S267-S273 (cit. on p. 134).

[216] C. Bungau et al. "Power upgrade studies for the ISIS-TS1 spallation target". In: Proceedings of IPAC2014 THPRI082 (2014) (cit. on p. 134).

[217] A. Perez Fontenla. Microscope observation of tantalum coating on tungsten rods. Tech. rep. EDMS no: 1513398. CERN, 2015 (cit. on p. 134). 
[218] A. Perez Fontenla. Scanning electron microscope (SEM) observation of tantalum (Ta) sleeved tungsten $(W)$ rod. Tech. rep. EDMS no: 1538148. CERN, 2015 (cit. on p. 134).

[219] A. Celzard, S. Schneider, and J.F. Mareche. "Densification of expanded graphite". In: Carbon 40.12 (2002), pp. 2185 -2191. ISSN: 0008-6223 (cit. on p. 211).

[220] D.D.L. Chung. "Exfoliation of graphite". In: Journal of Materials Science 22.12 (1987), pp. 4190-4198. ISSN: 1573-4803 (cit. on p. 211).

[221] F.R. Menter, M. Kuntz, and R. Langtry. "Experience with the SST Turbulence Model". In: Turbulence, Heat and Mass Transfer 4 (2003), pp. 625 -632 (cit. on p. 222).

[222] B. Majidi. "Fatigue Life and Short Crack Behavior in Ti-6Al-4V Alloy Interactions of Foreign Object Damage, Stress, and Temperature". In: Met. and Mater. Trans. A 39A (2008), p. 772 (cit. on pp. 230, 231).

[223] S.J. Maddox. Fatigue Strength of Welded Structures. Woodhead Publishing Series in Welding and Other Joining Technologies. Elsevier Science, 2014 (cit. on p. 230).

[224] J. Goodman. Mechanics Applied to Engineering. Longsmans-Green, 1899 (cit. on p. 231).

[225] F.C. Campbell. Elements of Metallurgy and Engineering Alloys. ASM International, 2008. ISBN: 9781615030583 (cit. on p. 231).

[226] A.G. Palmgren. "Die Lebensdauer von Kugellagern". In: Zeitschrift des Vereines Deutscher Ingenieure 68.14 (1924), pp. 339-341 (cit. on p. 232).

[227] P. Marmy and T. Leguey. "Impact of irradiation on the tensile and fatigue properties of two titanium alloys". In: Journal of Nuclear Materials 296.1-3 (2001). 4th Int. Workshop on Spallation Materials Technology, pp. $155-$ 164. ISSN: 0022-3115. DOI: http://dx.doi.org/10.1016/S0022-3115(01) 00564-5 (cit. on p. 232).

[228] RaDIATE Collaboration (cit. on p. 235). 